\title{
Direct Use of Carboxylic Acids in the Photocatalytic Hydroacylation of Styrenes To Generate Dialkyl Ketones
}

\author{
Jesus I. Martinez Alvarado, Alyssa B. Ertel, Andrea Stegner, Erin E. Stache, Abigail G. Doyle* \\ Department of Chemistry, Frick Chemistry Laboratory, Princeton University, Princeton, New \\ Jersey, 08544, USA. \\ Corresponding author email: agdoyle@princeton.edu \\ Supporting Information
}




\section{Table of Contents}

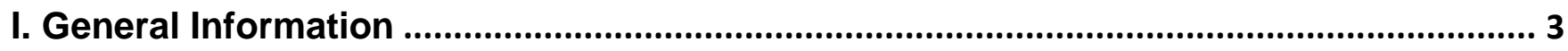

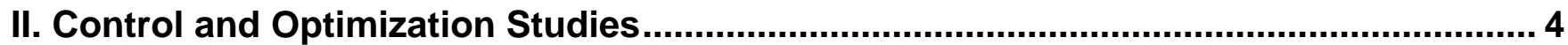

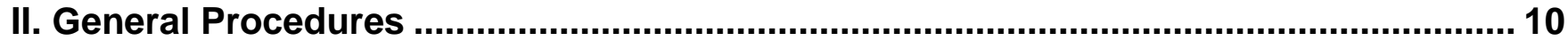

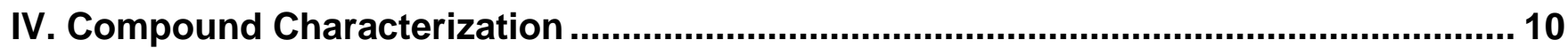

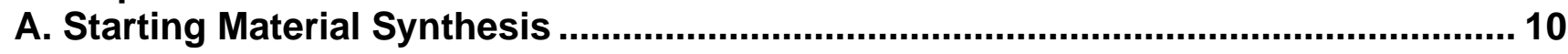

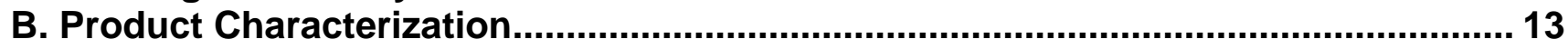

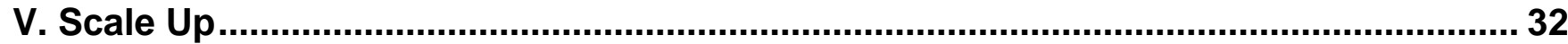

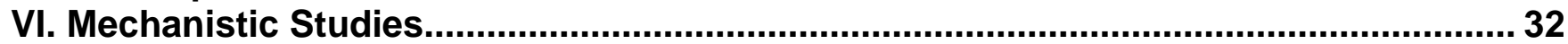

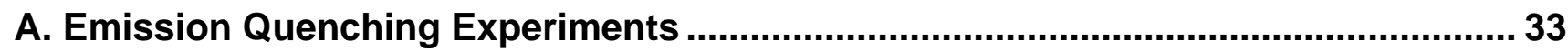

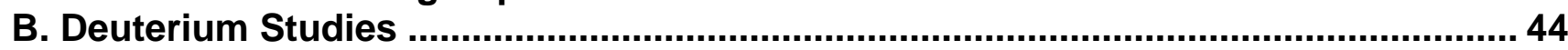

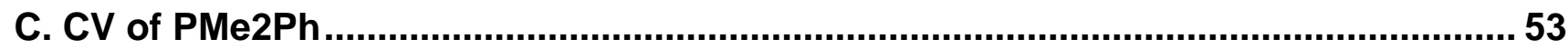

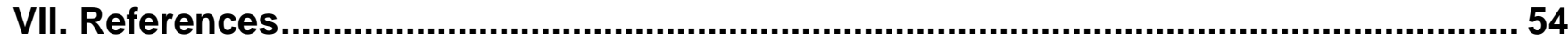




\section{General Information}

Materials. Commercial reagents were acquired from Sigma-Aldrich, Alfa Aesar, Acros, Strem, $\mathrm{TCl}$, or Oakwood and used as received. Diethyl ether ( $\left.\mathrm{Et}_{2} \mathrm{O}\right)$, tetrahydrofuran (THF), and toluene $(\mathrm{PhMe})$ were dried by passing through activated alumina columns and stored over molecular sieves in a $\mathrm{N}_{2}$-filled glovebox; $\mathrm{N}, \mathrm{N}$-dimethylformamide (DMF) was dried by passing through a column of activated molecular sieves. Acetonitrile (MeCN) was purchased from Millipore Sigma without sieves and subsequently sparged with nitrogen before bringing it into the glovebox. Sieves were detrimental for reactivity.

Instrumentation. Proton nuclear magnetic resonance $\left({ }^{1} \mathrm{H}\right.$ NMR) spectra were recorded on a Bruker 500 AVANCE spectrometer ( $500 \mathrm{MHz})$, a Bruker NB 300 spectrometer $(300 \mathrm{MHz})$, or a Bruker Avance III HD NanoBay (400 MHz) spectrometer. Deuterium nuclear magnetic resonance $\left({ }^{2} \mathrm{H}\right.$ NMR) spectra were recorded on a Bruker 500 AVANCE spectrometer $(77 \mathrm{MHz})$. Carbon nuclear magnetic resonance $\left({ }^{13} \mathrm{C}\right.$ NMR) spectra were recorded on a Bruker 500 AVANCE spectrometer $(126 \mathrm{MHz})$. Fluorine nuclear magnetic resonance ( $\left.{ }^{19} \mathrm{~F} \mathrm{NMR}\right)$ spectra were recorded on a Bruker NB 300 spectrometer $(282 \mathrm{MHz})$. Chemical shifts for protons are reported in parts per million $(\mathrm{ppm})$ downfield from tetramethylsilane and are referenced to residual protium in the NMR solvent $\left(\mathrm{CHCl}_{3}=\delta 7.26 \mathrm{ppm}\right)$. Chemical shifts for carbon are reported in parts per million downfield from tetramethylsilane and are referenced to the carbon resonances of the solvent residual peak $\left(\mathrm{CDCl}_{3}=\delta 77.16 \mathrm{ppm}\right)$. Chemical shifts for fluorine are reported in parts per million referenced to $\mathrm{CFCl}_{3}(\delta 0 \mathrm{ppm})$. NMR data are represented as follows: chemical shift $(\delta \mathrm{ppm})$, multiplicity $(\mathrm{s}=$ singlet, $\mathrm{bs}=$ broad singlet, $\mathrm{d}=$ doublet, appd $=$ apparent doublet, $\mathrm{t}=$ triplet, $q=$ quartet, $p=$ pentet, $s x=$ sextet $m=$ multiplet), coupling constant in Hertz $(\mathrm{Hz})$, integration. Reversed-phase liquid chromatography/mass spectrometry (LC/MS) was performed on an Agilent 1260 Infinity analytical LC and Agilent 6120 Quadrupole LC/MS system, using electrospray ionization/atmospheric-pressure chemical ionization (ESI/APCI), and UV detection at 254 and $280 \mathrm{~nm}$. High-resolution mass spectra were obtained on an Agilent 6220 LC/MS using electrospray ionization time-of-flight (ESI-TOF) or Agilent 7200 gas chromatography/mass spectrometry using electron impact time-of-flight (EI-TOF). Gas chromatography was performed on an Agilent $7890 \mathrm{~A}$ series instrument equipped with a split-mode capillary injection system and flame ionization detectors. Fourier transform infrared (FT-IR) spectra were recorded on a PerkinElmer Spectrum 100 and are reported in terms of frequency of absorption $\left(\mathrm{cm}^{-1}\right)$. High-performance liquid chromatography (HPLC) was performed on an Agilent 1200 series instrument with a binary pump and a diode array detector, using Chiralcel OD-H $(25 \mathrm{~cm} \times 0.46 \mathrm{~cm})$, Chiralcel OJ-H $(25 \mathrm{~cm} \times 0.46 \mathrm{~cm})$, Chiralpak AS-H $(25 \mathrm{~cm} \times 0.46 \mathrm{~cm})$, Chiralpak AD-H $(25 \mathrm{~cm} \times 0.46 \mathrm{~cm})$, Chiralpak IC $(25 \mathrm{~cm} \times 0.46 \mathrm{~cm})$ and Chiralpack ID $(25 \mathrm{~cm} \times 0.46 \mathrm{~cm})$.

Light Sources. Reactions were initially optimized on 34 W blue LED lamps (KSH150B Grow Light Blue) purchased from Kessil. When Kessil lamps were used, they were placed $2 \mathrm{~cm}$ away from 1-dram reaction vials without the use of fans. Isolations yields were obtained using a Photoreactor (PR). Photoreactors were generously obtained from the MacMillan lab. A Penn OC Photoreactor M1 series was used with a $450 \mathrm{~nm}$ light source. ${ }^{1}$ 


\section{Control and Optimization Studies}

Procedure for reaction optimization: An oven-dried 1-dram reaction vial (VWR® glass vials, 66011-041) was charged with carboxylic acid ( $0.1 \mathrm{mmol}, 1.0$ equiv) and equipped with a PTFE-coated stir bar (VWR® Micro stir bars, $2 \times 7 \mathrm{~mm}, 58948-976)$. The vial was Teflon taped on the threads, and then taken into a $\mathrm{N}_{2}$-filled glovebox. To the vial was added $\mathrm{MeCN}(0.1 \mathrm{M})$, alkene $(0.3 \mathrm{mmol}, 3.0$ equiv) and base $(0.1 \mathrm{mmol}, 1.0$ equiv). From a stock solution was added [Ir(dF-Me-ppy) 2 dtbbpy]PF 6 (2 mg, $0.002 \mathrm{mmol}, 0.02$ equiv) and $\mathrm{Ph}_{2} \mathrm{~S}_{2}(1.0 \mathrm{mg}, 0.005 \mathrm{mmol}$, 0.05 equiv). Finally, phosphine $(0.1 \mathrm{mmol}, 1.0$ equiv) was added. The vial was then capped and sealed with electrical tape. The vial was irradiated for $24 \mathrm{~h}$ with $34 \mathrm{~W}$ blue LEDs. An aliquot of the crude reaction mixture was analzed by ${ }^{1} \mathrm{H}$-NMR with 1 -fluoronaphthalene $(0.1$ mmol, 1.0 equiv) as an external standard.

Our initial efforts began employing 3-(4-fluorophenyl)propanoic acid with 1-(trifluoromethyl)-2vinylbenzene as alkene acceptor and ethyl diphenyl phosphinite as mediator.

Table S1. Control and optimization of the coupling reaction between 3-(4-fluorophenyl)propanoic acid and 1-(trifluoromethyl)-2-vinylbenzene

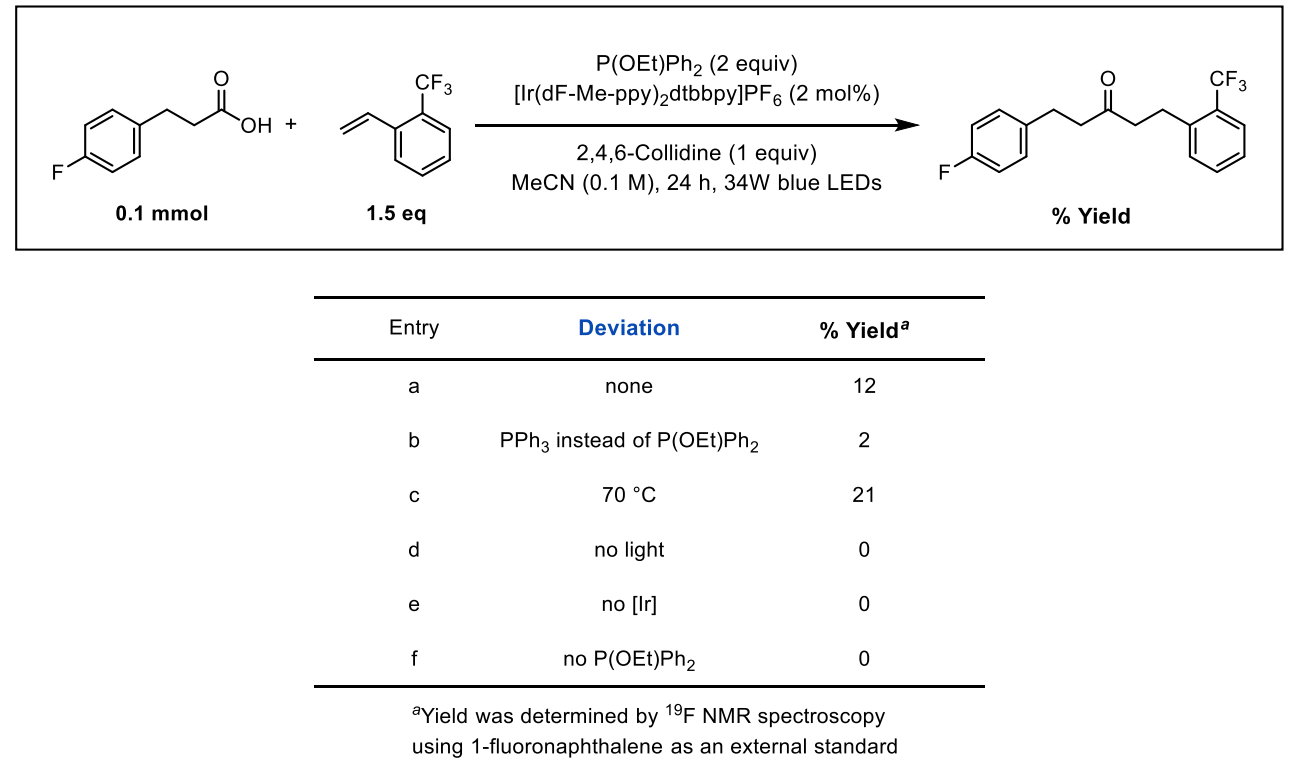

Table S2. Optimization of solvent using ethyl diphenyl phosphinite as phosphine mediator.

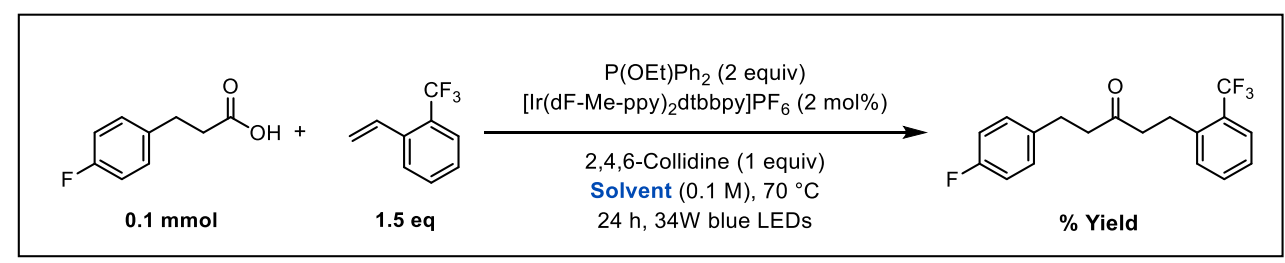




\begin{tabular}{ccc}
\hline Entry & Solvent & ${\text { \% } \text { Yield }^{a}}^{\text {a }}$ \\
\hline a & TFT & 5 \\
b & MTBE & 0 \\
c & DME & 3 \\
d & NMP & 4 \\
e & Et ${ }_{2} \mathrm{O}$ & 0 \\
f & THF & 0 \\
g & DMF & 6 \\
h & DMA & 12 \\
i & PhOMe & 8 \\
j & MeCN & 19 \\
\hline
\end{tabular}

${ }^{a}$ Yield was determined by ${ }^{19} \mathrm{~F}$ NMR spectroscopy

using 1-fluoronaphthalene as an external standard

Table S3. Optimization of bases using ethyl diphenyl phosphinite as phosphine mediator.

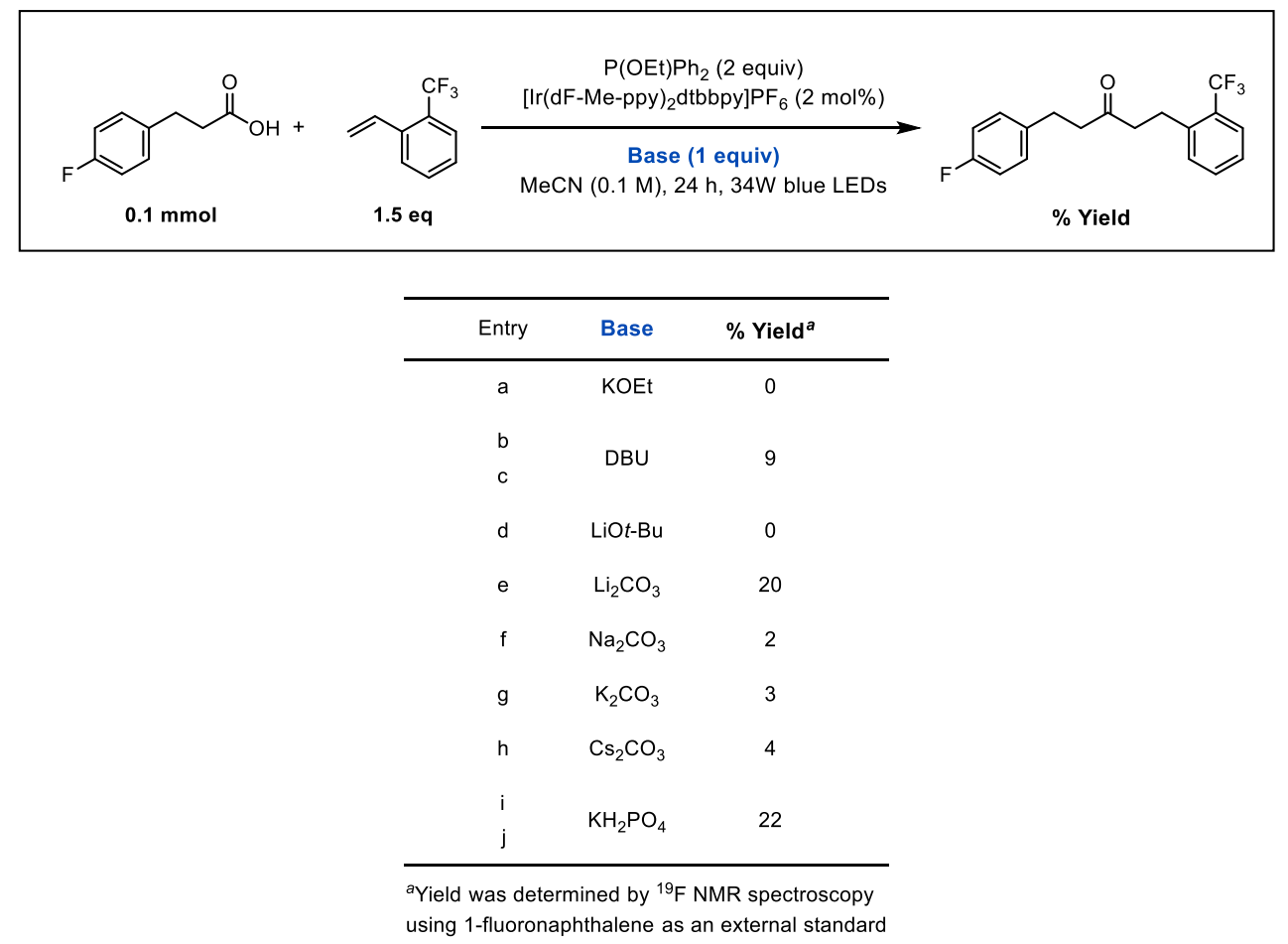

Given the poor success of ethyl diphenyl phosphinite in this reaction, emission quenching experiments were undertaken (See Section V). More importantly, other phosphines were explored for this reaction. The alkene acceptor was substituted for $\alpha$-methyl styrene in order to bias the reaction toward radical addition. 


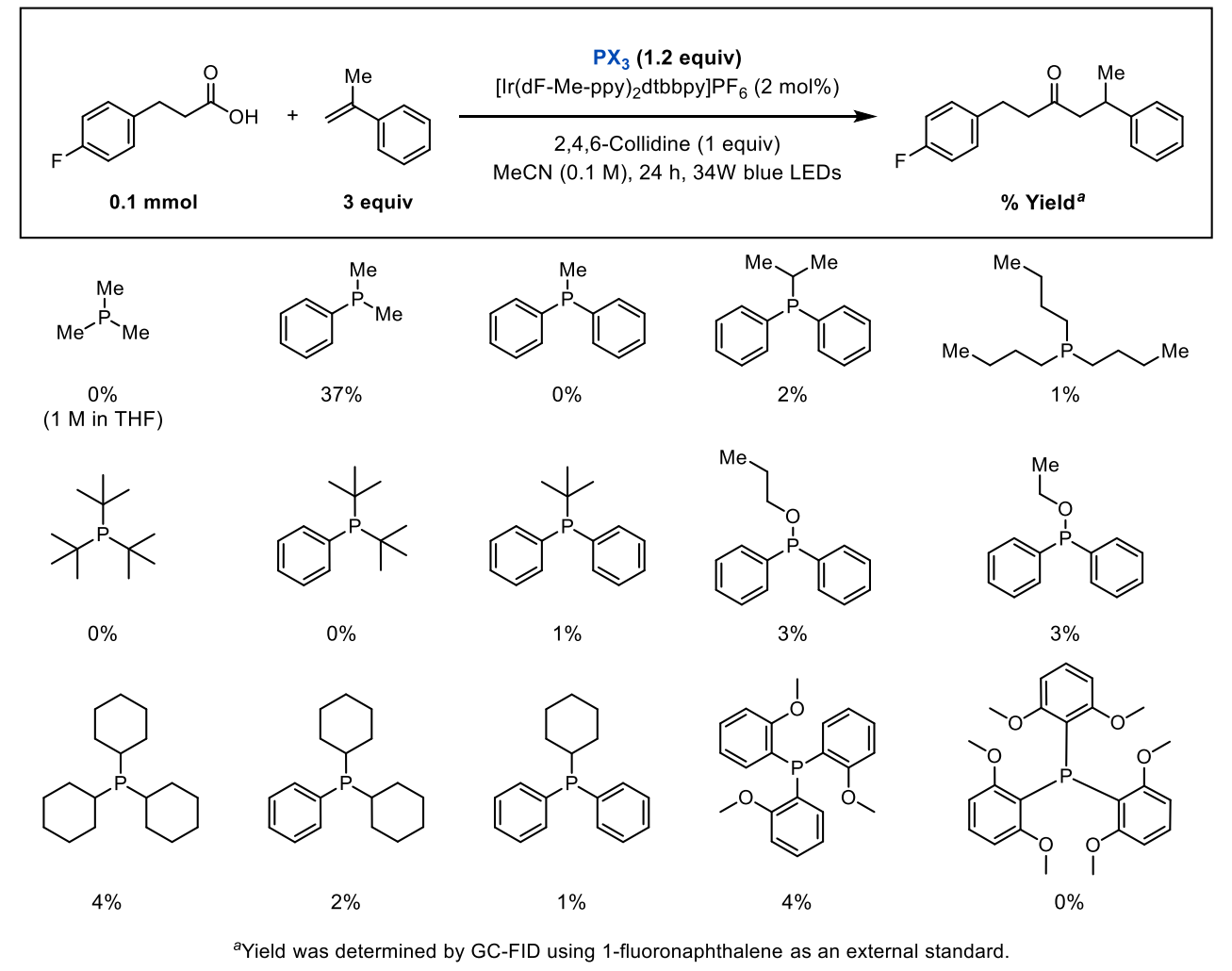

Figure S1. Optimization of phosphines using 3-(4-fluorophenyl)propanoic acid and alpha methyl styrene.

Table S4. Examination of photocatalysts.

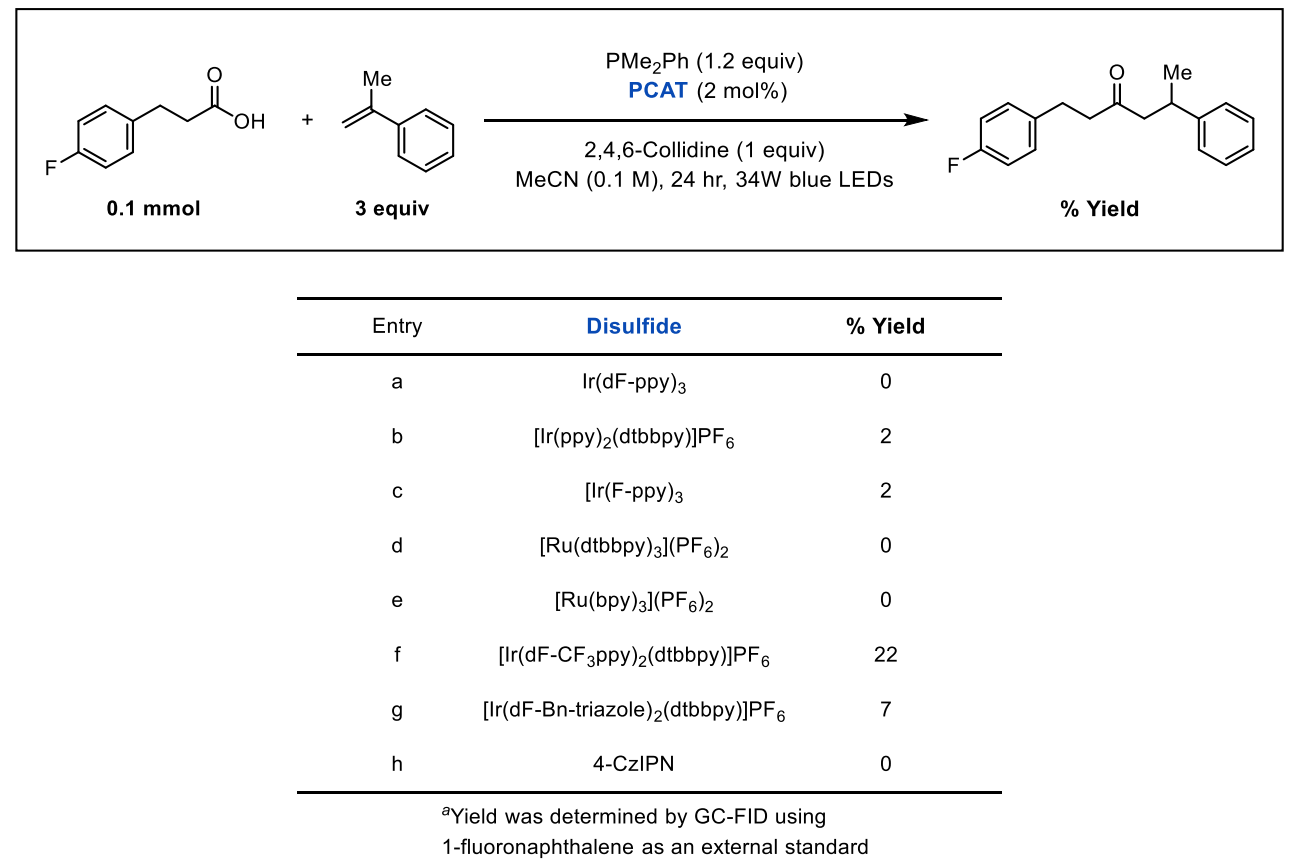

Table S5. Examination of hydrogen atom donors. 


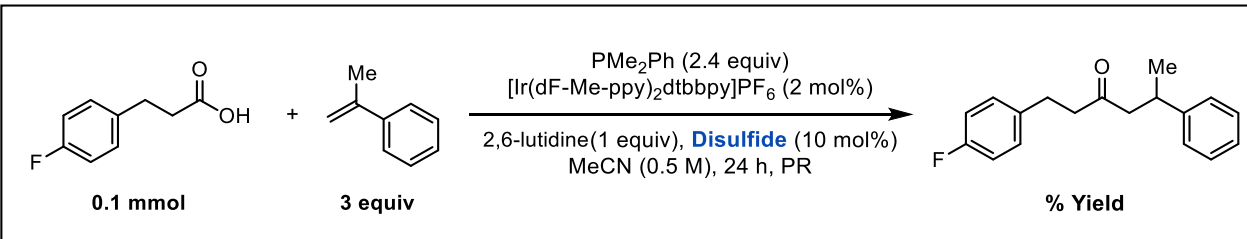

\begin{tabular}{ccc}
\hline Entry & Disulfide & \% Yield \\
\hline a & none & 44 \\
b & $\mathrm{tBu}_{2} \mathrm{~S}_{2}$ & 41 \\
c & $\mathrm{iPr}_{2} \mathrm{~S}_{2}$ & 41 \\
d & $\mathrm{Ph}_{2} \mathrm{~S}_{2}$ & 56 \\
e & $\mathrm{TRIP}_{2} \mathrm{~S}_{2}$ & 38 \\
$\mathrm{f}$ & $(4-\mathrm{Cl}-\mathrm{Ph})_{2} \mathrm{~S}_{2}$ & 57 \\
$\mathrm{~g}$ & $(4-\mathrm{MeO}-\mathrm{Ph})_{2} \mathrm{~S}_{2}$ & 57 \\
$\mathrm{~h}$ & $\mathrm{Ph} \mathrm{CHCN}_{2}$ & 37 \\
\hline
\end{tabular}

${ }^{a}$ Yield was determined by GC-FID using

1-fluoronaphthalene as an external standard

Table S6. Examination of phenyl disulfide loading. Yield was determined by GC-FID using 1fluoronaphthalene as an external standard.

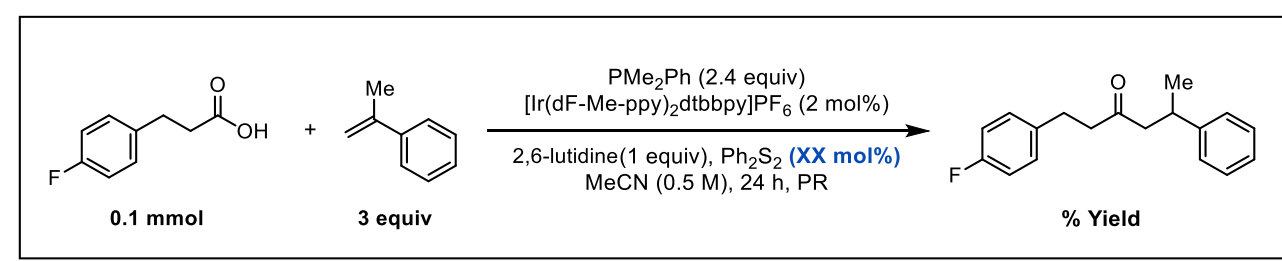

\begin{tabular}{ccc}
\hline Entry & XX mol\% & \% Yield $^{\mathbf{a}}$ \\
\hline a & 5 & 47 \\
b & 10 & 38 \\
c & 20 & 15 \\
\hline
\end{tabular}

aYield was determined by GC-FID using

1 -fluoronaphthalene as an external standard

Table S7. Examination of base additives. Yield was determined by GC-FID using 1-fluoronaphthalene as an external standard.

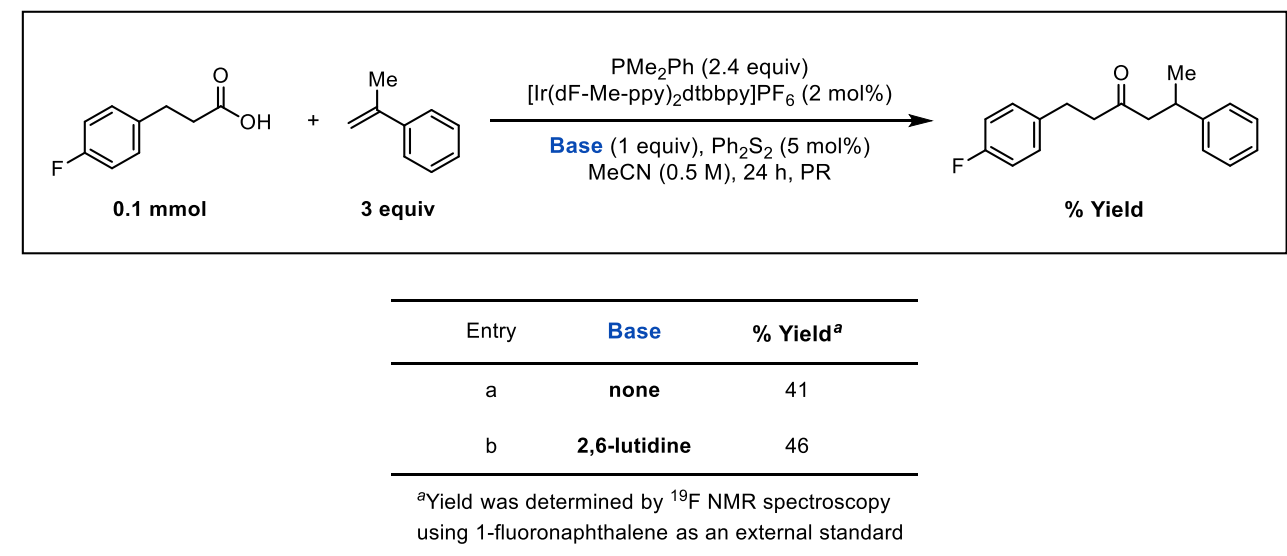


Table S8. Optimization of Phosphine loading using methyl diphenyl phosphinite, 3-(4-fluorophenyl)propanoic acid, and alpha methyl styrene.

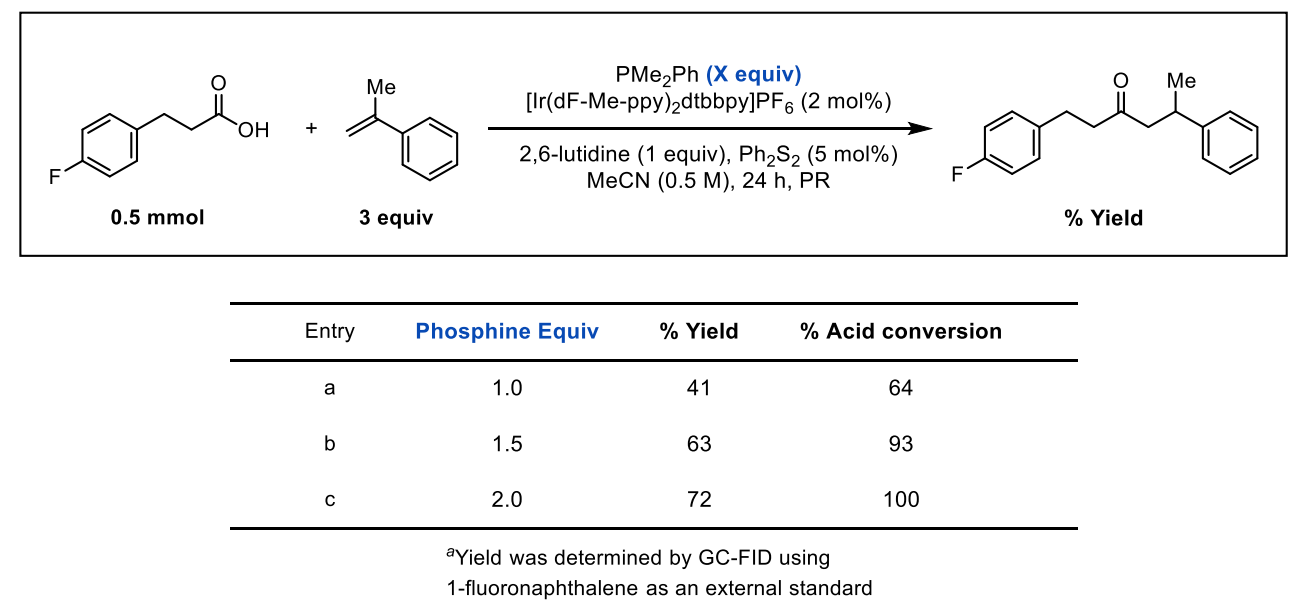

Table S9. Deoptimization table highlights the need for Iridium and phosphine for reactivity. While kessils provide moderate yield, Photoreactors were used because they provide a standardized light and temperature set up. Use of 5-cyclohexylpentanoic acid on $0.5 \mathrm{mmol}$ scale without diphenyl disulfide results in lower yield. The yield was restored by using 2.4 equivalents of phosphine. Diphenyl disulfide was removed from non-polar carboxylic acids to provide an easier isolation.

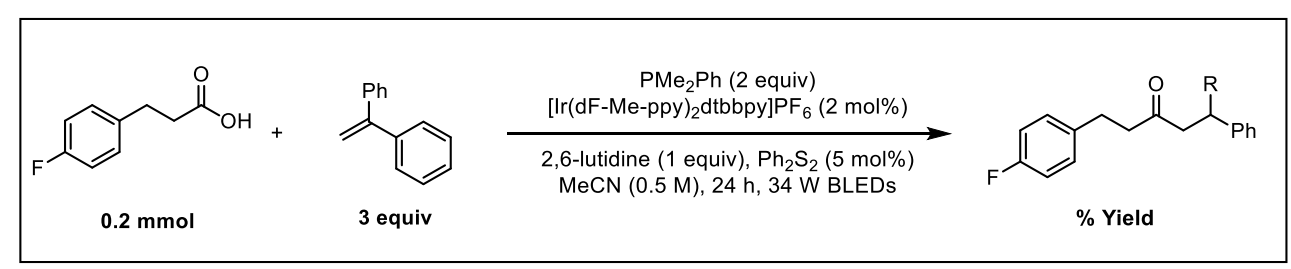




\begin{tabular}{ccc}
\hline Entry & Deviation & $\%$ Yield \\
\hline a & Merck Photoreactor & $81^{b}$ \\
b & none & 64 \\
C & no photocatalyst & 0 \\
d & no phosphine disulfide & 0 \\
e & no base & 62 \\
f & 5-cyclohexylpentanoic acid & 60 \\
g & 5-cyclohexylpentanoic acid and no $\mathrm{Ph}_{2} \mathrm{~S}_{2}$ & $92^{b}$ \\
h & 5-cyclohexylpentanoic acid, no $\mathrm{Ph}_{2} \mathrm{~S}_{2}$, and 2.4 equiv $\mathrm{PMe}_{2} \mathrm{Ph}$ & $90^{b}$ \\
\hline
\end{tabular}

a Yield was determined by ${ }^{1} \mathrm{H}$ NMR spectroscopy using 1-fluoronaphthalene as an external standard.

${ }^{b} 0.5 \mathrm{mmol}$ scale in a Photoreactor

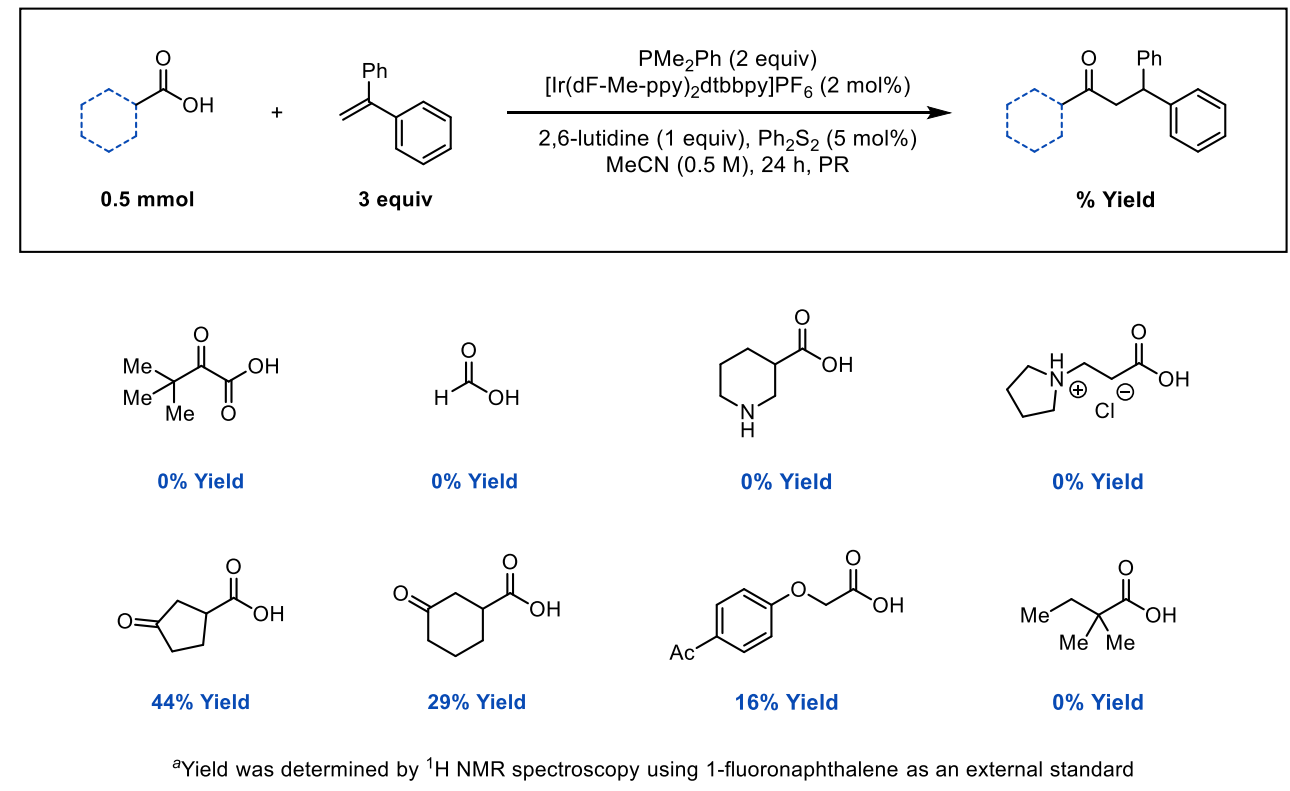

Figure S2. Poorly performing carboxylic acids when coupling with 1,1 diphenyl ethylene.

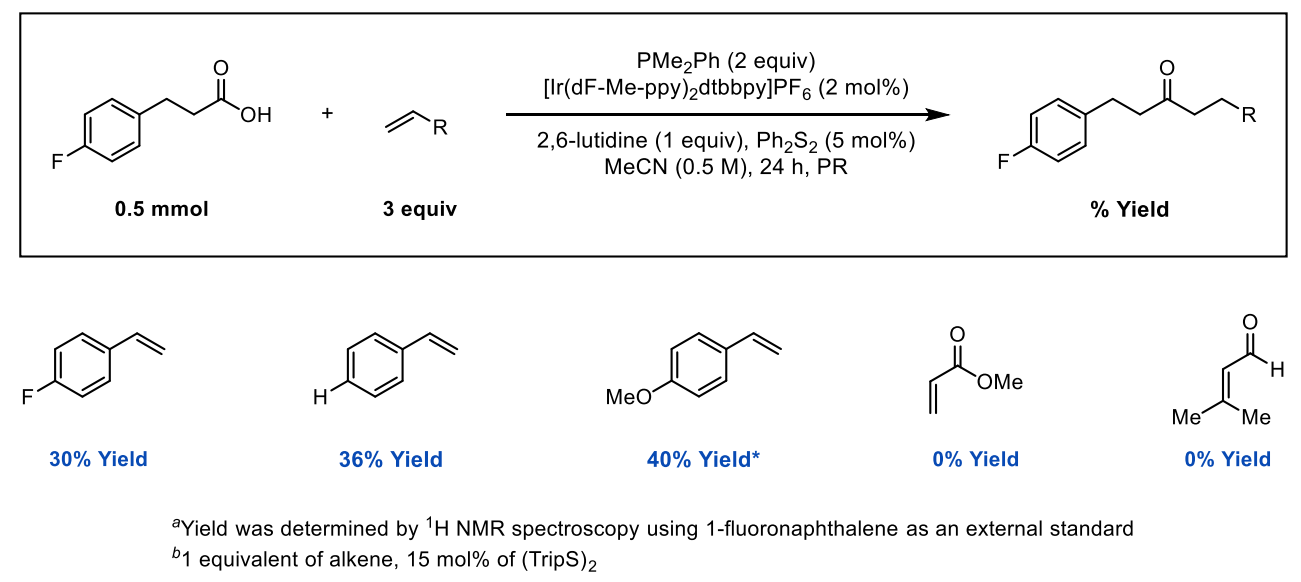

Figure S3. Poorly performing alkenes when coupling with 4-fluoro hydrocinnamic acid. MoritaBaylis Hillman reactivity was observed by GC-MS for the acrylate derivatives. 


\section{General Procedures}

General procedure A. An oven-dried 1-dram reaction vial (VWR® glass vials, 66011-041) was charged with carboxylic acid $(0.5 \mathrm{mmol}, 1.0$ equiv) and equipped with a PTFE-coated stir bar (VWR® Micro stir bars, $2 \times 7 \mathrm{~mm}, 58948-976)$. The vial was Teflon taped on the threads, and then taken into a $\mathrm{N}_{2}$-filled glovebox. To the vial was added MeCN $(0.5 \mathrm{M})$, alkene (265 $\mu \mathrm{L}$, $1.5 \mathrm{mmol}, 3.0$ equiv) and 2,6-lutidine ( $58 \mu \mathrm{L}, 0.5 \mathrm{mmol}, 1.0$ equiv). From a stock solution was added [lr] ( $8.7 \mathrm{mg}, 0.01 \mathrm{mmol}, 0.017$ equiv) and $\mathrm{Ph}_{2} \mathrm{~S}_{2}$ ( $5.46 \mathrm{mg}, 0.025 \mathrm{mmol}, 0.05$ equiv). Finally, phosphine ( $142 \mu \mathrm{L}, 1.0 \mathrm{mmol}, 2.0$ equiv) was added. The vial was then capped and sealed with electrical tape. The vial was irradiated for $24 \mathrm{~h}$ in a Photoreactor (800 rpm, 1500 fan speed, $100 \%$ light intensity). An aliquot of the crude reaction mixture was analzed by ${ }^{1} \mathrm{H}$ NMR with 1-fluoronaphthalene ( $65 \mu \mathrm{L}, 0.5 \mathrm{mmol}, 1.0$ equiv) as an external standard.

General procedure B. An oven-dried 1-dram reaction vial (VWR $\circledast$ glass vials, 66011-041) was charged with carboxylic acid $(0.5 \mathrm{mmol}, 1.0$ equiv) and equipped with a PTFE-coated stir bar (VWR® Micro stir bars, $2 \times 7 \mathrm{~mm}, 58948-976$ ). The vial was Teflon taped on the threads, and then taken into a $\mathrm{N}_{2}$-filled glovebox. To the vial was added MeCN $(0.5 \mathrm{M})$, alkene $(265 \mu \mathrm{L}$, $1.5 \mathrm{mmol}, 3.0$ equiv) and 2,6-lutidine ( $58 \mu \mathrm{L}, 0.5 \mathrm{mmol}, 1.0$ equiv). From a stock solution was added [Ir] ( $8.7 \mathrm{mg}, 0.01 \mathrm{mmol}, 0.017$ equiv). Finally, phosphine ( $171 \mu \mathrm{L}, 1.2 \mathrm{mmol}, 2.4$ equiv) was added. The vial was then capped and sealed with electrical tape. The vial was irradiated for $24 \mathrm{~h}$ in a Photoreactor (800 rpm, 1500 fan speed, 100\% light intensity). An aliquot of the crude reaction mixture was analzed by ${ }^{1} \mathrm{H}-\mathrm{NMR}$ with 1 -fluoronaphthalene $(65 \mu \mathrm{L}, 0.5 \mathrm{mmol}$, 1.0 equiv) as an external standard.

\section{Compound Characterization}

\section{A. Starting Material Synthesis}<smiles></smiles>

1-chloro-4-(1-phenylvinyl)benzene (S33) was prepared on a $23.08 \mathrm{mmol}$ scale according to a published literature procedure. ${ }^{2}$ The title compound was isolated using automated column chromatography eluting with Hexanes $(10 \mathrm{CV})$. The title compound was concentrated to produce a colorless oil ( $4.05 \mathrm{~g}, 82 \%$ yield).

${ }^{1} \mathrm{H}$ NMR $\left(500 \mathrm{MHz} \mathrm{CDCl}_{3}\right): \delta 7.41-7.17(\mathrm{~m}, 8 \mathrm{H}), 5.44(\mathrm{~d}, J=9.1 \mathrm{~Hz}, 2 \mathrm{H})$.

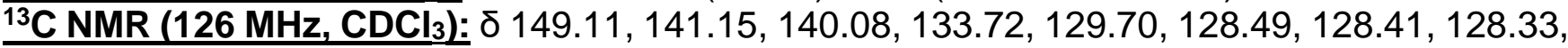
128.07, 114.85 .<smiles>C=C(C)C1=CC=CC1</smiles>

1-methyl-4-(1-phenylvinyl)benzene (S34) was prepared on a $12.33 \mathrm{mmol}$ scale according to a published literature procedure. ${ }^{2}$ The title compound was isolated using automated column 
chromatography eluting with Hexanes $(10 \mathrm{CV})$. The title compound was concentrated to produce a colorless oil $(2.24 \mathrm{~g}, 94 \%$ yield).

1H NMR (500 MHz, CDCl $)$ : $\delta 7.36(\mathrm{dt}, J=7.1,3.1 \mathrm{~Hz}, 5 \mathrm{H}), 7.30-7.25(\mathrm{~m}, 2 \mathrm{H}), 7.17(\mathrm{~d}, J=7.9$ $\mathrm{Hz}, 2 \mathrm{H}), 5.45$ (d, J=13.3 Hz, 2H), $2.40(\mathrm{~s}, 3 \mathrm{H})$.

${ }^{13} \mathrm{C} \mathrm{NMR}\left(126 \mathrm{MHz}_{1} \mathrm{CDCl}_{3}\right)$ : $\delta$ 150.03, 141.83, 138.74, 137.66, 129.00, 128.43, 128.29, 128.26, 127.77, 113.79, 21.32.<smiles>C=C(CCCCCCCCCC)c1ccccc1</smiles>

hex-1-en-2-ylbenzene (S35) was prepared on a $5.28 \mathrm{mmol}$ scale according to a published literature procedure. ${ }^{2}$ The title compound was isolated using automated column chromatography eluting with Hexanes (10 CV). The title compound was concentrated to produce a colorless oil (428 mg, 50\% yield). Spectral data were consistent with reported literature values. ${ }^{4}$

1H NMR (400 MHz, $\left.\mathbf{C D C l}_{3}\right): \delta 7.44-7.39(\mathrm{~m}, 2 \mathrm{H}), 7.37-7.30(\mathrm{~m}, 2 \mathrm{H}), 7.29-7.24(\mathrm{~m}, 1 \mathrm{H})$, $5.27(\mathrm{~d}, J=1.6 \mathrm{~Hz}, 1 \mathrm{H}), 5.06(\mathrm{~d}, J=1.5 \mathrm{~Hz}, 1 \mathrm{H}), 2.51(\mathrm{t}, J=7.5 \mathrm{~Hz}, 2 \mathrm{H}), 1.49-1.29(\mathrm{~m}, 4 \mathrm{H})$, $0.91(\mathrm{t}, J=7.2 \mathrm{~Hz}, 3 \mathrm{H})$.

${ }^{13} \mathrm{C}$ NMR (101 MHz, $\left.\mathrm{CDCl}_{3}\right): \delta 148.93,141.67,128.36,127.37,126.27,112.14,35.22,30.61$, 22.56, 14.07 .

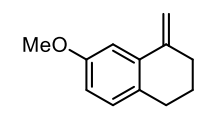

7-methoxy-1-methylene-1,2,3,4-tetrahydronaphthalene (S36) was prepared on a 11.35 mmol scale according to a published literature procedure. ${ }^{2}$ The title compound was isolated using automated column chromatography eluting with Hexanes (10 CV). The title compound was concentrated to produce a colorless oil (1.17 g, 59\% yield). Spectral data were consistent with reported literature values. ${ }^{5}$

1H NMR (500 MHz, CDCl 3 ): $\delta 7.17(\mathrm{~d}, J=2.7 \mathrm{~Hz}, 1 \mathrm{H}), 7.03(\mathrm{~d}, J=8.4 \mathrm{~Hz}, 1 \mathrm{H}), 6.78$ (dd, $J=$ 8.4, 2.7 Hz, 1H), $5.46(\mathrm{~d}, J=1.3 \mathrm{~Hz}, 1 \mathrm{H}), 4.96(\mathrm{~d}, J=1.4 \mathrm{~Hz}, 1 \mathrm{H}), 3.82(\mathrm{~s}, 3 \mathrm{H}), 2.79(\mathrm{t}, J=6.3$ $\mathrm{Hz}, 2 \mathrm{H}), 2.53$ (ddt, $J=7.7,4.2,1.4 \mathrm{~Hz}, 2 \mathrm{H}), 1.87$ (p, J = 6.3 Hz, 2H).

${ }^{13} \mathrm{C}$ NMR (126 MHz, $\left.\mathrm{CDCl}_{3}\right)$ : $\delta$ 157.86, 143.71, 135.68, 130.26, 130.00, 114.47, 108.67, 108.16, 55.46, 33.31, 29.77, 24.19.<smiles>C=C(C)c1ccc(Cl)cc1</smiles>

1-chloro-4-(prop-1-en-2-yl)benzene (S38) was prepared on a $32.3 \mathrm{mmol}$ scale according to a published literature procedure. ${ }^{2}$ The title compound was isolated using automated column chromatography eluting with Hexanes $(10 \mathrm{CV})$. The title compound was concentrated to produce a colorless oil (4.94 g, 100\% yield). Spectral data were consistent with reported literature values. ${ }^{3}$ 1H NMR (400 MHz, CDCl $\mathbf{~ M D ~}_{3}: \delta .43-7.36(\mathrm{~m}, 2 \mathrm{H}), 7.34-7.25(\mathrm{~m}, 2 \mathrm{H}), 5.36(\mathrm{~s}, 1 \mathrm{H}), 5.10(\mathrm{~h}, J$ $=1.4 \mathrm{~Hz}, 1 \mathrm{H}), 2.14(\mathrm{~d}, J=1.2 \mathrm{~Hz}, 3 \mathrm{H})$.

${ }^{13} \mathrm{C}$ NMR (101 MHz, $\left.\mathrm{CDCl}_{3}\right): \delta 142.33,139.83,133.33,128.47,126.96,113.10,21.88$.<smiles>C=C(C)c1ccc(Br)cc1</smiles>

1-bromo-4-(prop-1-en-2-yl)benzene (S39) was prepared on a $11.35 \mathrm{mmol}$ scale according to a published literature procedure. ${ }^{2}$ The title compound was isolated using automated column chromatography eluting with Hexanes (10 CV). The title compound was concentrated to produce a colorless oil (1.63 g, 72\% yield). Spectral data were consistent with reported literature values. ${ }^{3}$ 1H NMR (400 MHz, $\left.\mathrm{CDCl}_{3}\right)$ : $\delta 7.47-7.42(\mathrm{~m}, 2 \mathrm{H}), 7.35$ - $7.31(\mathrm{~m}, 2 \mathrm{H}), 5.36(\mathrm{bs}, 1 \mathrm{H}), 5.10$ (p, $J=1.5 \mathrm{~Hz}, 1 \mathrm{H}), 2.20-2.01(\mathrm{~m}, 3 \mathrm{H})$. 
${ }^{13} \mathrm{C}$ NMR $\left(101 \mathrm{MHz}, \mathrm{CDCl}_{3}\right): \delta 142.38,140.28,131.43,127.31,121.48,113.20,21.83$.<smiles></smiles>

(R)-1-(tert-butoxycarbonyl)piperidine-3-carboxylic acid (S41) was prepared on a $4.03 \mathrm{mmol}$ scale according to a published literature procedure. ${ }^{6}$ The title compound was isolated using automated column chromatography eluting with $\mathrm{MeOH}: \mathrm{DCM}(0 \% 4 \mathrm{CV}, 0-10 \% 10 \mathrm{CV}, 10 \% 6$ $\mathrm{CV})$. The title compound was concentrated to produce a white solid (655 $\mathrm{mg}, 72 \%$ yield).

1H NMR (500 MHz, ((CD $)_{2}$ SO): $\delta 12.37(\mathrm{~s}, 1 \mathrm{H}), 4.17-3.81(\mathrm{~m}, 1 \mathrm{H}), 3.69(\mathrm{~s}, 1 \mathrm{H}), 3.17-2.76$ $(\mathrm{m}, 2 \mathrm{H}), 2.31(\mathrm{~d}, J=9.1 \mathrm{~Hz}, 1 \mathrm{H}), 1.90(\mathrm{~d}, J=12.5 \mathrm{~Hz}, 1 \mathrm{H}), 1.65-1.59(\mathrm{~m}, 1 \mathrm{H}), 1.54-1.49(\mathrm{~m}$, $1 \mathrm{H}), 1.39(\mathrm{~s}, 9 \mathrm{H}), 1.38-1.28(\mathrm{~m}, 1 \mathrm{H})$.
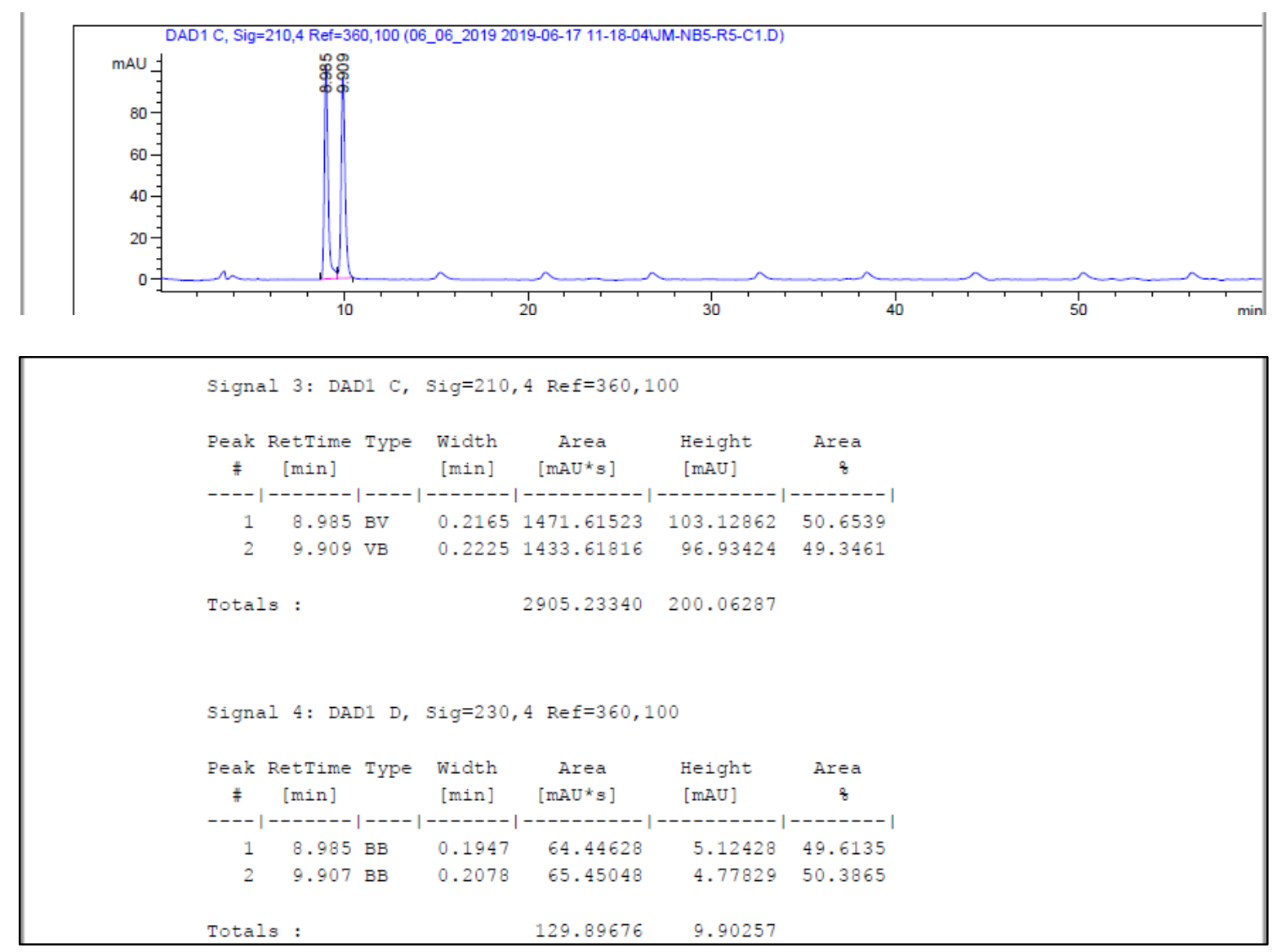

Figure S4. Racemic standard of (S41).

ChiralPak ${ }^{\circledR} \mathrm{IC}, 10 \%$ IPA in Hexanes, 60 min run, $1 \mathrm{~mL} / \mathrm{min}$. 


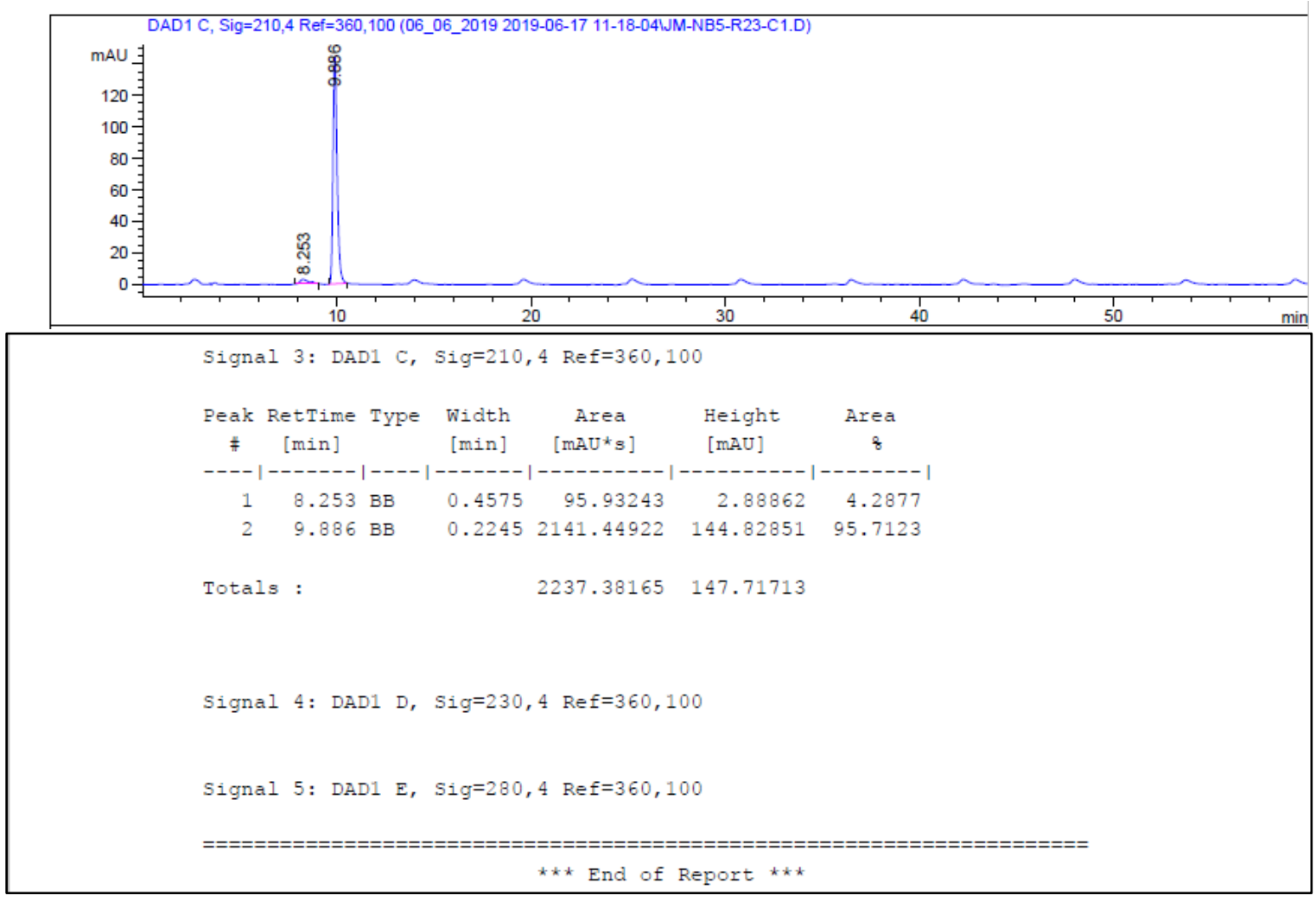

Figure S5. Enantioenriched carboxylic acid (S41; >99\% e.e.). ChiralPak ${ }^{\circledR} \mathrm{IC}, 10 \%$ IPA in Hexanes, 60 min run, $1 \mathrm{~mL} / \mathrm{min}$.

\section{B. Product Characterization}<smiles>O=C(CCc1ccccc1)Cc1ccccc1</smiles>

4-(3-oxo-5,5-diphenylpentyl)benzonitrileone (4) was prepared according to the general procedure $\mathrm{A}$. The title compound was isolated using automated column chromatography eluting with Ether:Hexanes (0\% 4 CV, 0-5\% 12 CV, 10\% 3 CV, 15\% 2 CV, 20\% 2 CV. $25 \% 5 \mathrm{CV}$ ) to produce a white solid (116 $\mathrm{mg}, 68 \%$ yield).

${ }^{1} \mathrm{H}$ NMR $\left(500 \mathrm{MHz}, \mathrm{CDCl}_{3}\right): \delta 7.49(\mathrm{~d}, J=8.2 \mathrm{~Hz}, 2 \mathrm{H}), 7.29-7.22(\mathrm{~m}, 4 \mathrm{H}), 7.21-7.16(\mathrm{~m}, 6 \mathrm{H})$, $7.12(\mathrm{~d}, J=8.2 \mathrm{~Hz}, 2 \mathrm{H}), 4.57(\mathrm{t}, J=7.7 \mathrm{~Hz}, 1 \mathrm{H}), 3.14(\mathrm{~d}, J=7.7 \mathrm{~Hz}, 2 \mathrm{H}), 2.84(\mathrm{t}, J=7.2 \mathrm{~Hz}$, $2 \mathrm{H}), 2.65$ (t, $J=7.2 \mathrm{~Hz}, 2 \mathrm{H})$.

${ }^{13} \mathrm{C}$ NMR $\left(126 \mathrm{MHz} \mathrm{CDCl}_{3}\right): \delta 207.35,146.75,143.68,132.35,129.26,128.77,127.78,126.70$, 119.13, 110.04, 49.10, 46.29, 44.27, 29.38.

HRMS: (ESI-TOF) calculated for $\mathrm{C}_{24} \mathrm{H}_{21} \mathrm{NaO}^{+}\left([\mathrm{M}+\mathrm{Na}]^{+}\right): 362.1515$, found: 362.1512 .

FTIR (ATR cm$\left.^{-1}\right):$ 2923, 2225, 1712, 1605, 1579, 1493, 1450, 1412, 1369, 1241, 1177, 1155, $1091,1074,1030,822,748,696,625,607,550,474,425$.

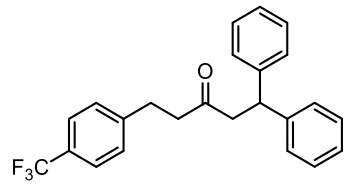


1,1-diphenyl-5-(4-(trifluoromethyl)phenyl)pentan-3-one (5) was prepared according to the general procedure $A$. The title compound was isolated using automated column chromatography eluting with Ether:Hexanes ( $0 \% 4 \mathrm{CV}, 0-10 \% 10 \mathrm{CV}, 10 \% 10 \mathrm{CV}$ ) to produce a white solid (121 $\mathrm{mg}, 63 \%$ yield).

${ }^{1} \mathrm{H}$ NMR $\left(400 \mathrm{MHz}, \mathrm{CDCl}_{3}\right): \delta 7.53(\mathrm{~d}, J=7.8 \mathrm{~Hz}, 2 \mathrm{H}), 7.35-7.29(\mathrm{~m}, 4 \mathrm{H}), 7.28-7.17(\mathrm{~m}, 8 \mathrm{H})$, $4.65(\mathrm{t}, J=7.6 \mathrm{~Hz}, 1 \mathrm{H}), 3.20(\mathrm{~d}, J=7.7 \mathrm{~Hz}, 2 \mathrm{H}), 2.90(\mathrm{t}, J=7.3 \mathrm{~Hz}, 2 \mathrm{H}), 2.70(\mathrm{t}, J=7.3 \mathrm{~Hz}, 2 \mathrm{H})$. ${ }^{13} \mathrm{C}$ NMR $\left(126 \mathrm{MHz}, \mathrm{CD}_{2} \mathrm{Cl}_{2}\right): \delta 207.50,145.95(\mathrm{~d}, \mathrm{~J}=1.5 \mathrm{~Hz}), 129.10,128.95,128.37$ (appd, J $=32.2 \mathrm{~Hz}$ ), 127.96, 126.81, 125.57 (q, J = 3.8 Hz), 124.83 (q, J = 271.7 Hz), 48.96, 46.39, 44.54, 29.41. Note: Carbon peaks for this compound are overlaping, so assignment is missing one carbon peak.

Note: HSQC NMR of (5) in $\mathrm{CD}_{2} \mathrm{Cl}_{2}$.

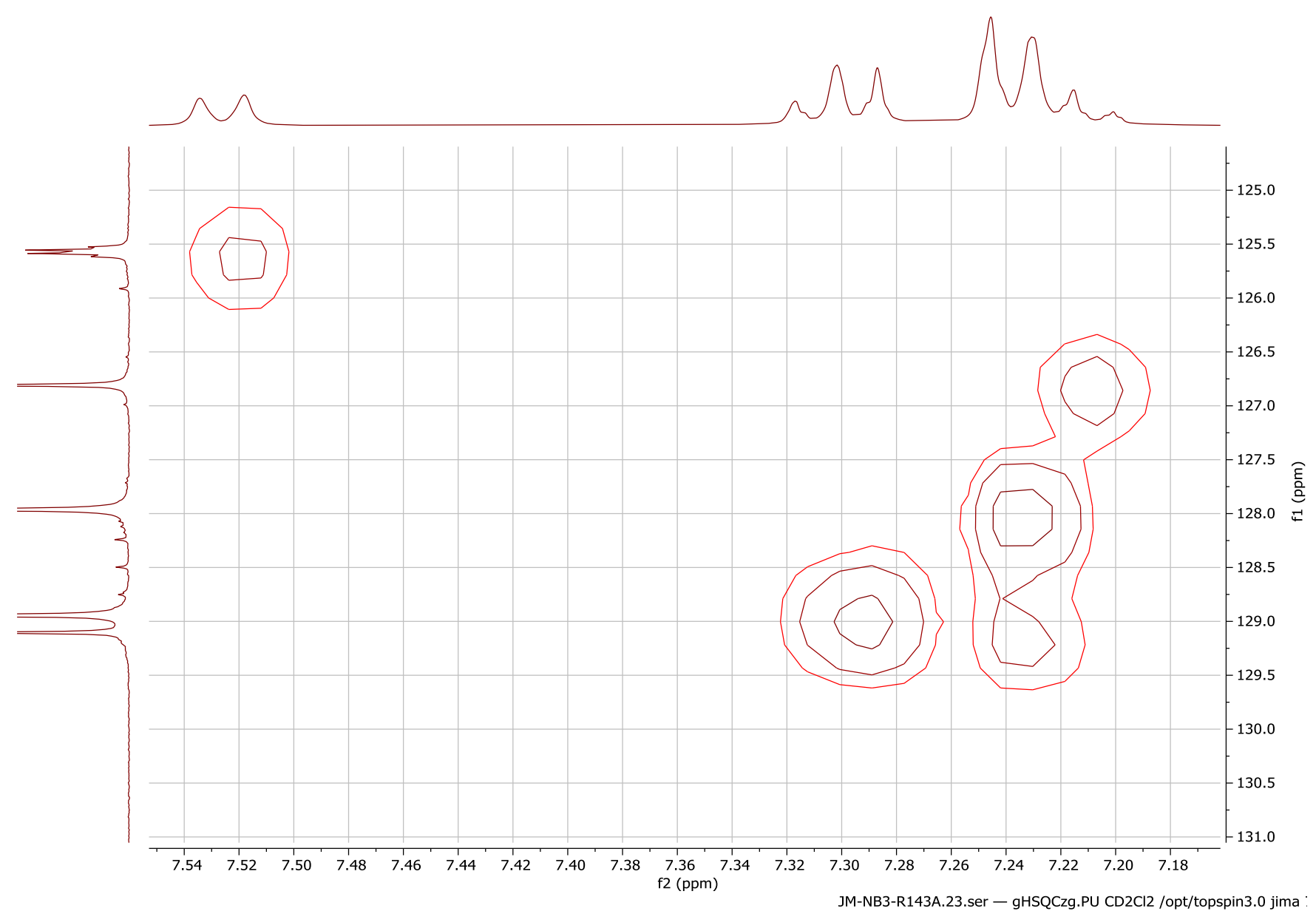

${ }^{19} \mathrm{~F}$ NMR $\left(282 \mathrm{MHz}, \mathrm{CDCl}_{3}\right): \delta-62.39$.

HRMS: (ESI-TOF) calculated for $\mathrm{C}_{24} \mathrm{H}_{21} \mathrm{~F}_{3} \mathrm{NaO}^{+}\left([\mathrm{M}+\mathrm{Na}]^{+}\right): 405.1437$, found: 405.1435 . FTIR (ATR cm ${ }^{-1}$ ): 3027, 1713, 1617, 1599, 1494, 1450, 1417, 1322, 1161, 1109, 1065, 1030, $1017,907,825,791,729,697,648,617,603,573,554,516,471,413$.<smiles>COc1ccc(CCC(=O)c2ccccc2C2Oc3ccccc32)cc1</smiles>

methyl 4-(3-oxo-5,5-diphenylpentyl)benzoate (6) was prepared according to the general procedure $\mathrm{A}$. The title compound was isolated using automated column chromatography eluting with Ether:Hexanes (0\% $7 \mathrm{CV}, 0-5 \% 12 \mathrm{CV}, 5 \% 2 \mathrm{CV}, 5-10 \% 4 \mathrm{CV}, 10 \% 2 \mathrm{CV}, 15 \% 8 \mathrm{CV}$ ) to produce a white solid (121 $\mathrm{mg}, 65 \%$ yield). 
${ }^{1} \mathrm{H}$ NMR $\left(500 \mathrm{MHz}, \mathrm{CDCl}_{3}\right): \delta 7.96(\mathrm{~d}, J=8.3 \mathrm{~Hz}, 2 \mathrm{H}), 7.36-7.30(\mathrm{~m}, 4 \mathrm{H}), 7.30-7.21(\mathrm{~m}, 6 \mathrm{H})$, $7.20-7.15(\mathrm{~m}, 2 \mathrm{H}), 4.65(\mathrm{t}, J=7.6 \mathrm{~Hz}, 1 \mathrm{H}), 3.96(\mathrm{~s}, 3 \mathrm{H}), 3.21(\mathrm{~d}, J=7.6 \mathrm{~Hz}, 2 \mathrm{H}), 2.89$ (t, $J=$ $7.4 \mathrm{~Hz}, 2 \mathrm{H}), 2.71(\mathrm{t}, J=7.4 \mathrm{~Hz}, 2 \mathrm{H})$.

${ }^{13} \mathrm{C} \mathrm{NMR}\left(126 \mathrm{MHz}, \mathrm{CDCl}_{3}\right): 0207.68,167.14,146.56,143.79,129.89,128.73,128.43,128.12$, 127.78, 126.62, 52.15, 49.12, 46.19, 44.59, 29.40.

HRMS: (ESI-TOF) calculated for $\mathrm{C}_{25} \mathrm{H}_{25} \mathrm{O}_{3}{ }^{+}\left([\mathrm{M}+\mathrm{H}]^{+}\right): 373.1798$, found: 373.1794 .

FTIR (ATR cm$\left.^{-1}\right):$ 1703, 1607, 1435, 1277, 1255, 1179, 1104, 1093, 1076, 1030, 1019, 986, $757,749,727,699,628,606,571,551,511,486,471$.

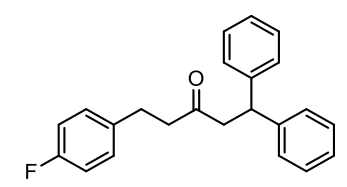

5-(4-fluorophenyl)-1,1-diphenylpentan-3-one (7) was prepared according to the general procedure $\mathrm{A}$. The title compound was isolated using automated column chromatography eluting with Ether:Hexanes ( $0 \% 4 \mathrm{CV}, 0-10 \% 10 \mathrm{CV}, 10 \% 12 \mathrm{CV}$ ) to produce a yellow white solid (123 $\mathrm{mg}, 74 \%$ yield).

1 ${ }^{1}$ NMR $\left(500 ~ M H z, \mathbf{C D C l}_{3}\right): \delta 7.36-7.29(\mathrm{~m}, 5 \mathrm{H}), 7.28-7.21(\mathrm{~m}, 5 \mathrm{H}), 7.10-7.03(\mathrm{~m}, 2 \mathrm{H})$, $7.01-6.93(\mathrm{~m}, 2 \mathrm{H}), 4.65(\mathrm{t}, \mathrm{J}=7.6 \mathrm{~Hz}, 1 \mathrm{H}), 3.20(\mathrm{~d}, \mathrm{~J}=7.6 \mathrm{~Hz}, 2 \mathrm{H}), 2.82(\mathrm{t}, \mathrm{J}=7.4 \mathrm{~Hz}, 2 \mathrm{H})$, $2.68(\mathrm{t}, \mathrm{J}=7.4 \mathrm{~Hz}, 2 \mathrm{H})$.

${ }^{13}$ C NMR (126 MHz, CDCl 3 ): $\delta$ 207.99, $161.44(\mathrm{~d}, J=243.7 \mathrm{~Hz}), 143.88,136.67$ (d, $\left.J=3.2 \mathrm{~Hz}\right)$, $129.79(\mathrm{~d}, J=7.8 \mathrm{~Hz}), 128.74,127.83,126.62,115.30(\mathrm{~d}, J=21.1 \mathrm{~Hz}), 49.21,46.17,45.21$ (d, $J=1.1 \mathrm{~Hz}), 28.67$.

${ }^{19} \mathrm{~F}$ NMR (282 $\left.\mathrm{MHz}, \mathrm{CDCl}_{3}\right): \delta-117.39$.

HRMS: (ESI-TOF) calculated for $\mathrm{C}_{23} \mathrm{H}_{22} \mathrm{FO}^{+}\left([\mathrm{M}+\mathrm{H}]^{+}\right)$: 333.1649, found: 333.1643.

FTIR (ATR cm-1): 1701, 1660, 1593, 1509, 1355, 1344, 1253, 1220, 836, 812, 749, 701.

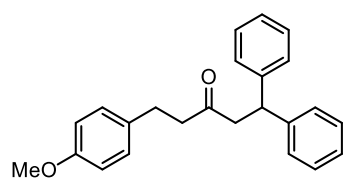

5-(4-methoxyphenyl)-1,1-diphenylpentan-3-one (8) was prepared according to the general procedure $\mathrm{A}$. The title compound was isolated using automated column chromatography eluting with Ether:Hexanes (0\% $4 \mathrm{CV}, 0-10 \% 10 \mathrm{CV}, 10 \% 7 \mathrm{CV}$ ) to produce a white solid $(140 \mathrm{mg}, 81 \%$ yield).

1H NMR $\left(500 \mathrm{MHz}^{1} \mathrm{CDCl}_{3}\right): \delta 7.35-7.30(\mathrm{~m}, 4 \mathrm{H}), 7.27-7.20(\mathrm{~m}, 6 \mathrm{H}), 7.06-7.03(\mathrm{~m}, 2 \mathrm{H})$, $6.86-6.82(\mathrm{~m}, 2 \mathrm{H}), 4.66(\mathrm{t}, J=7.6 \mathrm{~Hz}, 1 \mathrm{H}), 3.83(\mathrm{~s}, 3 \mathrm{H}), 3.19(\mathrm{~d}, J=7.5 \mathrm{~Hz}, 2 \mathrm{H}), 2.79(\mathrm{t}, J=$ $7.5 \mathrm{~Hz}, 2 \mathrm{H}), 2.69-2.62(\mathrm{~m}, 2 \mathrm{H})$.

${ }^{13} \mathrm{C} \mathrm{NMR}\left(126 \mathrm{MHz}, \mathrm{CDCl}_{3}\right): \delta 208.30,158.00,143.96,133.07,129.31,128.70,127.83,126.55$, 113.96, 55.36, 49.20, 46.10, 45.43, 28.70.

HRMS: (ESI-TOF) calculated for $\mathrm{C}_{24} \mathrm{H}_{24} \mathrm{NaO}_{2}{ }^{+}\left([\mathrm{M}+\mathrm{Na}]^{+}\right): 367.1668$, found: 367.1660 .

FTIR (ATR m$\left.^{-1}\right):$ 2915, 1702, 1607, 1580, 1511, 1492, 1464, 1448, 1401, 1366, 1317, 1298, 1240, 1176, 1091, 1076, 1028, 854, 828, 806, 788, 768, 742, 700, 625, 607, 571, 557, 543, 528, 518, 468, 436, 408, 401.

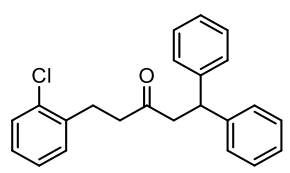


5-(2-chlorophenyl)-1,1-diphenylpentan-3-one (9) was prepared according to the general procedure $\mathrm{A}$. The title compound was isolated using automated column chromatography eluting with Ether:Hexanes ( $0 \% 7 \mathrm{CV}, 0-5 \% 10 \mathrm{CV}, 5 \% 6 \mathrm{CV}$ ) to produce a yellow tinted solid (110 mg, $63 \%$ yield).

${ }^{1} \mathrm{H}$ NMR $\left(500 \mathrm{MHz}, \mathrm{CDCl}_{3}\right): \delta 7.32-7.24(\mathrm{~m}, 5 \mathrm{H}), 7.23-7.15(\mathrm{~m}, 6 \mathrm{H}), 7.11$ (ddd, $J=12.4,6.0$, $2.6 \mathrm{~Hz}, 3 \mathrm{H}$ ), 4.60 (t, $J=7.6 \mathrm{~Hz}, 1 \mathrm{H}$ ), 3.15 (d, $J=7.6 \mathrm{~Hz}, 2 \mathrm{H}$ ), 2.89 (t, $J=7.6 \mathrm{~Hz}, 2 \mathrm{H}$ ), 2.65 (t, $J$ $=7.6 \mathrm{~Hz}, 2 \mathrm{H}$ ).

${ }^{13} \mathrm{C}$ NMR $\left(126 \mathrm{MHz}, \mathrm{CDCl}_{3}\right): \delta 207.99,143.89,138.61,133.95,130.75,129.62,128.74,127.84$, 127.77, 127.01, 126.61, 49.06, 46.28, 43.21, 27.67.

HRMS: (ESI-TOF) calculated for $\mathrm{C}_{23} \mathrm{H}_{22} \mathrm{ClO}^{+}\left([\mathrm{M}+\mathrm{H}]^{+}\right)$: 349.1354 , found: 349.1351 .

FTIR (ATR cm ${ }^{-1}$ ): 3025, 2931, 2892, 1703, 1599, 1492, 1473, 1445, 1414, 1367, 1305, 1268 , 1232, 1196, 1077, 1050, 1034, 1000, 991, 753, 742, 727, 719, 697, 665, 624, 610, 582, 557, 523, 483, 457, 436.

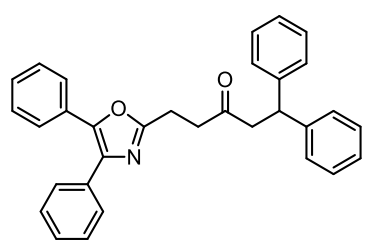

5-(4,5-diphenyloxazol-2-yl)-1,1-diphenylpentan-3-one (10) was prepared according to the general Procedure A using 2.4 equivalents of phosphine and at a concentration of $0.33 \mathrm{M}$. The title compound was isolated using automated column chromatography eluting with Ether:Hexanes $(0 \% 5 \mathrm{CV}, 5-20 \% 18 \mathrm{CV}, 20 \% 5 \mathrm{CV}$ ) to produce a white solid (228 mg, 34\% yield).

${ }^{1} \mathrm{H}$ NMR $\left(500 \mathrm{MHz}, \mathrm{CDCl}_{3}\right): \delta 7.63(\mathrm{~d}, J=6.8 \mathrm{~Hz}, 2 \mathrm{H}), 7.57(\mathrm{~d}, J=6.9 \mathrm{~Hz}, 2 \mathrm{H}), 7.42-7.31(\mathrm{~m}$, $6 \mathrm{H}), 7.31-7.24(\mathrm{~m}, 8 \mathrm{H}), 7.22-7.16(\mathrm{~m}, 2 \mathrm{H}), 4.69(\mathrm{t}, J=7.5 \mathrm{~Hz}, 1 \mathrm{H}), 3.32(\mathrm{~d}, J=7.7 \mathrm{~Hz}, 2 \mathrm{H})$, $3.07(\mathrm{t}, J=7.1 \mathrm{~Hz}, 2 \mathrm{H}), 2.97(\mathrm{t}, J=7.2 \mathrm{~Hz}, 2 \mathrm{H})$.

${ }^{13} \mathrm{C}$ NMR $\left(126 \mathrm{MHz} \mathbf{C D C l}_{3}\right): \delta 206.76,162.31,145.43,143.88,135.12,132.59,129.07,128.72$, 128.66, 128.51, 128.14, 128.00, 127.80, 126.58, 126.55, 49.06, 45.98, 39.76, 22.10.

Note: Carbon peaks for this compound are overlaping, so assignment is missing one carbon peak.

HRMS: (ESI-TOF) calculated for $\mathrm{C}_{32} \mathrm{H}_{27} \mathrm{NNaO}_{2}{ }^{+}\left([\mathrm{M}+\mathrm{Na}]^{+}\right): 480.1935$, found: 480.1934 .

FTIR (ATR cm$\left.^{-1}\right): 3026,2921,1716,1569,1493,1447,1365,1264,1218,1156,1094,1072$, 1057, 1024, 1000, 961, 913, 762, 733, 692, 673, 625, 606, 572, 555, 522, 470.

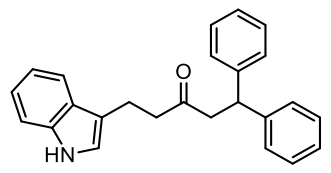

5-(1 H-indol-3-yl)-1,1-diphenylpentan-3-one (11) was prepared according to the general procedure A using 2.4 equivalents of phosphine and at a concentration of $0.33 \mathrm{M}$. The title compound was isolated using automated column chromatography eluting with Ether:Hexanes (10\% $4 \mathrm{CV}, 10-20 \% 6.8 \mathrm{CV}, 20 \% 10 \mathrm{CV}$ ) to produce a yellow white solid ( $106.2 \mathrm{mg}, 60 \%$ yield).

1 $\mathrm{H}$ NMR $\left(500 \mathrm{MHz} \mathrm{CDCl}_{3}\right): \delta 7.88(\mathrm{bs}, 1 \mathrm{H}), 7.50(\mathrm{~d}, \mathrm{~J}=7.4 \mathrm{~Hz}, 1 \mathrm{H}), 7.33(\mathrm{~d}, \mathrm{~J}=8.2 \mathrm{~Hz}, 1 \mathrm{H})$, $7.29-7.24(\mathrm{~m}, 4 \mathrm{H}), 7.21-7.16(\mathrm{~m}, 7 \mathrm{H}), 7.14-7.02(\mathrm{~m}, 1 \mathrm{H}), 6.78(\mathrm{~d}, \mathrm{~J}=2.3 \mathrm{~Hz}, 1 \mathrm{H}), 4.61(\mathrm{t}$, $\mathrm{J}=7.6 \mathrm{~Hz}, 1 \mathrm{H}), 3.15(\mathrm{~d}, \mathrm{~J}=7.6 \mathrm{~Hz}, 2 \mathrm{H}), 2.96(\mathrm{t}, \mathrm{J}=7.6 \mathrm{~Hz}, 2 \mathrm{H}), 2.74(\mathrm{t}, \mathrm{J}=7.4 \mathrm{~Hz}, 2 \mathrm{H})$.

${ }^{13} \mathrm{C} \mathrm{NMR}\left(126 \mathrm{MHz}, \mathrm{CDCl}_{3}\right): \delta 208.98,143.98,136.31,128.68,127.84,127.18,126.52,122.10$, 121.61, 119.36, 118.76, 115.09, 111.25, 49.10, 46.12, 44.05, 19.17.

HRMS: (ESI-TOF) calculated for $\mathrm{C}_{25} \mathrm{H}_{23} \mathrm{NNaO}^{+}\left([\mathrm{M}+\mathrm{Na}]^{+}\right): 376.1672$, found: 376.1678 . 
FTIR (ATR cm $\left.{ }^{-1}\right): 2360,1707,1493,1455,1418,1338,1264,1092,1030,1010,731,697,608$, $580,552,470,423$.<smiles>O=C(Cc1cccs1)CC(c1ccccc1)c1ccccc1</smiles>

1,1-diphenyl-5-(thiophen-2-yl)pentan-3-one (12) was prepared according to the general procedure $\mathrm{A}$. The title compound was isolated using automated column chromatography eluting with EtOAc:Hexanes ( $0 \% 4 \mathrm{CV}, 1 \% 4 \mathrm{CV}, 1-7 \% 9 \mathrm{CV}, 7 \% 13 \mathrm{CV}$ ) to produce a yellow oil (109 $\mathrm{mg}, 68 \%$ yield).

1H NMR (500 MHz, CDCl 3$): \delta 7.30-7.24(\mathrm{~m}, 4 \mathrm{H}), 7.23-7.15(\mathrm{~m}, 6 \mathrm{H}), 7.09(\mathrm{dd}, J=5.1,1.3$ $\mathrm{Hz}, 1 \mathrm{H}), 6.87(\mathrm{dd}, J=5.2,3.4 \mathrm{~Hz}, 1 \mathrm{H}), 6.68(\mathrm{~d}, J=3.4 \mathrm{~Hz}, 1 \mathrm{H}), 4.61(\mathrm{td}, J=7.6,2.4 \mathrm{~Hz}, 1 \mathrm{H})$, 3.17 (dd, $J=7.6,1.6 \mathrm{~Hz}, 2 \mathrm{H}$ ), 3.01 (t, $J=7.4 \mathrm{~Hz}, 2 \mathrm{H}), 2.70$ (t, $J=7.4 \mathrm{~Hz}, 2 \mathrm{H}$ ).

${ }^{13} \mathrm{C} \mathrm{NMR}\left(126 \mathrm{MHz}, \mathrm{CDCl}_{3}\right): \delta 207.53,143.89,143.66,128.74,127.83,126.94,126.61,124.69$, 123.44, 49.18, 46.08, 45.27, 23.75.

HRMS: (ESI-TOF) calculated for $\mathrm{C}_{21} \mathrm{H}_{20} \mathrm{NaOS}^{+}\left([\mathrm{M}+\mathrm{Na}]^{+}\right): 343.1127$, found: 343.1122 .

FTIR (ATR cm $\left.{ }^{-1}\right): 3025,2921,1711,1598,1492,1449,1406,1368,1235,1091,1073,1030$, 1003, 847, 821, 744, 692, 626, 609, 548, 502, 470.

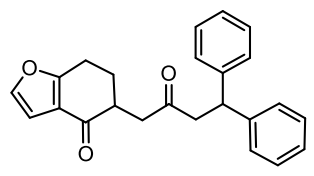

5-(2-oxo-4,4-diphenylbutyl)-6,7-dihydrobenzofuran-4(5H)-one (13) was prepared according to the general procedure $A$. The title compound was isolated using automated column chromatography eluting with Ether:Hexanes ( $0 \% 4 \mathrm{CV}, 0-15 \% 8 \mathrm{CV}, 15 \% 15 \mathrm{CV})$ to produce a white solid (68 $\mathrm{mg}, 38 \%$ yield). Crude NMR analysis shows a $52 \%$ Yield.

${ }^{1} \mathrm{H}$ NMR $\left(500 \mathbf{M H z}_{2} \mathbf{C D C l}_{3}\right): \delta 7.30-7.21(\mathrm{~m}, 9 \mathrm{H}), 7.20-7.15(\mathrm{~m}, 2 \mathrm{H}), 6.62(\mathrm{~d}, J=2.0 \mathrm{~Hz}, 1 \mathrm{H})$, $4.62(\mathrm{t}, J=7.7 \mathrm{~Hz}, 1 \mathrm{H}), 3.28(\mathrm{qd}, J=16.4,7.7 \mathrm{~Hz}, 2 \mathrm{H}), 3.13(\mathrm{dd}, J=17.6,4.7 \mathrm{~Hz}, 1 \mathrm{H}), 2.96-$ $2.75(\mathrm{~m}, 3 \mathrm{H}), 2.26(\mathrm{dd}, J=17.6,7.5 \mathrm{~Hz}, 1 \mathrm{H}), 2.04-1.96(\mathrm{~m}, 1 \mathrm{H}), 1.71$ (tdd, $J=12.9,11.4,5.7$ $\mathrm{Hz}, 1 \mathrm{H})$.

${ }^{13} \mathrm{C} \mathrm{NMR}\left(126 \mathrm{MHz}, \mathrm{CDCl}_{3}\right): \delta 207.64,194.82,166.67,144.02,143.85,142.95,128.73,127.93$, 127.86, 126.61, 120.68, 106.83, 49.28, 46.28, 43.15, 42.58, 28.79, 23.49.

HRMS: (ESI-TOF) calculated for $\mathrm{C}_{24} \mathrm{H}_{22} \mathrm{NaO}_{3}{ }^{+}\left([\mathrm{M}+\mathrm{Na}]^{+}\right): 381.1461$, found: 381.1457 . FTIR (ATR cm$\left.^{-1}\right):$ 2922, 2853, 1772, 1713, 1671, 1594, 1579, 1493, 1451, 1372, 1288, 1261, 1196, 1155, 1117, 1031, 947, 883, 773, 735, 696, 607, 558, 535, 474, 447, 403. 


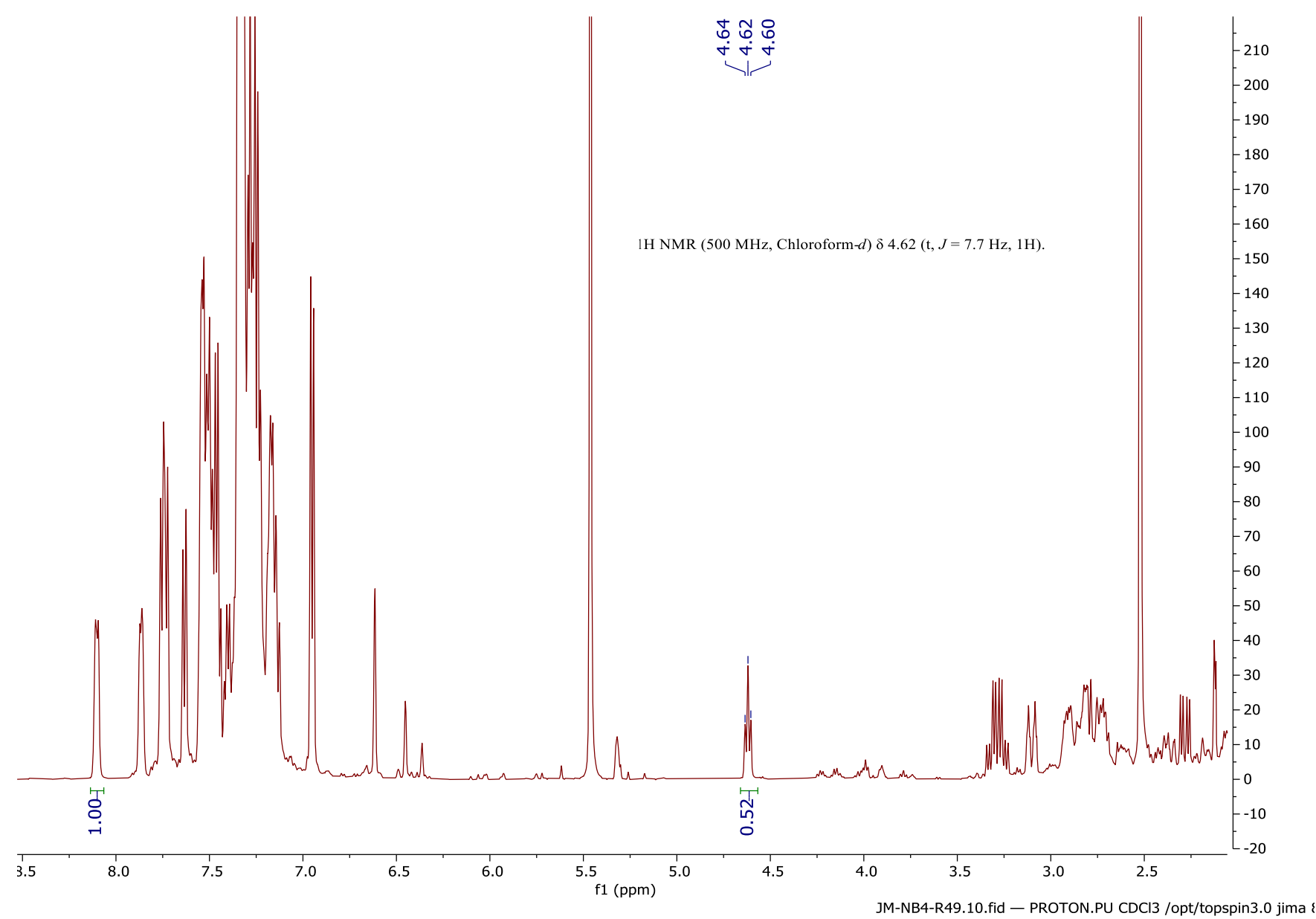

Figure S6. Crude NMR Yield for (13). Referenced to one equivalent of 1-fluoronaphthalene.<smiles>O=C(Cc1ccccc1C1Oc2ccccc21)CC(F)(F)F</smiles>

6,6,6-trifluoro-1,1-diphenylhexan-3-one (14) was prepared according to the general procedure $\mathrm{A}$. The title compound was isolated using automated column chromatography eluting with Ether:Hexanes ( $0 \% 4 \mathrm{CV}, 0-4 \% 9 \mathrm{CV}, 6 \% 7 \mathrm{CV}$ ) to produce a yellow oil $(93 \mathrm{mg}, 61 \%$ yield).

${ }^{1} \mathrm{H}$ NMR $\left(500 \mathrm{MHz}, \mathrm{CDCl}_{3}\right): \delta 7.30-7.26(\mathrm{~m}, 4 \mathrm{H}), 7.24-7.16(\mathrm{~m}, 6 \mathrm{H}), 4.59(\mathrm{t}, J=7.6 \mathrm{~Hz}, 1 \mathrm{H})$, $3.21(\mathrm{~d}, J=7.6 \mathrm{~Hz}, 2 \mathrm{H}), 2.59-2.53(\mathrm{~m}, 2 \mathrm{H}), 2.29$ (qt, $\mathrm{J}=10.8,7.7 \mathrm{~Hz}, 2 \mathrm{H})$.

${ }^{13} \mathrm{C} \mathrm{NMR}\left(126 \mathrm{MHz}, \mathrm{CDCl}_{3}\right): 0205.37,143.55,128.82,127.73,126.95$ (q, J = 275.8 Hz), 126.77, $48.90,46.20,35.91(\mathrm{q}, \mathrm{J}=2.6 \mathrm{~Hz}), 27.81$ (q, J = 29.9 Hz).

${ }^{19} \mathrm{~F} \mathrm{NMR}\left(376 \mathrm{MHz}, \mathrm{CDCl}_{3}\right): \delta-66.68(\mathrm{t}, J=10.7 \mathrm{~Hz})$.

HRMS: (ESI-TOF) calculated for $\mathrm{C}_{18} \mathrm{H}_{17} \mathrm{~F}_{3} \mathrm{NaO}^{+}\left([\mathrm{M}+\mathrm{Na}]^{+}\right): 329.1124$, found: 329.1126 .

FTIR (ATR cm ${ }^{-1}$ ): 3028, 1720, 1597, 1494, 1452, 1439, 1423, 1379, 1314, 1255, 1233, 1195, $1175,1134,1101,1089,1001,983,927,850,823,794,746,726,696,627,602,565,538,469$, 458, 403.

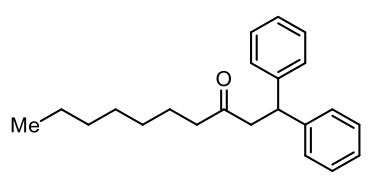


1,1-diphenyldecan-3-one (15) was prepared according to the general procedure A. The title compound was isolated using automated column chromatography eluting with Ether:Hexanes ( $0 \% 4 \mathrm{CV}, 0-5 \% 8 \mathrm{CV}, 5 \% 6 \mathrm{CV}$ ) to produce a yellow oil (107 $\mathrm{mg}, 69 \%$ yield).

${ }^{1} \mathrm{H}$ NMR $\left(500 \mathrm{MHz} \mathrm{CDCl}_{3}\right): \delta 7.32-7.23(\mathrm{~m}, 8 \mathrm{H}), 7.22-7.18(\mathrm{~m}, 2 \mathrm{H}), 4.65(\mathrm{t}, J=7.5 \mathrm{~Hz}, 1 \mathrm{H})$, $3.18(\mathrm{~d}, J=7.5 \mathrm{~Hz}, 2 \mathrm{H}), 2.33(\mathrm{t}, J=7.4 \mathrm{~Hz}, 2 \mathrm{H}), 1.50(\mathrm{p}, J=7.3 \mathrm{~Hz}, 2 \mathrm{H}), 1.34-1.13(\mathrm{~m}, 8 \mathrm{H})$, $0.90(\mathrm{t}, J=7.0 \mathrm{~Hz}, 3 \mathrm{H})$.

${ }^{13} \mathrm{C} \mathrm{NMR}\left(126 \mathrm{MHz}, \mathrm{CDCl}_{3}\right): \delta 209.30,144.09,128.63,127.84,126.48,48.86,46.08,43.72$, 31.73, 29.11, 23.62, 22.69, 14.18.

Note: 13 carbon signals observed due to overlap.

HRMS: (ESI-TOF) calculated for $\mathrm{C}_{22} \mathrm{H}_{28} \mathrm{NaO}^{+}\left([\mathrm{M}+\mathrm{Na}]^{+}\right): 331.2032$, found: 331.2029 .

FTIR (ATR cm $\left.{ }^{-1}\right):$ 2924, 2853, 1711, 1493, 1450, 1368, 1068, 1030, 744, 696, 608, 575, 555.

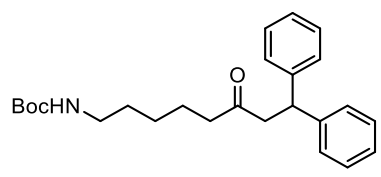

tert-butyl (6-oxo-8,8-diphenyloctyl)carbamate (16) was prepared according to the general procedure $\mathrm{A}$. The title compound was isolated using automated column chromatography eluting with EtOAc:Hexanes (5\% $6 \mathrm{CV}, 5-20 \% 10 \mathrm{CV}, 20 \% 7 \mathrm{CV}$ ) to produce a white solid (198 mg, $82 \%$ yield).

1H NMR (500 MHz, CDCl 3 ): $\delta 7.26(\mathrm{t}, J=3.7 \mathrm{~Hz}, 4 \mathrm{H}), 7.24-7.19(\mathrm{~m}, 4 \mathrm{H}), 7.17$ (td, $J=6.9,1.5$ $\mathrm{Hz}, 2 \mathrm{H}), 4.59(\mathrm{t}, J=7.6 \mathrm{~Hz}, 1 \mathrm{H}), 4.46(\mathrm{bs}, 1 \mathrm{H}), 3.14(\mathrm{~d}, J=7.6 \mathrm{~Hz}, 2 \mathrm{H}), 3.04(\mathrm{q}, J=6.8 \mathrm{~Hz}, 2 \mathrm{H})$, $2.31(\mathrm{t}, J=7.2 \mathrm{~Hz}, 2 \mathrm{H}), 1.54-1.45(\mathrm{~m}, 2 \mathrm{H}), 1.44(\mathrm{~s}, 9 \mathrm{H}) 1.37(\mathrm{p}, J=7.3 \mathrm{~Hz}, 2 \mathrm{H}), 1.14$ (p, $J=$ $7.7 \mathrm{~Hz}, 2 \mathrm{H})$.

${ }^{13} \mathrm{C}$ NMR (126 MHz, $\left.\mathrm{CDCl}_{3}\right):$ $\delta 209.11,156.07,144.03,128.71,127.87,126.58,79.20,48.94$, 46.21, 43.54, 40.48, 29.92, 28.57, 26.32, 23.17.

HRMS: (ESI-TOF) calculated for $\mathrm{C}_{25} \mathrm{H}_{33} \mathrm{NNaO}_{3}{ }^{+}\left([\mathrm{M}+\mathrm{Na}]^{+}\right): 418.2353$, found: 418.2360 .

FTIR (ATR cm$\left.^{-1}\right)$ : 3356, 3026, 2929, 2860, 1697, 1599, 1494, 1450, 1390, 1364, 1246, 1165, 1064, 1031, 987, 866, 780, 746, 697, 626, 608, 575, 555, 468.

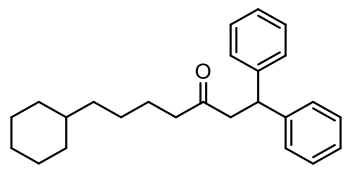

7-cyclohexyl-1,1-diphenylheptan-3-one (17) was prepared according to the general procedure $\mathrm{B}$. The title compound was isolated using automated column chromatography eluting with Ether:Hexanes $(0 \% 4 \mathrm{CV}, 0-10 \% 10 \mathrm{CV}, 10 \% 10 \mathrm{CV})$ to produce a slightly yellow tinted white solid $(149.8 \mathrm{mg}, 86 \%$ yield)

1H NMR (500 MHz, CDCl 3 ): $\delta 7.30-7.24(\mathrm{~m}, 4 \mathrm{H}), 7.24-7.20(\mathrm{~m}, 4 \mathrm{H}), 7.17(\mathrm{t}, \mathrm{J}=7.2 \mathrm{~Hz}, 2 \mathrm{H})$, $4.61(\mathrm{t}, \mathrm{J}=7.6 \mathrm{~Hz}, 1 \mathrm{H}), 3.15(\mathrm{~d}, \mathrm{~J}=7.5 \mathrm{~Hz}, 2 \mathrm{H}), 2.30(\mathrm{t}, \mathrm{J}=7.4 \mathrm{~Hz}, 2 \mathrm{H}), 1.75-1.59(\mathrm{~m}, 5 \mathrm{H})$, $1.44(\mathrm{p}, \mathrm{J}=7.4 \mathrm{~Hz}, 2 \mathrm{H}), 1.27-1.03(\mathrm{~m}, 8 \mathrm{H}), 0.80(\mathrm{q}, \mathrm{J}=13.9,12.1 \mathrm{~Hz}, 2 \mathrm{H})$.

${ }^{13} \mathrm{C}$ NMR $\left(126 \mathrm{MHz}, \mathrm{CDCl}_{3}\right)$ : $\delta$ 209.42, 144.13, 128.68, 127.88, 126.53, 48.93, 46.11, 43.81, 37.57, 37.30, 33.49, 26.85, 26.54, 26.45, 23.96.

HRMS: (ESI-TOF) calculated for $\mathrm{C}_{25} \mathrm{H}_{33} \mathrm{O}^{+}\left([\mathrm{M}+\mathrm{H}]^{+}\right)$: 349.2526 , found: 349.2531.

FTIR (ATR cm$\left.^{-1}\right): 3027,1713,1617,1599,1494,1450,1417,1322,1161,1109,1065,1030$, 1017, 907, 825, 791, 729, 697, 648, 617, 603, 573, 554, 516, 471, 413. 


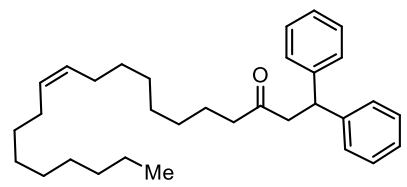

(Z)-1,1-diphenylicos-11-en-3-one (18) was prepared according to the general procedure B. The title compound was isolated using automated column chromatography eluting with Ether:Hexanes ( $0 \% 7 \mathrm{CV}, 0-2 \% 4 \mathrm{CV}, 2-7 \% 5 \mathrm{CV}, 7 \% 7 \mathrm{CV})$ to produce a yellow oil $(223 \mathrm{mg}$, $78 \%$ yield).

${ }^{1} \mathrm{H}$ NMR $\left(500 \mathrm{MHz}, \mathrm{CDCl}_{3}\right): \delta 7.30-7.24(\mathrm{~m}, 4 \mathrm{H}), 7.24-7.20(\mathrm{~m}, 4 \mathrm{H}), 7.19-7.14(\mathrm{~m}, 2 \mathrm{H})$, $5.34(\mathrm{dq}, \mathrm{J}=5.6,5.0 \mathrm{~Hz}, 2 \mathrm{H}), 4.61(\mathrm{t}, J=7.5 \mathrm{~Hz}, 1 \mathrm{H}), 3.15(\mathrm{~d}, J=7.6 \mathrm{~Hz}, 2 \mathrm{H}), 2.30$ (t, $J=7.4$ $\mathrm{Hz}, 2 \mathrm{H}), 2.00(\mathrm{p}, J=6.9 \mathrm{~Hz}, 4 \mathrm{H}), 1.46(\mathrm{p}, J=7.4 \mathrm{~Hz}, 2 \mathrm{H}), 1.38-1.09(\mathrm{~m}, 20 \mathrm{H}), 0.88(\mathrm{t}, J=6.8$ $\mathrm{Hz}, 3 \mathrm{H})$.

${ }^{13} \mathrm{C}$ NMR $\left(126 \mathrm{MHz}, \mathrm{CDCl}_{3}\right): \delta 209.35,144.12,130.12,129.91,128.68,127.88,126.53,48.91$, $46.13,43.77,32.05,29.91$, 29.82, 29.67, 29.47, 29.39, 29.22, 29.17, 27.36, 27.31, 23.65, 22.83, 14.27.

Note: 23 carbon signals observed due to overlap.

HRMS: (ESI-TOF) calculated for $\mathrm{C}_{32} \mathrm{H}_{47} \mathrm{O}^{+}\left([\mathrm{M}+\mathrm{H}]^{+}\right)$: 447.3621, found: 447.3617 .

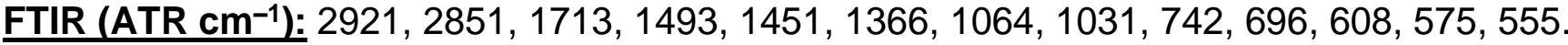<smiles></smiles>

4,4-diphenylbutan-2-one (19) was prepared according to the general procedure $\mathrm{B}$. The title compound was isolated using automated column chromatography eluting with Ether:Hexanes ( $0 \% 8 \mathrm{CV}, 0-8 \% 12 \mathrm{CV}, 15 \% 3 \mathrm{CV})$ to produce a yellow oil $(86.1 \mathrm{mg}, 76 \%$ yield).

${ }^{1} \mathrm{H}$ NMR $\left(500 \mathrm{MHz}, \mathrm{CDCl}_{3}\right): \delta 7.31-7.26(\mathrm{~m}, 4 \mathrm{H}), 7.25-7.22(\mathrm{~m}, 4 \mathrm{H}), 7.20-7.15(\mathrm{~m}, 2 \mathrm{H})$, $4.60(\mathrm{t}, \mathrm{J}=7.6 \mathrm{~Hz}, 1 \mathrm{H}), 3.19(\mathrm{~d}, \mathrm{~J}=7.5 \mathrm{~Hz}, 2 \mathrm{H}), 2.09(\mathrm{~s}, 3 \mathrm{H})$.

${ }^{13} \mathrm{C}$ NMR $\left(126 \mathrm{MHz}, \mathrm{CDCl}_{3}\right): \delta 207.02,143.96,128.72,127.83,126.58,49.80,46.15,30.81$. HRMS: (ESI-TOF) calculated for $\mathrm{C}_{16} \mathrm{H}_{16} \mathrm{NaO}^{+}\left([\mathrm{M}+\mathrm{Na}]^{+}\right)$: 247.1093 , found: 247.1089 .

FTIR (ATR cm$^{-1}$ ): 3024, 2926, 1706, 1595, 1583, 1491, 1450, 1407, 1368, 1332, 1249, 1201, 1162, 1086, 1050, 1031, 1017, 966, 776, 745, 694, 633, 617, 596, 565, 543, 469.

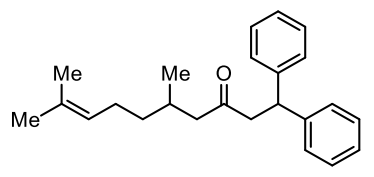

5,9-dimethyl-1,1-diphenyldec-8-en-3-one (20) was prepared according to the general procedure $\mathrm{B}$. The title compound was isolated using automated column chromatography eluting with Ether:Hexanes ( $0 \% 8 \mathrm{CV}, 0-7 \% 10 \mathrm{CV}, 7 \% 3 \mathrm{CV}$ ) to produce a pale yellow oil $(69 \mathrm{mg}, 41 \%$ yield).

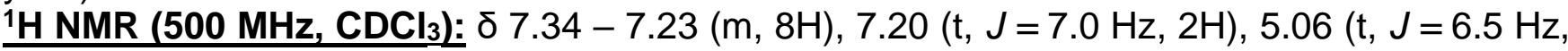
$1 \mathrm{H}), 4.64(\mathrm{t}, J=7.6 \mathrm{~Hz}, 1 \mathrm{H}), 3.17(\mathrm{dd}, J=7.5,1.6 \mathrm{~Hz}, 2 \mathrm{H}), 2.34(\mathrm{dd}, J=16.0,5.6 \mathrm{~Hz}, 1 \mathrm{H}), 2.16$ $(\mathrm{dd}, J=16.0,8.2 \mathrm{~Hz}, 1 \mathrm{H}), 2.07-1.80(\mathrm{~m}, 3 \mathrm{H}), 1.70(\mathrm{~s}, 3 \mathrm{H}), 1.60(\mathrm{~s}, 3 \mathrm{H}), 1.27-1.18(\mathrm{~m}, 1 \mathrm{H})$, $1.18-1.08(\mathrm{~m}, 1 \mathrm{H}), 0.79(\mathrm{~d}, J=6.6 \mathrm{~Hz}, 3 \mathrm{H})$.

${ }^{13} \mathrm{C} \mathrm{NMR}\left(126 \mathrm{MHz}, \mathrm{CDCl}_{3}\right): 0208.96,144.10,131.56,128.66,127.88,127.87,126.51,124.43$, 51.17, 49.39, 46.04, 36.97, 28.78, 25.84, 25.52, 19.73, 17.78. 
HRMS: (ESI-TOF) calculated for $\mathrm{C}_{24} \mathrm{H}_{30} \mathrm{NaO}^{+}\left([\mathrm{M}+\mathrm{Na}]^{+}\right): 357.2189$, found: 357.2184 .

FTIR (ATR cm$^{-1}$ ): 2959, 2913, 1709, 1493, 1449, 1407, 1374, 1060, 1031, 747, 696, 627, 609, $547,470$.

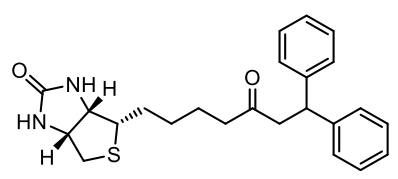

(3aS,4S,6aR)-4-(5-oxo-7,7-diphenylheptyl)tetrahydro-1H-thieno[3,4-d]imidazol-2(3H)-one (21) was prepared according to the general procedure B in DMA and at $0.25 \mathrm{mmol}$ scale and $0.25 \mathrm{M}$ concentration and six equivalents of alkene. The title compound was isolated using automated column chromatography eluting with EtOAc:Hexanes $(60 \% 4 \mathrm{CV}, 60-100 \% 10 \mathrm{CV}$, $100 \% 5 \mathrm{CV}$ ) followed by (35\% MeOH:EtOAc):EtOAc 0-10\% $6 \mathrm{CV}, 10 \% 2 \mathrm{CV}, 15 \% \% 2 \mathrm{CV}, 15-$ $25 \% 5 \mathrm{CV}, 25 \% 12 \mathrm{CV}$ ) to produce a pale yellow oil with $80 \%$ purity. Isolation of authentic product was obtained using supercritical fluid chromatography with a ChiralCel AD-H $(2 \times 25 \mathrm{~cm})$ column and the following conditions: $30 \% \mathrm{EtOH}(0.1 \mathrm{DEA}) / \mathrm{CO}_{2}, 100 \mathrm{bar}, 60 \mathrm{~mL} / \mathrm{min}, 220 \mathrm{~nm}$ to produce a white/yellow semisolid $(28 \mathrm{mg}, 27 \%$ Yield). Comparison of the crude reaction mixture to clean product resulted in $62 \%$ crude NMR yield. When using three equivalents of alkene a $44 \%$ crude NMR yield is obtained.

${ }^{1} \mathrm{H} \mathrm{NMR}\left(500 \mathrm{MHz}, \mathrm{CDCl}_{3}\right): \delta 7.29-7.24(\mathrm{~m}, 4 \mathrm{H}), 7.23-7.20(\mathrm{~m}, 4 \mathrm{H}), 7.17(\mathrm{t}, J=7.2 \mathrm{~Hz}, 2 \mathrm{H})$, $5.66(\mathrm{~s}, 1 \mathrm{H}), 5.26(\mathrm{~s}, 1 \mathrm{H}), 4.59(\mathrm{t}, J=7.6 \mathrm{~Hz}, 1 \mathrm{H}), 4.45(\mathrm{dd}, J=7.8,4.9 \mathrm{~Hz}, 1 \mathrm{H}), 4.22(\mathrm{dd}, J=$ 8.4, 4.6 Hz, 1H), 3.15 (d, $J=7.6 \mathrm{~Hz}, 2 \mathrm{H}$ ), 3.06 (td, $J=7.3,4.5 \mathrm{~Hz}, 1 \mathrm{H}$ ), 2.86 (dd, $J=12.8,4.9$ $\mathrm{Hz}, 1 \mathrm{H}), 2.65(\mathrm{~d}, J=12.8 \mathrm{~Hz}, 1 \mathrm{H}), 2.34(\mathrm{t}, J=7.3 \mathrm{~Hz}, 2 \mathrm{H}), 1.63-1.42(\mathrm{~m}, 4 \mathrm{H}), 1.25(\mathrm{q}, J=7.8$, $7.3 \mathrm{~Hz}, 2 \mathrm{H})$.

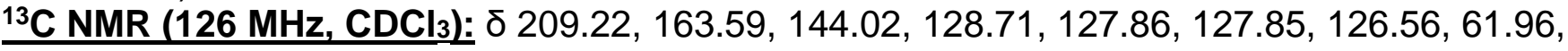
60.18, 55.39, 48.89, 46.11, 43.22, 40.65, 28.38, 28.32, 23.38.

HRMS: (ESI-TOF) calculated for $\mathrm{C}_{24} \mathrm{H}_{28} \mathrm{~N}_{2} \mathrm{NaO}_{2} \mathrm{~S}^{+}\left([\mathrm{M}+\mathrm{Na}]^{+}\right): 431.1764$, found: 431.1770 . FTIR (ATR cm$\left.^{-1}\right): 3234,2925,1701,1493,1451,1371,1330,1265,748,701,608$. 


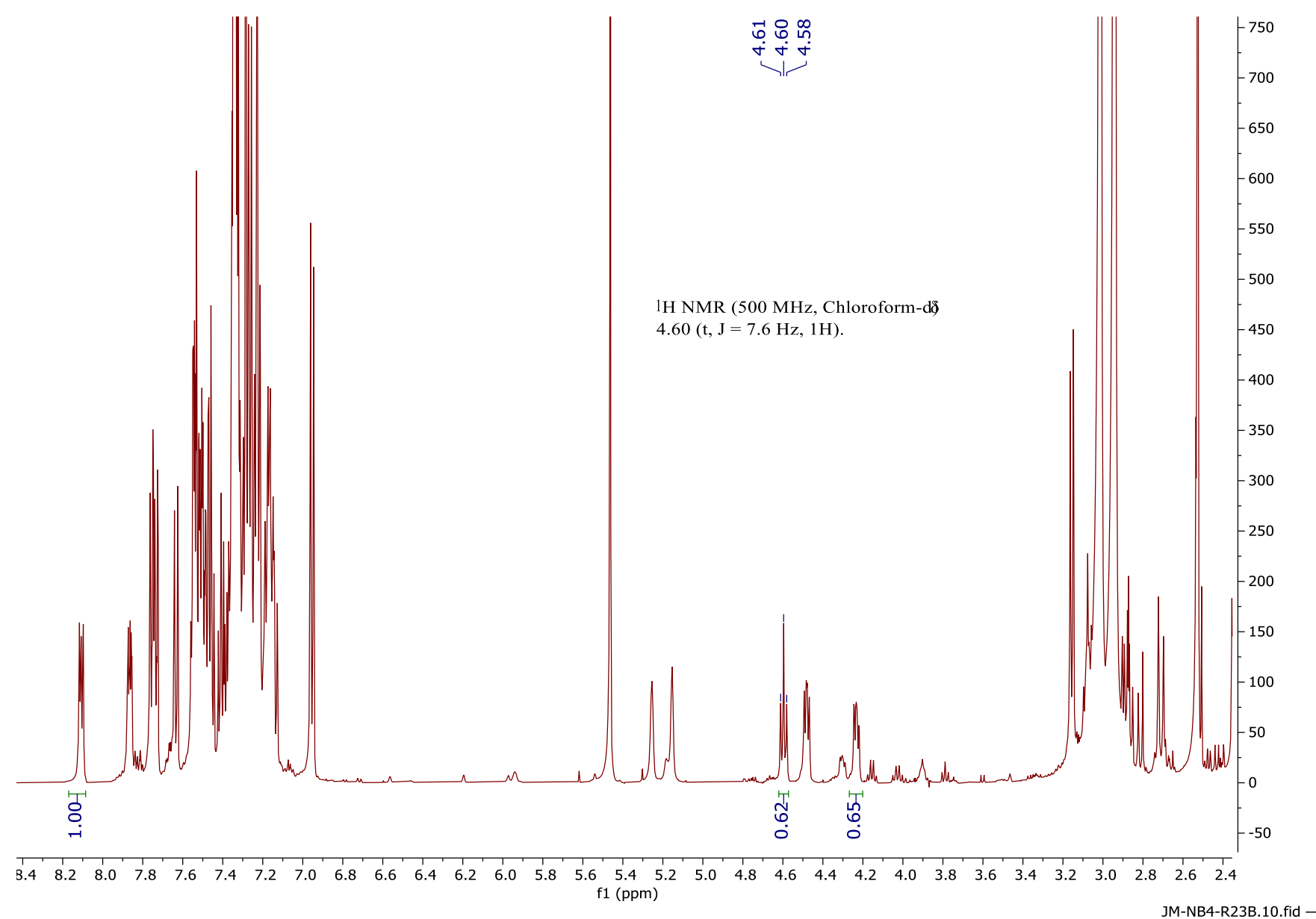

Figure S7. Crude NMR Yield for (21). Referenced to one equivalent of 1-fluoronaphthalene.

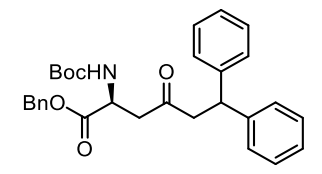

Benzyl (S)-2-((tert-butoxycarbonyl)amino)-4-oxo-6,6-diphenylhexanoate (22) was prepared according to the general procedure $\mathrm{A}$ and at a $0.25 \mathrm{M}$ concentration. The title compound was isolated using automated column chromatography eluting with Ether:Hexanes $(0 \% 4 \mathrm{CV}$, $0-10 \% 4 \mathrm{CV}, 10 \% 6 \mathrm{CV}, 10-25 \% 6 \mathrm{CV}, 25 \% 10 \mathrm{CV}$ ) to produce a slightly yellow tinted white solid (79 $\mathrm{mg}, 32 \%$ Yield).

1H NMR $\left(500 \mathrm{MHz}, \mathrm{CDCl}_{3}\right): \delta 7.34-7.31(\mathrm{~m}, 3 \mathrm{H}), 7.27-7.22(\mathrm{~m}, 6 \mathrm{H}), 7.20-7.14(\mathrm{~m}, 6 \mathrm{H})$, $5.38(\mathrm{~d}, J=8.8 \mathrm{~Hz}, 1 \mathrm{H}), 5.10(\mathrm{~d}, J=12.3 \mathrm{~Hz}, 1 \mathrm{H}), 5.00(\mathrm{~d}, J=12.3 \mathrm{~Hz}, 1 \mathrm{H}), 4.54(\mathrm{t}, J=7.5 \mathrm{~Hz}$, $1 \mathrm{H}), 4.46(\mathrm{dt}, J=8.7,4.4 \mathrm{~Hz}, 1 \mathrm{H}), 3.28-3.04(\mathrm{~m}, 3 \mathrm{H}), 2.86(\mathrm{dd}, J=18.1,4.2 \mathrm{~Hz}, 1 \mathrm{H}), 1.40(\mathrm{~s}$, $9 \mathrm{H})$.

${ }^{13} \mathrm{C} \mathrm{NMR}\left(126 \mathrm{MHz}, \mathrm{CDCl}_{3}\right): \delta 207.07,171.27,155.62,143.65,143.61,135.48,128.78,128.74$, 128.65, 128.44, 128.29, 127.77, 126.70, 126.67, 80.08, 67.38, 49.62, 48.88, 45.89, 45.20, 28.41.

HRMS: (ESI-TOF) calculated for $\mathrm{C}_{30} \mathrm{H}_{34} \mathrm{NO}_{5}{ }^{+}\left([\mathrm{M}+\mathrm{H}]^{+}\right)$: 488.2432 , found: 488.2424 .

FTIR (ATR cm$^{-1}$ ): 1712, 1495, 1453, 1367, 1336, 1264, 1160, 1074, 1025, 731, 697, 607, 493. 


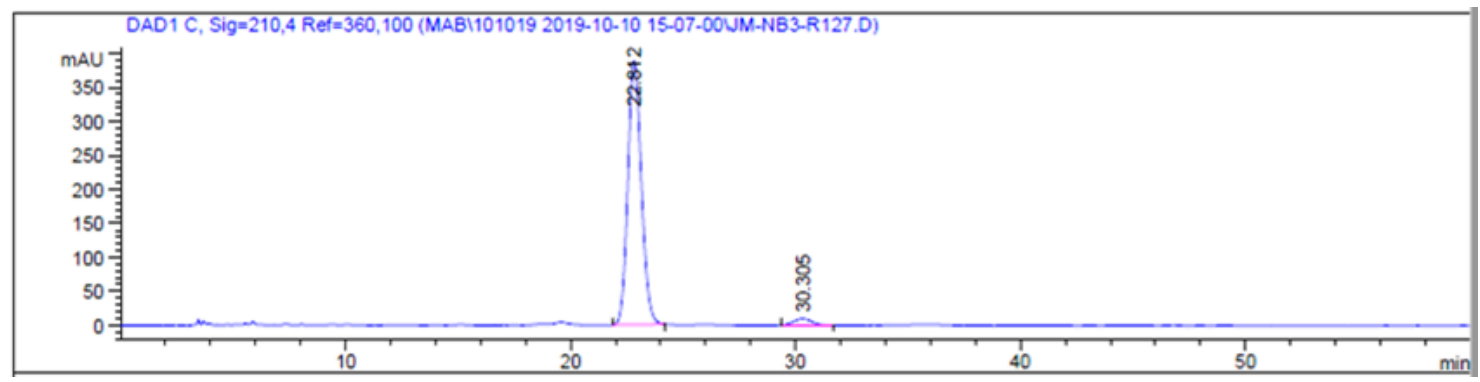

Signal 3: DAD1 C, Sig=210, 4 Ref $=360,100$

\begin{tabular}{|c|c|c|c|c|c|c|}
\hline $\begin{array}{c}\text { Peak } \\
\quad \#\end{array}$ & $\begin{array}{c}\text { RetTime } \\
\text { [min] }\end{array}$ & Type & $\begin{array}{l}\text { Width } \\
\text { [min] }\end{array}$ & $\begin{array}{c}\text { Area } \\
{\left[\mathrm{mAU}^{\star} \mathrm{s}\right]}\end{array}$ & $\begin{array}{l}\text { Height } \\
\text { [mAU] }\end{array}$ & $\begin{array}{c}\text { Area } \\
\text { \& }\end{array}$ \\
\hline- & -- & & & ------- & -------- & $-\cdots--1$ \\
\hline 1 & 22.812 & BB & 0.6448 & $1.62293 \mathrm{e} 4$ & 388.96356 & 96.8081 \\
\hline 2 & 30.305 & BB & 0.7581 & 535.09869 & 9.66287 & 3.1919 \\
\hline
\end{tabular}

Totals: $\quad 1.67644 \mathrm{e} 4 \quad 398.62643$

Figure S8. HPLC trace of benzyl (S)-2-((tert-butoxycarbonyl)amino)-4-oxo-6,6-diphenylhexanoate $(93 \%$ e.e.).

ChiralPak ${ }^{\circledR} \mathrm{IC}, 10 \%$ IPA in Hexanes, 60 min run, $1 \mathrm{~mL} / \mathrm{min}$.

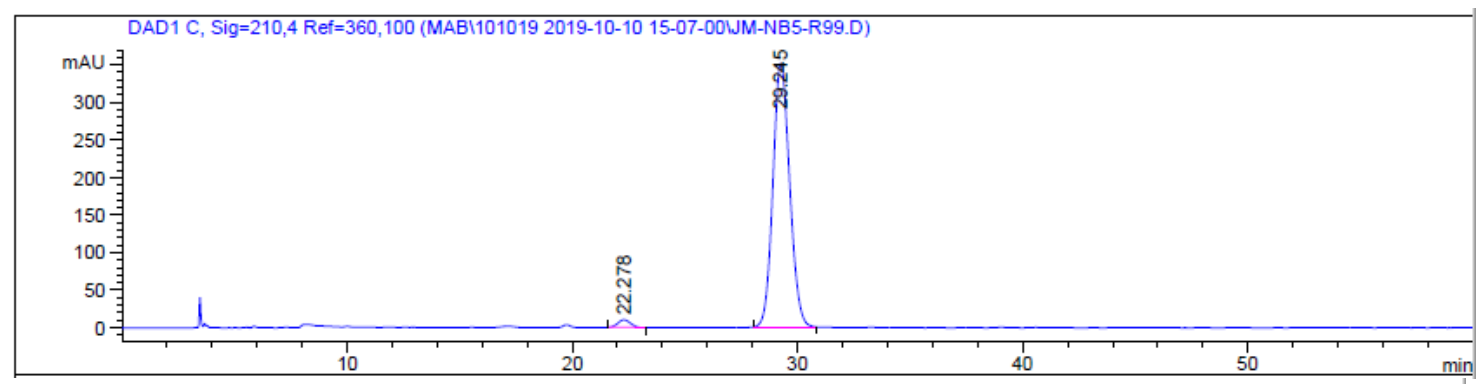

Signal 3: DAD1 C, Sig $=210,4$ Ref $=360,100$

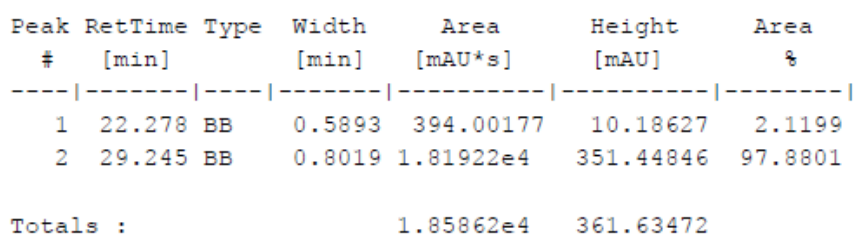

Figure S9. HPLC trace of benzyl (R)-2-((tert-butoxycarbonyl)amino)-4-oxo-6,6-diphenylhexanoate $(95 \%$ e.e.).

ChiralPak ${ }^{\circledR} \mathrm{IC}, 10 \%$ IPA in Hexanes, 60 min run, $1 \mathrm{~mL} / \mathrm{min}$.<smiles>O=C(Cc1cc(Cl)ccc1C(=O)CCc1ccccc1)Nc1ccc(Br)cc1</smiles>

5-(1-(4-bromophenyl)-3-(4-chlorophenyl)-1H-pyrazol-4-yl)-1,1-diphenylpentan-3-one (23) was prepared according to the general Procedure A using 2.4 equivalents of phosphine and at 
a concentration of $0.33 \mathrm{M}$. The title compound was isolated using automated column chromatography eluting with Ether:Hexanes (0\% $5 \mathrm{CV}, 0-10 \% 6 \mathrm{CV}, 10-13 \% 2 \mathrm{CV}, 13-25 \% 4 \mathrm{CV}, 25 \%$ $5 \mathrm{CV}$ ) to produce a white solid $(142.8 \mathrm{mg}, 50 \%$ yield in $91 \%$ purity which corresponds to an isolated yield of $45 \%$ ). Crude NMR analysis shows a $50 \%$ Yield.

${ }^{1} \mathrm{H}$ NMR $\left(500 \mathrm{MHz}, \mathbf{C D C l}_{3}\right): \delta 7.65-7.42(\mathrm{~m}, 6 \mathrm{H}), 7.42-7.34(\mathrm{~m}, 2 \mathrm{H}), 7.29-7.10(\mathrm{~m}, 11 \mathrm{H})$, $4.58(\mathrm{t}, J=7.7 \mathrm{~Hz}, 1 \mathrm{H}), 3.13(\mathrm{~d}, J=7.6 \mathrm{~Hz}, 2 \mathrm{H}), 2.85(\mathrm{t}, J=7.0 \mathrm{~Hz}, 2 \mathrm{H}), 2.63(\mathrm{t}, J=7.0 \mathrm{~Hz}, 2 \mathrm{H})$. ${ }^{13} \mathrm{C} \mathrm{NMR}\left(126 \mathrm{MHz}, \mathrm{CDCl}_{3}\right): \delta 207.93,150.58,143.72,138.98,134.06,132.50,131.91,129.14$, 128.96, 128.74, 127.76, 126.83, 126.68, 120.52, 120.29, 119.50, 48.97, 46.26, 43.81, 18.25 .

HRMS: (ESI-TOF) calculated for $\mathrm{C}_{32} \mathrm{H}_{27} \mathrm{BrClN}_{2} \mathrm{O}^{+}\left([\mathrm{M}+\mathrm{H}]^{+}\right)$: 569.0989 , found: 569.0983 . FTIR (ATR cm$\left.^{-1}\right):$ 1738, 1492, 1450, 1353, 1269, 1155, 1092, 1077, 1009, 984, 955, 902, 874 , 831, 790, 765, 694, 603, 552, 503.

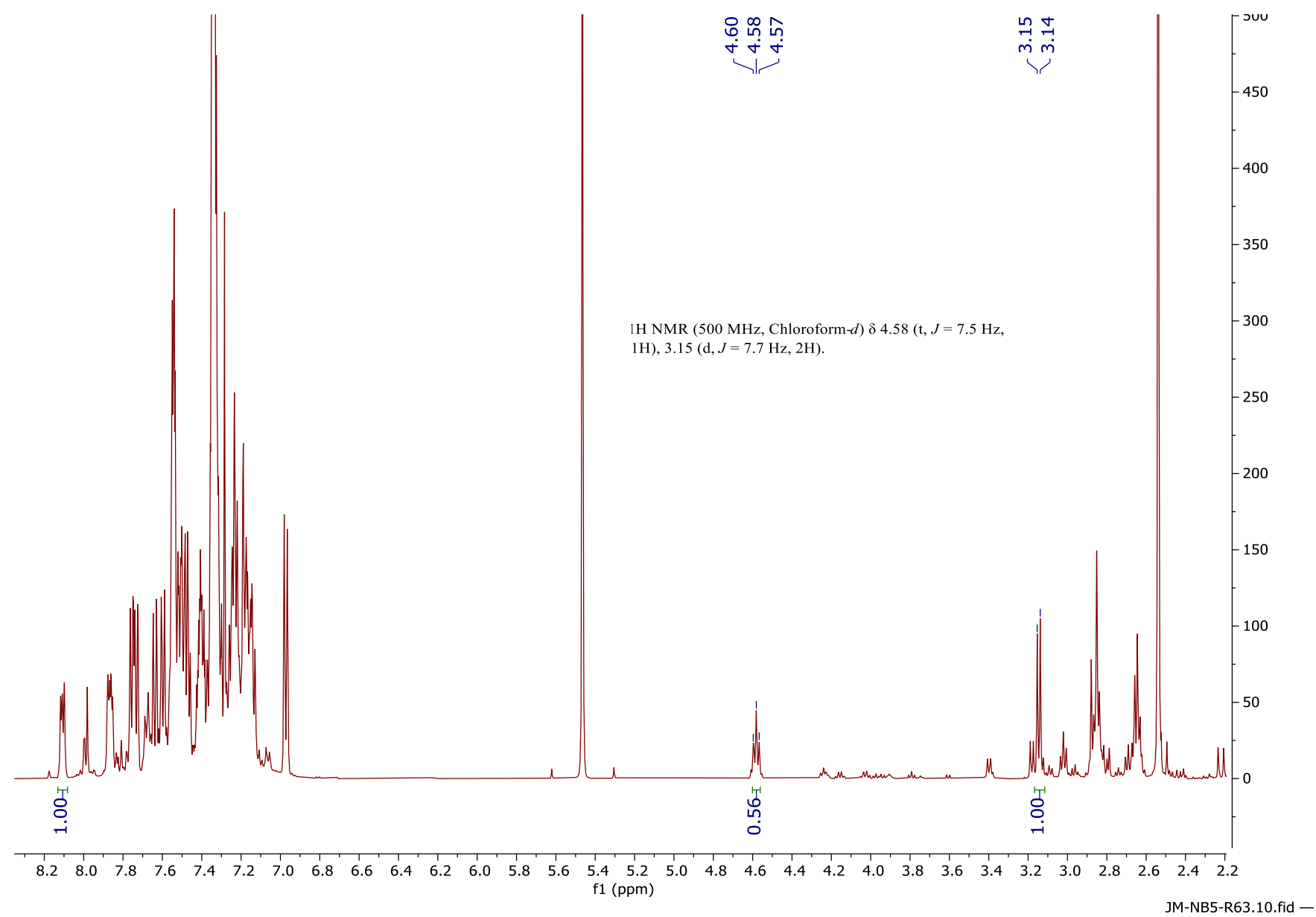

Figure S10. Crude NMR Yield for (23). Referenced to one equivalent of 1-fluoronaphthalene.

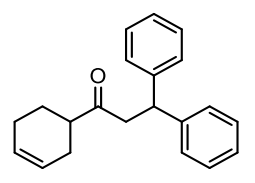

1-(cyclohex-3-en-1-yl)-3,3-diphenylpropan-1-one (24) was prepared according to the general procedure $\mathrm{B}$. The title compound was isolated using automated column chromatography eluting with Ether:Hexanes ( $0 \% 8 \mathrm{CV}, 0-5 \% 12 \mathrm{CV}, 5 \% 10 \mathrm{CV})$ to produce a pale yellow oil $(75 \mathrm{mg}$, $51 \%$ yield in $94 \%$ purity which corresponds to an isolated yield of $48 \%$ ). 
1H NMR (500 MHz, CDCl 3 ): $\delta 7.33-7.23(\mathrm{~m}, 8 \mathrm{H}), 7.22-7.17(\mathrm{~m}, 2 \mathrm{H}), 5.67(\mathrm{~s}, 2 \mathrm{H}), 4.68$ (t, J $=7.5 \mathrm{~Hz}, 1 \mathrm{H}), 3.26(\mathrm{p}, J=9.3 \mathrm{~Hz}, 2 \mathrm{H}), 2.54$ (dddd, $J=11.7,9.1,5.9,2.8 \mathrm{~Hz}, 1 \mathrm{H}), 2.10-1.98$ $(\mathrm{m}, 4 \mathrm{H}), 1.89-1.76(\mathrm{~m}, 1 \mathrm{H}), 1.55-1.44(\mathrm{~m}, 1 \mathrm{H})$.

${ }^{13} \mathrm{C}$ NMR $\left(126 \mathrm{MHz}, \mathrm{CDCl}_{3}\right): \delta 211.43,144.25,144.22,128.66,127.91,127.90,126.75,126.51$, 125.41, 47.22, 47.10, 45.86, 26.66, 24.76, 24.39.

HRMS: (ESI-TOF) calculated for $\mathrm{C}_{21} \mathrm{H}_{22} \mathrm{NaO}^{+}\left([\mathrm{M}+\mathrm{Na}]^{+}\right): 313.1563$, found: 313.1563 .

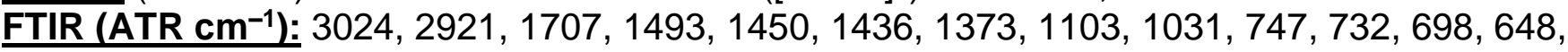
611.

Note: HMBC NMR of (24). Peaks centered at 127.90 and 127.91 are diastereotopic carbons.
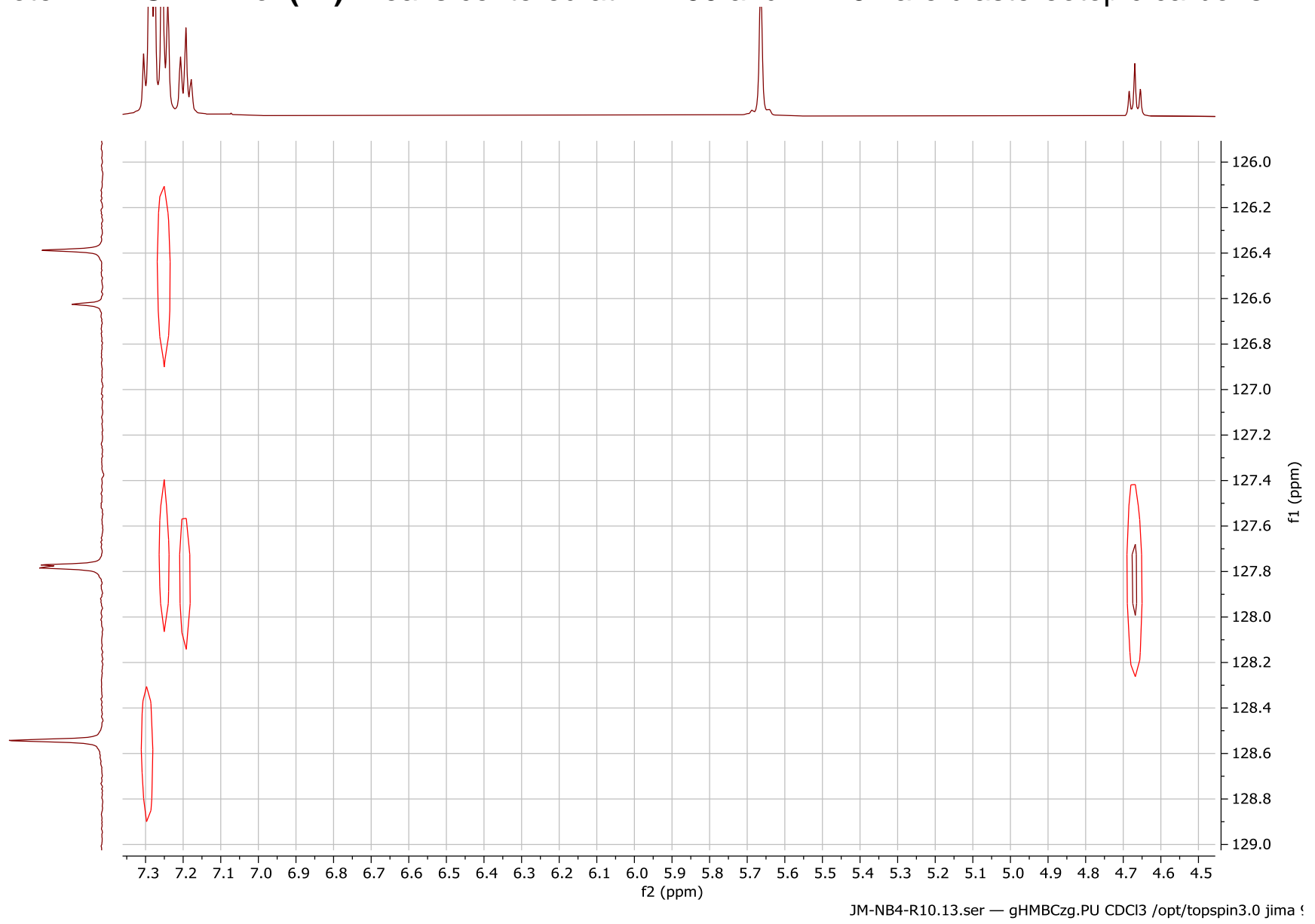<smiles>O=C(CC1CCCCC1)C1(c2ccccc2)CCCC1</smiles>

tert-butyl 4-(3,3-diphenylpropanoyl)piperidine-1-carboxylate (25) was prepared according to the general procedure $\mathrm{A}$. The title compound was isolated using automated column chromatography eluting with Ether:Hexanes (0\% $4 \mathrm{CV}, 0-8 \% 8 \mathrm{CV}, 8 \% 2 \mathrm{CV}, 8-12 \% 2 \mathrm{CV}, 15 \% 15 \mathrm{CV})$ to produce a white solid (157 $\mathrm{mg}, 80 \%$ yield).

${ }^{1} \mathrm{H}$ NMR $\left(500 \mathrm{MHz} \mathrm{CDCl}_{3}\right): \delta 7.30-7.23(\mathrm{~m}, 4 \mathrm{H}), 7.23-7.14(\mathrm{~m}, 6 \mathrm{H}), 4.62(\mathrm{t}, \mathrm{J}=7.4 \mathrm{~Hz}, 1 \mathrm{H})$, $4.01(\mathrm{bs}, 2 \mathrm{H}), 3.20(\mathrm{~d}, \mathrm{~J}=7.5 \mathrm{~Hz}, 2 \mathrm{H}), 2.70(\mathrm{t}, \mathrm{J}=12.2 \mathrm{~Hz}, 2 \mathrm{H}), 2.35(\mathrm{tt}, \mathrm{J}=11.2,3.7 \mathrm{~Hz}, 1 \mathrm{H})$, 1.65 (bs, 2H), $1.43(\mathrm{~s}, 9 \mathrm{H}), 1.42-1.32(\mathrm{~m}, 2 \mathrm{H})$.

${ }^{13} \mathrm{C}$ NMR $\left(126 \mathrm{MHz}, \mathrm{CDCl}_{3}\right): \delta 210.16,154.75,144.03,128.71,127.85,126.61,79.74,49.25$, 46.96, 45.82, 29.85, 28.55, 27.23.

HRMS: (ESI-TOF) calculated for $\mathrm{C}_{25} \mathrm{H}_{31} \mathrm{NNaO}_{3}{ }^{+}\left([\mathrm{M}+\mathrm{Na}]^{+}\right): 416.2196$, found: 416.2200 . 


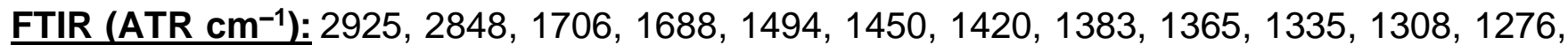
$1248,1232,1159,1129,1079,1064,1028,1014,980,950,921,906,869,791,770,747,702$, $694,650,629,594,555,540,471$.

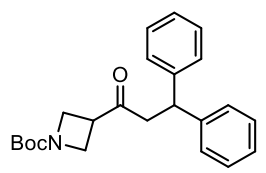

tert-butyl 3-(3,3-diphenylpropanoyl)azetidine-1-carboxylate (26) was prepared according to the general Procedure A using 2.4 equivalents of phosphine and at a concentration of $0.33 \mathrm{M}$. The title compound was isolated using automated column chromatography eluting with Ether:Hexanes ( $0 \% 4 \mathrm{CV}, 0-25 \% 10 \mathrm{CV}, 25 \% 20 \mathrm{CV})$ to produce a slightly yellow tinted white solid (92 mg, $50 \%$ Yield).

${ }^{1} \mathrm{H}$ NMR $\left(500 \mathrm{MHz}, \mathrm{CDCl}_{3}\right): \delta 7.31-7.26(\mathrm{~m}, 4 \mathrm{H}), 7.24-7.12(\mathrm{~m}, 6 \mathrm{H}), 4.61(\mathrm{t}, J=7.5 \mathrm{~Hz}, 1 \mathrm{H})$, $4.00-3.75(\mathrm{~m}, 4 \mathrm{H}), 3.30(\mathrm{p}, J=7.7 \mathrm{~Hz}, 1 \mathrm{H}), 3.17(\mathrm{~d}, J=7.5 \mathrm{~Hz}, 2 \mathrm{H}), 1.40(\mathrm{~s}, 9 \mathrm{H})$.

${ }^{13} \mathrm{C}$ NMR $\left(126 \mathrm{MHz}, \mathbf{C D C l}_{3}\right): \delta 206.32,156.25,143.61,128.84,127.74,126.81,79.91,50.30$, 47.31, 45.95, 39.15, 28.46.

HRMS: (ESI-TOF) calculated for $\mathrm{C}_{23} \mathrm{H}_{27} \mathrm{NNaO}_{3}{ }^{+}\left([\mathrm{M}+\mathrm{Na}]^{+}\right)$: 388.1883 , found: 388.1876 .

FTIR (ATR cm$^{-1}$ ): 2972, 2929, 2887, 1714, 1697, 1598, 1492, 1474, 1448, 1404, 1368, 1342, 1296, 1251, 1163, 1129, 1102, 1083, 1070, 1031, 983, 958, 904, 862, 792, 770, 751, 730, 697, $628,614,587,568,543,494,470,414$.<smiles>O=C(O)CC1(c2ccccc2)C=CC=C1</smiles>

1-cyclopropyl-3,3-diphenylpropan-1-one (27) was prepared according to the general procedure $\mathrm{B}$. The title compound was isolated using automated column chromatography eluting with Ether:Hexanes $(0 \% 8 \mathrm{CV}, 0-5 \% 12 \mathrm{CV}, 5 \% 5 \mathrm{CV})$ to produce a white solid $(88.7 \mathrm{mg}, 70 \%$ yield in $92 \%$ purity which corresponds to an isolated yield of $65 \%)$.

1H NMR (500 MHz, CDCl 3$): \delta 7.35-7.25(\mathrm{~m}, 8 \mathrm{H}), 7.21$ (qd, $J=7.0,6.6,1.7 \mathrm{~Hz}, 2 \mathrm{H}), 4.67(\mathrm{t}, J$ $=7.4 \mathrm{~Hz}, 1 \mathrm{H}), 3.34(\mathrm{dd}, J=7.5,1.7 \mathrm{~Hz}, 2 \mathrm{H}), 1.93(\mathrm{tt}, J=8.2,4.7 \mathrm{~Hz}, 1 \mathrm{H}), 0.96-0.83(\mathrm{~m}, 2 \mathrm{H})$, 0.81 (dd, $J=7.7,3.6 \mathrm{~Hz}, 2 \mathrm{H}$ ).

${ }^{13} \mathrm{C}$ NMR $\left(126 \mathrm{MHz}, \mathrm{CDCl}_{3}\right): \delta$ 208.99, 144.17, 128.64, 127.92, 126.48, 49.72, 46.23, 21.24 , 10.90.

HRMS: (ESI-TOF) calculated for $\mathrm{C}_{18} \mathrm{H}_{19} \mathrm{O}^{+}\left([\mathrm{M}+\mathrm{H}]^{+}\right): 251.1430$, found: 251.1426.

FTIR (ATR cm$^{-1}$ ): 3025, 3003, 2892, 1694, 1596, 1492, 1449, 1412, 1386, 1362, 1244, 1193, 1095, 1070, 1044, 1019, 999, 982, 958, 904, 866, 789, 780, 751, 736, 702, 692, 626, 612, 592, $575,564,548,504,465$.<smiles>O=C(O)CC1(c2ccccc2)CCCC1</smiles>

1-cyclobutyl-3,3-diphenylpropan-1-one (28) was prepared according to the general procedure $\mathrm{B}$. The title compound was isolated using automated column chromatography eluting with Ether:Hexanes ( $0 \% 8 \mathrm{CV}, 0-5 \% 12 \mathrm{CV}, 5 \% 2.5 \mathrm{CV})$ to produce a white solid (99 mg, $74 \%$ yield). 
1H NMR (500 MHz, CDCl$): \delta 7.28-7.24(\mathrm{~m}, 4 \mathrm{H}), 7.23-7.20(\mathrm{~m}, 4 \mathrm{H}), 7.17(\mathrm{td}, J=7.0,1.5 \mathrm{~Hz}$, $2 \mathrm{H}), 4.62(\mathrm{t}, J=7.5 \mathrm{~Hz}, 1 \mathrm{H}), 3.15(\mathrm{p}, J=8.5 \mathrm{~Hz}, 1 \mathrm{H}), 3.09(\mathrm{~d}, J=7.5 \mathrm{~Hz}, 2 \mathrm{H}), 2.10-1.99(\mathrm{~m}$, $4 \mathrm{H}), 1.94-1.83(\mathrm{~m}, 1 \mathrm{H}), 1.75-1.66(\mathrm{~m}, 1 \mathrm{H})$.

${ }^{13} \mathrm{C}$ NMR $\left(126 \mathrm{MHz}, \mathrm{CDCl}_{3}\right)$ : $\delta 209.77,144.24,128.67,127.88,126.51,46.39,46.02,45.87$, 24.11, 17.72.

HRMS: (ESI-TOF) calculated for $\mathrm{C}_{19} \mathrm{H}_{21} \mathrm{O}^{+}\left([\mathrm{M}+\mathrm{H}]^{+}\right): 265.1587$, found: 265.1593 .

FTIR (ATR cm ${ }^{-1}$ ): 2987, 2941, 2860, 1705, 1596, 1493, 1451, 1415, 1376, 1341, 1248, 1120 , 1029, 985, 920, 907, 794, 747, 738, 696, 628, 612, 570, 546, 471.

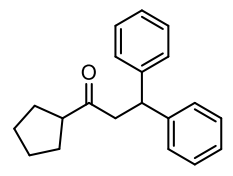

1-cyclopentyl-3,3-diphenylpropan-1-one (29) was prepared according to the general procedure $\mathrm{B}$. The title compound was isolated using automated column chromatography eluting with Ether:Hexanes ( $0 \% 10 \mathrm{CV}, 0-1 \% 3 \mathrm{CV}, 1-5 \% 8 \mathrm{CV}, 5 \% 2 \mathrm{CV})$ to produce a white solid (62 $\mathrm{mg}$, $52 \%$ yield).

1H NMR (500 MHz, CDCl 3 ): $\delta 7.30-7.16(\mathrm{~m}, 8 \mathrm{H}), 7.20-7.13(\mathrm{~m}, 2 \mathrm{H}), 4.64(\mathrm{t}, \mathrm{J}=7.5 \mathrm{~Hz}, 1 \mathrm{H})$, $3.20(\mathrm{~d}, \mathrm{~J}=7.5 \mathrm{~Hz}, 2 \mathrm{H}), 2.82-2.73(\mathrm{~m}, 1 \mathrm{H}), 1.70(\mathrm{dtd}, \mathrm{J}=12.1,8.8,5.3 \mathrm{~Hz}, 2 \mathrm{H}), 1.63-1.46$ $(\mathrm{m}, 6 \mathrm{H})$.

${ }^{13} \mathrm{C} \mathrm{NMR}\left(126 \mathrm{MHz}, \mathrm{CDCl}_{3}\right): \delta 211.09,144.31,128.64,127.92,126.47,52.10,48.14,45.92$, 28.62, 26.02.

HRMS: (ESI-TOF) calculated for $\mathrm{C}_{20} \mathrm{H}_{23} \mathrm{O}^{+}\left([\mathrm{M}+\mathrm{H}]^{+}\right): 279.1743$, found: 279.1750 .

FTIR (ATR cm $\left.{ }^{-1}\right): 2960,1704,1493,1450,1421,1375,1089,1058,1032,743,702,611,568$, $547,471$.<smiles>O=C(c1ccccc1)c1ccccc1</smiles>

1-cyclohexyl-3,3-diphenylpropan-1-one (30) was prepared according to the general procedure $\mathrm{B}$. The title compound was isolated using automated column chromatography eluting with Ether:Hexanes ( $0 \% 8 \mathrm{CV}, 0-5 \% 12 \mathrm{CV}, 5 \% 5 \mathrm{CV})$ to produce a white solid $(87 \mathrm{mg}, 59 \%$ yield). ${ }^{1} \mathrm{H}$ NMR $\left(500 \mathrm{MHz}, \mathrm{CDCl}_{3}\right): \delta 7.28-7.23(\mathrm{~m}, 4 \mathrm{H}), 7.24-7.20(\mathrm{~m}, 4 \mathrm{H}), 7.19-7.14(\mathrm{~m}, 2 \mathrm{H})$, $4.62(\mathrm{t}, J=7.4 \mathrm{~Hz}, 1 \mathrm{H}), 3.19(\mathrm{~d}, J=7.4 \mathrm{~Hz}, 2 \mathrm{H}), 2.28-2.20(\mathrm{~m}, 1 \mathrm{H}), 1.78-1.67(\mathrm{~m}, 4 \mathrm{H}), 1.62$ (d, $J=11.3 \mathrm{~Hz}, 1 \mathrm{H}), 1.27-1.12(\mathrm{~m}, 5 \mathrm{H})$.

${ }^{13} \mathrm{C}$ NMR (126 MHz, $\left.\mathrm{CDCl}_{3}\right): \delta 211.92,144.35,128.63,127.92,126.45,51.45,46.97,45.76$, 28.27, 25.94, 25.73.

HRMS: (ESI-TOF) calculated for $\mathrm{C}_{21} \mathrm{H}_{24} \mathrm{NaO}^{+}\left([\mathrm{M}+\mathrm{Na}]^{+}\right)$: 315.1719 , found: 315.1715 . FTIR (ATR cm ${ }^{-1}$ ): 2928, 2849, 1704, 1494, 1450, 994, 748, 697, 628, 613, 587, 554.

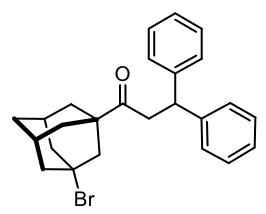

1-((1r,3s,5R,7S)-3-bromoadamantan-1-yl)-3,3-diphenylpropan-1-one (31) was prepared according to the general procedure $\mathrm{B}$. The title compound was isolated using automated column chromatography eluting with a 10\% Ether Hexanes stock solution:Hexanes (0\% $8 \mathrm{CV}, 0-10 \%$ 
$10 \mathrm{CV}, 12 \% 8 \mathrm{CV}, 15 \% 9 \mathrm{CV}, 18 \% 2 \mathrm{CV}, 20 \% 5 \mathrm{CV}$ ) to produce a white solid (100 mg, 47\% yield).

${ }_{1}{ }_{\mathrm{H}} \mathrm{NMR}\left(500 \mathrm{MHz}_{2} \mathbf{C D C l}_{3}\right): \delta 7.30-7.25(\mathrm{~m}, 4 \mathrm{H}), 7.24-7.20(\mathrm{~m}, 4 \mathrm{H}), 7.20-7.15(\mathrm{~m}, 2 \mathrm{H})$, $4.65(\mathrm{t}, J=7.3 \mathrm{~Hz}, 1 \mathrm{H}), 3.20(\mathrm{~d}, J=7.3 \mathrm{~Hz}, 2 \mathrm{H}), 2.34-2.28(\mathrm{~m}, 4 \mathrm{H}), 2.28-2.22(\mathrm{~m}, 2 \mathrm{H}), 2.21$ $-2.17(\mathrm{~m}, 2 \mathrm{H}), 1.76-1.60(\mathrm{~m}, 6 \mathrm{H})$.

${ }^{13} \mathrm{C}$ NMR (126 MHz, $\left.\mathbf{C D C l}_{3}\right): \delta 210.38,144.18,128.65,127.89,126.51,64.23,50.95,48.95$, 48.23, 45.40, 42.84, 36.32, 34.60, 31.78.

HRMS: (ESI-TOF) calculated for $\mathrm{C}_{25} \mathrm{H}_{28} \mathrm{BrO}^{+}\left([\mathrm{M}+\mathrm{H}]^{+}\right): 423.1318$, found: 423.1314 .

FTIR (ATR cm$\left.^{-1}\right):$ 2908, 2855, 1697, 1493, 1449, 1306, 1173, 1153, 1132, 1029, 956, 906, 820, 727, 697, 647, 630, 612, 591, 561, 477.

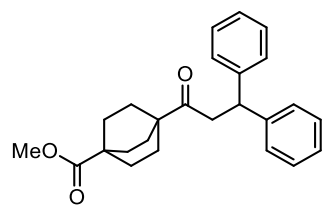

Methyl 4-(3,3-diphenylpropanoyl)bicyclo[2.2.2]octane-1-carboxylate (32) was prepared according to the general procedure $A$. The title compound was isolated using automated column chromatography eluting with Ether:Hexanes (0\% $8 \mathrm{CV}, 0-5 \% 10 \mathrm{CV}, 5 \% 5 \mathrm{CV}, 10 \% 12 \mathrm{CV})$ to produce a white fluffy solid $(50.8 \mathrm{mg}, 54 \%$ yield).

${ }^{1} \mathrm{H}$ NMR $\left(500 \mathbf{M H z} \mathbf{C D C l}_{3}\right): \delta 7.28-7.23(\mathrm{~m}, 4 \mathrm{H}), 7.22-7.13(\mathrm{~m}, 6 \mathrm{H}), 4.63(\mathrm{t}, \mathrm{J}=7.3 \mathrm{~Hz}, 1 \mathrm{H})$, $3.63(\mathrm{~s}, 3 \mathrm{H}), 3.18(\mathrm{~d}, J=7.3 \mathrm{~Hz}, 2 \mathrm{H}), 1.81-1.72(\mathrm{~m}, 6 \mathrm{H}), 1.66-1.60(\mathrm{~m}, 6 \mathrm{H})$.

${ }^{13} \mathrm{C} \mathrm{NMR}\left(126 \mathrm{MHz}_{,} \mathrm{CDCl}_{3}\right): \delta 212.40,177.93,144.34,128.63,127.91,126.46,51.92,45.42$, 44.79, 43.78, 39.03, 27.85, 26.93.

HRMS: (ESI-TOF) calculated for $\mathrm{C}_{25} \mathrm{H}_{29} \mathrm{O}_{3}{ }^{+}\left([\mathrm{M}+\mathrm{H}]^{+}\right): 377.2111$, found: 377.2117 .

FTIR (ATR cm$\left.^{-1}\right)$ : 2947, 2919, 2870, 1720, 1695, 1494,1453, 1432, 1373, 1255, 1237, 1191, 1077, 1061, 1031, 1004, 905, 841, 795, 761, 751, 727, 704, 696, 628, 611, 570, 549, 470.

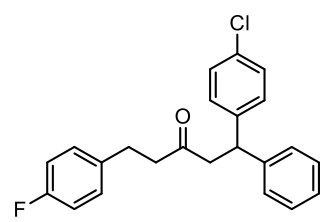

1-(4-chlorophenyl)-5-(4-fluorophenyl)-1-phenylpentan-3-one (33) was prepared according to the general procedure $A$. The title compound was isolated using automated column chromatography eluting with Ether:Hexanes ( $0 \% 8 \mathrm{CV}, 0-5 \% 10 \mathrm{CV}, 5 \% 10 \mathrm{CV})$ to produce a white powdery solid (128 $\mathrm{mg}, 69 \%$ yield).

${ }^{1} \mathrm{H}$ NMR $\left(400 \mathbf{M H z}_{\mathbf{C}} \mathbf{C D C l}_{3}\right): \delta 7.32-7.20(\mathrm{~m}, 5 \mathrm{H}), 7.19-7.10(\mathrm{~m}, 4 \mathrm{H}), 7.06-7.00(\mathrm{~m}, 2 \mathrm{H})$, $6.96-6.89(\mathrm{~m}, 2 \mathrm{H}), 4.58(\mathrm{t}, J=7.5 \mathrm{~Hz}, 1 \mathrm{H}), 3.12(\mathrm{~d}, J=7.5 \mathrm{~Hz}, 2 \mathrm{H}), 2.79(\mathrm{t}, J=7.3 \mathrm{~Hz}, 2 \mathrm{H})$, $2.68-2.60(\mathrm{~m}, 2 \mathrm{H})$.

${ }^{13} \mathrm{C}$ NMR (126 MHz, $\left.\mathbf{C D C l}_{3}\right)$ : $\delta 207.60,161.47$ (d, J = 243.9 Hz), 143.40, 142.43, 136.56 (d, J = $2.9 \mathrm{~Hz}), 132.37,129.81(\mathrm{~d}, \mathrm{~J}=7.8 \mathrm{~Hz}), 129.20,129.19,128.84,127.72,126.82,115.33(\mathrm{~d}, \mathrm{~J}=$ 21.2 Hz), 49.04, 45.41, 45.19, 28.66.

${ }^{19} \mathrm{~F}$ NMR (376 MHz, CDCl 3 ): $\delta-117.20--117.28(\mathrm{~m})$.

HRMS: (ESI-TOF) calculated for $\mathrm{C}_{23} \mathrm{H}_{20} \mathrm{CIFNaO}^{+}\left([\mathrm{M}+\mathrm{Na}]^{+}\right): 389.1079$, found: 389.1078 .

FTIR (ATR $\left.\mathbf{~ c m}^{-1}\right):$ 2925, 1712, 1600, 1508, 1488, 1451, 1407, 1365, 1305, 218, 1156, 1088, 1030, 1013, 858, 821, 750, 717, 697, 594, 556, 533, 478, 420. 


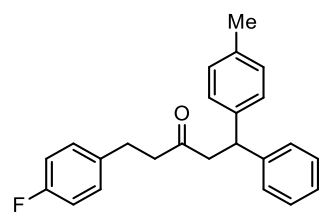

5-(4-fluorophenyl)-1-phfenyl-1-(p-tolyl)pentan-3-one (34) was prepared according to the general procedure $A$. The title compound was isolated using automated column chromatography eluting with Ether:Hexanes ( $0 \% 7 \mathrm{CV}, 0-5 \% 11 \mathrm{CV}, 5 \% 16 \mathrm{CV})$ to produce a slightly yellow tinted solid (126 mg, 73\% yield).

1H NMR (400 MHz, CDCl $\left._{3}\right): \delta 7.28-7.23(\mathrm{~m}, 2 \mathrm{H}), 7.20-7.13(\mathrm{~m}, 3 \mathrm{H}), 7.10-7.05(\mathrm{~m}, 4 \mathrm{H})$, $7.02-6.96(\mathrm{~m}, 2 \mathrm{H}), 6.93-6.85(\mathrm{~m}, 2 \mathrm{H}), 4.54(\mathrm{t}, \mathrm{J}=7.6 \mathrm{~Hz}, 1 \mathrm{H}), 3.11(\mathrm{~d}, \mathrm{~J}=7.6 \mathrm{~Hz}, 2 \mathrm{H}), 2.75$ (t, J = 7.4 Hz, 2H), 2.60 (t, J = 7.6 Hz, 2H), $2.29(\mathrm{~s}, 3 \mathrm{H})$.

${ }^{13}$ C NMR (126 MHz, CDCl 3 ): $\delta$ 208.11, $161.45(\mathrm{~d}, \mathrm{~J}=243.6 \mathrm{~Hz}), 144.13,140.87,136.71(\mathrm{~d}, \mathrm{~J}=$ $3.0 \mathrm{~Hz}), 136.15,129.80(\mathrm{~d}, \mathrm{~J}=7.8 \mathrm{~Hz}), 129.42,128.71,127.76,127.67,126.54,115.28(\mathrm{~d}, \mathrm{~J}=$ $21.2 \mathrm{~Hz}), 49.28,45.84,45.20,28.67,21.11$.

${ }^{19} \mathrm{~F}$ NMR (376 MHz, $\left.\mathrm{CDCl}_{3}\right): \delta-117.31--117.51(\mathrm{~m})$.

HRMS: (ESI-TOF) calculated for $\mathrm{C}_{24} \mathrm{H}_{23} \mathrm{FNaO}^{+}\left([\mathrm{M}+\mathrm{Na}]^{+}\right): 369.1625$, found: 369.1631 .

FTIR (ATR cm$\left.^{-1}\right):$ 1701, 1598, 1508, 1492, 1452, 1417, 1371, 1319, 1258, 1218, 1194, 1157 , 1109, 1078, 1015, 855, 825, 786, 772, 752, 733, 701, 608, 565, 544, 529, 516, 483, 450, 420.

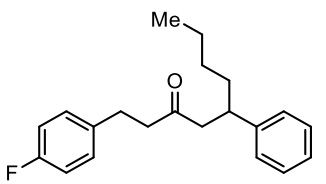

1-(4-fluorophenyl)-5-phenylnonan-3-one (35) was prepared according to the general procedure $\mathrm{A}$. The title compound was isolated using automated column chromatography eluting with Ether:Hexanes $(0 \% 8 \mathrm{CV}, 0-5 \% 12 \mathrm{CV}, 5 \% 5 \mathrm{CV})$ to produce a white oil $(76 \mathrm{mg}, 48 \%$ yield).

${ }^{1} \mathrm{H}$ NMR $\left(500 \mathrm{MHz}, \mathrm{CDCl}_{3}\right): \delta 7.31-7.26(\mathrm{~m}, 2 \mathrm{H}), 7.24-7.17(\mathrm{~m}, 1 \mathrm{H}), 7.16-7.10(\mathrm{~m}, 2 \mathrm{H})$, 7.01 (dd, $J=8.5,5.5 \mathrm{~Hz}, 2 \mathrm{H}), 6.91$ (t, $J=8.7 \mathrm{~Hz}, 2 \mathrm{H}$ ), 3.10 (dt, $J=14.7,7.1 \mathrm{~Hz}, 1 \mathrm{H}$ ), $2.80-$ $2.55(\mathrm{~m}, 5 \mathrm{H}), 2.47(\mathrm{ddd}, J=17.4,8.4,6.7 \mathrm{~Hz}, 1 \mathrm{H}), 1.62-1.48(\mathrm{~m}, 2 \mathrm{H}), 1.35-1.00(\mathrm{~m}, 4 \mathrm{H})$, $0.81(\mathrm{t}, \mathrm{J}=7.3 \mathrm{~Hz}, 3 \mathrm{H})$.

${ }^{13} \mathrm{C}$ NMR (126 MHz, CDCl 3 ): $\delta$ 209.10, $161.42(\mathrm{~d}, \mathrm{~J}=243.7 \mathrm{~Hz}), 144.65,136.80(\mathrm{~d}, \mathrm{~J}=3.3 \mathrm{~Hz})$, $129.79(\mathrm{~d}, \mathrm{~J}=7.8 \mathrm{~Hz}), 128.60,127.60,126.46,115.26(\mathrm{~d}, \mathrm{~J}=21.1 \mathrm{~Hz}), 50.47,45.24(\mathrm{~d}, \mathrm{~J}=1.1$ $\mathrm{Hz}), 41.50,36.26,29.70,28.72,22.72,14.09$.

${ }^{19} \mathrm{~F}$ NMR (376 MHz, CDCl 3 ): $\delta-118.33(\mathrm{tt}, \mathrm{J}=9.0,4.6 \mathrm{~Hz})$.

HRMS: (ESI-TOF) calculated for $\mathrm{C}_{21} \mathrm{H}_{25} \mathrm{FNaO}^{+}\left([\mathrm{M}+\mathrm{Na}]^{+}\right): 335.1782$, found: 335.1786 .

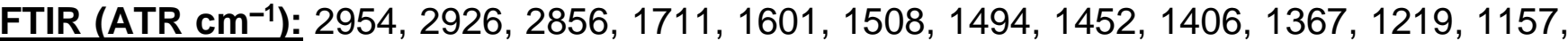
1095, 1073, 823, 757, 727, 699, 533, 479, 420.

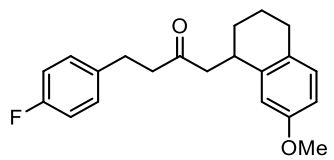

4-(4-fluorophenyl)-1-(7-methoxy-1,2,3,4-tetrahydronaphthalen-1-yl)butan-2-one (36) was prepared according to the general procedure $A$. The title compound was isolated using automated column chromatography eluting with Ether:Hexanes (0\% $8 \mathrm{CV}, 0-5 \% 10 \mathrm{CV}, 5 \% 18 \mathrm{CV}$, $8 \% 2 \mathrm{CV}$ ) to produce a yellow tinted white solid (110 mg, $66 \%$ yield).

${ }^{1} \mathrm{H}$ NMR $\left(500 \mathrm{MHz} \mathrm{CDCl}_{3}\right): \delta 7.14(\mathrm{dd}, J=8.3,5.5 \mathrm{~Hz}, 2 \mathrm{H}), 6.96(\mathrm{td}, J=8.5,1.8 \mathrm{~Hz}, 3 \mathrm{H}), 6.68$ (dd, $J=8.4,2.7 \mathrm{~Hz}, 1 \mathrm{H}), 6.60-6.57(\mathrm{~m}, 1 \mathrm{H}), 3.74(\mathrm{~s}, 3 \mathrm{H}), 3.38(\mathrm{dq}, J=10.5,5.6 \mathrm{~Hz}, 1 \mathrm{H}), 2.89$ 
(t, $J=7.4 \mathrm{~Hz}, 2 \mathrm{H}), 2.78-2.60(\mathrm{~m}, 6 \mathrm{H}), 1.87-1.79(\mathrm{~m}, 1 \mathrm{H}), 1.75-1.68(\mathrm{~m}, 2 \mathrm{H}), 1.56-1.46$ $(\mathrm{m}, 1 \mathrm{H})$.

${ }^{13} \mathrm{C} \mathrm{NMR}\left(126 \mathrm{MHz}, \mathrm{CDCl}_{3}\right): \delta 209.16,161.52(\mathrm{~d}, \mathrm{~J}=243.9 \mathrm{~Hz}), 157.76,140.97,136.74(\mathrm{~d}, J=$ $3.3 \mathrm{~Hz}), 130.25,129.90(\mathrm{~d}, J=7.8 \mathrm{~Hz}), 129.35,115.38(\mathrm{~d}, J=21.1 \mathrm{~Hz}), 113.22,112.21,55.39$, $50.89,45.21,33.52,29.09,28.81,28.43,19.91$.

${ }^{19} \mathrm{~F} \mathrm{NMR}\left(376 \mathrm{MHz} \mathrm{CDCl}_{3}\right): \delta-117.23$ (tt, $\left.J=9.0,4.9 \mathrm{~Hz}\right)$.

HRMS: (ESI-TOF) calculated for $\mathrm{C}_{21} \mathrm{H}_{23} \mathrm{FNaO}_{2}{ }^{+}\left([\mathrm{M}+\mathrm{Na}]^{+}\right): 349.1574$, found: 349.1573 .

FTIR (ATR cm$^{-1}$ ): 2927, 1709, 1607, 1507, 1463, 1450, 1359, 1278, 1247, 1217, 1156, 1125, 1096, 1038, 1015, 809, 701, 533, 477.

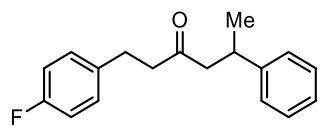

1-(4-fluorophenyl)-5-phenylhexan-3-one (37) was prepared according to the general procedure $\mathrm{A}$. The title compound was isolated using automated column chromatography eluting with Ether:Hexanes ( $0 \% 4 \mathrm{CV}, 0-10 \% 10 \mathrm{CV}, 10 \% 12 \mathrm{CV})$ to produce a yellow white solid $(97 \mathrm{mg}$, $71 \%$ yield).

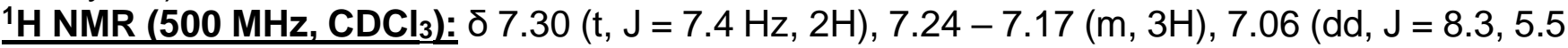
$\mathrm{Hz}, 2 \mathrm{H}), 6.94(\mathrm{t}, \mathrm{J}=8.5 \mathrm{~Hz}, 2 \mathrm{H}), 3.32(\mathrm{sx}, \mathrm{J}=7.1 \mathrm{~Hz}, 1 \mathrm{H}), 2.85-2.70(\mathrm{~m}, 3 \mathrm{H}), 2.69-2.51(\mathrm{~m}$, $3 \mathrm{H}), 1.26(\mathrm{~d}, \mathrm{~J}=6.8 \mathrm{~Hz}, 3 \mathrm{H})$.

${ }^{13} \mathrm{C}$ NMR (126 MHz, CDCl 3 ): $\delta 208.83,161.42(\mathrm{~d}, J=243.7 \mathrm{~Hz}), 146.15,136.76(\mathrm{~d}, J=3.2 \mathrm{~Hz})$, 129.79 (d, $J=7.8 \mathrm{~Hz}), 128.66,126.87,126.45,115.27$ (d, $J=21.1 \mathrm{~Hz}), 51.48,45.11$ (d, $J=1.1$ $\mathrm{Hz}), 35.62,28.77,22.09$.

${ }^{19} \mathrm{~F}$ NMR (376 MHz, $\left.\mathrm{CDCl}_{3}\right): \delta-117.33--117.46(\mathrm{~m})$.

HRMS: (ESI-TOF) calculated for $\mathrm{C}_{18} \mathrm{H}_{20} \mathrm{FO}^{+}\left([\mathrm{M}+\mathrm{H}]^{+}\right): 271.1493$, found: 271.1491.

FTIR (ATR cm$^{-1}$ ): 1601, 1508, 1493, 1451, 1407, 1365, 1218, 1157, 1113, 1095, 1074, 1015, $822,760,698,532,478,422$.

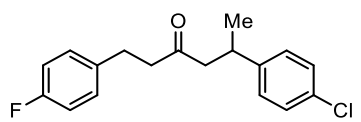

5-(4-chlorophenyl)-1-(4-fluorophenyl)hexan-3-one (38) was prepared according to the general procedure $A$. The title compound was isolated using automated column chromatography eluting with Ether:Hexanes (0\% $8 \mathrm{CV}, 0-5 \% 12 \mathrm{CV}, 5-10 \% 2 \mathrm{CV}, 10 \% 5 \mathrm{CV})$ to produce a white solid (Run $1=93.9 \mathrm{mg}, 61 \%$ yield).

${ }^{1} \mathrm{H}$ NMR $\left(500 \mathrm{MHz} \mathbf{C D C l}_{3}\right): \delta 7.30-7.25(\mathrm{~m}, 2 \mathrm{H}), 7.16-7.11(\mathrm{~m}, 2 \mathrm{H}), 7.10-7.04(\mathrm{~m}, 2 \mathrm{H})$, $7.00-6.90(\mathrm{~m}, 2 \mathrm{H}), 3.32(\mathrm{~h}, J=7.1 \mathrm{~Hz}, 1 \mathrm{H}), 2.82(\mathrm{t}, J=7.8 \mathrm{~Hz}, 2 \mathrm{H}), 2.74-2.52(\mathrm{~m}, 4 \mathrm{H}), 1.24$ (d, $J=7.1 \mathrm{~Hz}, 3 \mathrm{H})$.

${ }^{13} \mathrm{C} \mathrm{NMR}\left(126 \mathrm{MHz}, \mathrm{CDCl}_{3}\right): \delta 208.45,161.47(\mathrm{~d}, J=243.8 \mathrm{~Hz}), 144.64,136.66$ (d, $\left.J=3.3 \mathrm{~Hz}\right)$, 132.06, $129.81(\mathrm{~d}, J=7.9 \mathrm{~Hz}), 128.76,128.30,115.32(\mathrm{~d}, J=21.2 \mathrm{~Hz}), 51.33,45.15,34.94$, 28.76, 22.08.

${ }^{19} \mathrm{~F}$ NMR (376 MHz, $\left.\mathrm{CDCl}_{3}\right): \delta-117.17--117.30(\mathrm{~m})$.

HRMS: (ESI-TOF) calculated for $\mathrm{C}_{18} \mathrm{H}_{18} \mathrm{CIFNaO}^{+}\left([\mathrm{M}+\mathrm{Na}]^{+}\right)$: 327.0923 , found: 327.0926 .

FTIR (ATR cm$^{-1}$ ): 2925, 1712, 1600, 1508, 1488, 1451, 1407, 1365, 1305, 1218, 1156, 1088 , 1030, 1013, 858, 821, 750, 717, 697, 594, 556, 533, 478, 420.

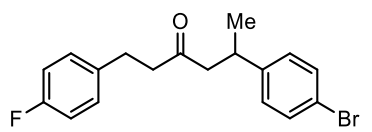


5-(4-bromophenyl)-1-(4-fluorophenyl)hexan-3-one (39) was prepared according to the general procedure $\mathrm{A}$. The title compound was isolated using automated column chromatography eluting with Ether:Hexanes ( $0 \% 8 \mathrm{CV}, 0-5 \% 10 \mathrm{CV}, 5 \% 5 \mathrm{CV}, 10 \% 7 \mathrm{CV})$ to produce a white solid (Run $1=97.9 \mathrm{mg}, 56 \%$ yield).

${ }^{1} \mathrm{H}$ NMR $\left(500 \mathrm{MHz} \mathrm{CDCl}_{3}\right): \delta 7.42-7.35(\mathrm{~m}, 2 \mathrm{H}), 7.05(\mathrm{dt}, J=8.4,2.7 \mathrm{~Hz}, 4 \mathrm{H}), 6.93(\mathrm{t}, J=8.7$ $\mathrm{Hz}, 2 \mathrm{H}), 3.27(\mathrm{~h}, J=7.0 \mathrm{~Hz}, 1 \mathrm{H}), 2.79(\mathrm{t}, J=7.8 \mathrm{~Hz}, 2 \mathrm{H}), 2.70-2.50(\mathrm{~m}, 4 \mathrm{H}), 1.21(\mathrm{~d}, J=7.0$ $\mathrm{Hz}, 3 \mathrm{H})$.

${ }^{13} \mathrm{C} \mathrm{NMR}\left(126 \mathrm{MHz}, \mathrm{CDCl}_{3}\right): \delta 208.42,161.48(\mathrm{~d}, J=243.9 \mathrm{~Hz}), 145.17,136.66$ (d, $\left.J=3.2 \mathrm{~Hz}\right)$, $131.72,129.82(\mathrm{~d}, J=7.8 \mathrm{~Hz}), 128.72,120.12,115.34(\mathrm{~d}, J=21.1 \mathrm{~Hz}), 51.27,45.16(\mathrm{~d}, J=1.1$ $\mathrm{Hz}), 35.01,28.78,22.03$.

${ }^{19} \mathrm{~F}$ NMR $\left(376 \mathrm{MHz}, \mathrm{CDCl}_{3}\right): \delta-117.23(\mathrm{tt}, \mathrm{J}=8.4,5.3 \mathrm{~Hz})$.

HRMS: (ESI-TOF) calculated for $\mathrm{C}_{18} \mathrm{H}_{18} \mathrm{BrFNaO}^{+}\left([\mathrm{M}+\mathrm{Na}]^{+}\right): 371.0417$, found: 371.0420 .

FTIR (ATR m$\left.^{-1}\right): 3027,2925,1712,1600,1508,1488,1451,1407,1365,1305,1218,1156$, $1088,1030,1013,858,821,750,717,697,594,556,533,478,420$.

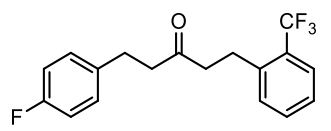

1-(4-fluorophenyl)-5-(2-(trifluoromethyl)phenyl)pentan-3-one (40) was prepared according to the general procedure $\mathrm{A}$. The title compound was isolated using automated column chromatography eluting with Ether:Hexanes ( $0 \% 4 \mathrm{CV}, 0-10 \% 10 \mathrm{CV}, 10 \% 10 \mathrm{CV})$ to produce a yellow white solid (Run $1=87 \mathrm{mg}, 53 \%$ yield).

${ }^{1} \mathrm{H}$ NMR $\left(500 \mathrm{MHz}, \mathrm{CDCl}_{3}\right): \delta 7.59(\mathrm{~d}, J=7.9 \mathrm{~Hz}, 1 \mathrm{H}), 7.42(\mathrm{t}, J=7.6 \mathrm{~Hz}, 1 \mathrm{H}), 7.31-7.23(\mathrm{~m}$, $2 \mathrm{H}), 7.13-7.06(\mathrm{~m}, 2 \mathrm{H}), 6.93(\mathrm{t}, J=8.6 \mathrm{~Hz}, 2 \mathrm{H}), 3.03(\mathrm{t}, J=7.8 \mathrm{~Hz}, 2 \mathrm{H}), 2.86(\mathrm{t}, J=7.5 \mathrm{~Hz}$, $2 \mathrm{H}), 2.70-2.64(\mathrm{~m}, 4 \mathrm{H})$.

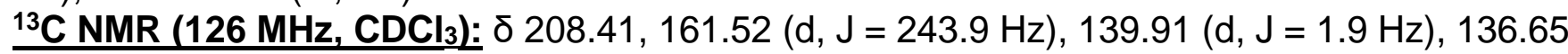
$(\mathrm{d}, \mathrm{J}=3.2 \mathrm{~Hz}), 132.07,131.30,129.86(\mathrm{~d}, \mathrm{~J}=7.8 \mathrm{~Hz}), 128.58(\mathrm{q}, \mathrm{J}=29.8 \mathrm{~Hz}), 126.46,126.23$ (q, J = 5.7 Hz), $124.68(q, J=273.7 \mathrm{~Hz}), 115.38(d, J=21.1 \mathrm{~Hz}), 44.76,44.44,29.05,26.68$ (d, $\mathrm{J}=1.9 \mathrm{~Hz})$.

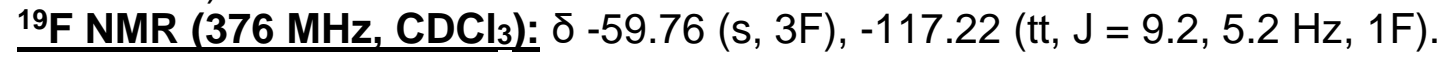

HRMS: (ESI-TOF) calculated for $\mathrm{C}_{18} \mathrm{H}_{17} \mathrm{~F}_{4} \mathrm{O}^{+}\left([\mathrm{M}+\mathrm{H}]^{+}\right): 325.1210$, found: 325.1213 .

FTIR (ATR cm$\left.^{-1}\right):$ 2931, 1714, 1607, 1583, 1509, 1453, 1415, 1372, 1311, 1220, 1158, 1113, $1059,1037,1016,979,956,909,823,767,731,651,598,533,478,425$

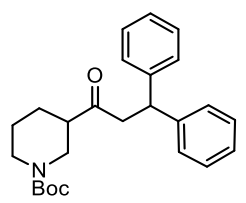

tert-butyl 3-(3,3-diphenylpropanoyl)piperidine-1-carboxylate (41) was prepared according to the general procedure $A$. The title compound was isolated using automated column chromatography eluting with Ether:Hexanes $(0 \% 6 \mathrm{CV}, 0-20 \% 12 \mathrm{CV}, 20 \% 8 \mathrm{CV})$ to produce a white solid ( $140 \mathrm{mg}, 71 \%$ yield).

${ }^{1} \mathrm{H}$ NMR $\left(500 \mathrm{MHz}_{2} \mathrm{CDCl}_{3}\right): \delta 7.31-7.24(\mathrm{~m}, 5 \mathrm{H}), 7.24-7.20(\mathrm{~m}, 3 \mathrm{H}), 7.17(\mathrm{t}, \mathrm{J}=7.3 \mathrm{~Hz}, 2 \mathrm{H})$, $4.61(\mathrm{t}, \mathrm{J}=7.4 \mathrm{~Hz}, 1 \mathrm{H}), 3.90$ (bs, 2H), $3.31-3.17(\mathrm{~m}, 2 \mathrm{H}), 2.79(\mathrm{bs}, 1 \mathrm{H}), 2.70(\mathrm{bs}, 1 \mathrm{H}), 2.41$ (bs, $1 \mathrm{H}), 1.81$ (bs, 1H), 1.63 (bs, $1 \mathrm{H}), 1.44(\mathrm{~s}, 9 \mathrm{H}), 1.42-1.34(\mathrm{~m}, 2 \mathrm{H})$.

${ }^{13} \mathrm{C}$ NMR $\left(126 \mathrm{MHz}, \mathrm{CDCl}_{3}\right): \delta 209.45,154.80,143.98,128.72,128.70,127.87,127.81,126.58$, 79.87, 49.15, 47.56, 45.68, 28.56, 26.67, 24.51.

HRMS: (ESI-TOF) calculated for $\mathrm{C}_{25} \mathrm{H}_{31} \mathrm{NNaO}_{3}{ }^{+}\left([\mathrm{M}+\mathrm{Na}]^{+}\right): 416.2196$, found: 416.2192 . 
FTIR (ATR cm$^{-1}$ ): 2932, 2861,1697, 1653,1495, 1473, 1463, 1451, 1417, 1364, 1348, 1301, 1290, 1266, 1237, 1106, 1090, 1079, 1060, 1043, 1033, 1015, 996, 960, 940, 918, 889, 866, $854,791,762,742$.

\section{Scale Up}

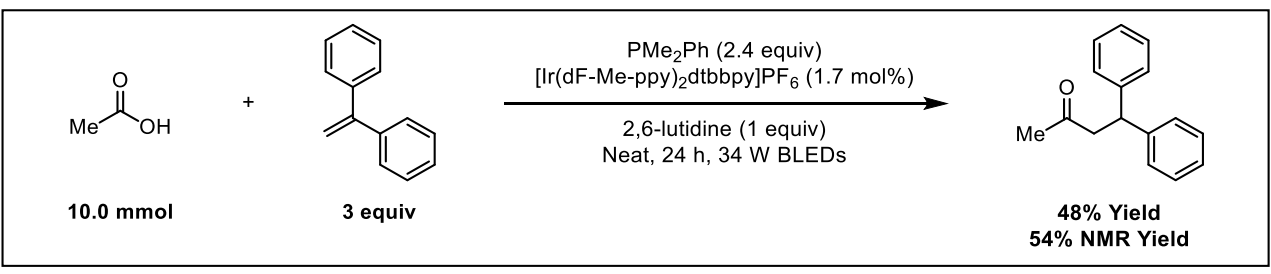

An oven-dried $20 \mathrm{~mL}$ reaction vial (Fisher® glass vials, 03-337-15) was charged with Iridium (172 mg, $0.017 \mathrm{mmol}, 0.017$ equiv) and equipped with a PTFE-coated stir bar. The vial was Teflon taped on the threads, and then taken into a $\mathrm{N}_{2}$-filled glovebox. To the vial was added diphenyl ethylene (5.30 mL, $30 \mathrm{mmol}, 3.0$ equiv), 2,6-lutidine (1.16 mL, $10 \mathrm{mmol}, 1.0$ equiv), and acetic acid ( $571 \mu \mathrm{L}, 10.0 \mathrm{mmol}, 1.0$ equiv). Finally, phosphine ( $3.41 \mathrm{~mL}, 24 \mathrm{mmol}, 2.4$ equiv) was added. The vial was then capped and sealed with electrical tape. The vial was irradiated for $24 \mathrm{~h}$ in a Photoreactor (800 rpm, 1500 fan speed, 100\% light intensity). An aliquot of the crude reaction mixture was analzed by ${ }^{1} \mathrm{H}-\mathrm{NMR}$ with 1 -fluoronaphthalene $(647 \mu \mathrm{L}, 5.0 \mathrm{mmol}$, 0.5 equiv) as an external standard. Comparison of the crude reaction mixture to clean product resulted in a 54\% crude NMR Yield. The title compound, 4,4-diphenylbutan-2-one (19) was isolated using automated column chromatography eluting with Ether:Hexanes $(0 \% 15 \mathrm{CV}, 0-8 \%$ $12 \mathrm{CV}, 15 \% 3 \mathrm{CV}$ ) to produce a yellow oil that solidified into a white solid inside the freezer (1.09 $\mathrm{g}, 48 \%$ yield).

\section{Mechanistic Studies}

In the course of optimization, differential reactivity was observed between electron neutral and electron deficient alkenes in the presence of different phosphines. To probe the origin of this reactivity, emission quenching experiments were undertaken to rule out the possibility of competitive electron transfer to the alkenes.

Table S10. Reactivity observed with diphenyl ethylene, alpha methyl styrene, and 1-(trifluoromethyl)-2-vinylbenzene in the coupling of 3-(4-fluorophenyl)propanoic acid with triphenyl phosphine, ethyl diphenyl phosphinite, and dimethyl phenyl phosphine

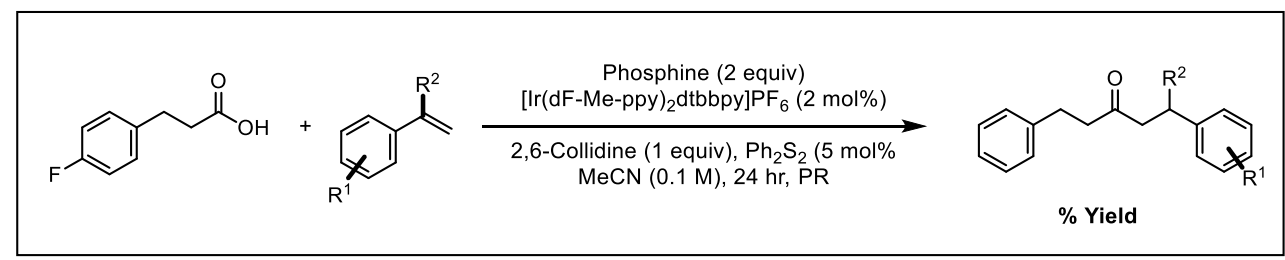




\begin{tabular}{lllll}
\hline Entry & \% Yield $\left(\mathrm{POEtPh}_{2}\right)$ & \% Yield $\left(\mathrm{PPh}_{3}\right)$ & \% Yield $\left(\mathrm{PMe}_{2} \mathrm{Ph}\right)$ \\
\hline & 26 & 6 & 81 \\
\hline
\end{tabular}

aYield was determined by ${ }^{1} \mathrm{H}$ NMR spectroscopy using 1-fluoronaphthalene as an external standard.

\section{A. Emission Quenching Experiments}

Absorption and Emission experiments were conducted in line with our previous publication on the arylation of ethereal $\mathrm{C}-\mathrm{H}$ bonds. ${ }^{7}$ An excitation wavelength of $415 \mathrm{~nm}$ and an emission wavelength of $515 \mathrm{~nm}$ were used for monitoring quenching of the iridium photocatalyst. All reagents were prepared in stock solutions inside a nitrogen filled glove box. Reagents were diluted in acetonitrile $(3 \mathrm{~mL})$ and sealed in a screw-top $1.0 \mathrm{~cm}$ quartz cuvette. A blank composed of acetonitrile was used in absorbance measurements. Samples for quenching experiment were dispensed from a stock solution of $\operatorname{Ir}\left[\mathrm{dF}(\mathrm{Me}) \mathrm{ppy}_{2}(\mathrm{dtbbpy}) \mathrm{PF}_{6}(1.314 \mathrm{mM}\right.$ in $\mathrm{MeCN}$, amount dispensed: $456 \mu \mathrm{L}, 0.6 \mu \mathrm{mol}, 2.0 \times 10^{-4} \mathrm{M}$ after dilution) followed by addition of quenchers that were also prepared from stock solutions in $\mathrm{MeCN}$. Absorption spectra were collected on an Agilent Technologies Cary 60 UV-Vis Spectrophotometer. Emission quenching data were collected on an Agilent Cary Eclipse Fluorescence Spectrophotometer with excitation and emissions slit widths of 2.5 and $5 \mathrm{~nm}$ were used, respectively.
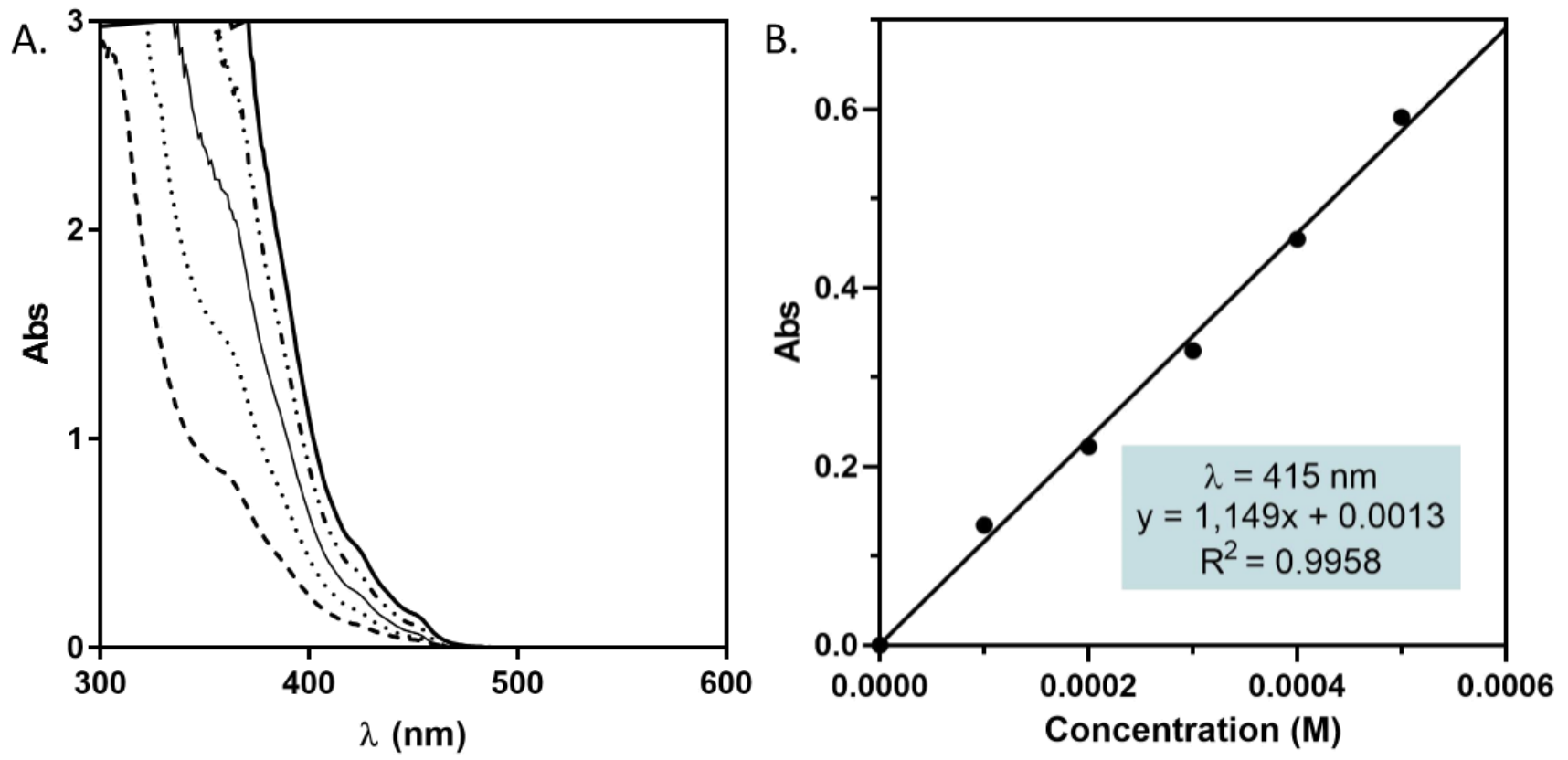

Figure S11. A. The electronic absorption spectra of $\operatorname{Ir}[\mathrm{dF}(\mathrm{Me}) \mathrm{ppy}]_{2}(\mathrm{dtbbpy}) \mathrm{PF} 6$ at concentrations ranging from $1.0 \times 10^{-4}$ to $5.0 \times 10^{-4} \mathrm{M}$ in MeCN. On the right is the calibration curve for $\operatorname{lr}\left[\mathrm{dF}(\mathrm{Me}) \mathrm{ppy}_{2}(\mathrm{dtbbpy}) \mathrm{PF} 6\right.$ at a wavelength of $415 \mathrm{~nm}\left(\varepsilon=1.149 \times 10^{3} \mathrm{M}^{-1} \mathrm{~cm}^{-1}\right)$. 


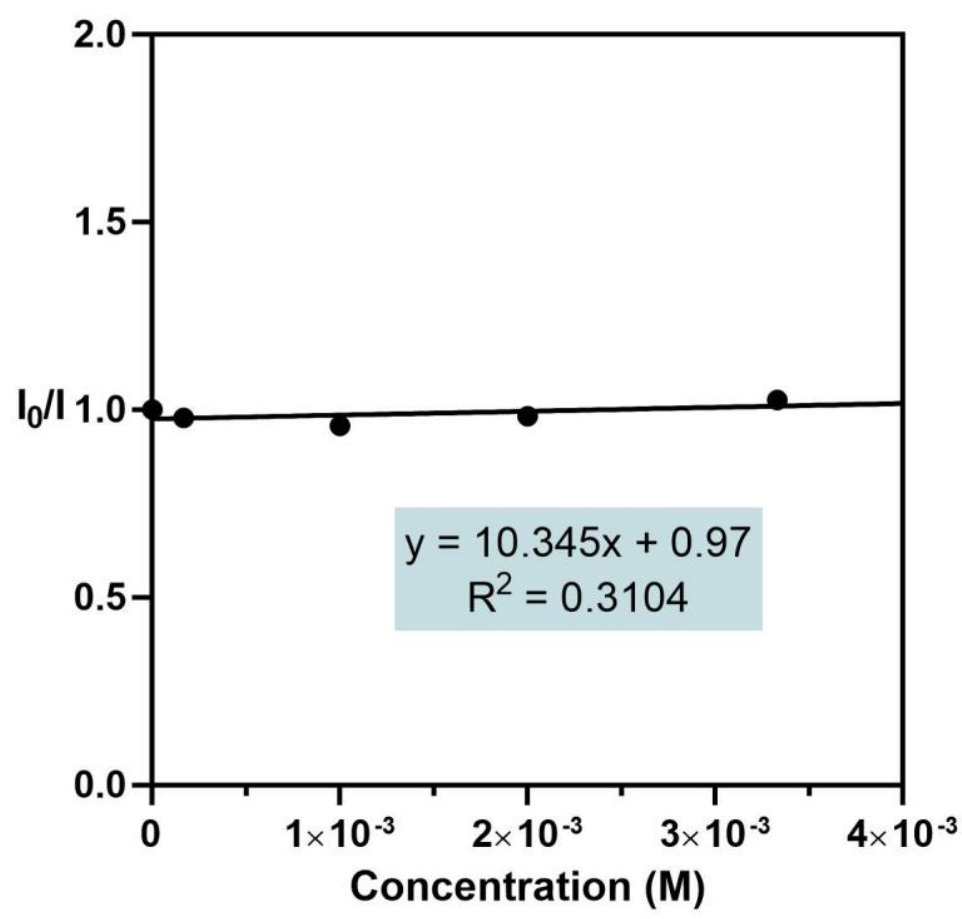

\begin{tabular}{cc}
\hline Acid and Base $\times 10^{4} \mathrm{M}$ & Intensity \\
\hline 0 & 699.59 \\
1.6 & 715.46 \\
10 & 730.43 \\
20 & 711.63 \\
33 & 681.63 \\
\hline
\end{tabular}

Figure S12. Characteristic plot of $\operatorname{Ir}[\mathrm{dF}(\mathrm{Me}) \mathrm{ppy}]_{2}(\mathrm{dtbbpy}) \mathrm{PF}_{6}\left(2.0 \times 10^{-4} \mathrm{M}\right)$ emission quenching by 3-(4-fluorophenyl)propanoic acid and 2,6-lutidine. Base and acid were used in a one to one stoichiometry. 


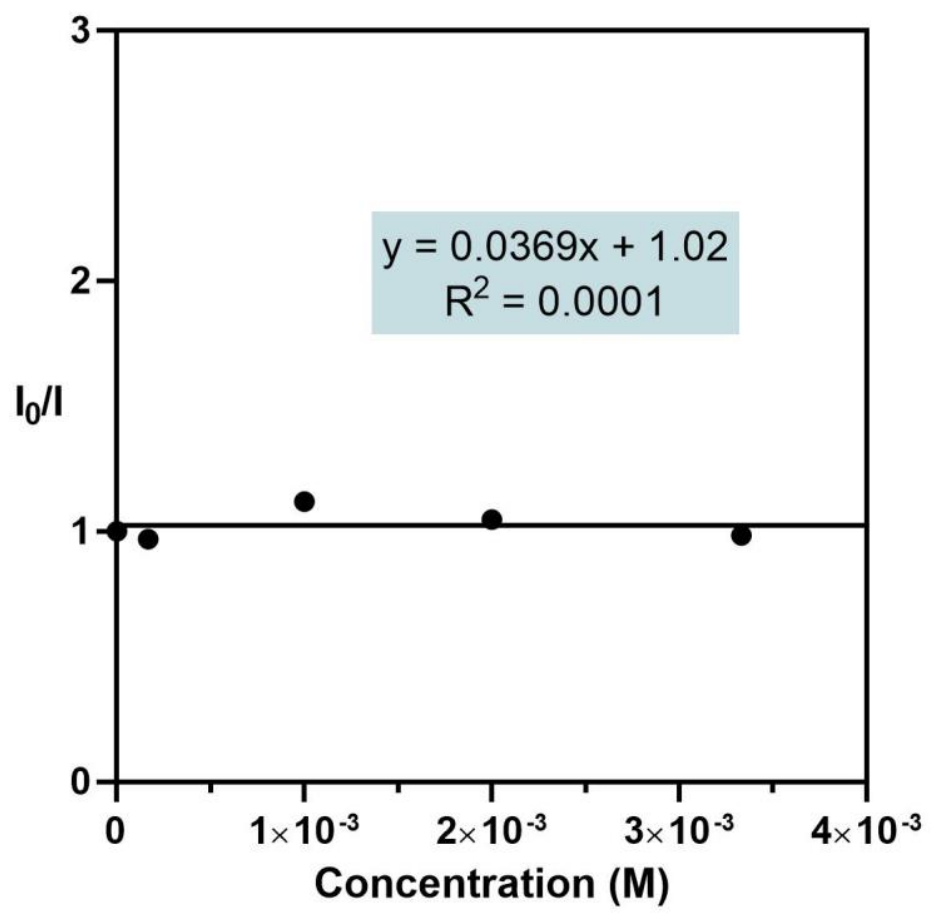

\begin{tabular}{cc}
\hline Diphenyl Disulfide $\times 10^{4} \mathbf{M}$ & Intensity \\
\hline 0 & 579.84 \\
1.6 & 598.76 \\
10 & 518.24 \\
20 & 553.33 \\
33 & 588.77 \\
\hline
\end{tabular}

Figure S13. Characteristic plot of $\operatorname{Ir}[\mathrm{dF}(\mathrm{Me}) \mathrm{ppy}]_{2}(\mathrm{dtbbpy}) \mathrm{PF}_{6}\left(2.0 \times 10^{-4} \mathrm{M}\right)$ emission quenching by diphenyl disulfide.

Our current rationale for the modest boost in yield with diphenyl disulfide is that it is capable of oxidizing $\operatorname{Ir}(\mathrm{II})$ to regenerate the ground-state $\operatorname{Ir}(\mathrm{III})$ photocatalyst. ${ }^{8}$ This likely increases the concentration of phosphine radical cation present in solution. It is possible that disulfide bond homolysis via blue-light irradiation could generate aryl thiyl radicals that are also capable of oxidizing $\operatorname{Ir}(\mathrm{II}) .^{9}$ 


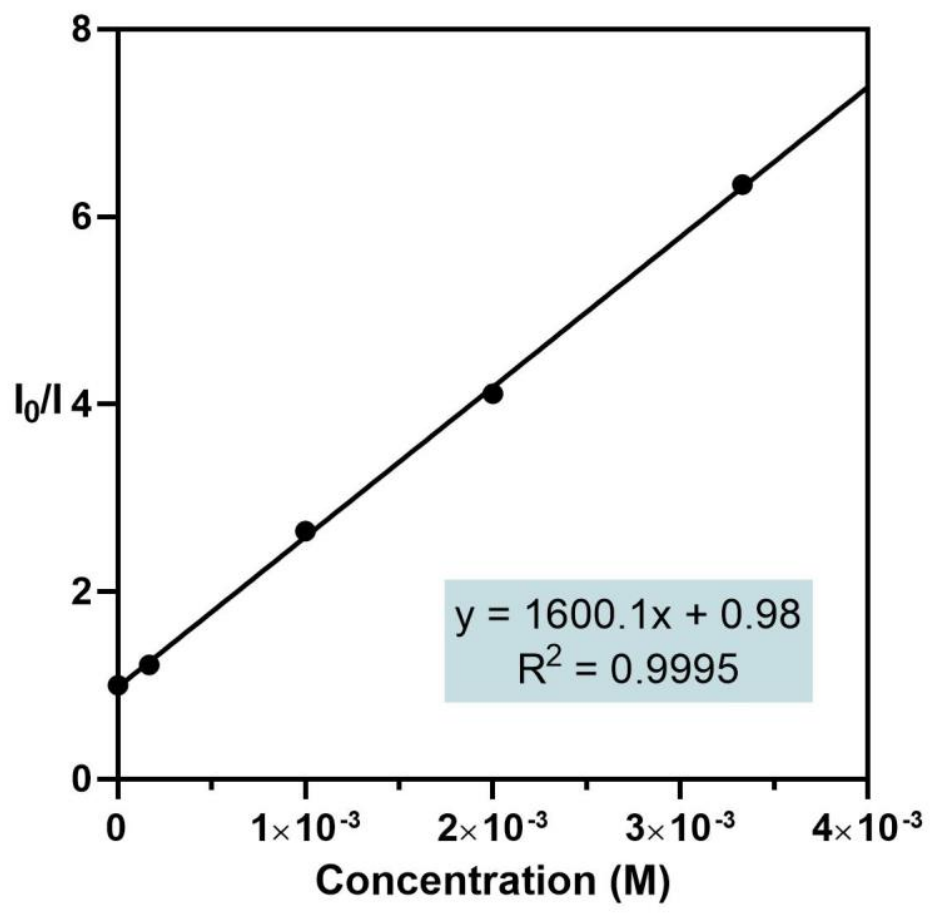

\begin{tabular}{ccc}
\hline Dimethyl Phenyl Phosphine $\times 10^{4} \mathrm{M}$ & Intensity & Intensity \\
\hline 0 & 567.34 & 578.92 \\
1.6 & 470.11 & 460.31 \\
10 & 227.22 & 203.00 \\
20 & 139.24 & 136.65 \\
33 & 87.64 & 91.27 \\
\hline
\end{tabular}

Figure S14. Characteristic plot of $\operatorname{Ir}[\mathrm{dF}(\mathrm{Me}) \mathrm{ppy}]_{2}(\mathrm{dtbbpy}) \mathrm{PF}_{6}\left(2.0 \times 10^{-4} \mathrm{M}\right)$ emission quenching by dimethyl phenyl phosphine. 


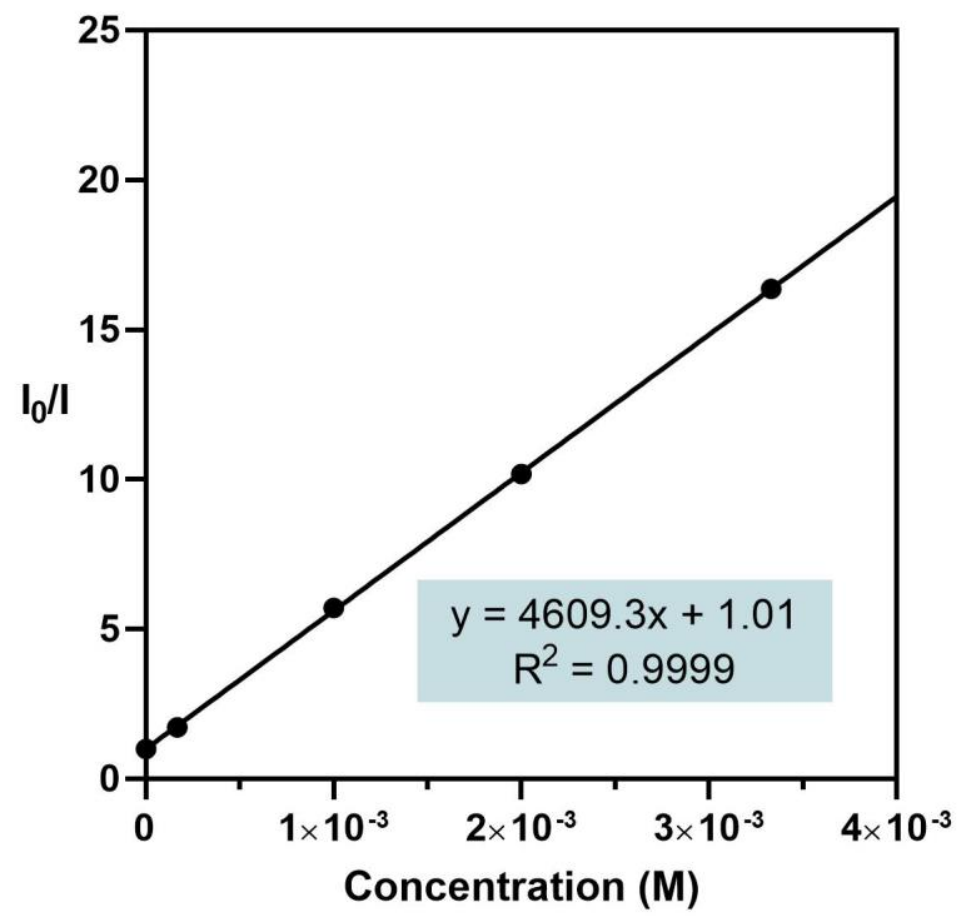

\begin{tabular}{ccc}
\hline Tris(4-methoxyphenyl) phosphine $\mathbf{x} 10^{4} \mathrm{M}$ & Intensity & Intensity \\
\hline 0 & 559.85 & 592.30 \\
1.6 & 341.76 & 326.78 \\
10 & 107.98 & 95.01 \\
20 & 60.60 & 53.03 \\
33 & 39.31 & 32.03 \\
\hline
\end{tabular}

Figure S15. Characteristic plot of $\operatorname{Ir}[\mathrm{dF}(\mathrm{Me}) \mathrm{ppy}]_{2}(\mathrm{dtbbpy}) \mathrm{PF} 6\left(2.0 \times 10^{-4} \mathrm{M}\right)$ emission quenching by tris(4-methoxyphenyl)phosphine. 


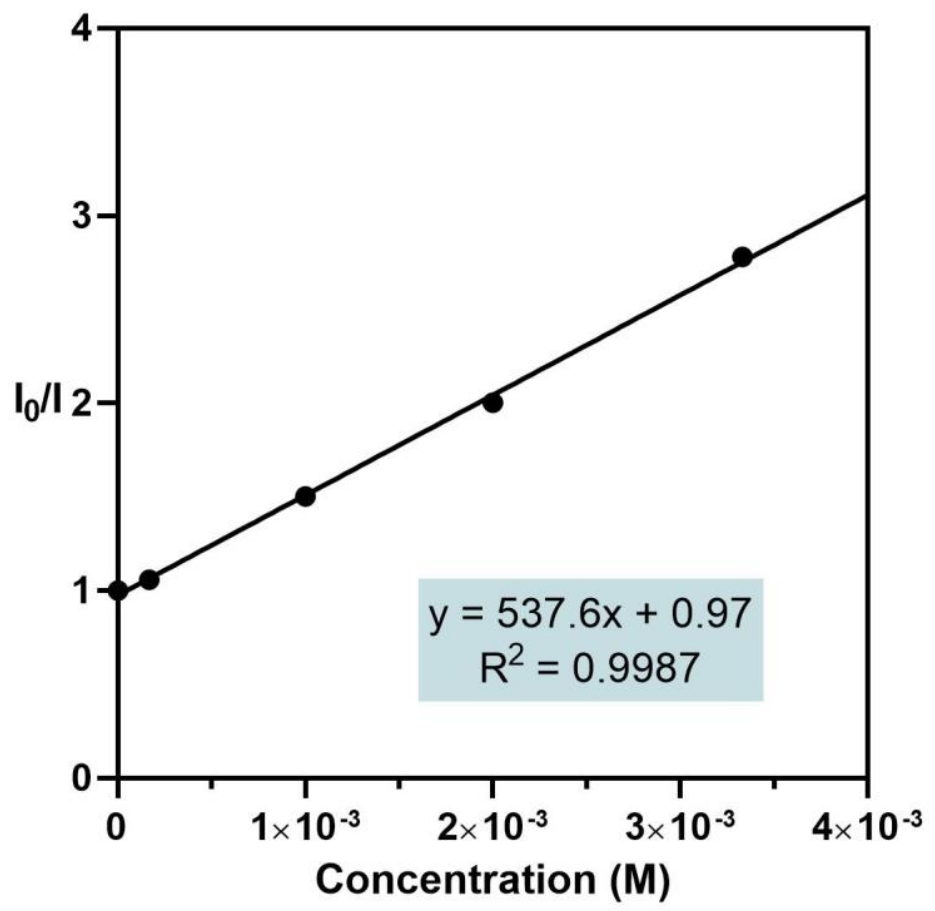

\begin{tabular}{ccc}
\hline Diphenyl Ethylene $\times 10^{4} \mathrm{M}$ & Intensity & Intensity \\
\hline 0 & 582.32 & 616.73 \\
1.6 & 564.06 & 567.05 \\
10 & 425.51 & 376.46 \\
20 & 312.27 & 288.12 \\
33 & 212.61 & 218.53 \\
\hline
\end{tabular}

Figure S16. Characteristic plot of $\operatorname{Ir}[\mathrm{dF}(\mathrm{Me}) \mathrm{ppy}]_{2}(\mathrm{dtbbpy}) \mathrm{PF}_{6}\left(2.0 \times 10^{-4} \mathrm{M}\right)$ emission quenching by diphenyl ethylene. 


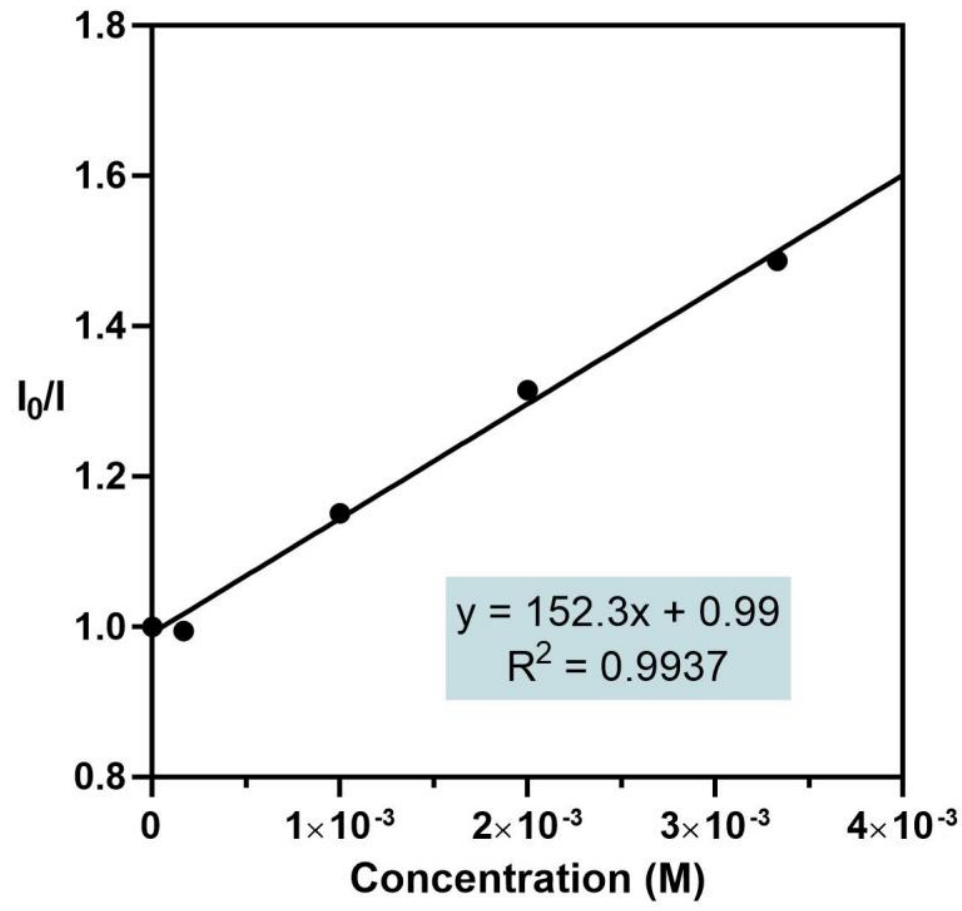

\begin{tabular}{ccc}
\hline Ethyl Diphenyl Phosphine $\times 10^{4} \mathrm{M}$ & Intensity & Intensity \\
\hline 0 & 600.61 & 596.31 \\
1.6 & 595.94 & 575.22 \\
10 & 501.65 & 455.90 \\
20 & 429.33 & 428.34 \\
33 & 358.68 & 428.34 \\
\hline
\end{tabular}

Figure S17. Characteristic plot of $\operatorname{Ir}[\mathrm{dF}(\mathrm{Me}) \mathrm{ppy}]_{2}(\mathrm{dtbbpy}) \mathrm{PF}_{6}\left(2.0 \times 10^{-4} \mathrm{M}\right)$ emission quenching by ethyl diphenyl phosphinite. 


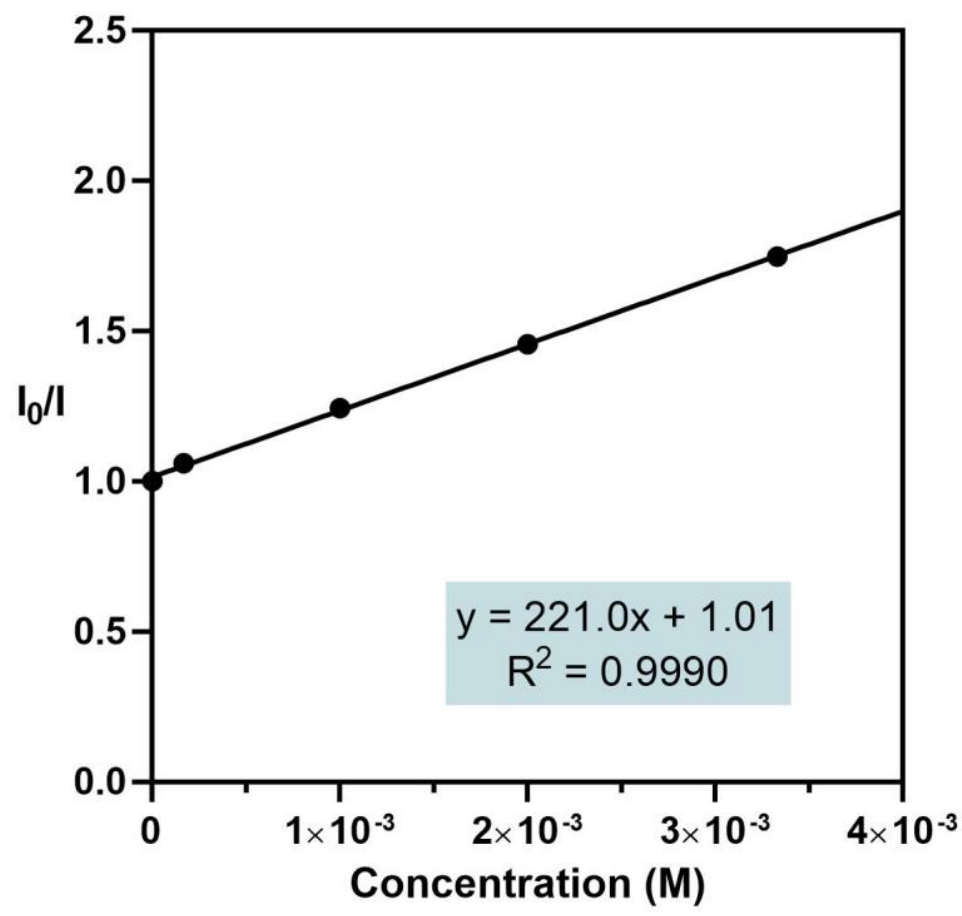

\begin{tabular}{ccc}
\hline Triphenyl Phosphine $\times 10^{4} \mathrm{M}$ & Intensity & Intensity \\
\hline 0 & 650.75 & 589.15 \\
1.6 & 606.73 & 562.54 \\
10 & 498.64 & 498.31 \\
20 & 422.51 & 429.43 \\
33 & 340.01 & 372.26 \\
\hline
\end{tabular}

Figure S18. Characteristic plot of $\operatorname{Ir}[\mathrm{dF}(\mathrm{Me}) \mathrm{ppy}]_{2}(\mathrm{dtbbpy}) \mathrm{PF}_{6}\left(2.0 \times 10^{-4} \mathrm{M}\right)$ emission quenching by triphenyl phosphine. 


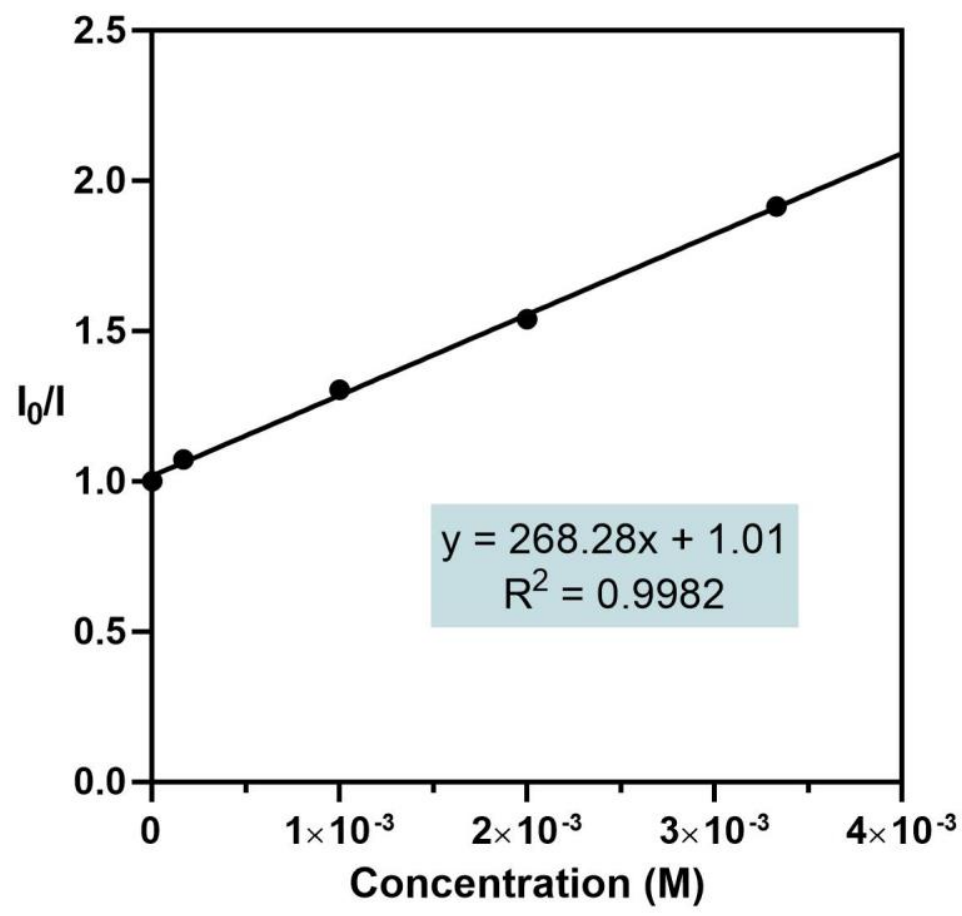

\begin{tabular}{cc}
\hline 1-(trifluoromethyl)-2-vinylbenzene $\times 10^{4} \mathrm{M}$ & Intensity \\
\hline 0 & 650.75 \\
1.6 & 606.73 \\
10 & 498.64 \\
20 & 422.51 \\
33 & 340.01 \\
\hline
\end{tabular}

Figure S19. Characteristic plot of $\operatorname{Ir}[\mathrm{dF}(\mathrm{Me}) \mathrm{ppy}]_{2}(\mathrm{dtbbpy}) \mathrm{PF}_{6}\left(2.0 \times 10^{-4} \mathrm{M}\right)$ emission quenching by 1 -(trifluoromethyl)-2-vinylbenzene. 


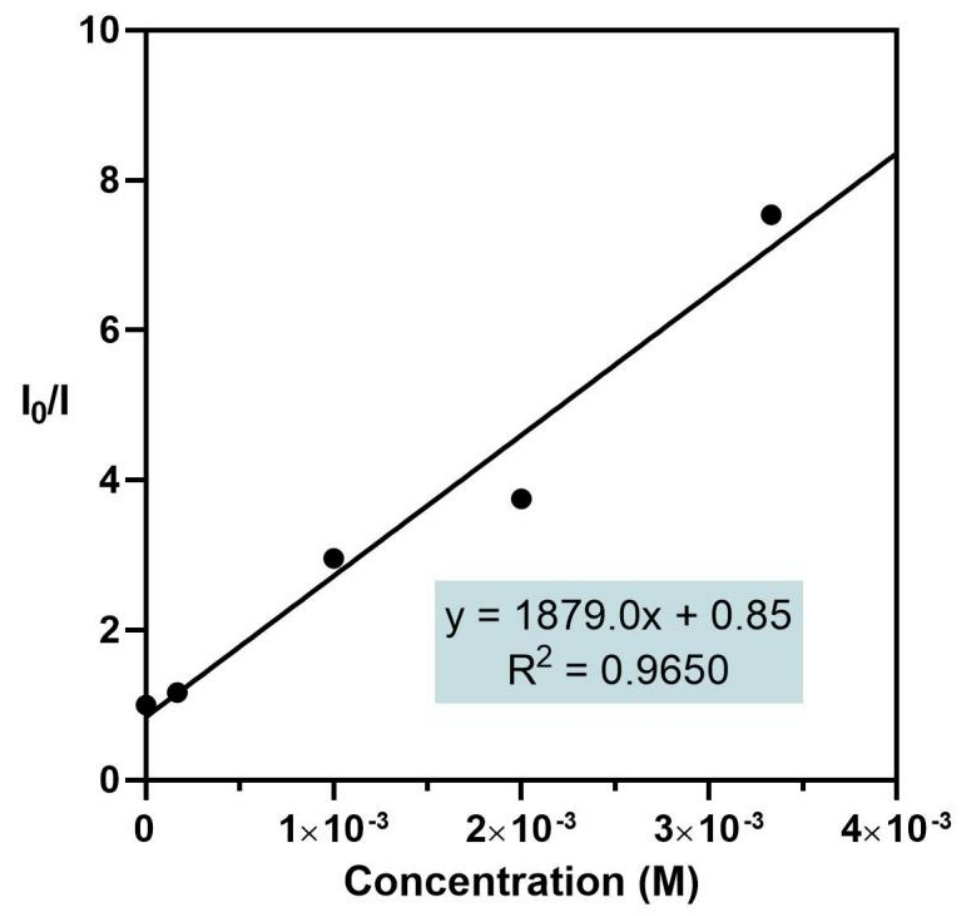

\begin{tabular}{cc}
\hline Reaction Mixture $\times 10^{4} \mathrm{M}$ & Intensity \\
\hline 0 & 587.01 \\
1.6 & 503.09 \\
10 & 198.46 \\
20 & 156.36 \\
33 & 77.86 \\
\hline
\end{tabular}

Figure S20. Characteristic plot of $\left.\operatorname{Ir}[\mathrm{dF}(\mathrm{Me}))_{\mathrm{ppy}}\right]_{2}(\mathrm{dtbbpy}) \mathrm{PF}_{6}\left(2.0 \times 10^{-4} \mathrm{M}\right)$ emission quenching by the reaction mixture. Aliquots of the reaction mixture were added relative to phosphine. The mixture contained dimethyl phenyl phosphine (2 equiv), 2,6-lutidine (1.0 equiv), 3-(4-fluorophenyl)propanoic acid (1.0 equiv), dipheny disulfide ( $5 \mathrm{~mol} \%)$, and diphenyl ethylene (3 equiv). 


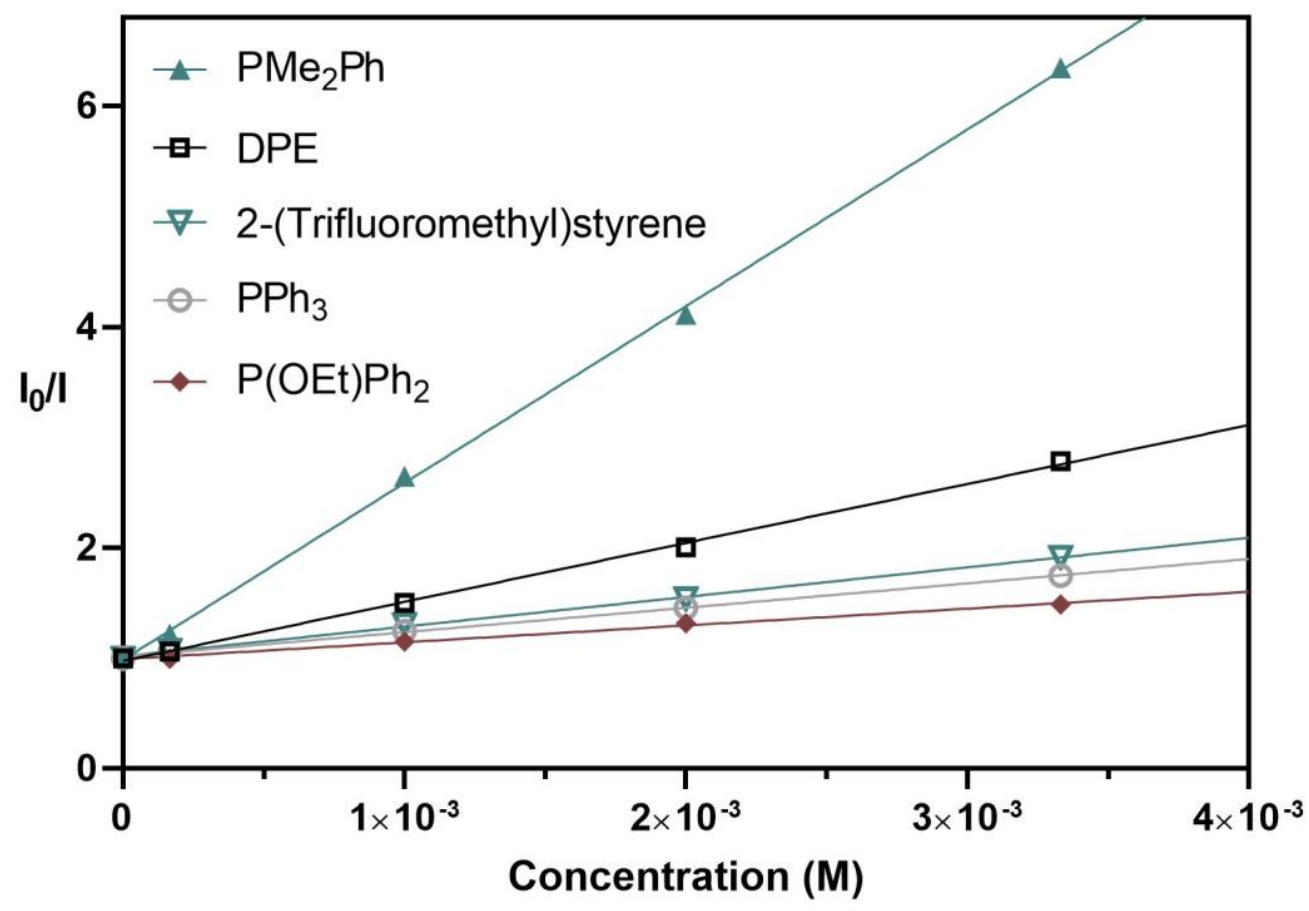

Figure S21. Overlay plot of $\operatorname{Ir}\left[\mathrm{dF}(\mathrm{Me}) \mathrm{ppy}_{2}\right]_{2}(\mathrm{dtbbpy}) \mathrm{PF}_{6}$ emission quenching by dimethyl phenyl phosphine, diphenyl ethylene, 1-(trifluoromethyl)-2-vinylbenzene, triphenyl phosphine, and ethyl diphenyl phosphinite.

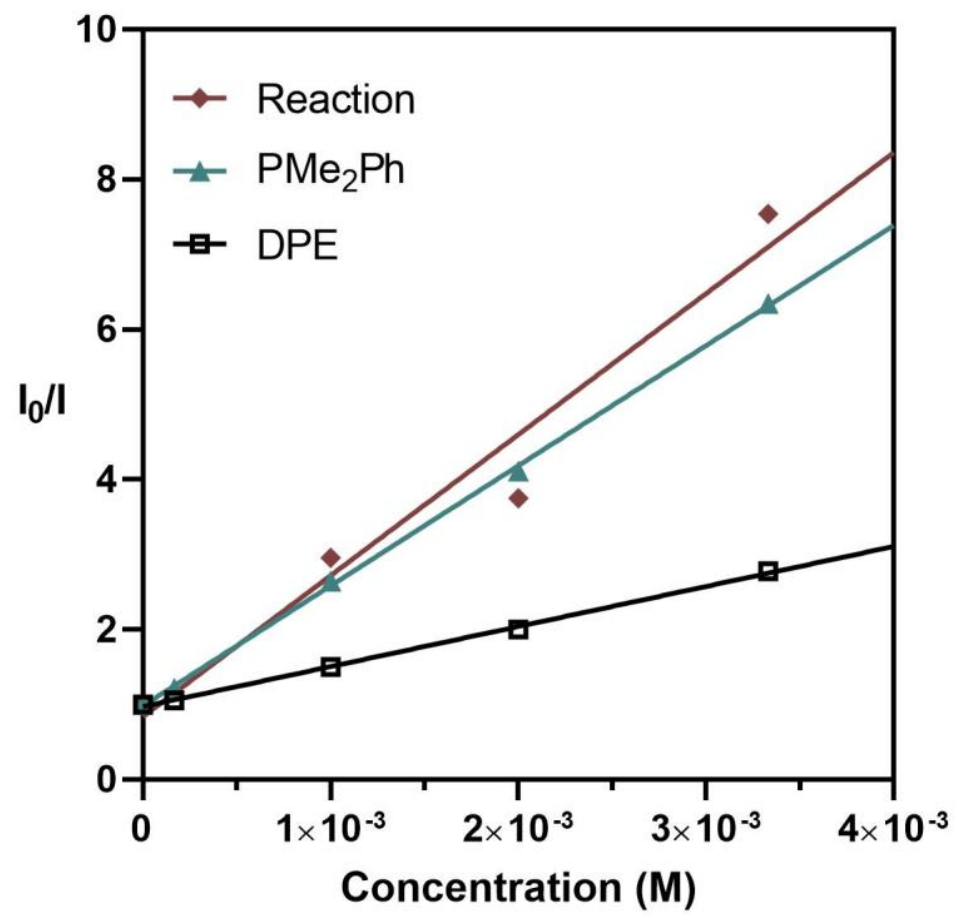

Figure S22. Overlay plot of $\operatorname{Ir}\left[\mathrm{dF}(\mathrm{Me}) \mathrm{ppp}_{2}\right]_{2}(\mathrm{dtbbpy}) \mathrm{PF}_{6}$ emission quenching by the reaction, dimethyl phenyl phosphine, and diphenyl ethylene.

Based on the Stern-Volmer quenching constants, it is likely that competitive electron transfer between dimethyl phenyl phosphine and an alkene takes place during the course of the reaction. Moreover, we propose that the higher quenching rate of dimethyl phenyl phosphine to quench 
the excited state of iridium relative to diphenyl ethylene is crucial for productive chemistry to occur. Likewise, the closer proximity of the Stern-Volmer quenching constant of ethyl diphenyl phosphinite to 1-(trifluoromethyl)-2-vinylbenzene than diphenyl ethylene leads to an increased efficiency in the coupling with 3-(4-fluorophenyl)propanoic acid with 1-(trifluoromethyl)-2-vinylbenzene (Table S10).

\section{B. Deuterium Studies}

General procedure for deuterium experiments. An oven-dried 1-dram reaction vial (VWR $\AA$ glass vials, 66011-041) was charged with carboxylic acid ( $0.5 \mathrm{mmol}, 1.0$ equiv) and equipped with a PTFE-coated stir bar (VWR® Micro stir bars, $2 \times 7 \mathrm{~mm}, 58948-976)$. The vial was Teflon taped on the threads, and then taken into a $\mathrm{N}_{2}$-filled glovebox. To the vial was added $\mathrm{MeCN}$ ( $0.5 \mathrm{M}), 1,1$-diphenyl ethylene ( $265 \mu \mathrm{L}, 1.5 \mathrm{mmol}, 3.0$ equiv) and pyridine $(40 \mu \mathrm{L}, 0.5 \mathrm{mmol}$, 1.0 equiv). Pyridine was used instead of 2,6-lutidine to prevent other hydrogen atom transfer reagents from interfering with the reaction. From a stock solution was added $\operatorname{Ir}\left[\mathrm{dF}(\mathrm{Me}) \mathrm{ppy}_{2}(\mathrm{dtbbpy}) \mathrm{PF}_{6}\right.$ ( $8.7 \mathrm{mg}, 0.01 \mathrm{mmol}, 0.017$ equiv). Finally, dimethyl phenyl phosphine ( $171 \mu \mathrm{L}, 1.2 \mathrm{mmol}, 2.4$ equiv) was added. The vial was then capped and sealed with electrical tape. The vial was irradiated for $24 \mathrm{~h}$ in a Photoreactor $(800 \mathrm{rpm}, 1500$ fan speed, $100 \%$ light intensity). An aliquot of the crude reaction mixture was analzed by ${ }^{1} \mathrm{H}-\mathrm{NMR}$ with 1 fluoronaphthalene ( $65 \mu \mathrm{L}, 0.5 \mathrm{mmol}, 1.0$ equiv) as an external standard. Quantitative carbon was used for calculating deuterium incorporation.

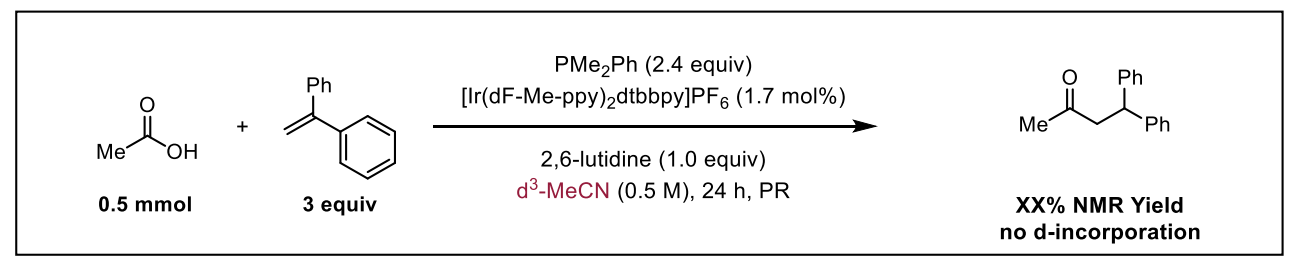




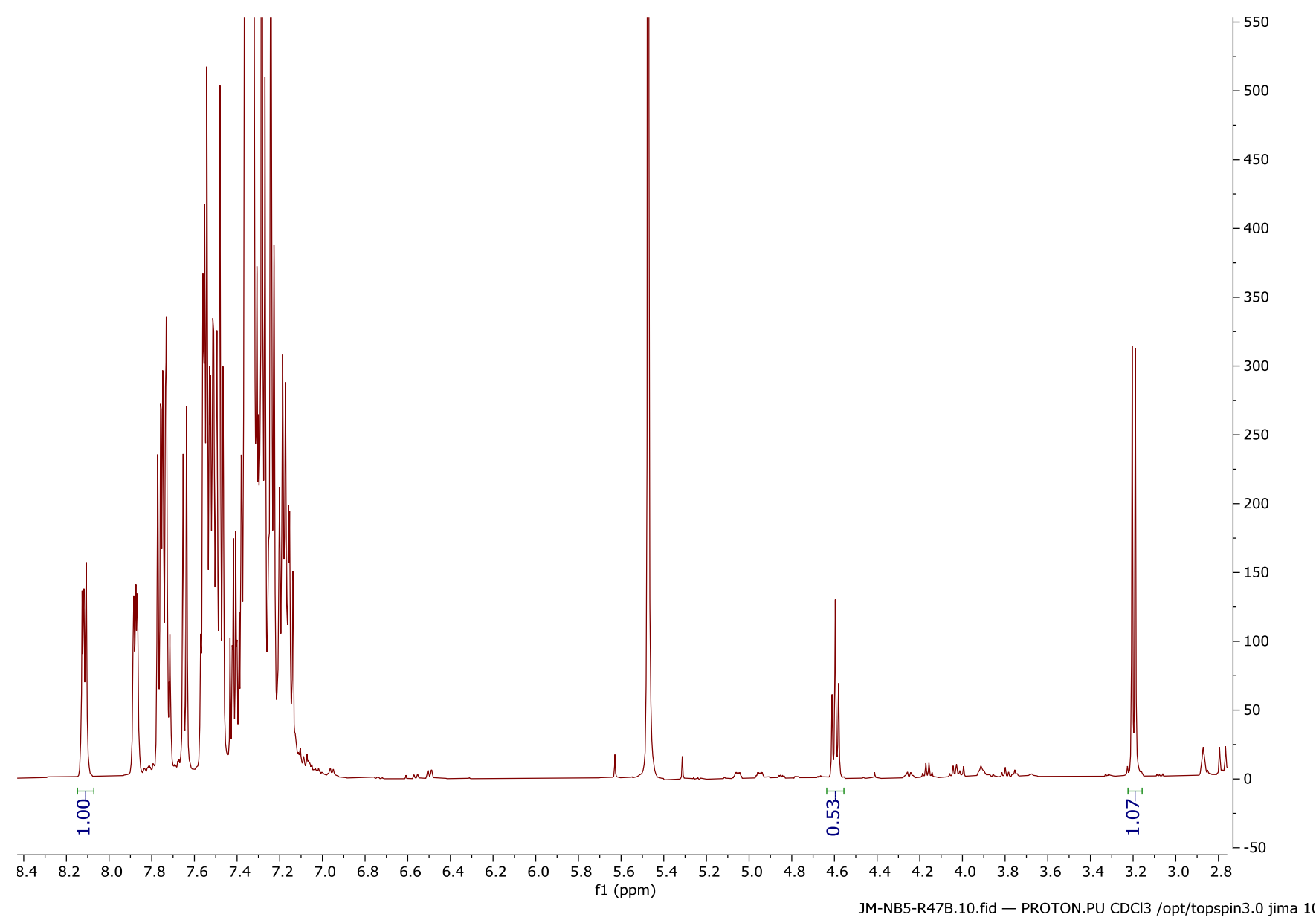

Figure S23. Crude reaction mixture of acetic acid and diphenyl ethylene in $\mathrm{d}_{3}-\mathrm{MeCN}$ after 24 hours.

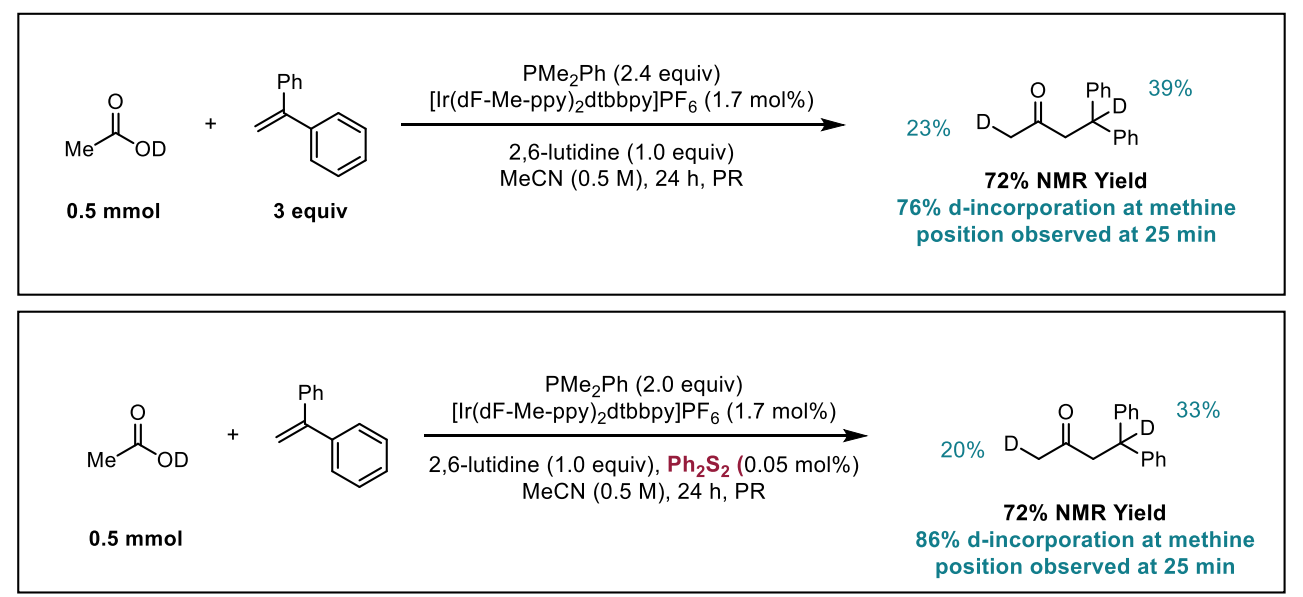

Figure S24. A. The reaction of monodeuteroacetic acid with diphenyl ethylene in MeCN. B. The reaction of monodeuteroacetic acid with diphenyl ethylene in $\mathrm{MeCN}$ with diphenyl disulfide. 


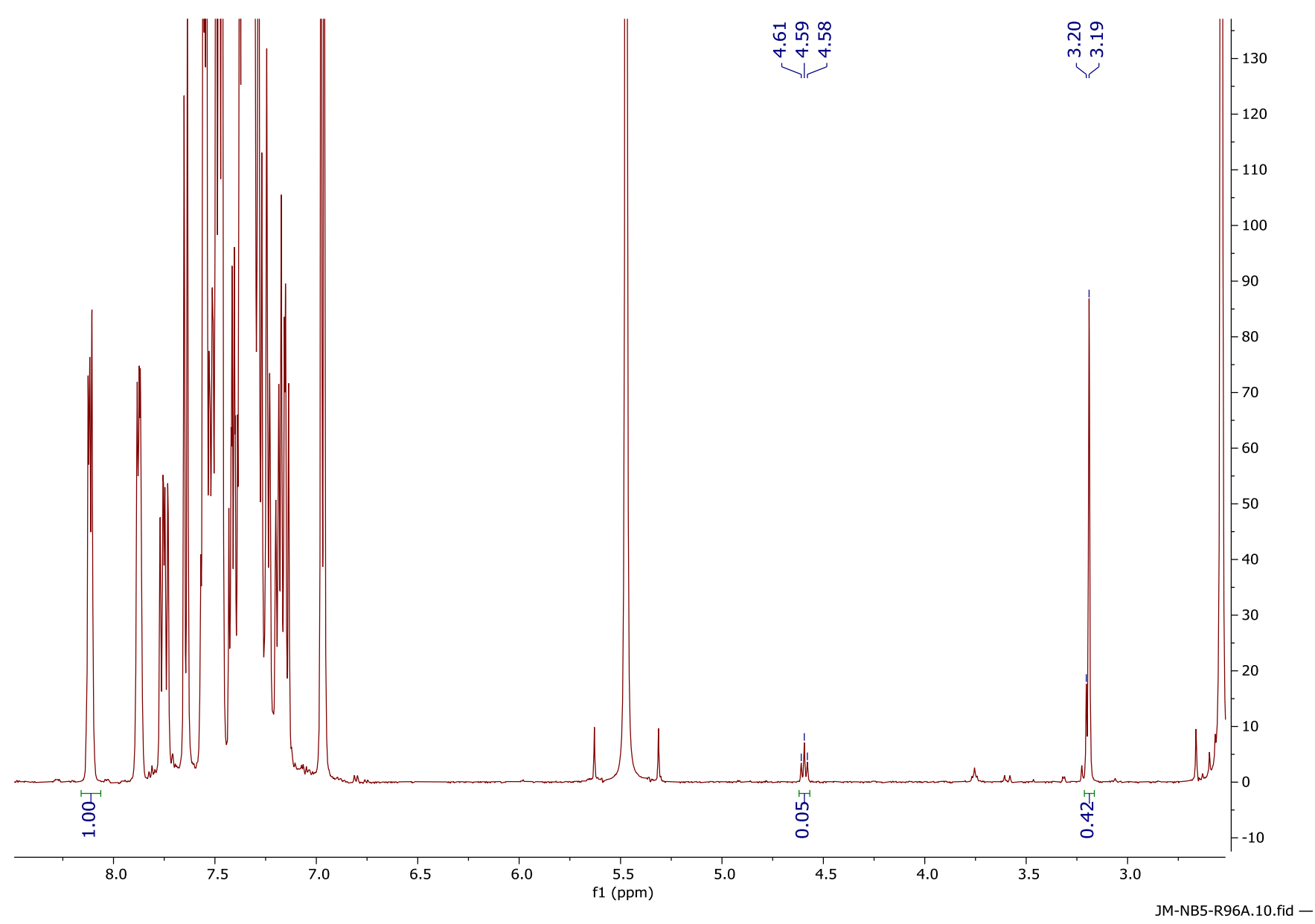

Figure S25. Crude reaction mixture of monodeuteroacetic acid and diphenyl ethylene after 25 minutes when diphenyl disulfide is excluded. The crude ${ }^{1} \mathrm{H}$-NMR using 1 -fluoronaphthalene as standard reveals $76 \%$ deuterium incorporation. 


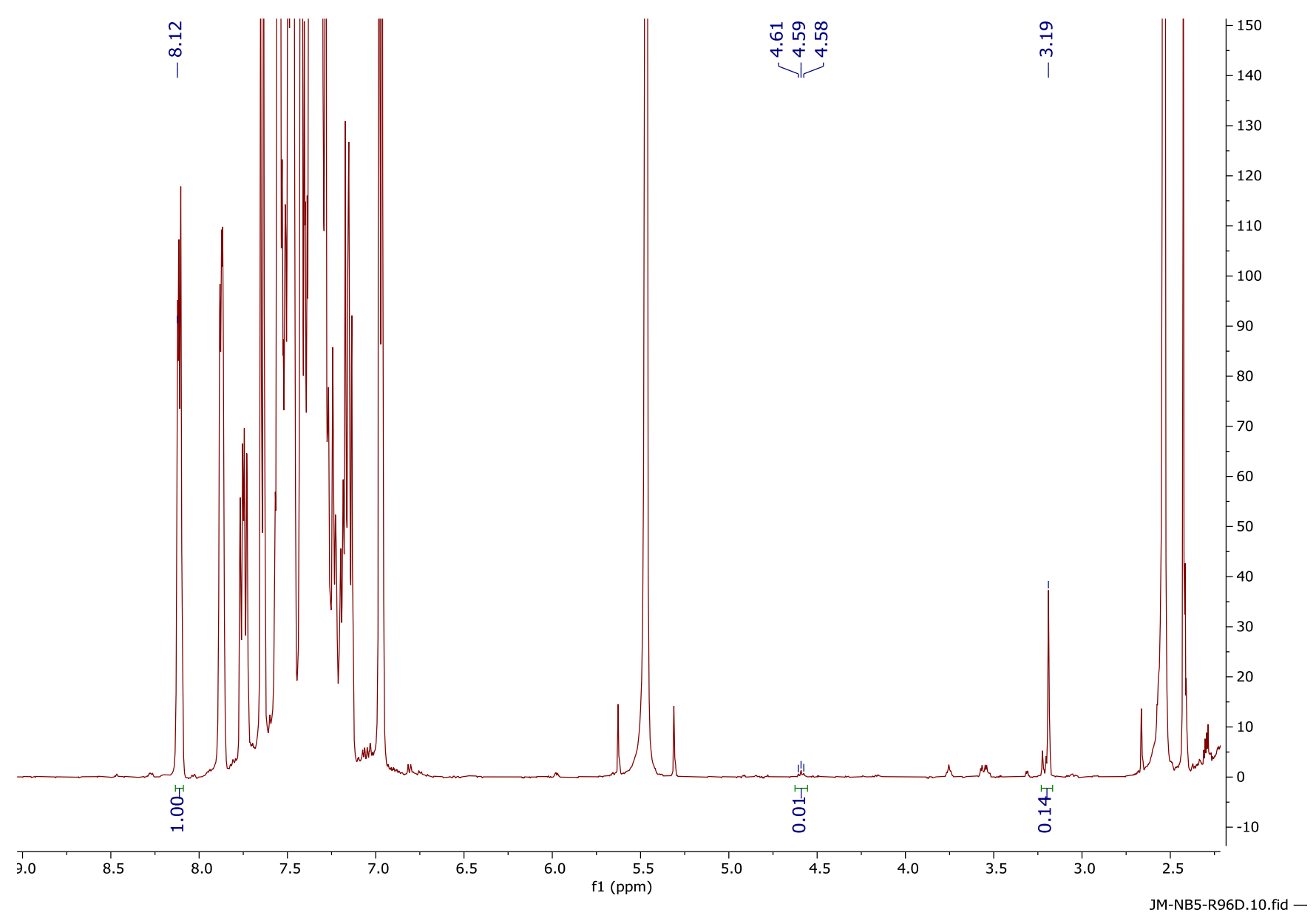

Figure S26. Crude reaction mixture of monodeuteroacetic acid and diphenyl ethylene after 25 minutes when diphenyl disulfide is included. The crude ${ }^{1} \mathrm{H}$-NMR using 1 -fluoronaphthalene as standard reveals $86 \%$ deuterium incorporation. 


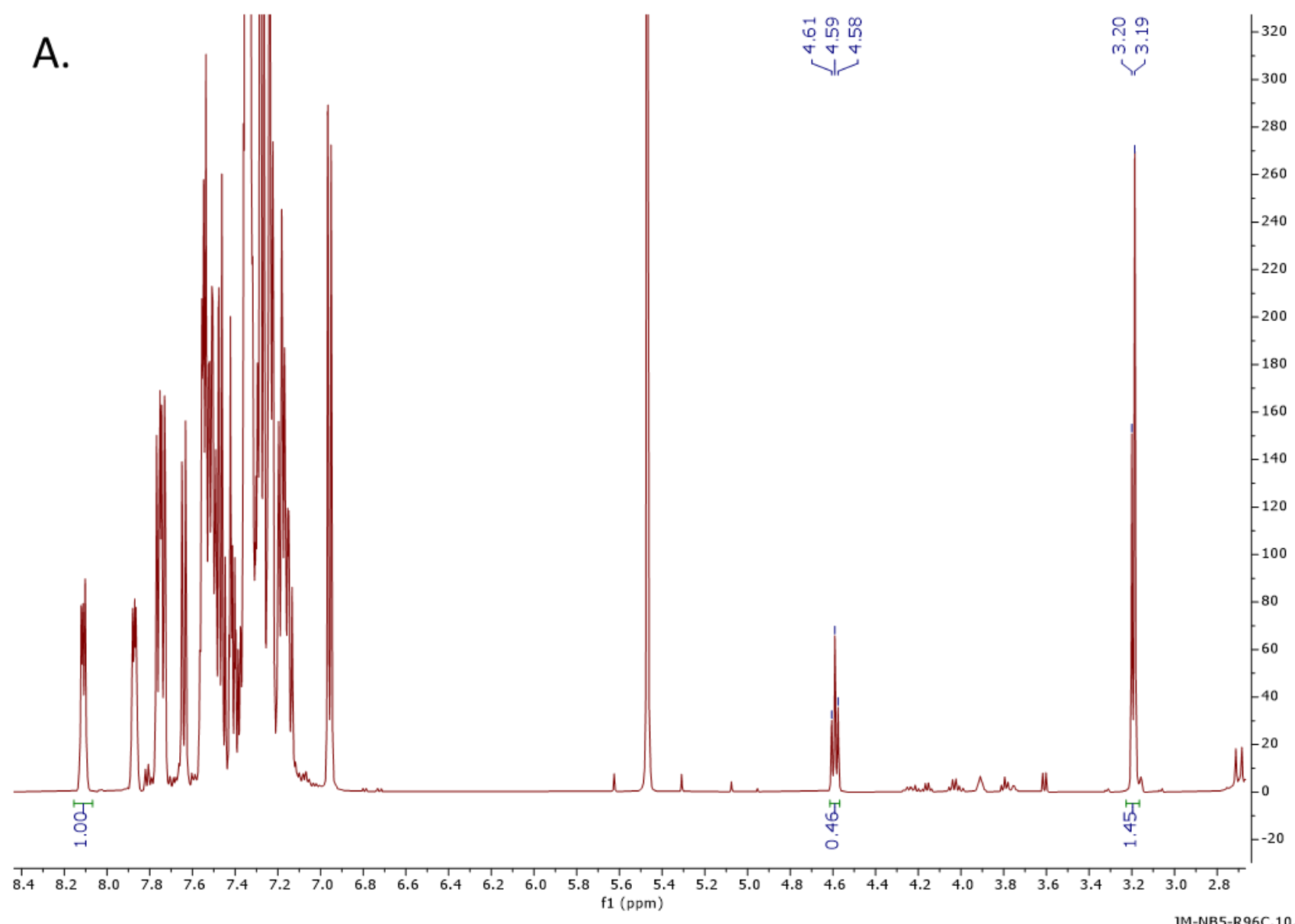

B.

Name: M1

From: $49.210 \mathrm{ppm}$

To: $49.897 \mathrm{ppm}$

Residual Error:59.3

\# ppm Height Width(Hz) L/G Area

$\begin{array}{lll}1 & 49.6521446 .741 .7 & 0.194886 .005\end{array}$

$249.5626212 .882 .1 \quad 0.542758 .065$

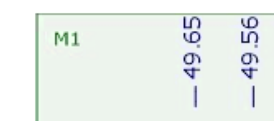

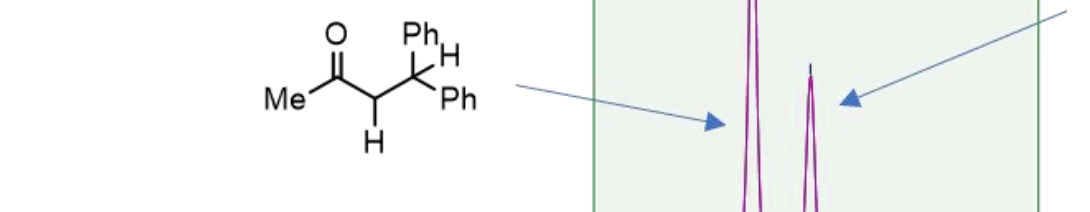

$\longrightarrow$

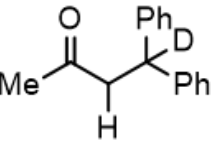

JM-NB5-R96C.10.fid -

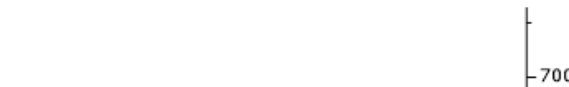

$-700$

$-600$

500

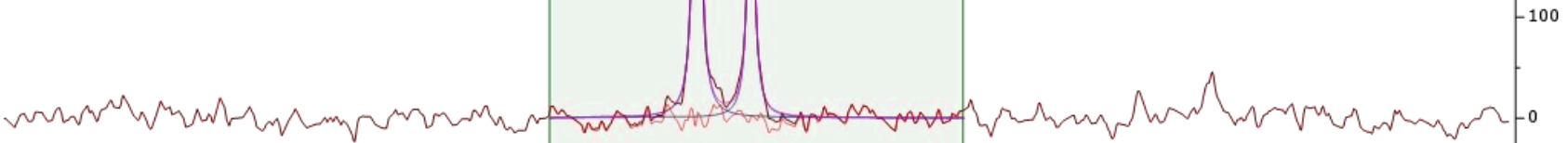

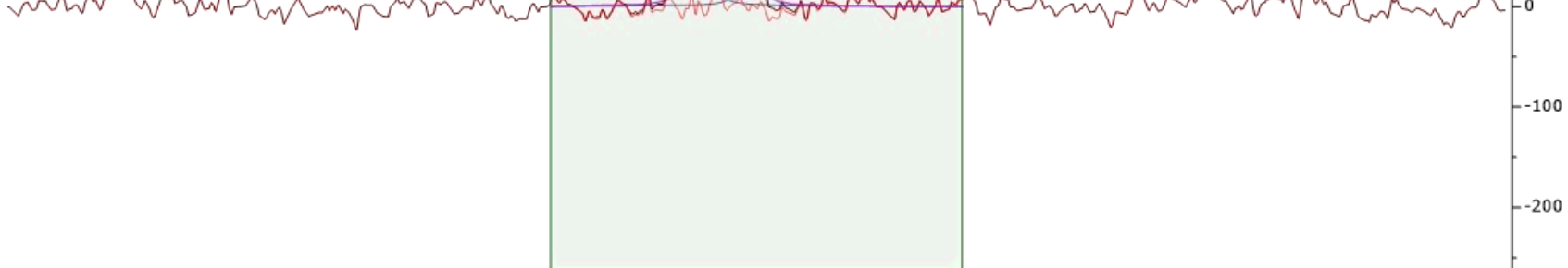

\begin{tabular}{lllllllllllllllllllllllllllllllllll}
\hline$j .8$ & 50.7 & 50.6 & 50.5 & 50.4 & 50.3 & 50.2 & 50.1 & 50.0 & 49.9 & 49.8 & 49.7 & 49.6 & 49.5 & 49.4 & 49.3 & 49.2 & 49.1 & 49.0 & 48.9 & 48.8 & 48.7 & 48.6 & 48.5 & 48.4
\end{tabular} f1 (ppm)

JM-NB5-R96C.13.fid - 


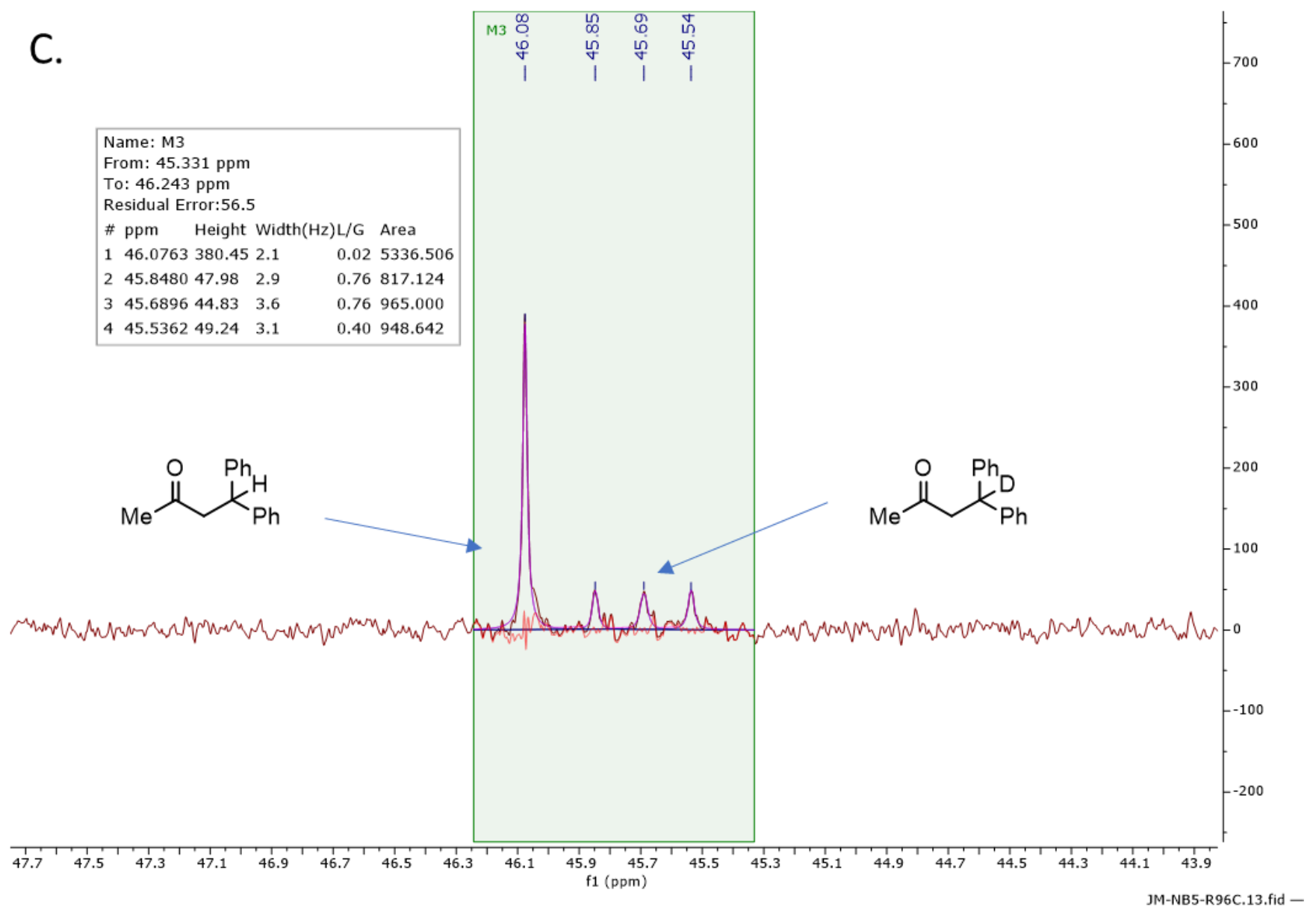


D.

Name: M2

From: $30.164 \mathrm{ppm}$

To: $30.852 \mathrm{ppm}$

Residual Error:86.4

\# ppm Height Width(Hz) L/G Area

130.6896616 .802 .0

$\begin{array}{lll}2 & 30.590754 .59 & 2.2\end{array}$

$\begin{array}{lll}3 & 30.438057 .60 \quad 2.6\end{array}$

$\begin{array}{lll}4 & 30.281248 .86 & 2.6\end{array}$

0.617298 .625

2.00586 .015

2.00716 .632

$5 \quad 30.2467108 .171 .7$

2.00609 .261

0.731075 .536<smiles>[2H]CC(=O)CC(c1ccccc1)c1ccccc1</smiles>

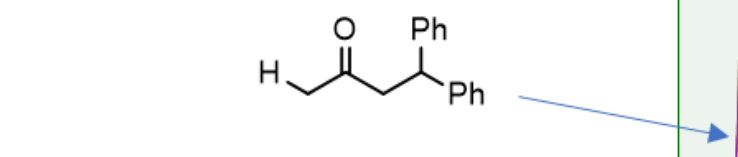

Figure S27. Crude reaction mixture of monodeuteroacetic acid and diphenyl ethylene after 24 hours. Phenyl disulfide was included in this reaction. A. The crude ${ }^{1} \mathrm{H}-\mathrm{NMR}$ using 1 -fluoronaphthalene as standard. B. The quantitative ${ }^{13} \mathrm{C}-\mathrm{NMR}$ of the secondary alpha $\mathrm{C}-\mathrm{H}$ bond. C. The quantitative ${ }^{13} \mathrm{C}$-NMR centered about the methine carbon of the product. $\mathrm{D}$. The quantitative ${ }^{13} \mathrm{C}-\mathrm{NMR}$ centered about the primary alpha carbon of the carbonyl. All quantitative carbon signals show an isotropic shift associated with deuteration.

In order to further probe the configurational stability of enolizable $\mathrm{C}-\mathrm{H}$ bonds in the product, an enantioenriched derivative of nipecotic acid was subjected to the reaction conditions (Table S11). An erosion of e.e. was measured in the product during the reaction conditions. Notably, the enantioenriched ketone maintains the same level of e.e. when cyclohexane carboxylic acid is coupled with diphenyl ethylene (Figure S28).

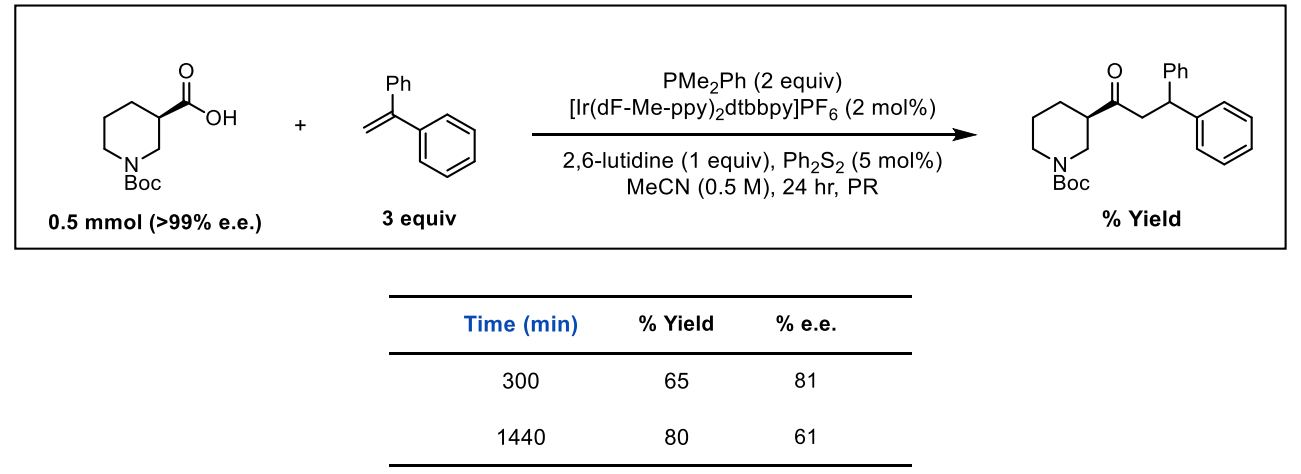

Table S11. Reaction of an enantioenriched derivative of nipecotic acid with diphenyl ethylene. 


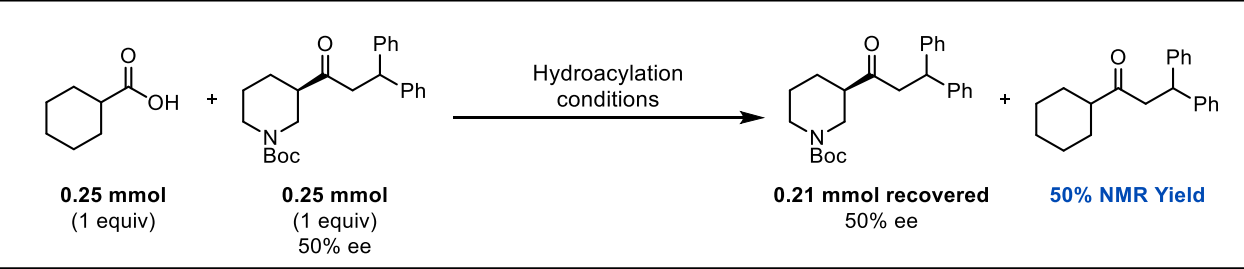

Figure S28. Reaction between cyclohexane carboxylic acid and diphenyl ethylene with the product of nipecotic acid added as an additive.
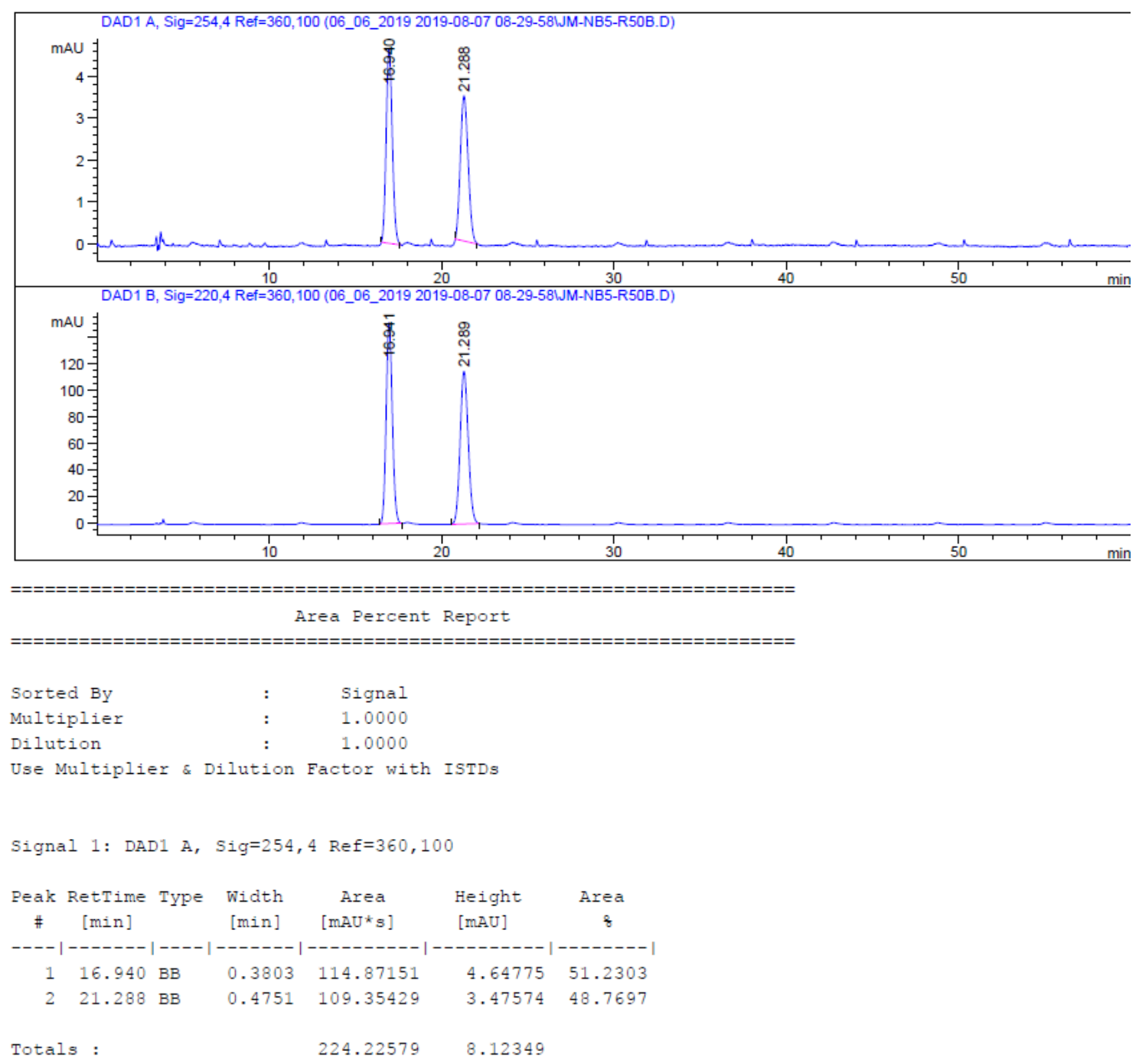
Figure S29. Racemic standard of product. ChiralPak ${ }^{\circledR}$ IC, $5 \%$ IPA in Hexanes, 60 min run, 1 $\mathrm{mL} / \mathrm{min}$.

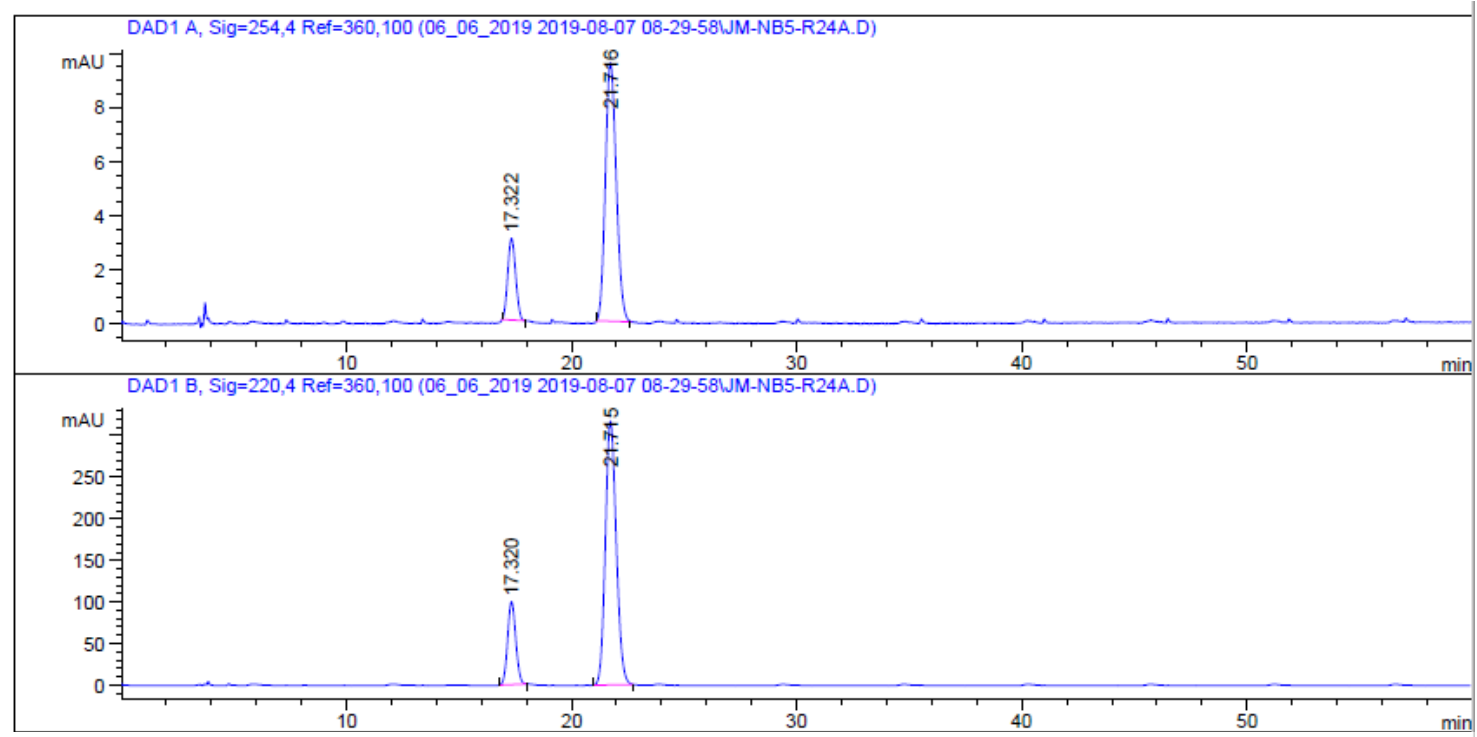

Area Percent Report

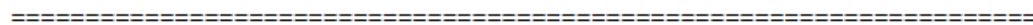

$\begin{array}{lll}\text { Sorted By } & : & \text { Signal } \\ \text { Multiplier } & : & 1.0000 \\ \text { Dilution } & : & 1.0000\end{array}$

Use Multiplier \& Dilution Factor with ISTDs

Signal 1: DAD1 A, Sig=254, 4 Ref $=360,100$

\begin{tabular}{|c|c|c|c|c|c|c|}
\hline $\begin{array}{c}\text { Peak } \\
\quad \#\end{array}$ & $\begin{array}{c}\text { RetTime } \\
\text { [min] }\end{array}$ & Type & $\begin{array}{l}\text { Width } \\
\text { [min] }\end{array}$ & $\begin{array}{c}\text { Area } \\
{\left[\mathrm{mAU}^{*} \mathrm{~s}\right]}\end{array}$ & $\begin{array}{l}\text { Height } \\
\text { [mAU] }\end{array}$ & $\begin{array}{c}\text { Area } \\
\&\end{array}$ \\
\hline \multicolumn{7}{|c|}{ 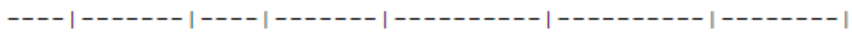 } \\
\hline 1 & 17.322 & $\mathrm{BB}$ & 0.3762 & 74.38911 & 3.03192 & 18.7797 \\
\hline 2 & 21.716 & $\mathrm{BB}$ & 0.5247 & 321.72604 & 9.55714 & 81.2203 \\
\hline 10 cat & Ls : & & & 396.11516 & 12.58906 & \\
\hline
\end{tabular}

Signal 2: DAD1 B, Sig=220, 4 Ref $=360,100$

\begin{tabular}{|c|c|c|c|c|c|c|}
\hline $\begin{array}{l}\text { Eak } \\
\#\end{array}$ & $\begin{array}{c}\text { RetTime } \\
\text { [min] }\end{array}$ & Type & $\begin{array}{l}\text { Width } \\
\text { [min] }\end{array}$ & $\begin{array}{c}\text { Area } \\
{\left[\mathrm{mAU}{ }^{*} \mathrm{~s}\right]}\end{array}$ & $\begin{array}{l}\text { Height } \\
\text { [mAU] }\end{array}$ & $\begin{array}{c}\text { Area } \\
\&\end{array}$ \\
\hline & 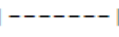 & & & $---------\mid$ & -- & . \\
\hline 1 & & & & 2521.13037 & 121 & 191 \\
\hline 2 & 21 & & & $1.06653 \mathrm{e} 4$ & 317.04135 & 8809 \\
\hline
\end{tabular}

Instrument $18 / 8 / 2019$ 2:58:18 PM MAB

Figure S30. Product of enantioenriched acid after $24 \mathrm{~h}$. ChiralPak ${ }^{\circledR} \mathrm{IC}, 5 \%$ IPA in Hexanes, 60 $\min$ run, $1 \mathrm{~mL} / \mathrm{min}$. 
Given the lack of deuterium incorporation when $\mathrm{d}_{3}-\mathrm{MeCN}$ was used as solvent, and the observed reactivity at enolizable $\mathrm{C}-\mathrm{H}$ bonds, a reduction/protonation sequence is proposed to close the catalytic cycle of this reaction.

\section{C. $\mathrm{CV}$ of $\mathrm{PMe}_{2} \mathrm{Ph}$}

Cyclic voltammetry was conducted on a $\mathrm{CH}$ Instruments Electrochemical Analyzer ( $\mathrm{CH} 1600 \mathrm{E})$. In a nitrogen filled glove box, a $1.3 \mathrm{mM}$ solution of $\mathrm{PMe}_{2} \mathrm{Ph}$ with $0.2 \mathrm{M}$ tetrabutylammonium hexafluorophosphate as supporting electrolyte in MeCN was prepared. The solution was removed from the glove box and a cyclic voltammogram was collected under a nitrogen atmosphere using a glassy carbon working electrode, a platinum mesh counder electrode, and a saturated calomel reference electrode.

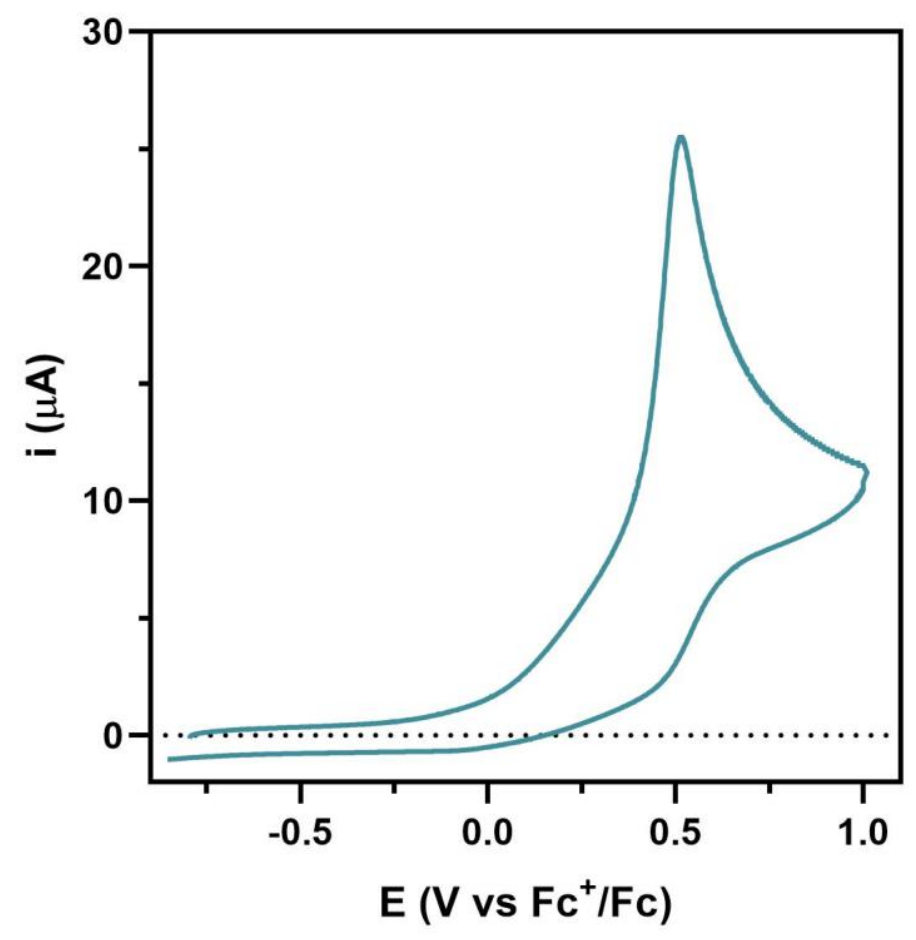

Figure S31. Cyclic Voltammogram of $\mathrm{PMe}_{2} \mathrm{Ph}\left(\mathrm{Scan}\right.$ rate $\left.0.1 \mathrm{Vs}^{-1}\right)$ shows an irreversible oxidation at $\mathrm{Ep}=0.51 \mathrm{~V}$ vs $\mathrm{Fc} / \mathrm{Fc}^{+}$, when ferrocene was used as an external reference $(\mathrm{Ep}=0.89 \mathrm{~V}$ vs SCE). ${ }^{10}$ 


\section{References}

(1) Le, C.; Wismer, M. K.; Shi, Z.-C.; Zhang, R.; Conway, D. V.; Li, G.; Vachal, P.; Davies, I. W.; MacMillan, D. W. A General Small-Scale Reactor To Enable Standardization and Acceleration of Photocatalytic Reactions. Acs Central Sci 2017, 3 (6), 647-653. https://doi.org/10.1021/acscentsci.7b00159 .

(2) Onuigbo, L.; Raviola, C.; Fonzo, A.; Protti, S.; Fagnoni, M. Sunlight-Driven Synthesis of Triarylethylenes (TAEs) via Metal-Free Mizoroki-Heck-Type Coupling. Eur J Org Chem 2018, 2018 (38), 5297-5303. https://doi.org/10.1002/ejoc.201800883 .

(3) Wang, G.-Z.; Li, X.-L.; Dai, J.-J.; Xu, H.-J. AlBN-Catalyzed Oxidative Cleavage of Gem Disubstituted Alkenes with $\mathrm{O} 2$ as an Oxidant. J Org Chem 2014, 79 (15), 7220-7225. https://doi.org/10.1021/jo501203a .

(4) Ainge, D.; Ennis, D.; Gidlund, M.; Stefinovic, M.; Vaz, L.-M. Rapid Development of an Enantioselective Synthesis of ( R )-1-Hydroxy-7-Methoxy-1,2,3,4-Tetrahydronaphthalene-1-Carboxylic Acid. Org Process Res Dev 2003, 7 (2), 198-201. https://doi.org/10.1021/op025590v .

(5) Pratsch, G.; Overman, L. E. Synthesis of 2,5-Diaryl-1,5-Dienes from Allylic Bromides Using Visible-Light Photoredox Catalysis. J Org Chem 2015, 80 (22), 11388-11397. https://doi.org/10.1021/acs.joc.5b01962 .

(6) Huhtiniemi, T.; Salo, H. S.; Suuronen, T.; Poso, A.; Salminen, A.; Leppänen, J.; Jarho, E.; Lahtela-Kakkonen, M. Structure-Based Design of Pseudopeptidic Inhibitors for SIRT1 and SIRT2. J Med Chem 2011, 54 (19), 6456-6468. https://doi.org/10.1021/jm200590k .

(7) Shields, B. J.; Doyle, A. G. Direct C(Sp3)-H Cross Coupling Enabled by Catalytic Generation of Chlorine Radicals. J Am Chem Soc 2016, 138 (39), 12719-12722. https://doi.org/10.1021/jacs.6b08397 .

(8) Samide, M. J.; Peters, D. G. Catalytic Reduction of Diphenyl Disulfide by Cobalt(I) Salen Electrogenerated at a Carbon Cathode in Acetonitrile. J Electrochem Soc 1998, 145 (10), 3374-3378. https://doi.org/10.1149/1.1838814 .

(9) Zhu, Q.; Graff, D. E.; Knowles, R. R. Intermolecular Anti-Markovnikov Hydroamination of Unactivated Alkenes with Sulfonamides Enabled by Proton-Coupled Electron Transfer. J Am Chem Soc 2018, 140 (2), 741-747. https://doi.org/10.1021/jacs.7b11144 .

(10) Pavlishchuk, V. V.; Addison, A. W. Conversion Constants for Redox Potentials Measured versus Different Reference Electrodes in Acetonitrile Solutions at $25^{\circ} \mathrm{C}$. Inorg Chim Acta 2000, 298 (1), 97-102. https://doi.org/10.1016/s0020-1693(99)00407-7 . 


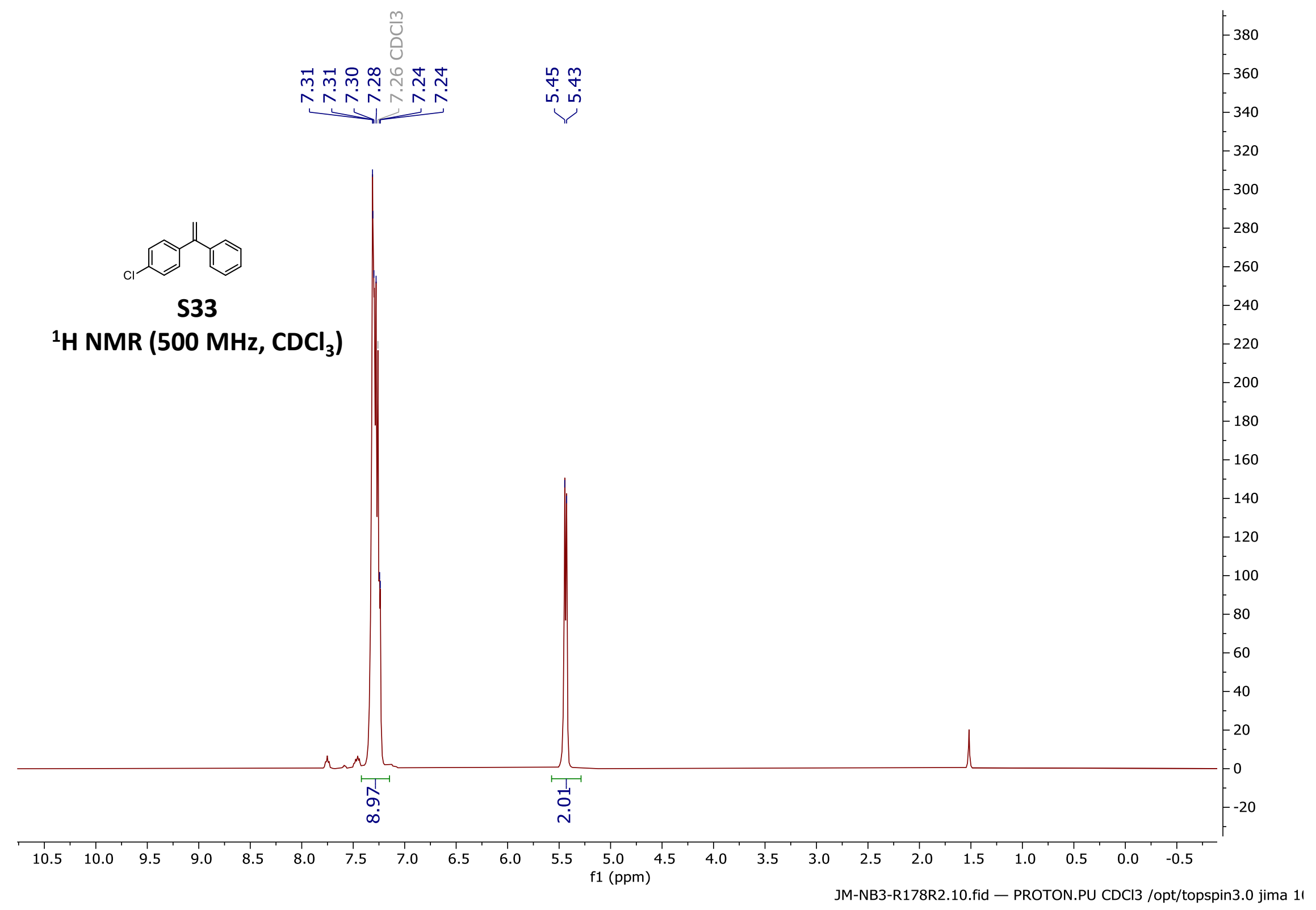


$\begin{array}{ll}m & -2200\end{array}$

S33

${ }^{13} \mathrm{C}$ NMR (126 MHz, $\left.\mathrm{CDCl}_{3}\right)$

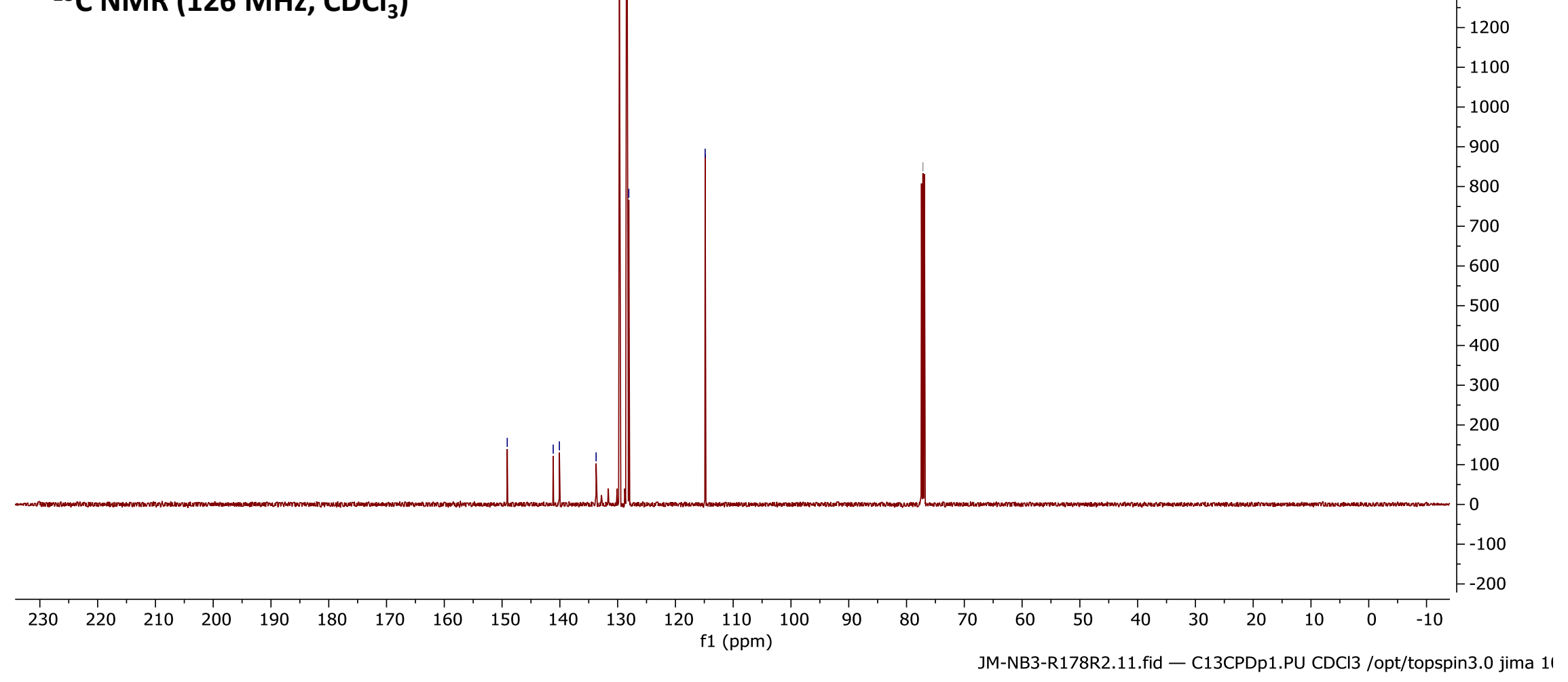




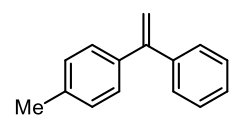

S34

${ }^{1} \mathrm{H}$ NMR (500 MHz, $\mathrm{CDCl}_{3}$ )

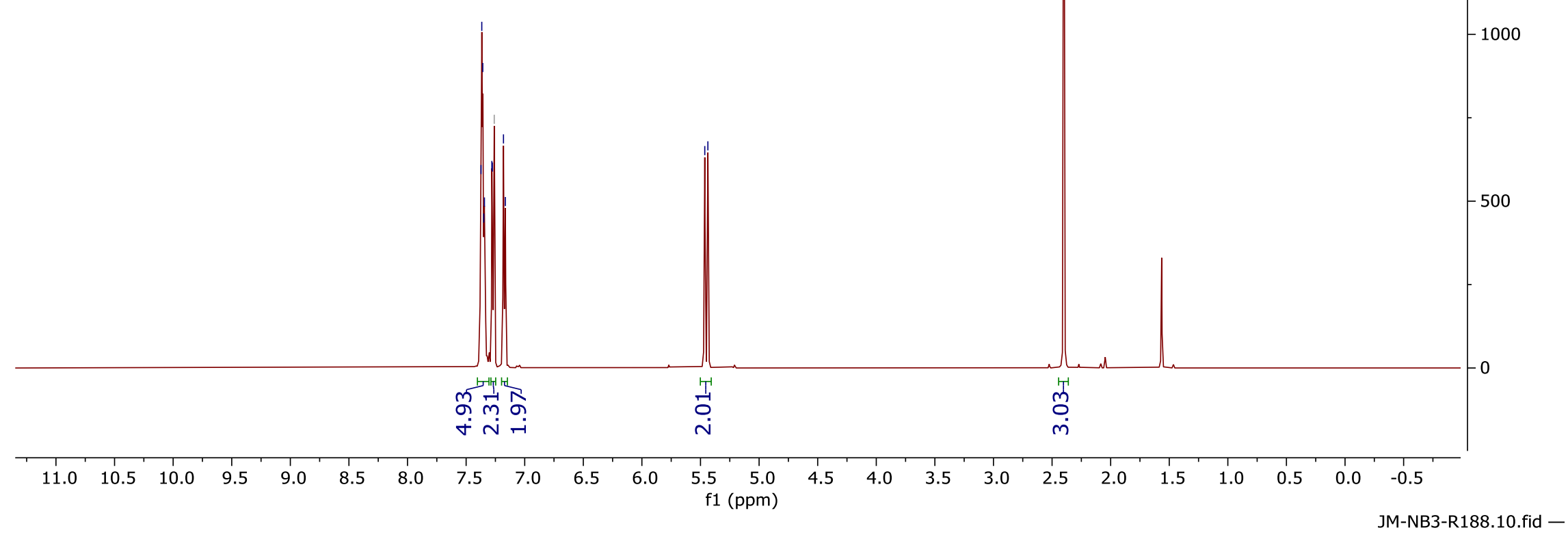




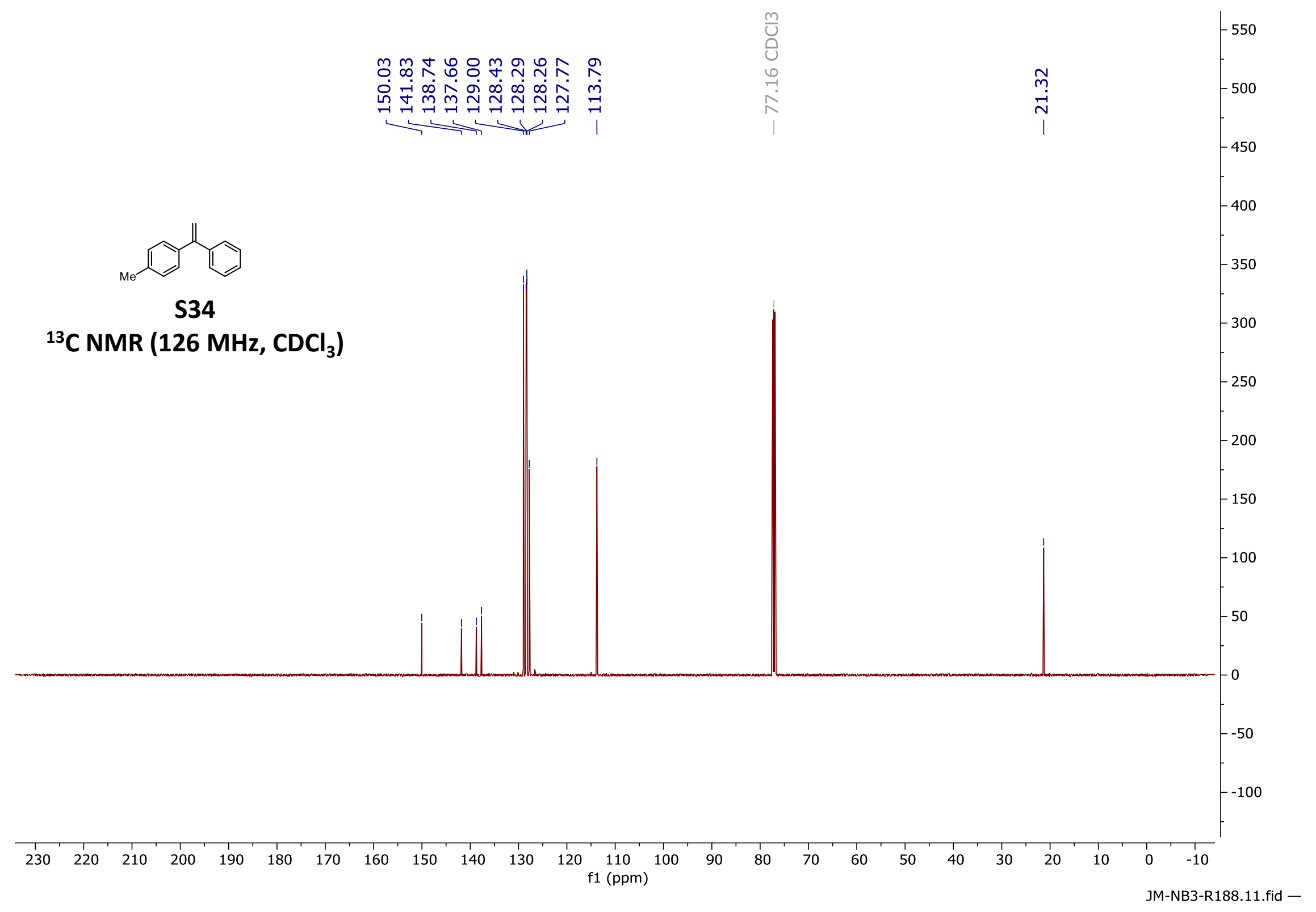




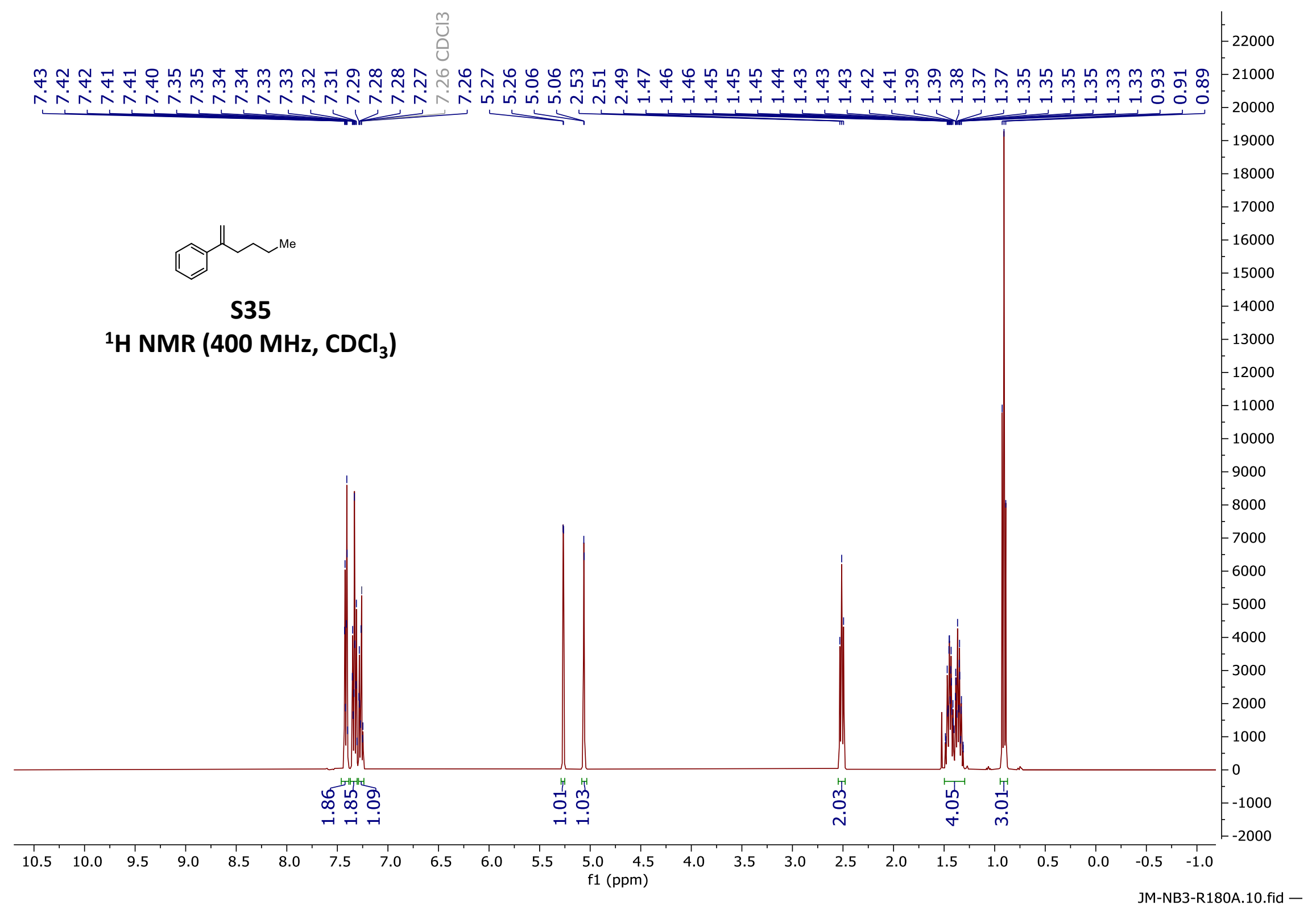




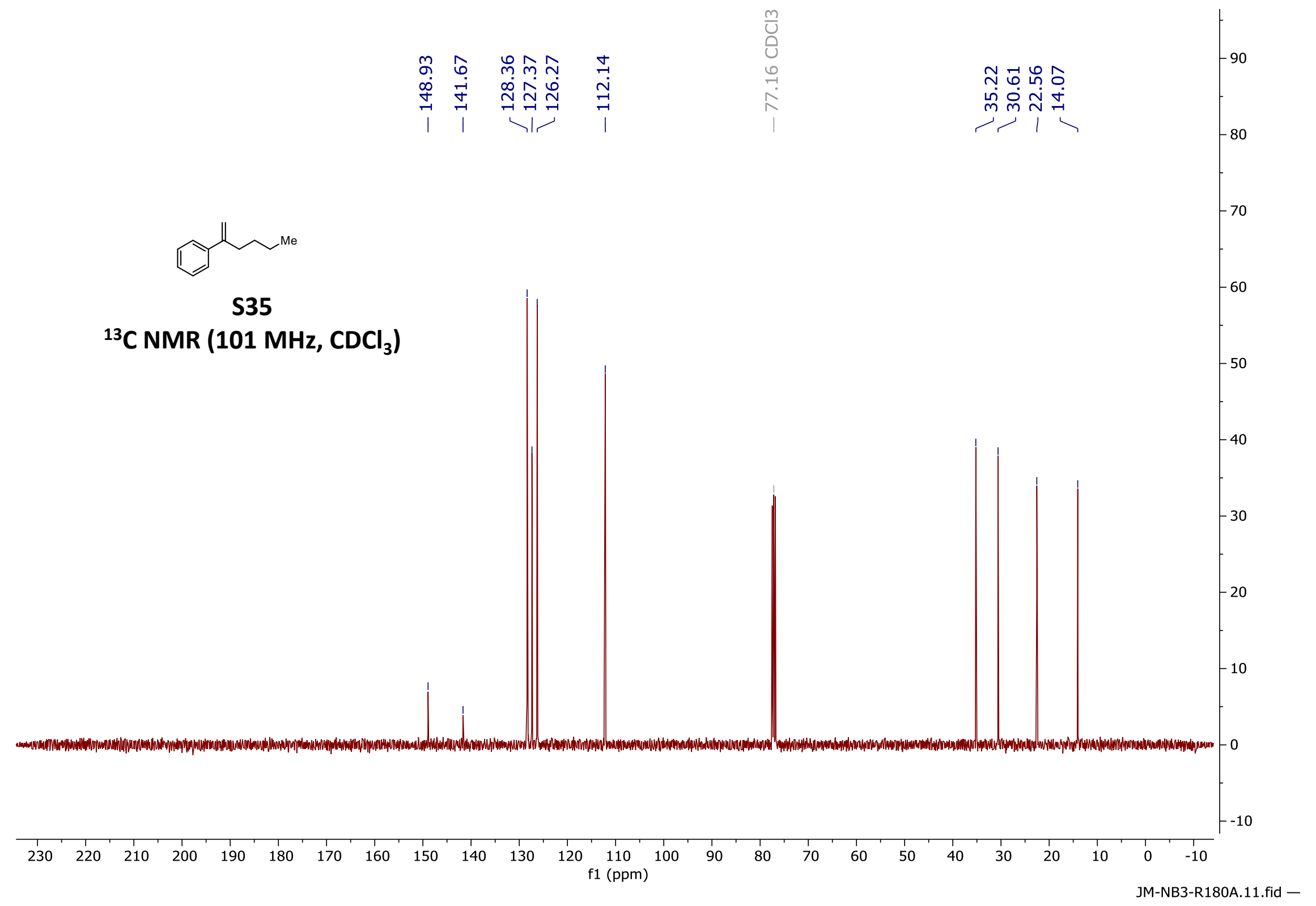




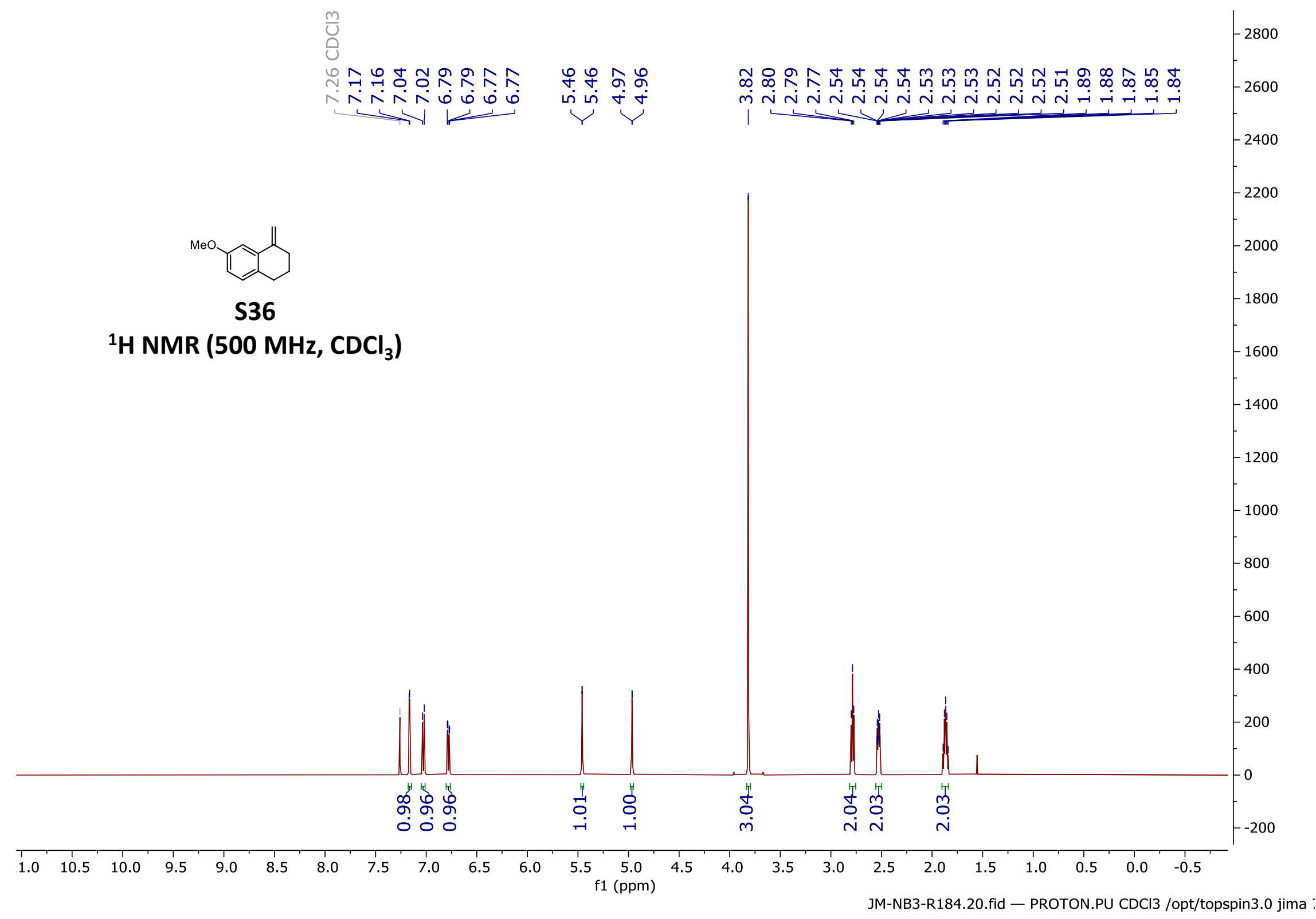




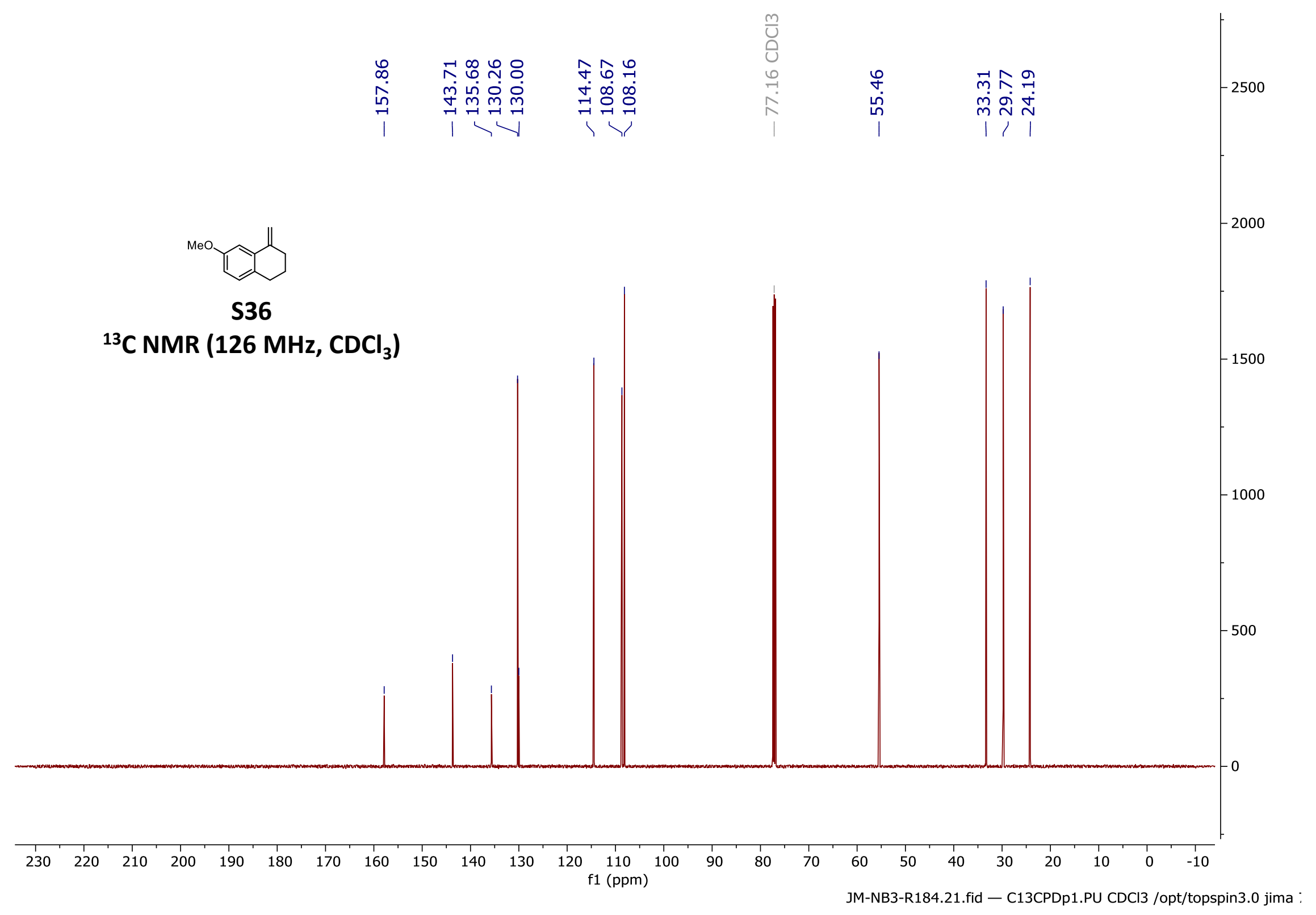




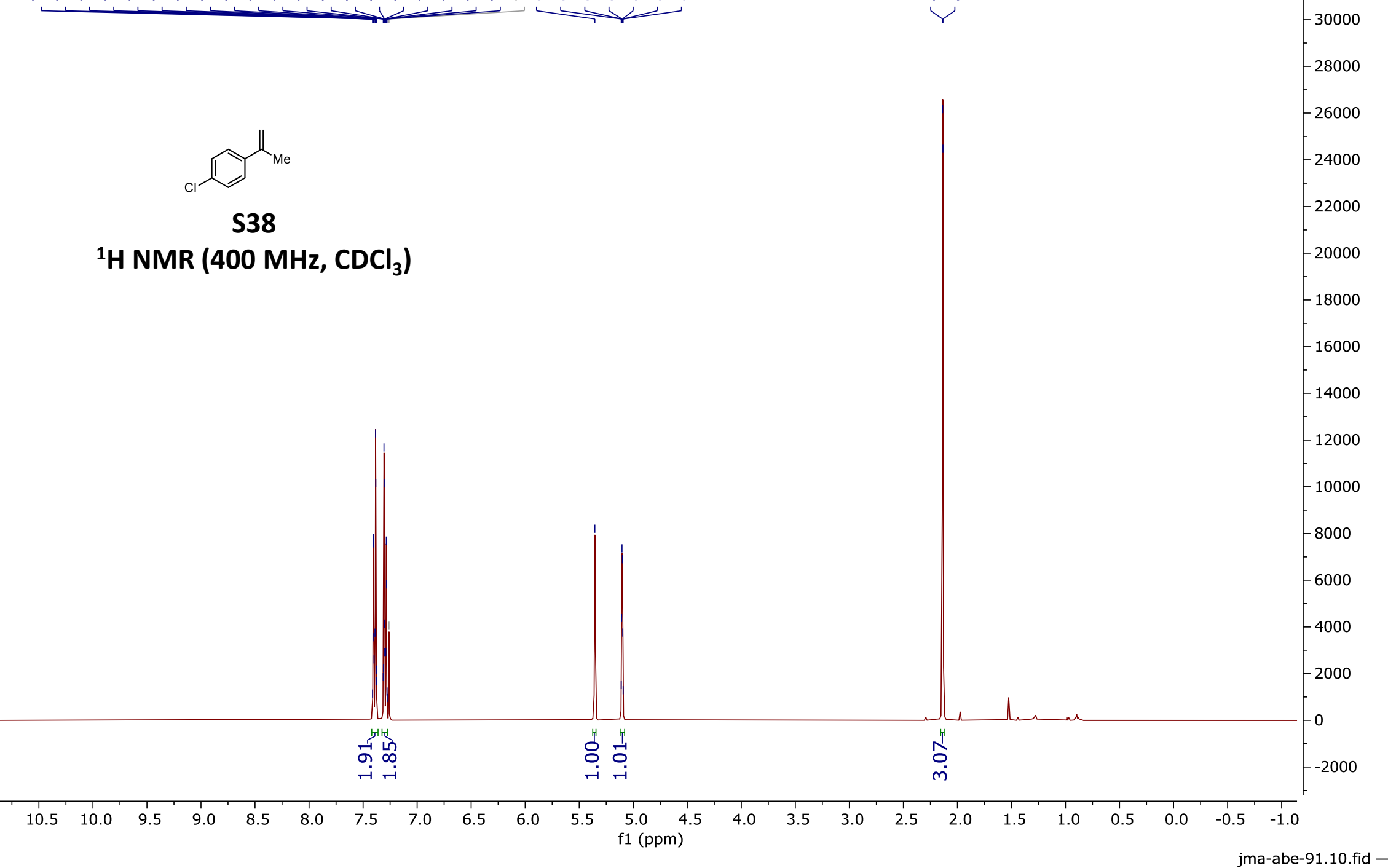




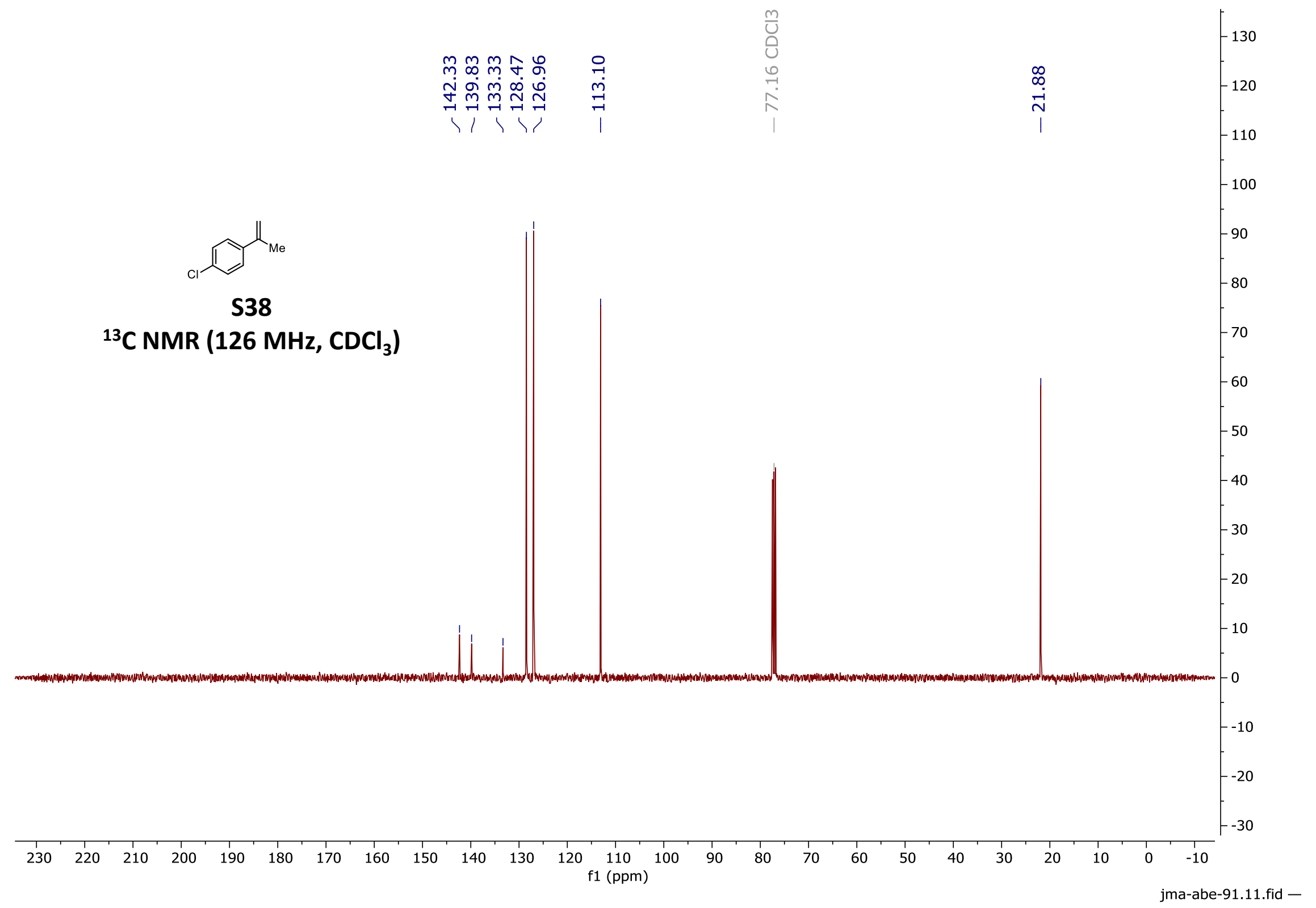




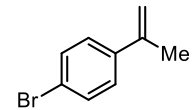

S39

\section{${ }^{1} \mathrm{H}$ NMR (400 MHz, $\mathrm{CDCl}_{3}$ )}

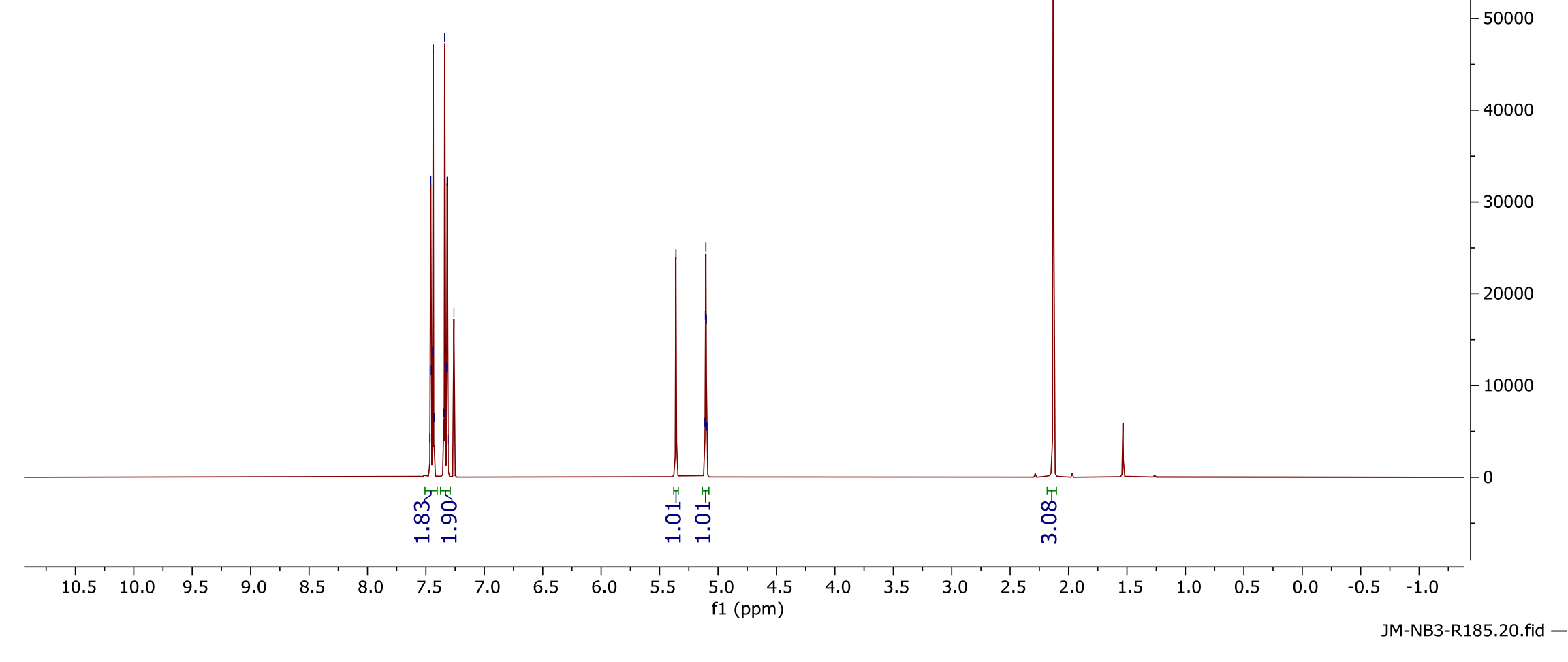




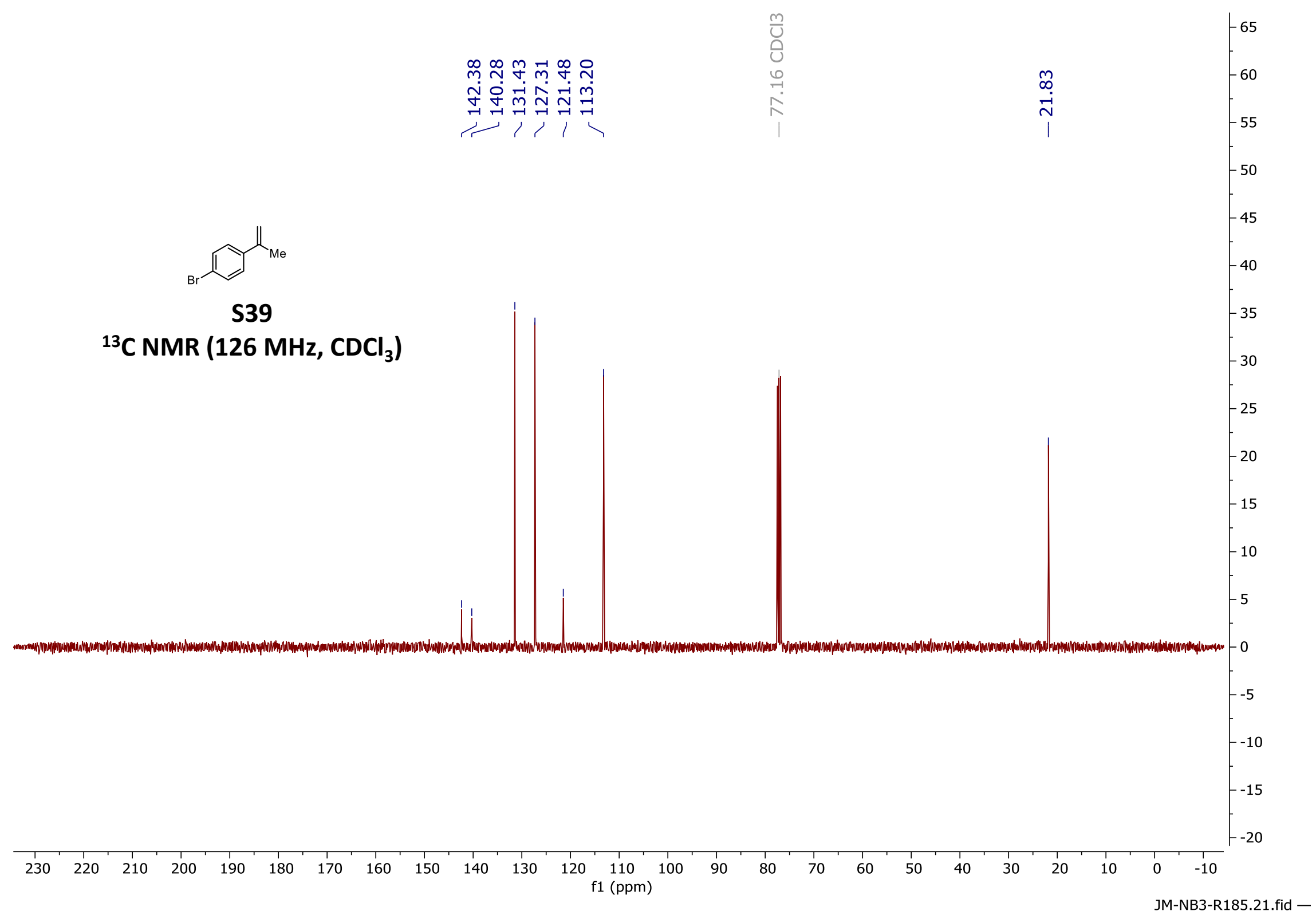




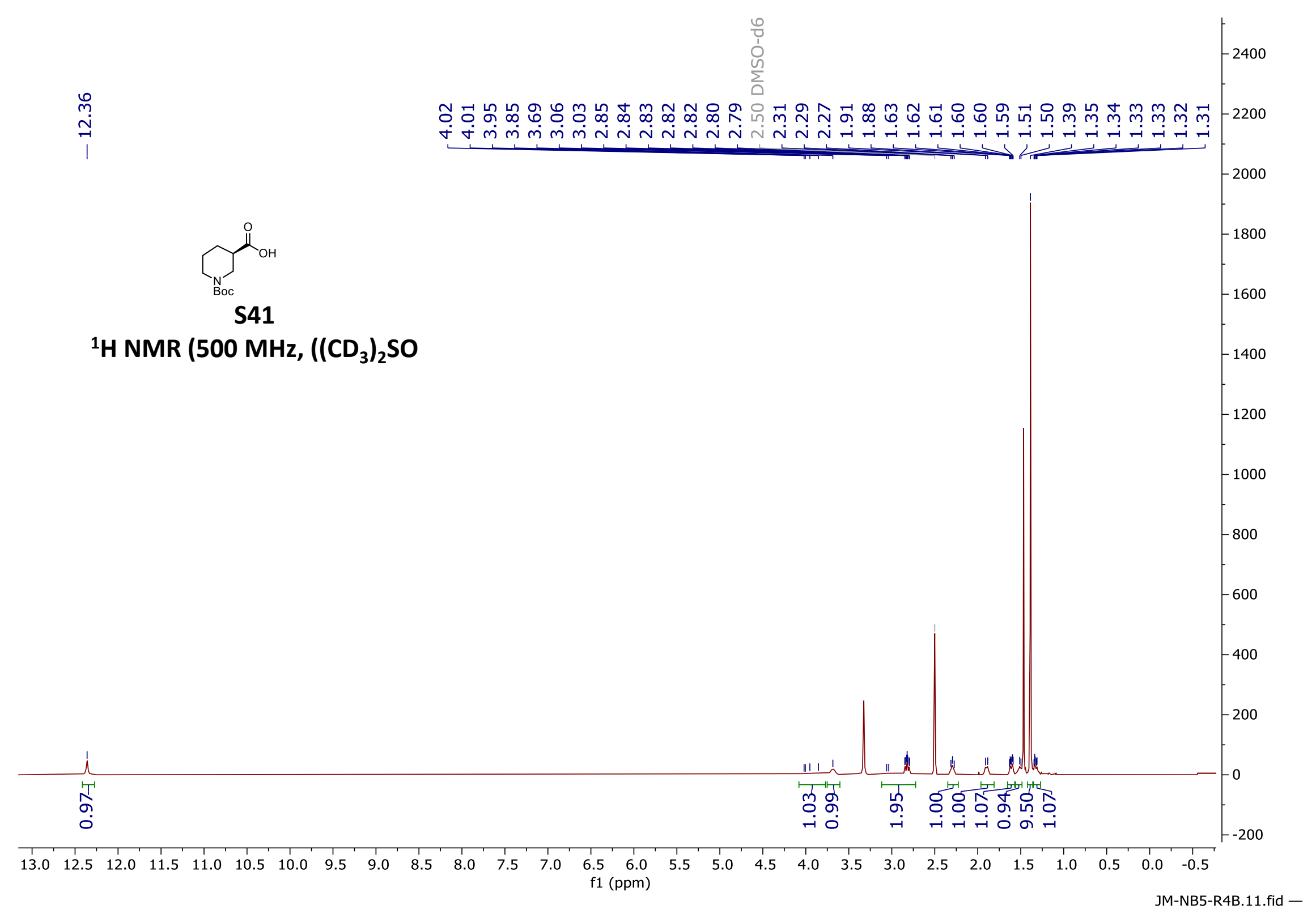




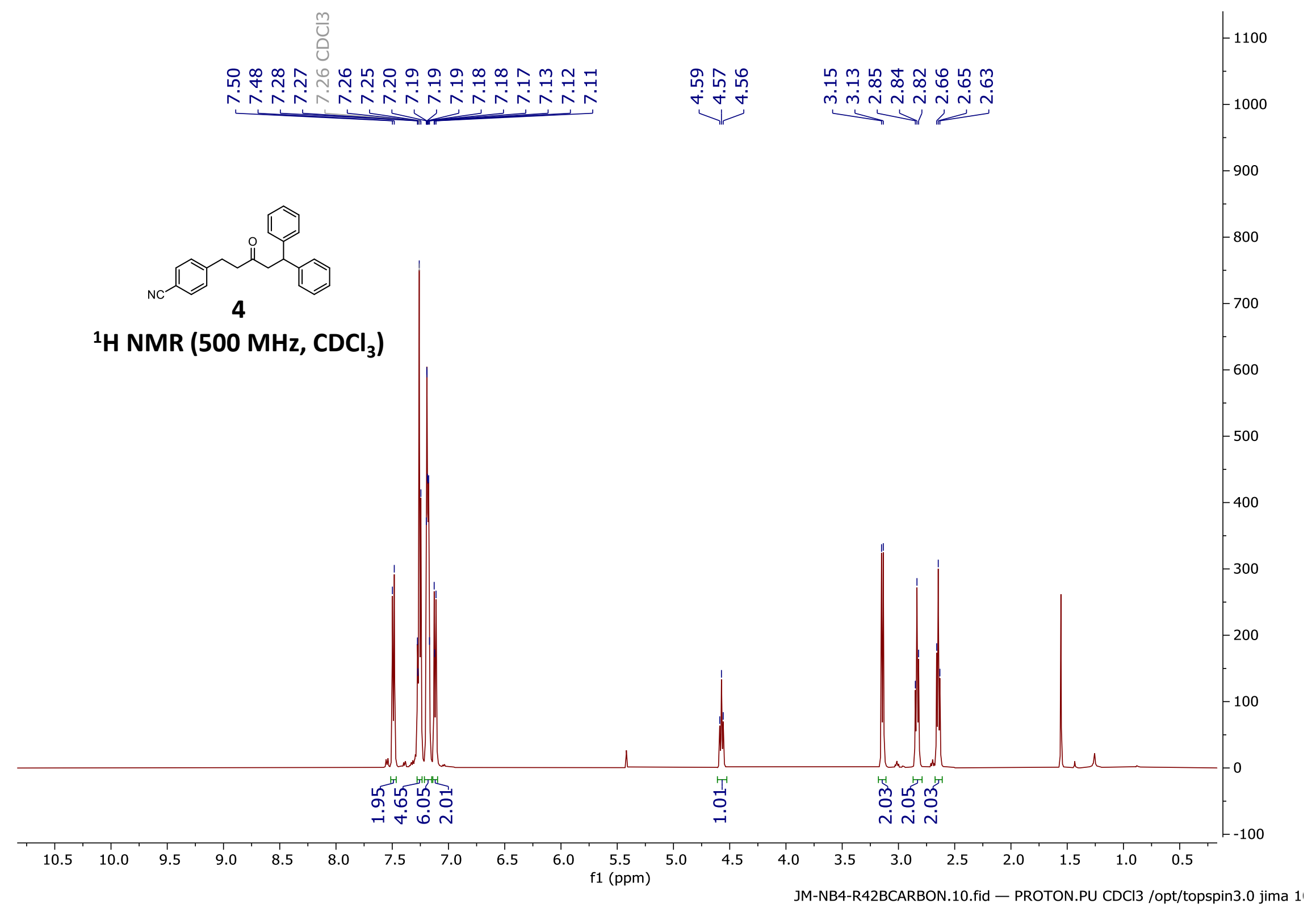




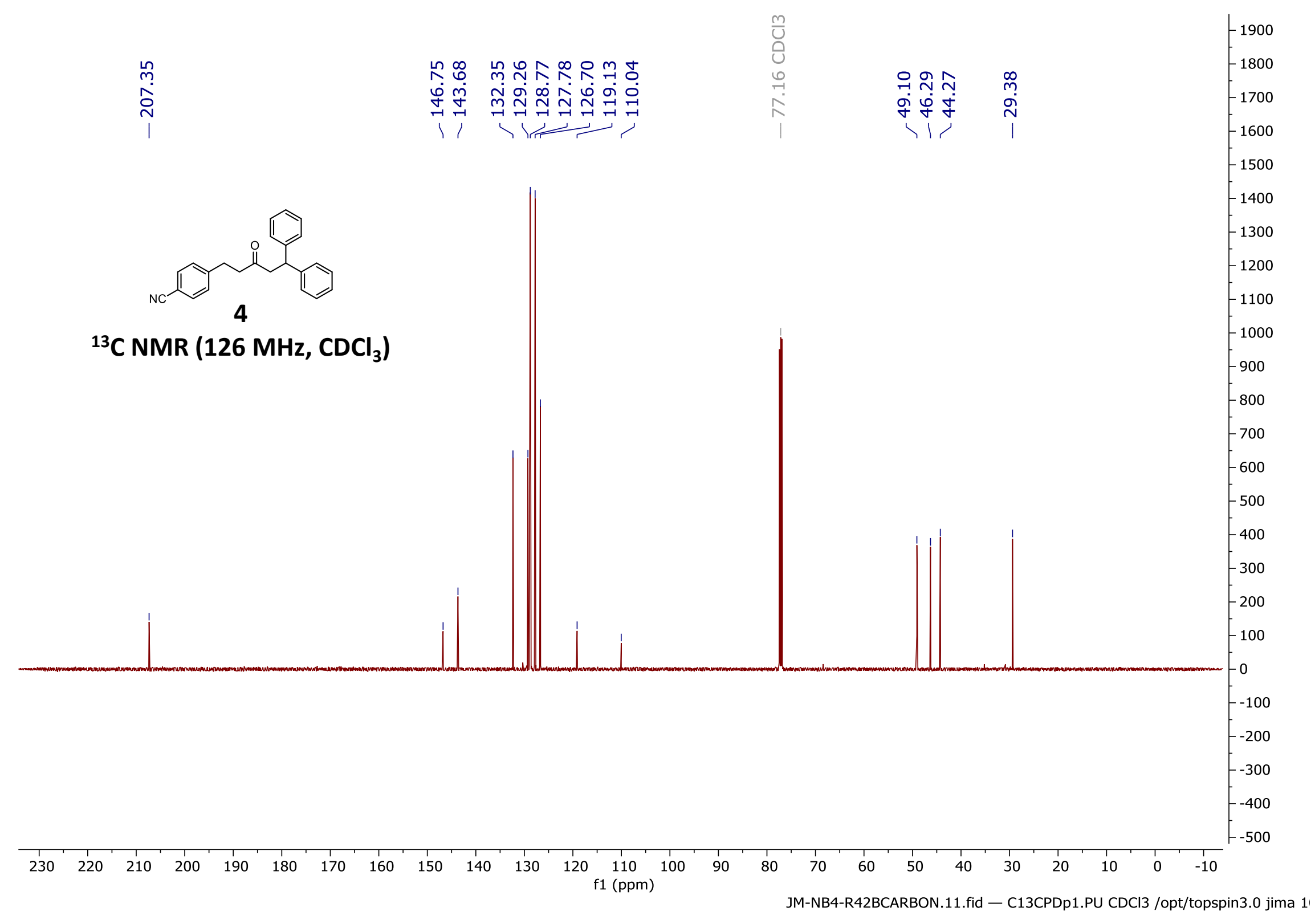




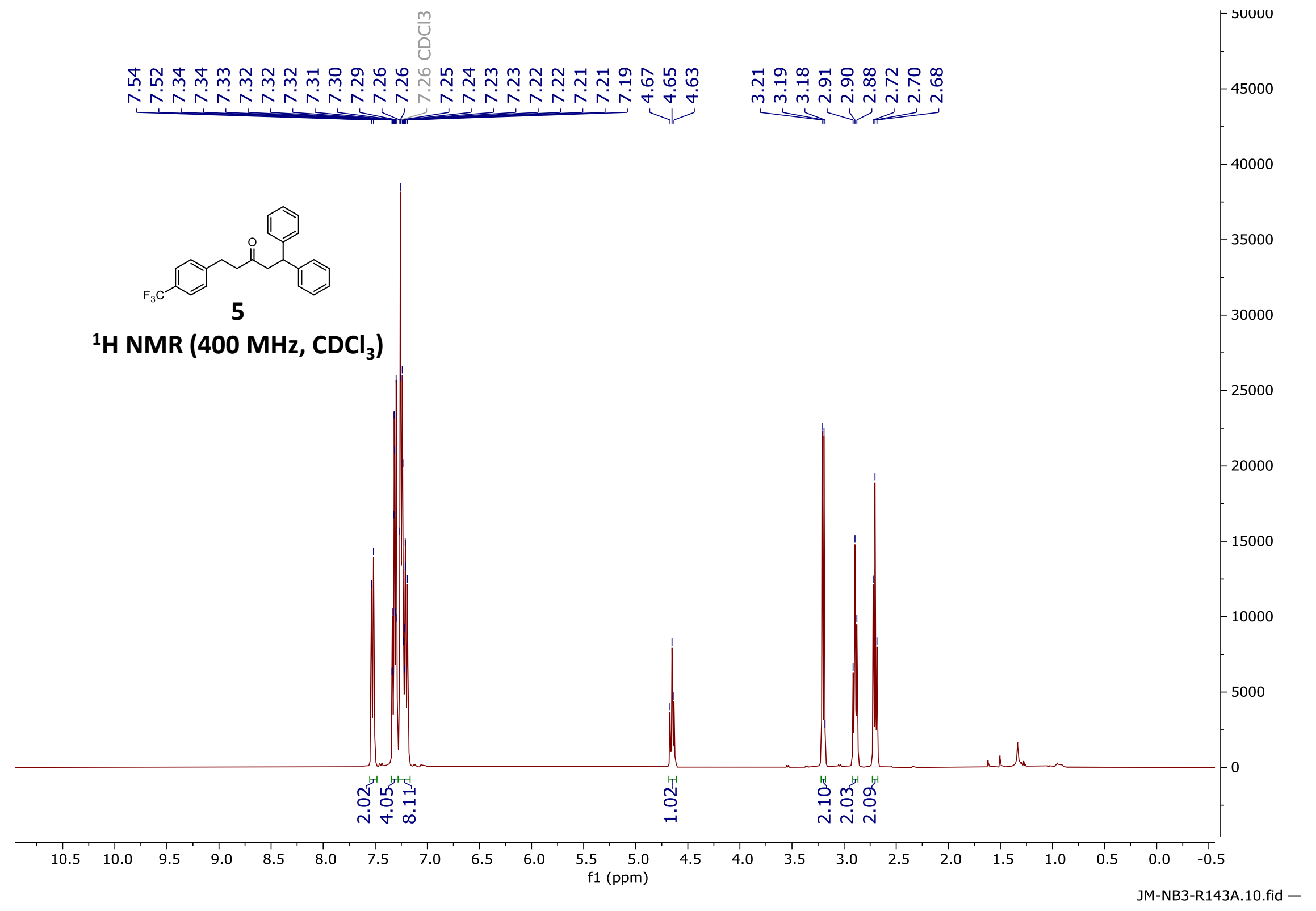




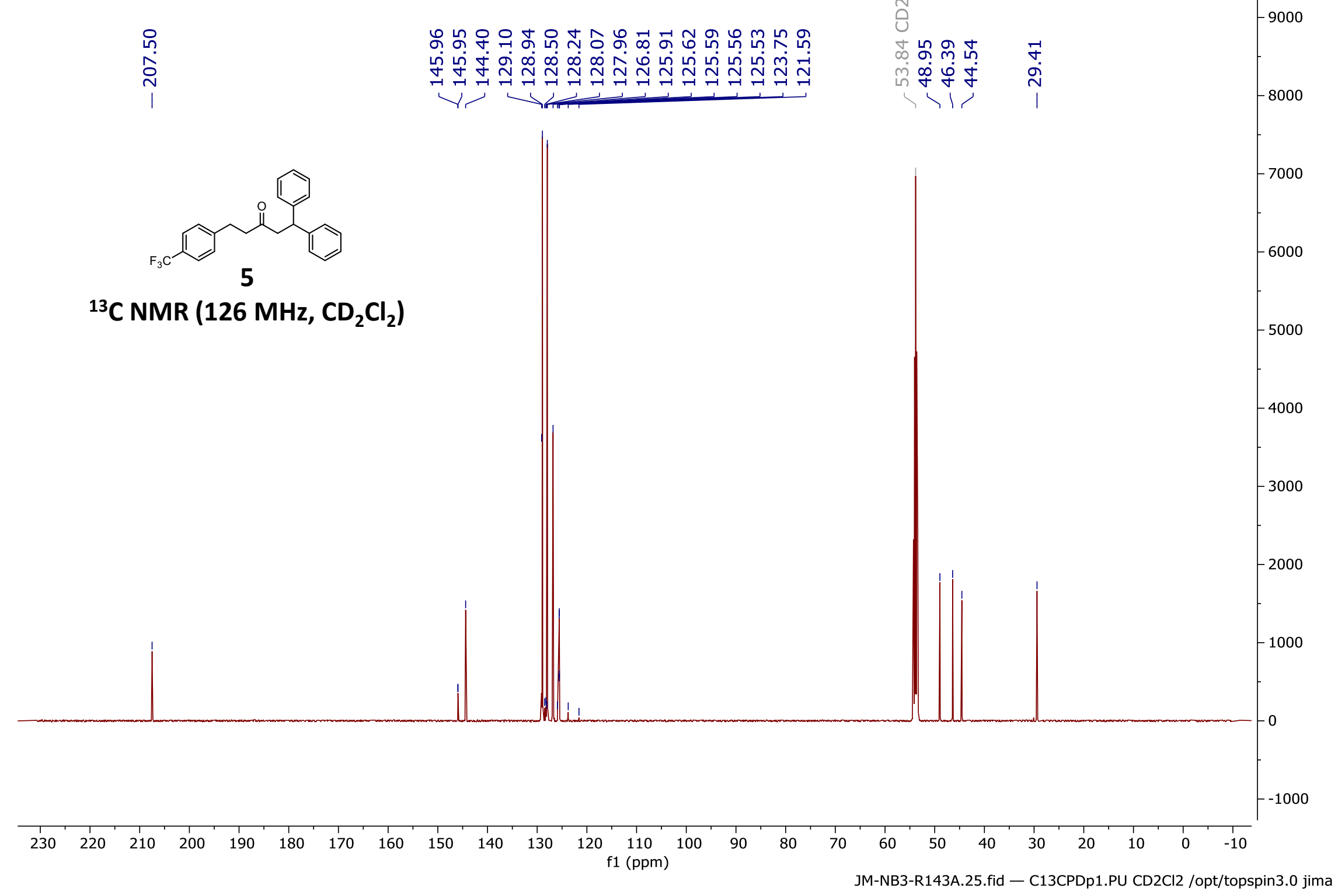




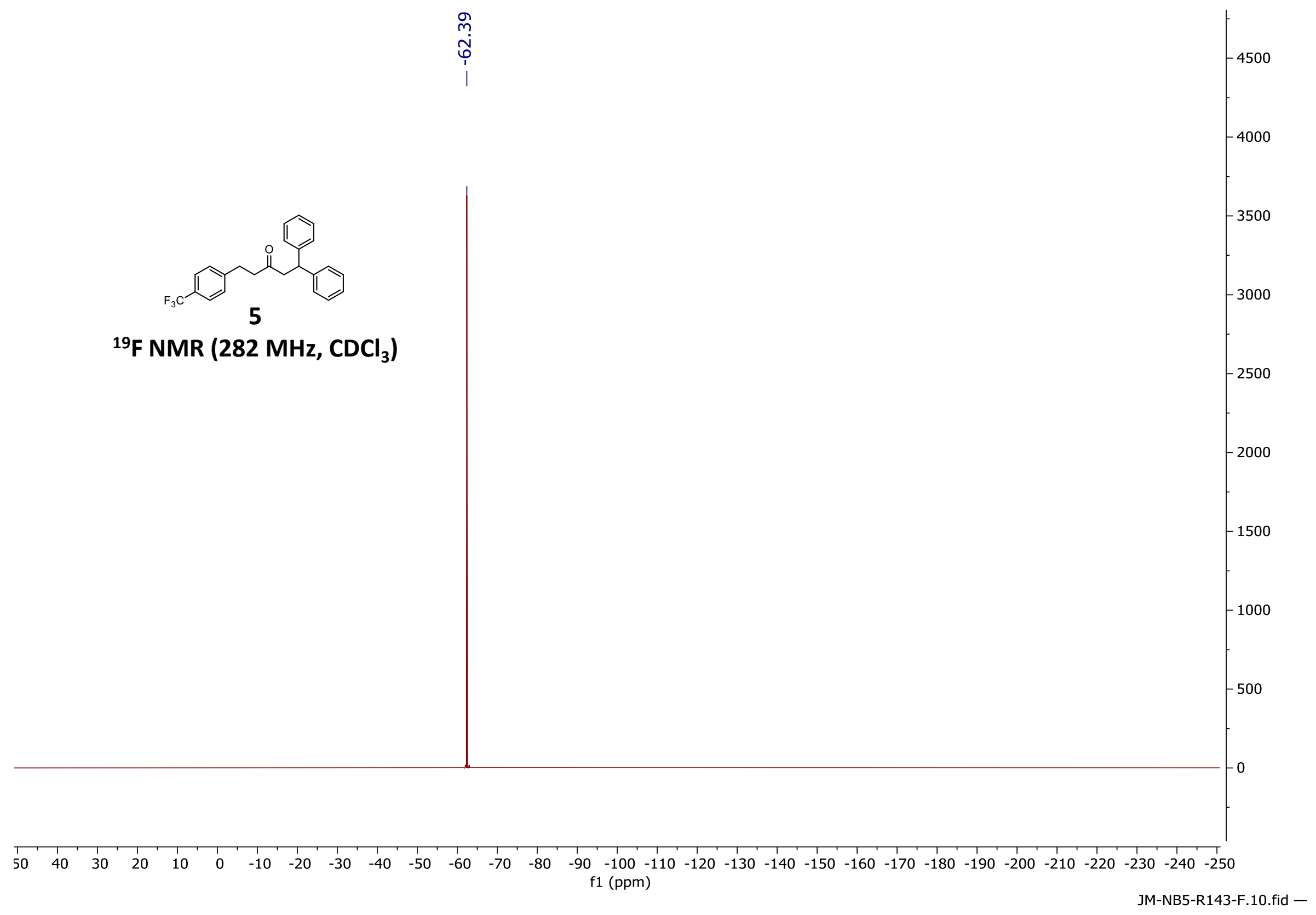




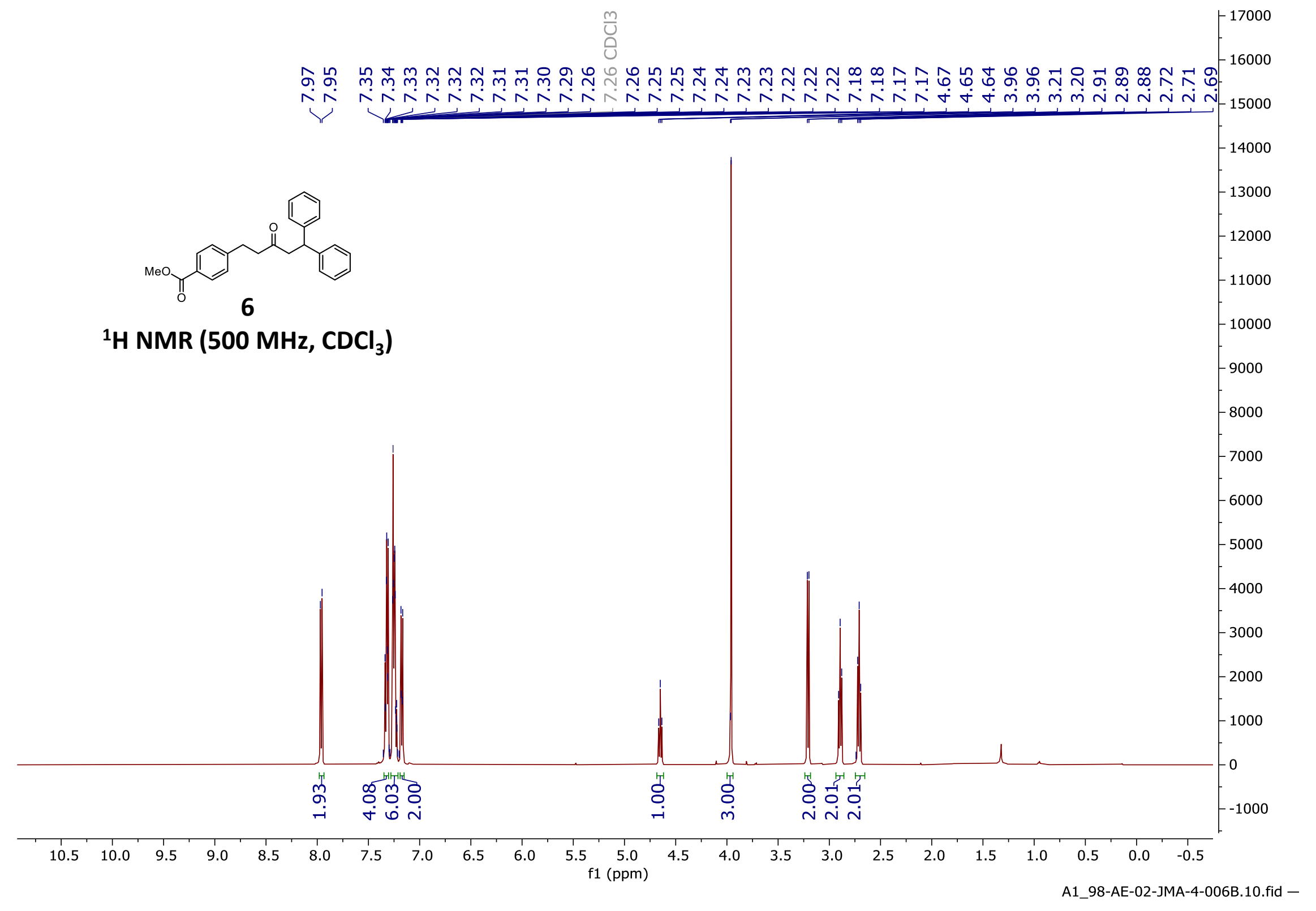




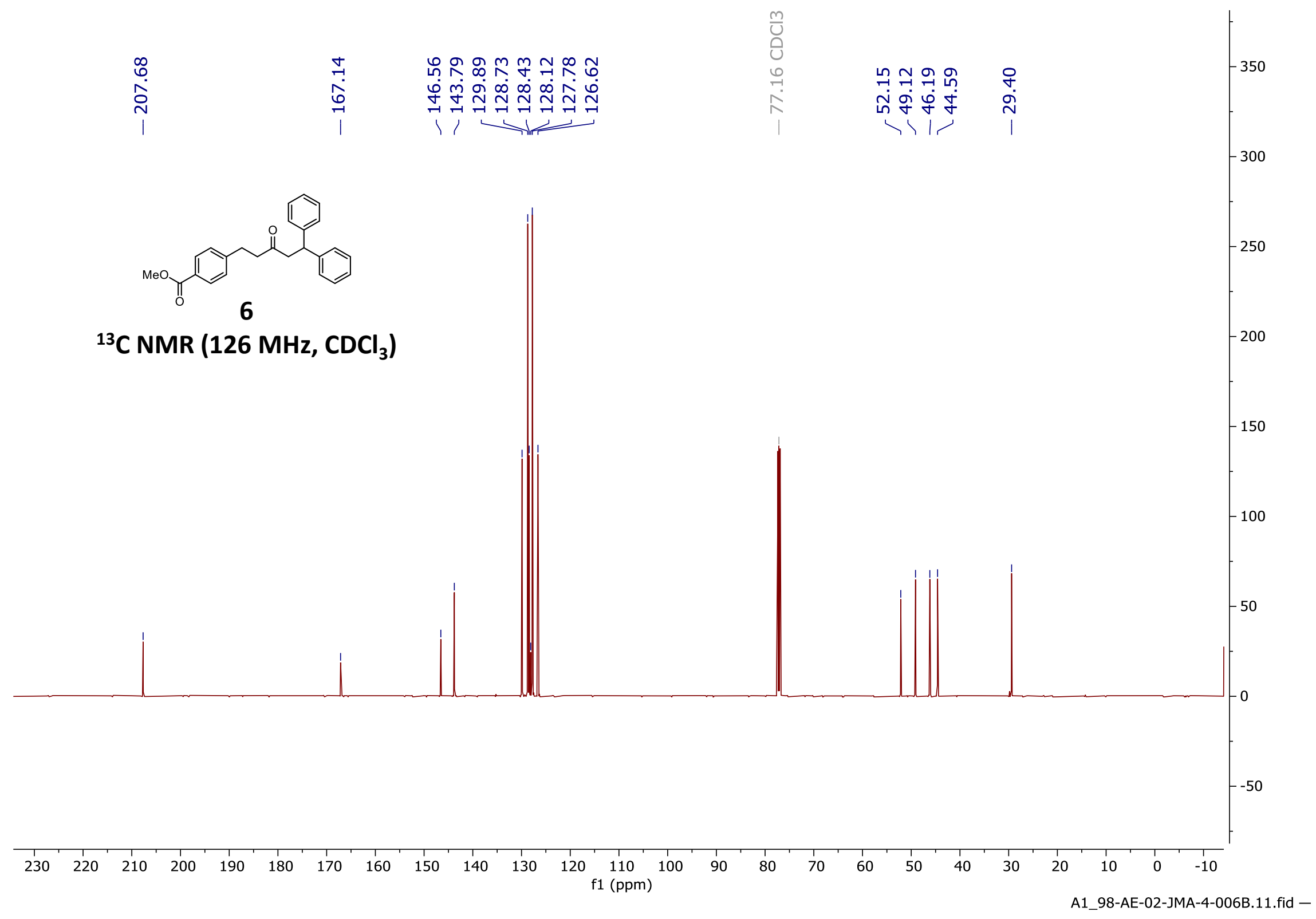




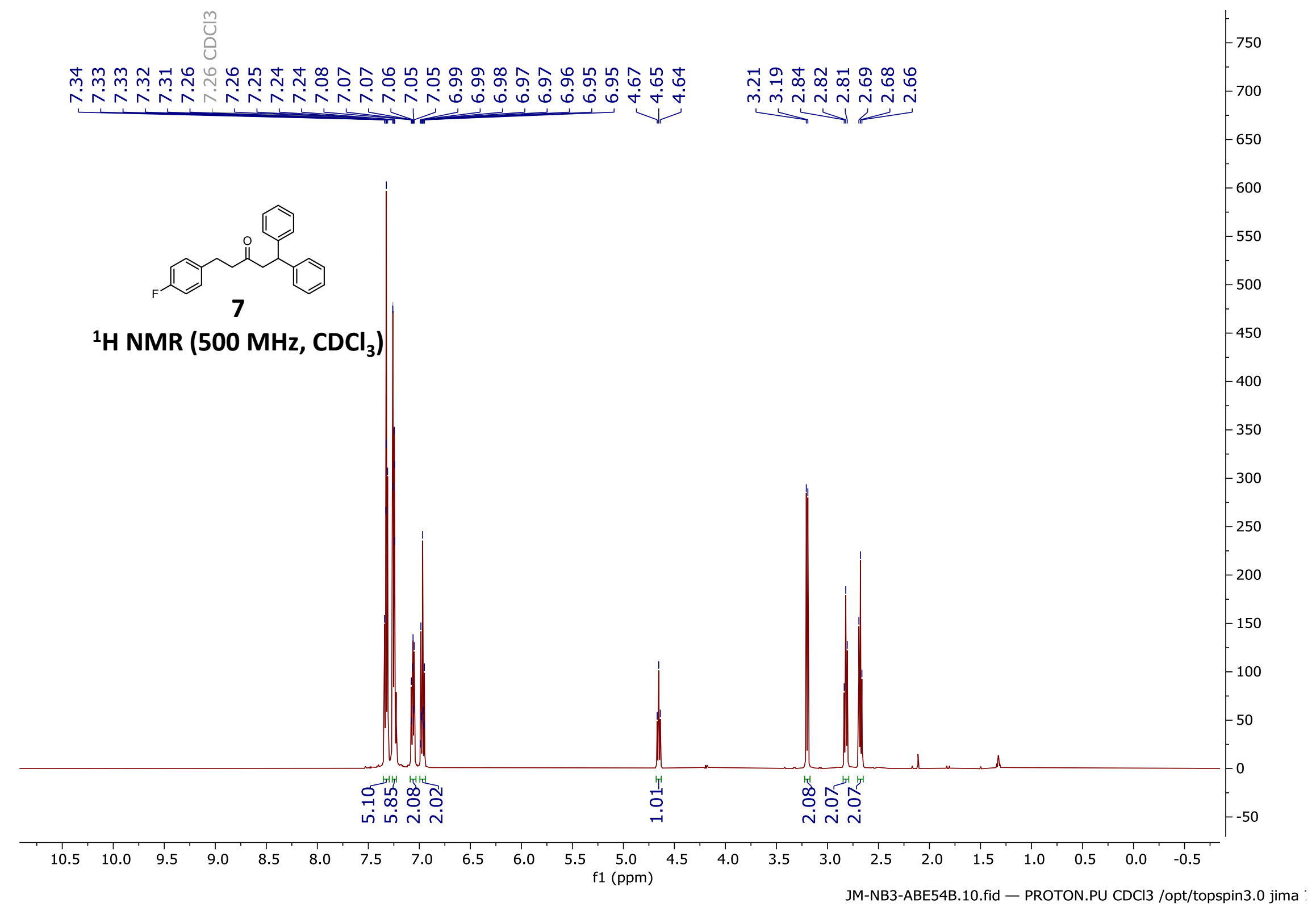




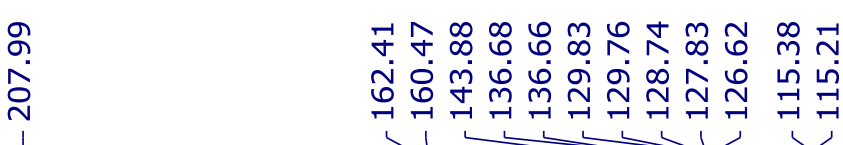

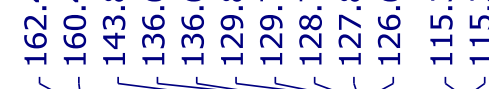

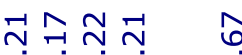

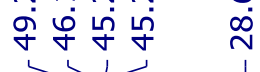

${ }^{13} \mathrm{C} \mathrm{NMR}\left(126 \mathrm{MHz}, \mathrm{CDCl}_{3}\right)$

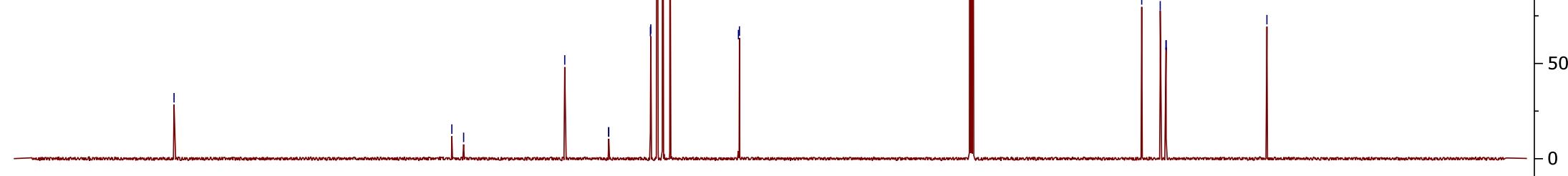




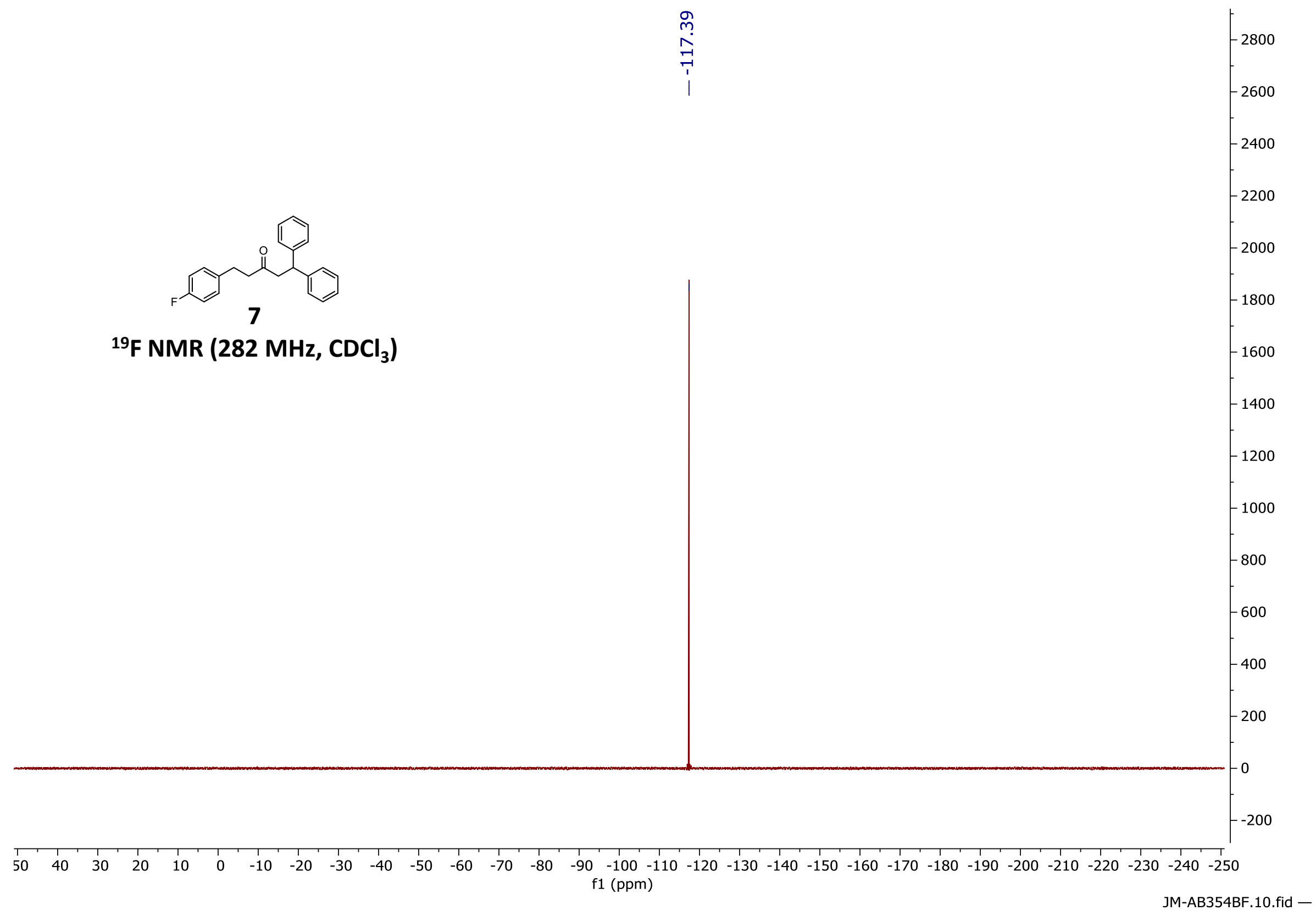




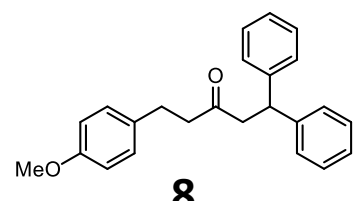

minninnNan

\section{${ }^{1} \mathrm{H}$ NMR $\left(500 \mathrm{MHz}, \mathrm{CDCl}_{3}\right)$}

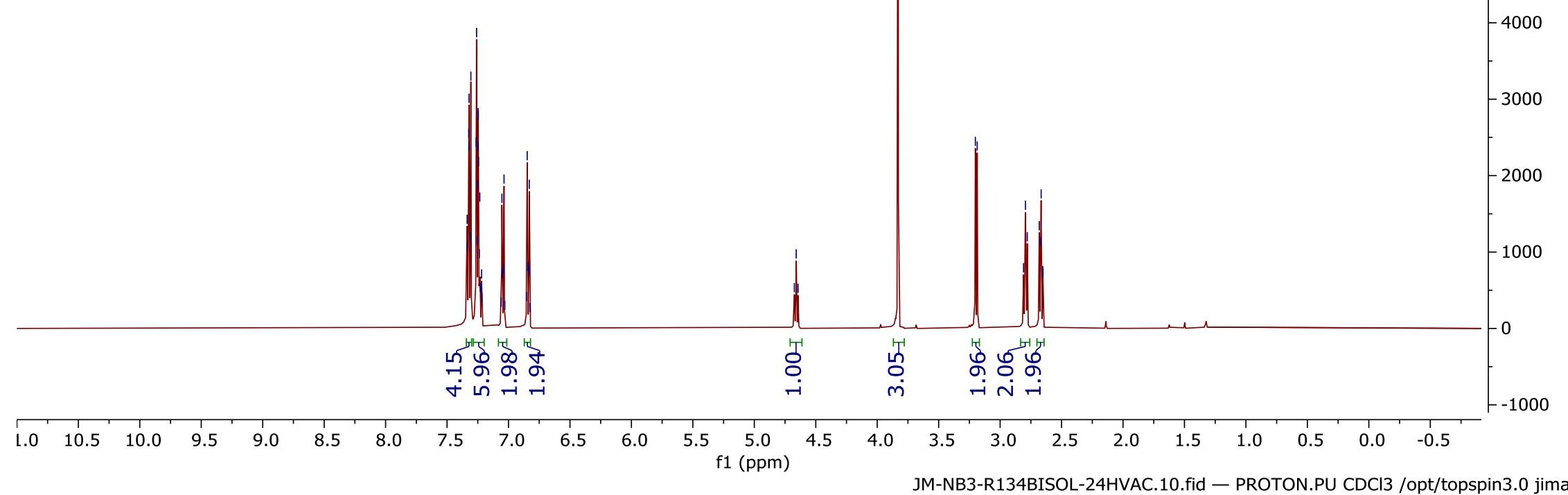




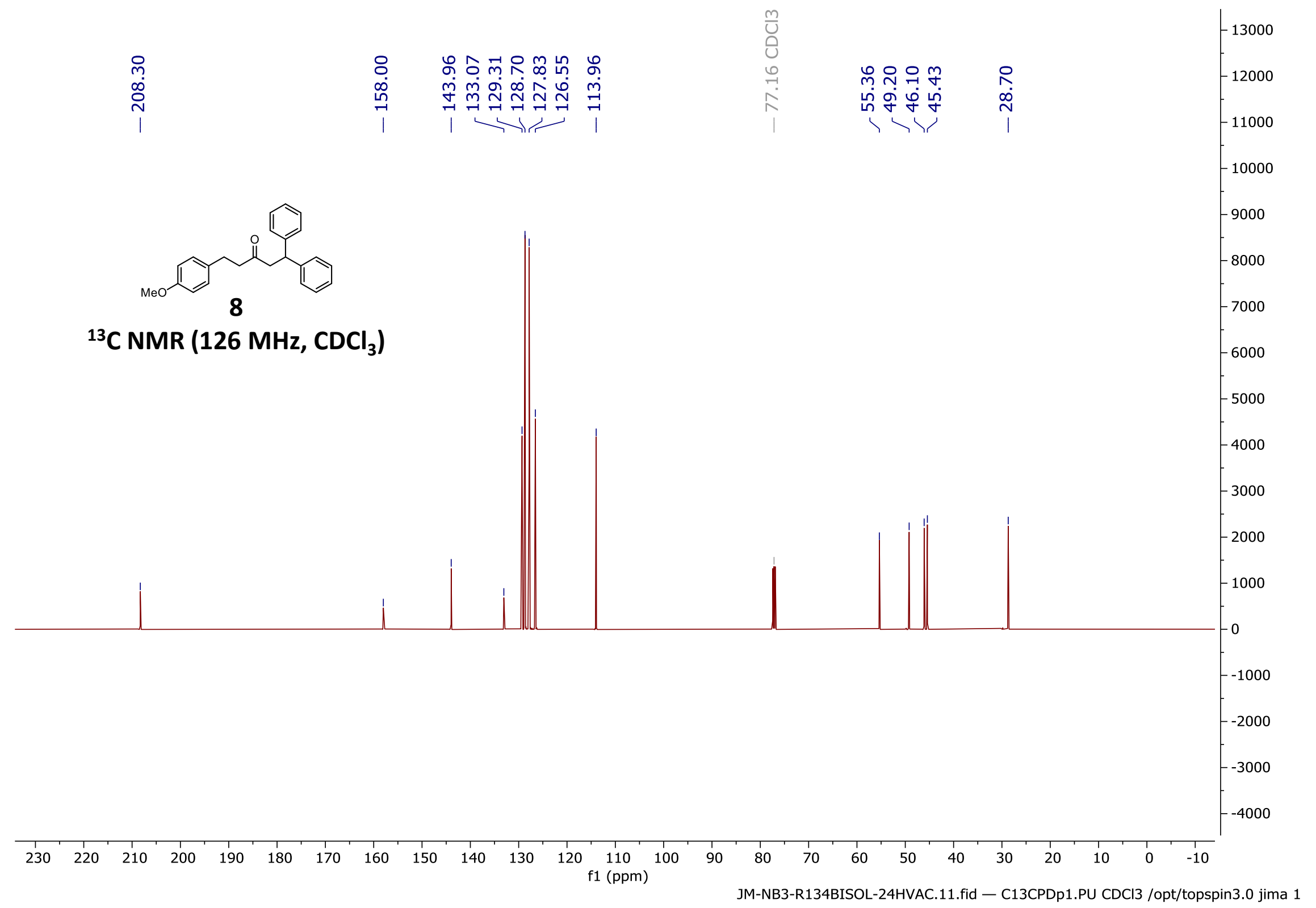




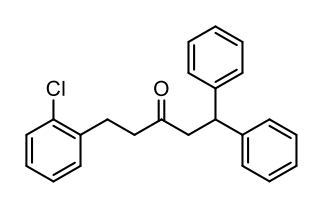

9

${ }^{1} \mathrm{H}$ NMR $\left(500 \mathrm{MHz}, \mathrm{CDCl}_{3}\right)$

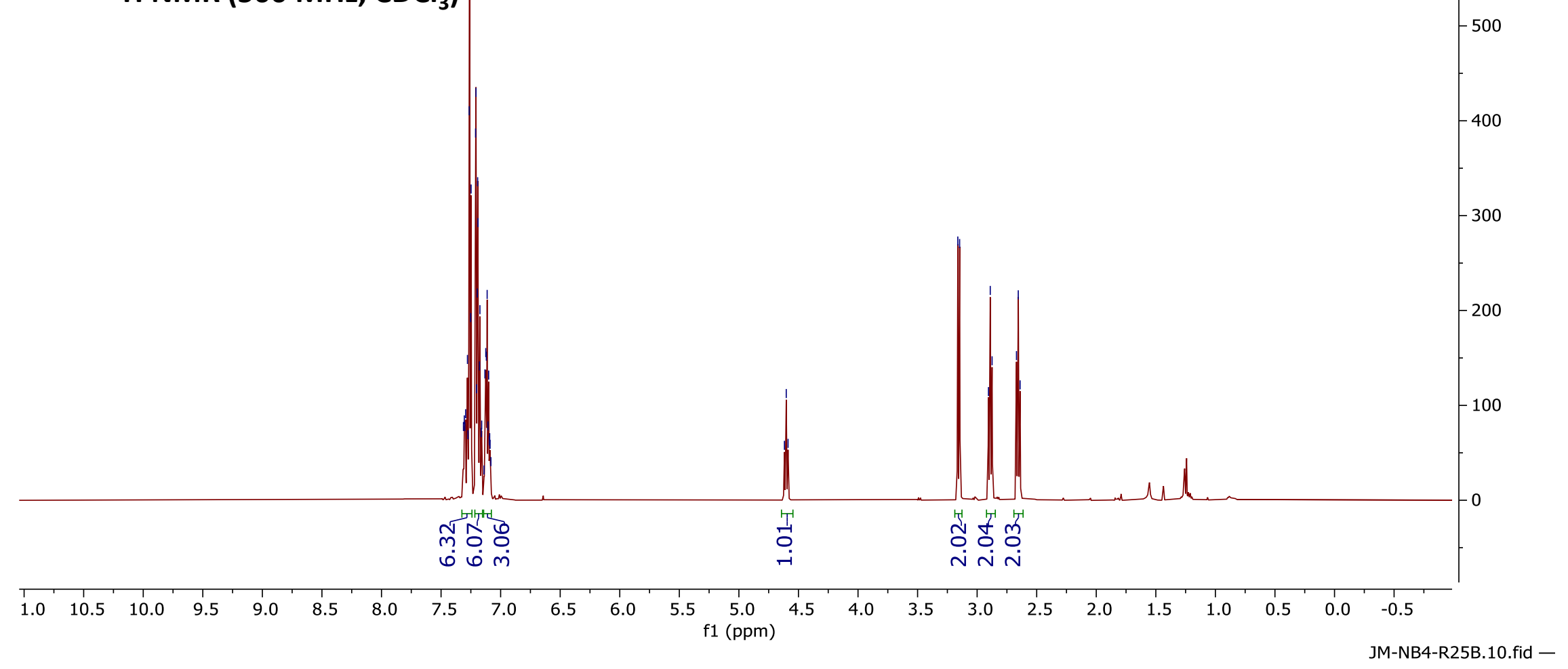




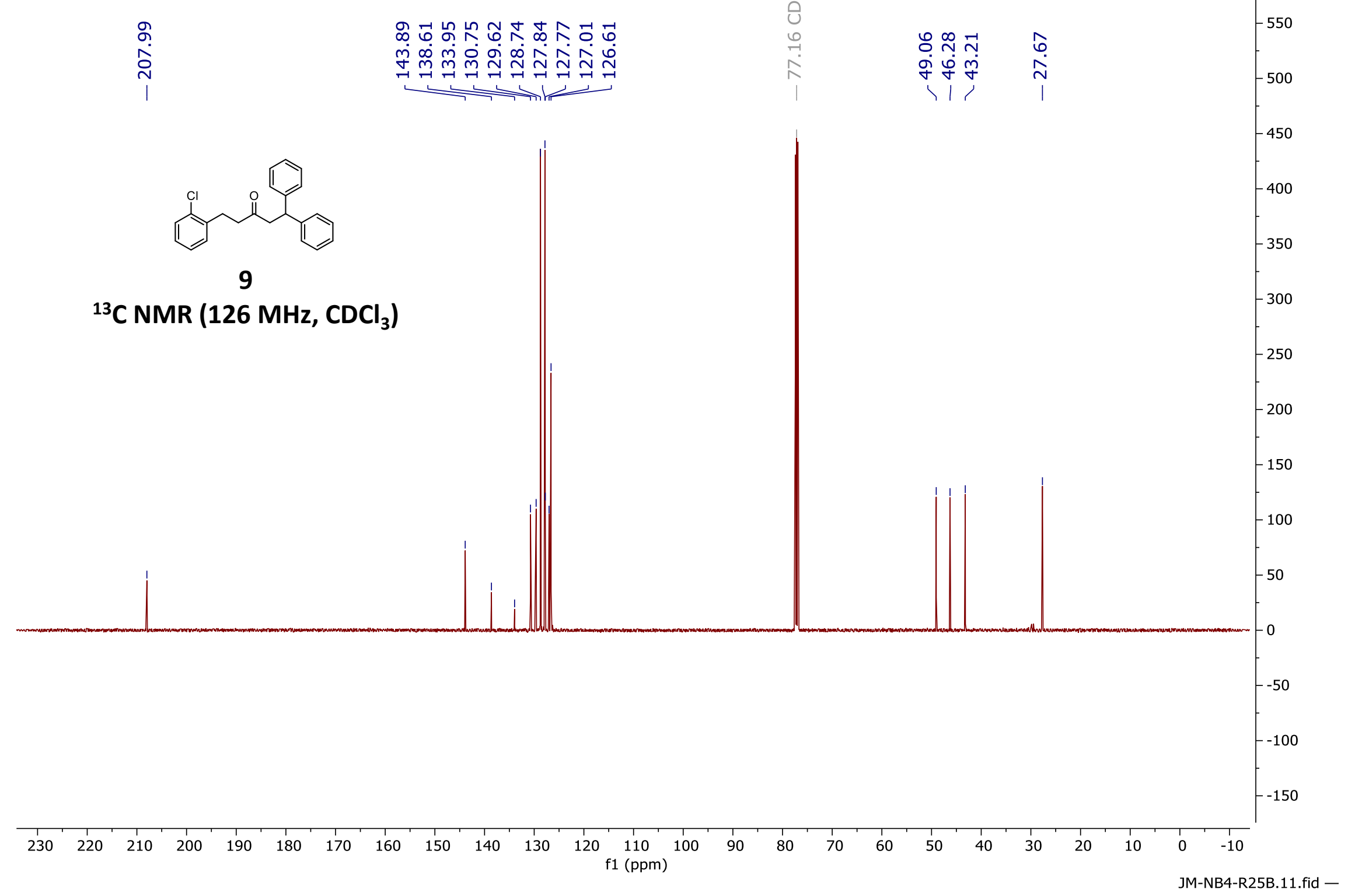




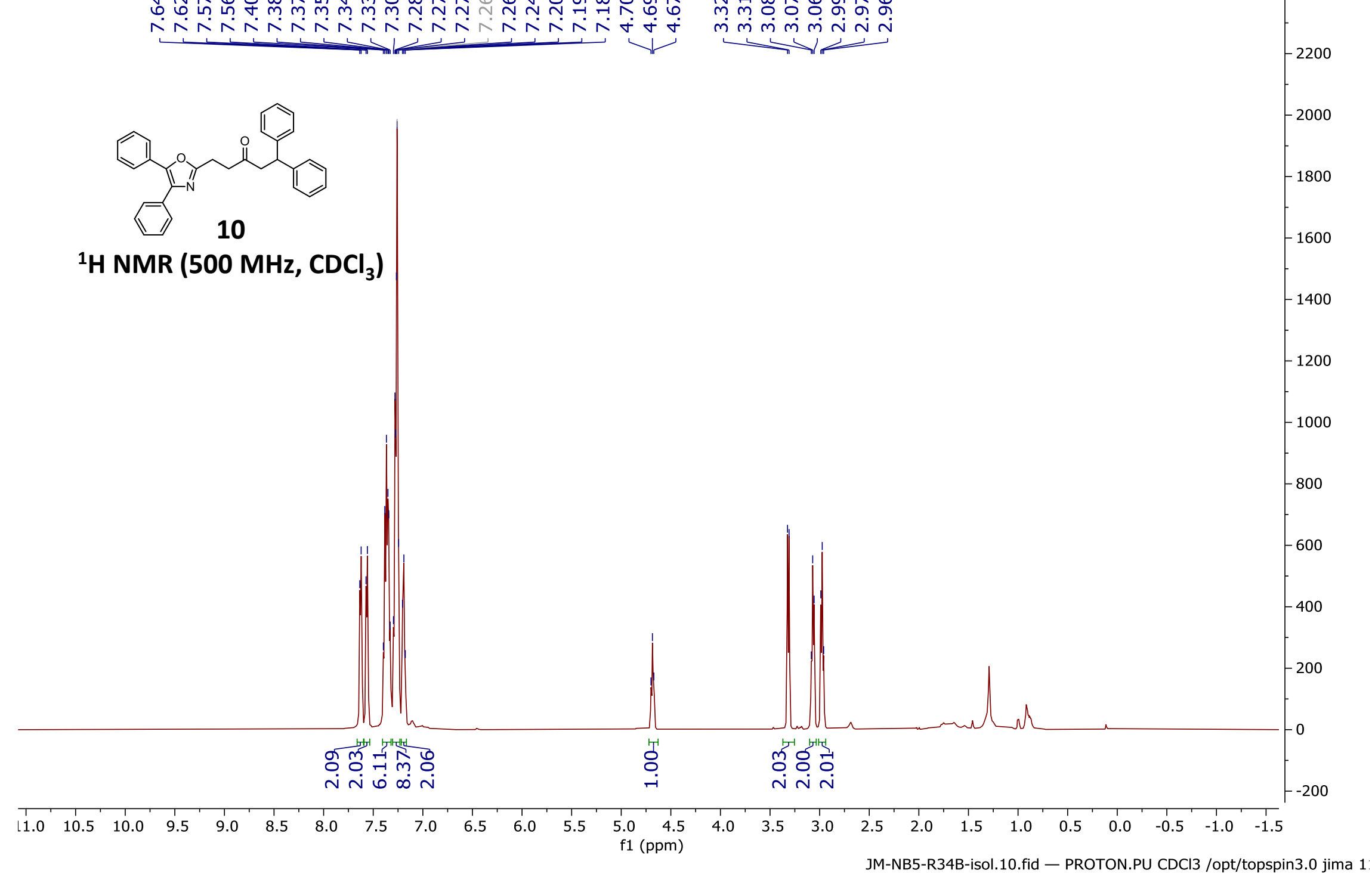




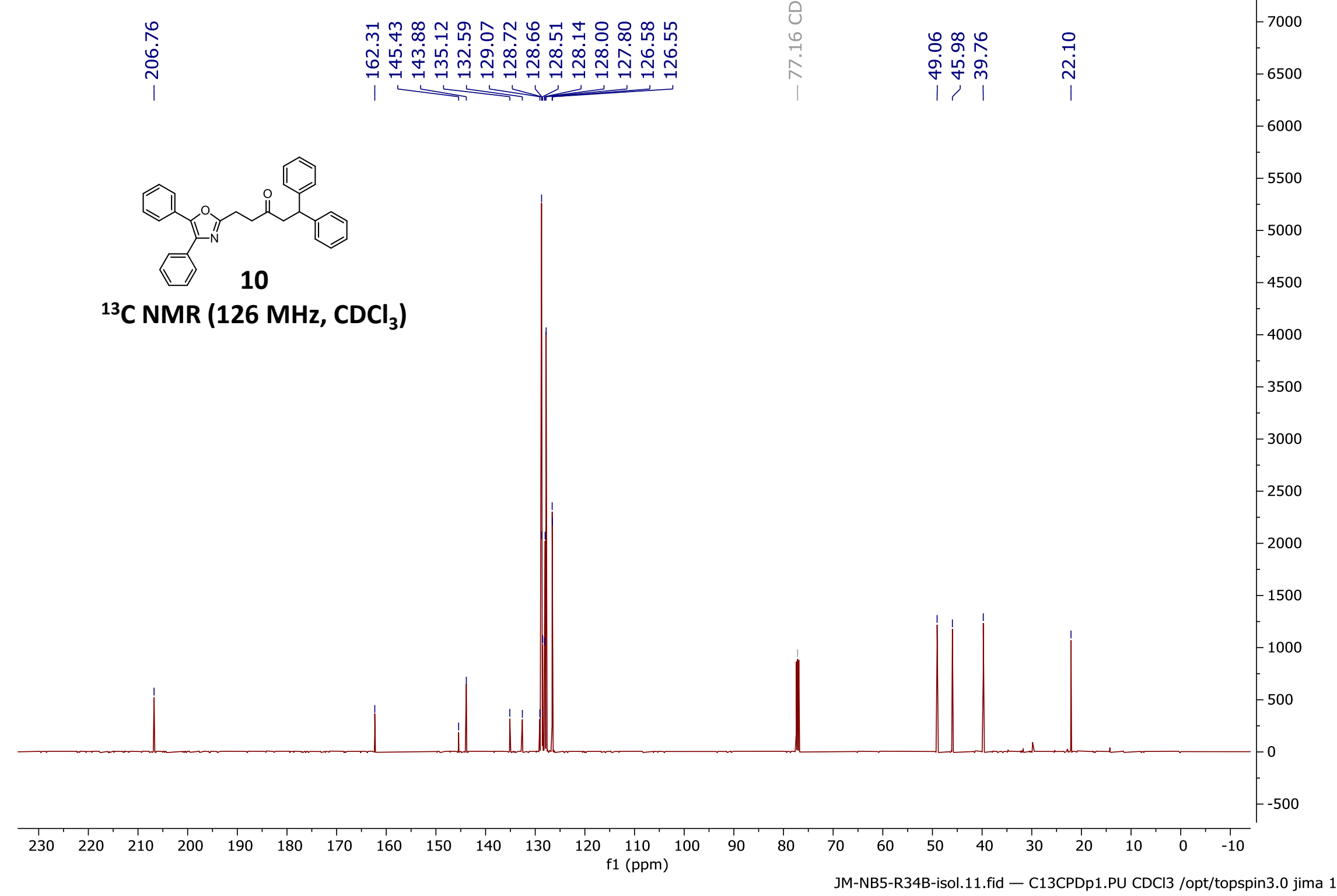




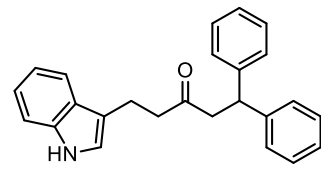

11

${ }^{1} \mathrm{H}$ NMR $\left(500 \mathrm{MHz}, \mathrm{CDCl}_{3}\right)$

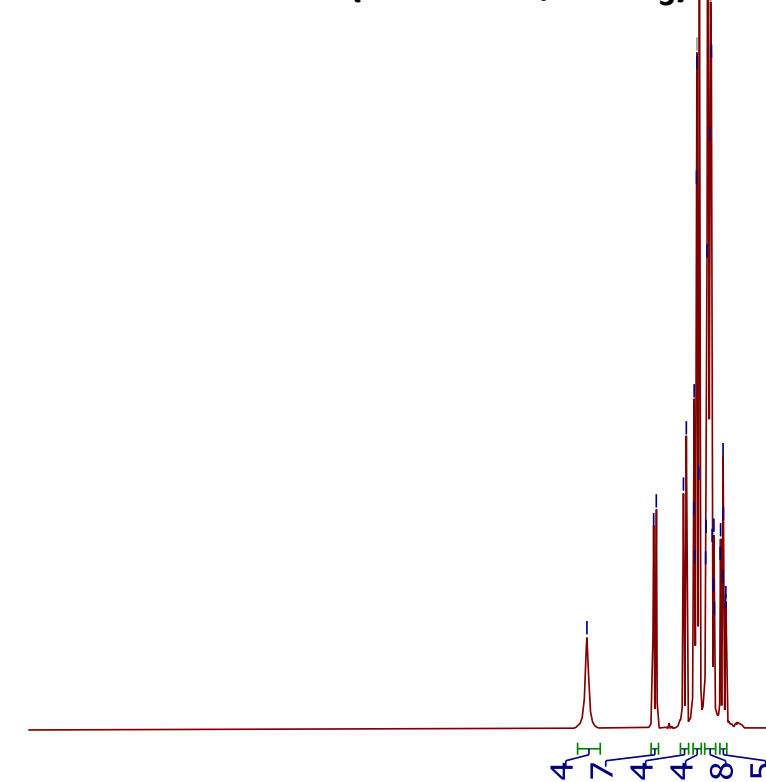

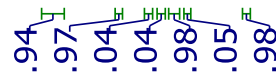

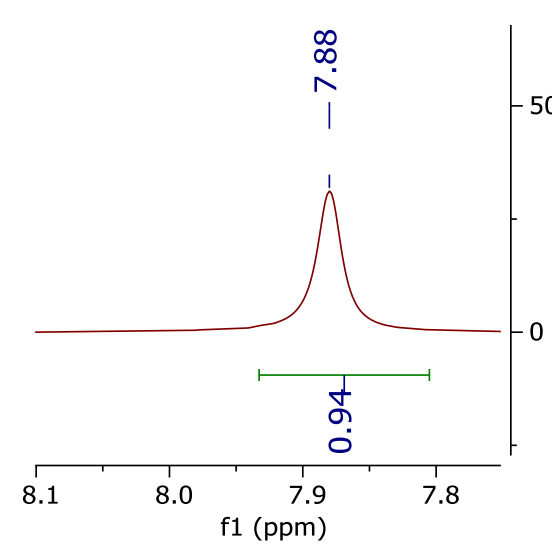

$-500$

$-3000$

$-2500$

$-2000$

$-1500$

$-1000$

$-500$ 


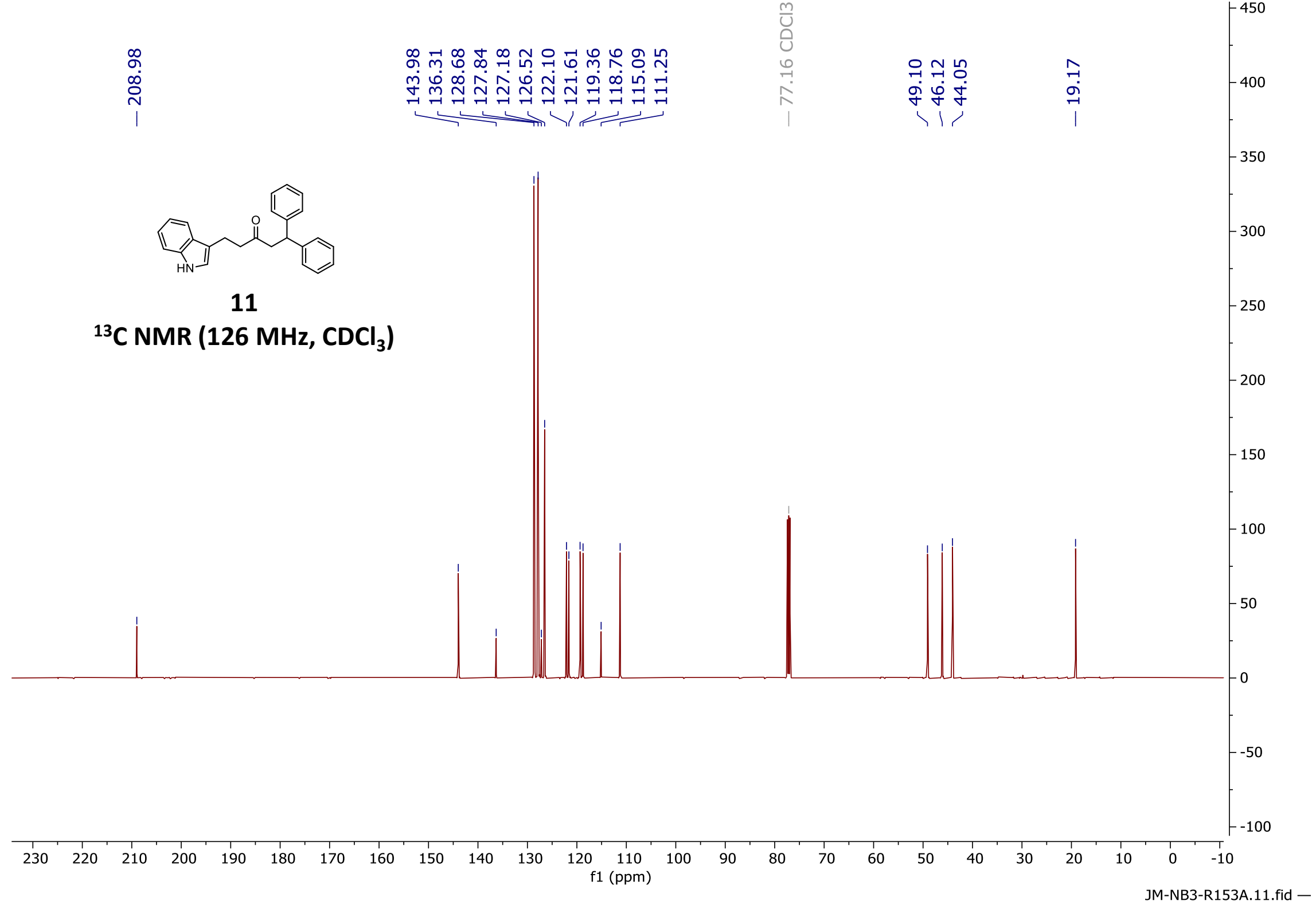




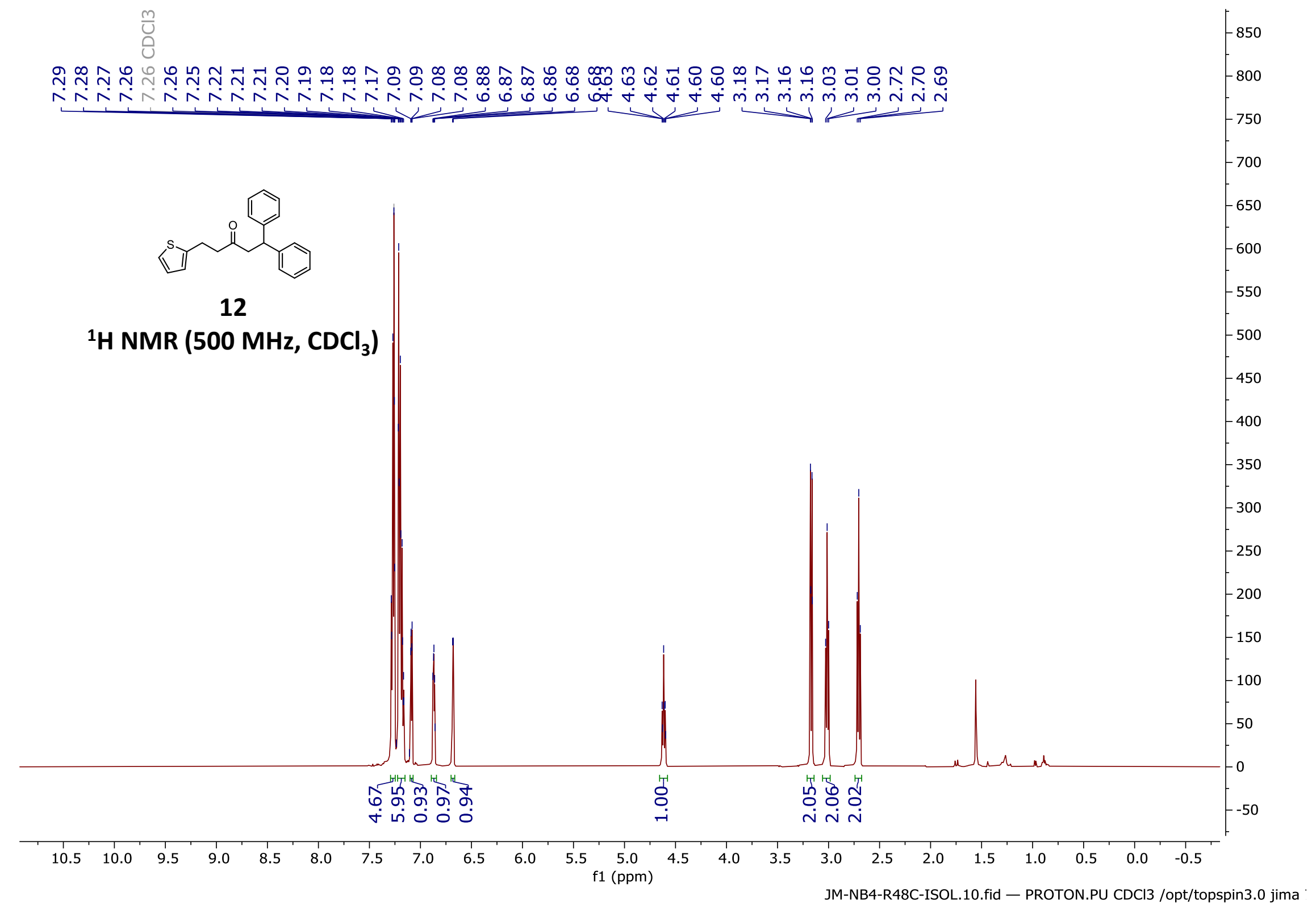




$$
\text { 些 }
$$




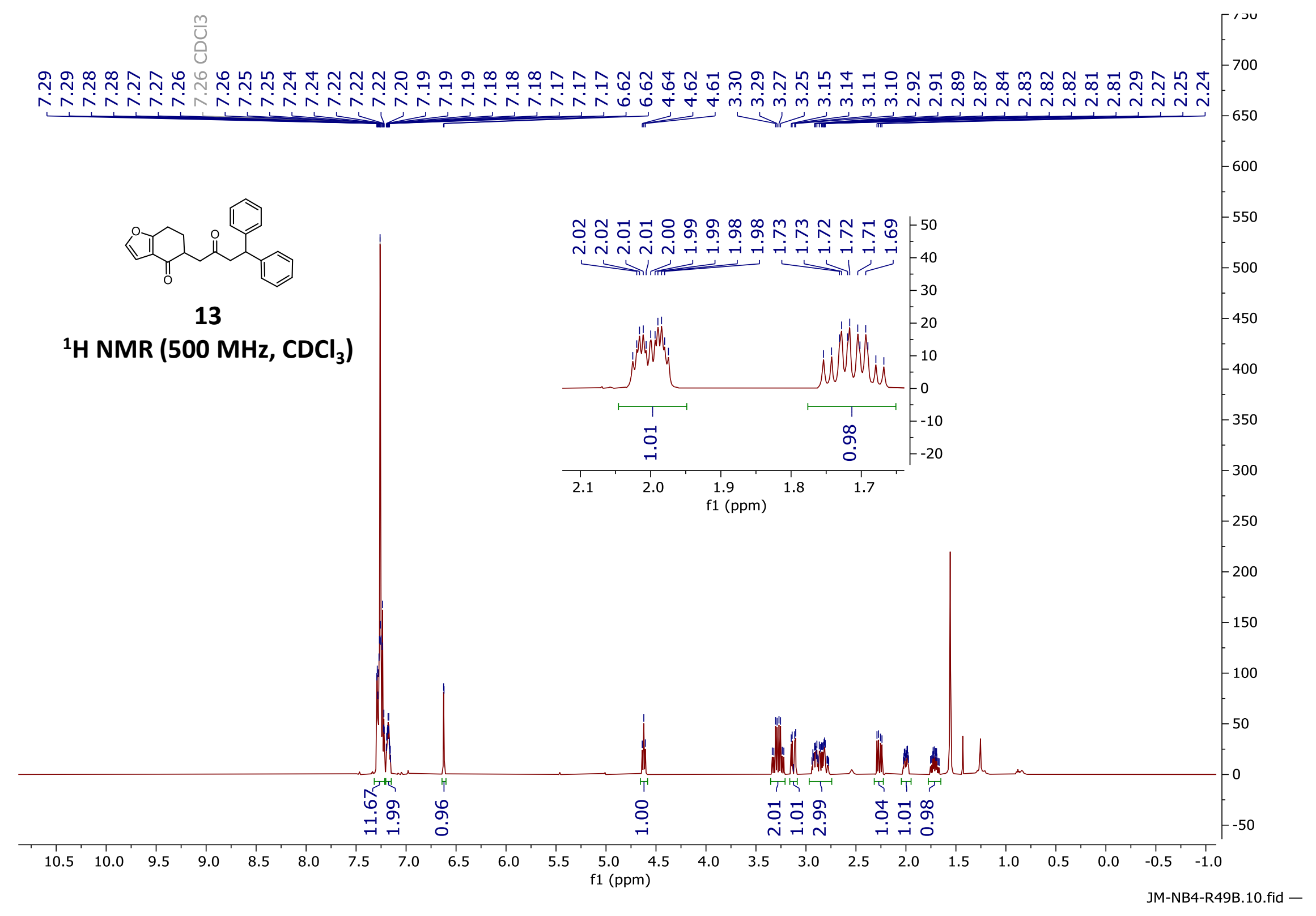




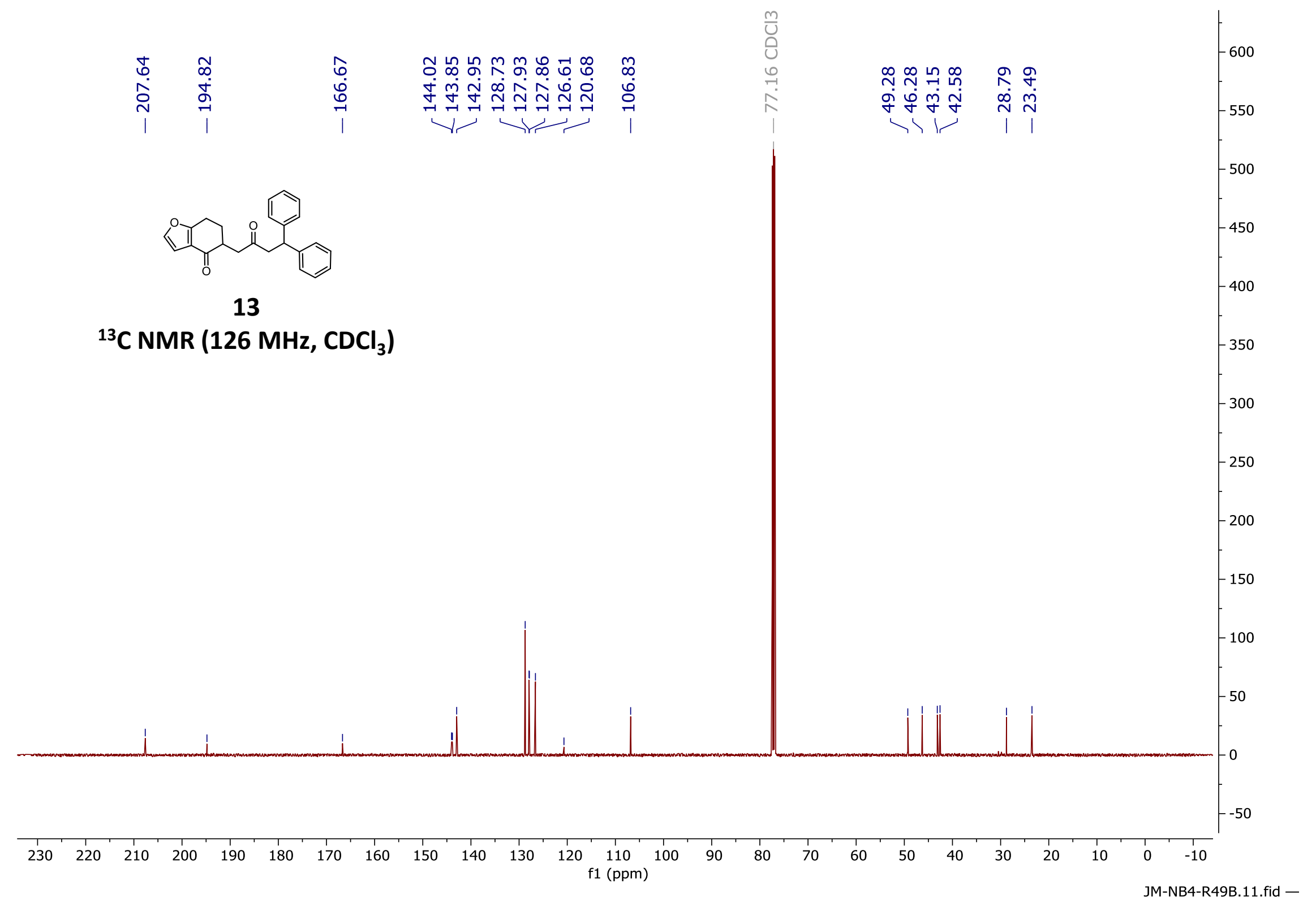




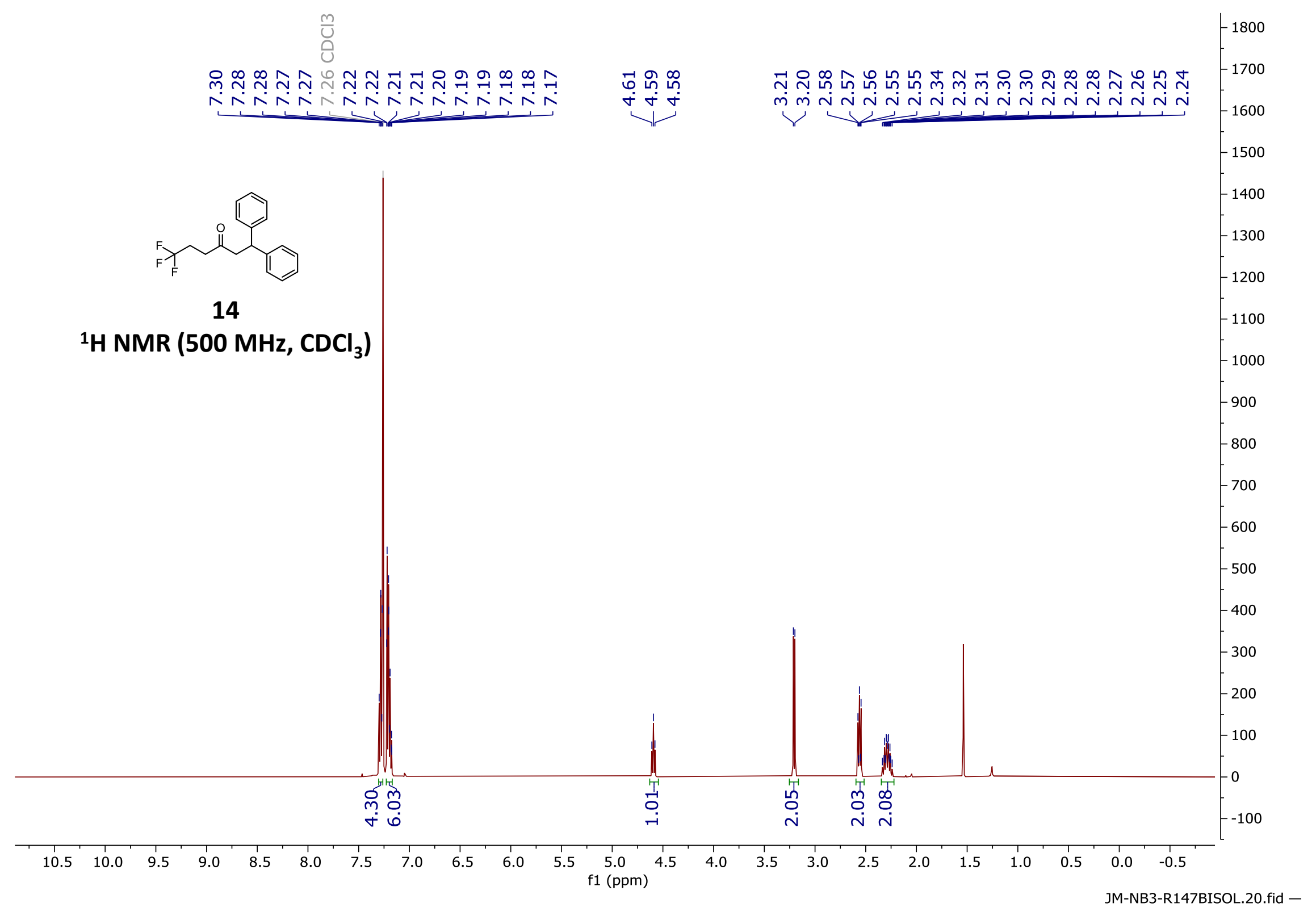




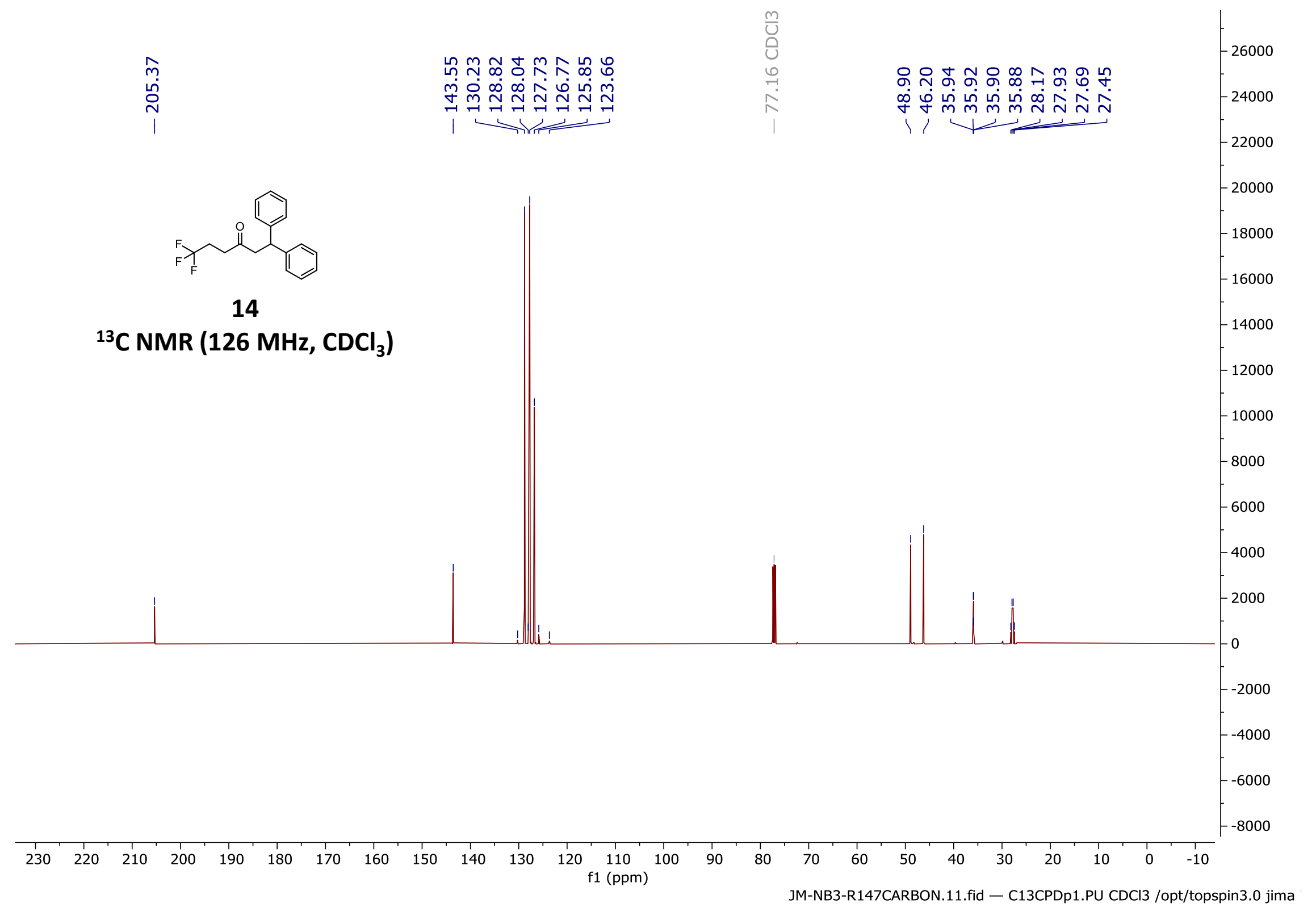




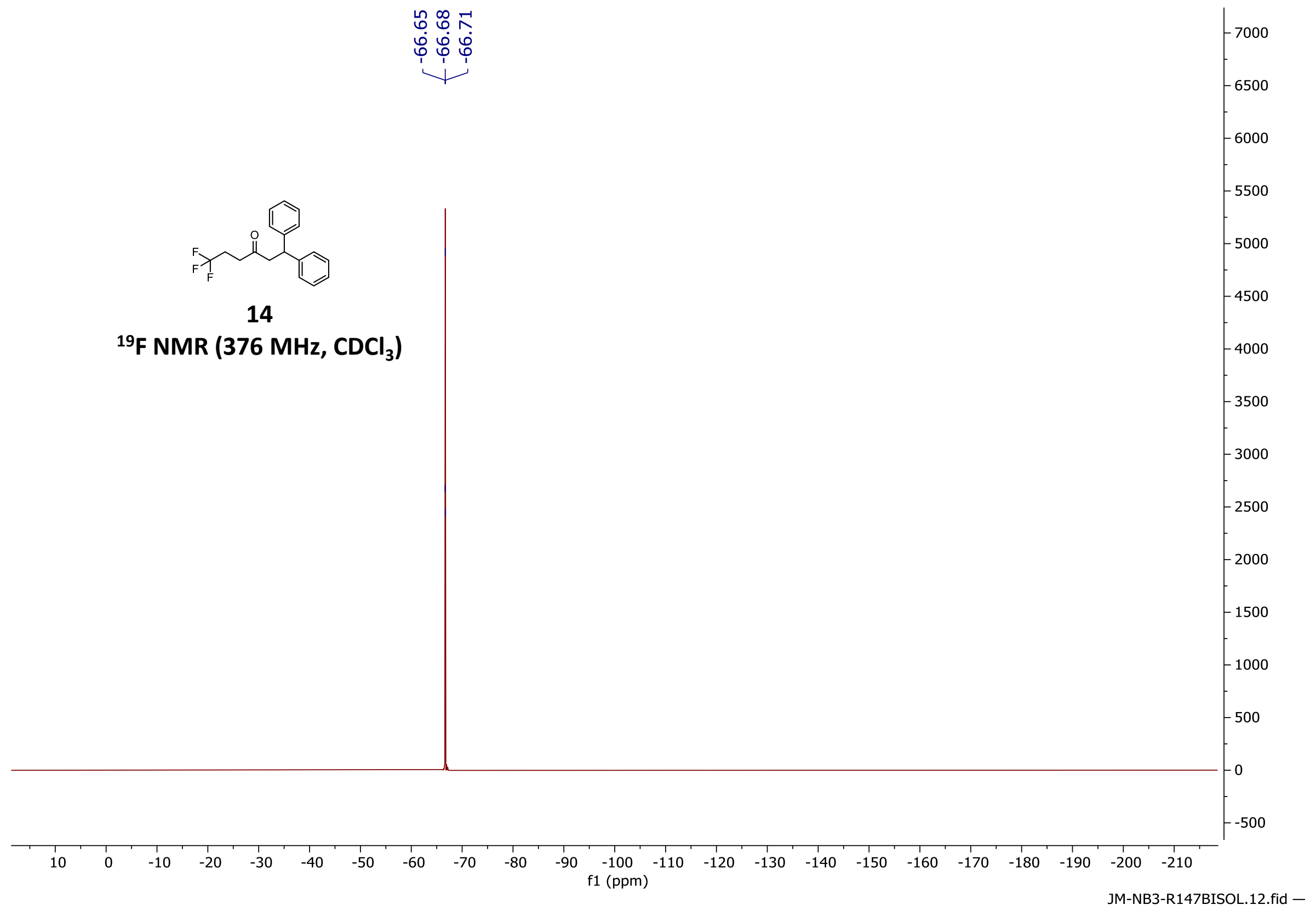




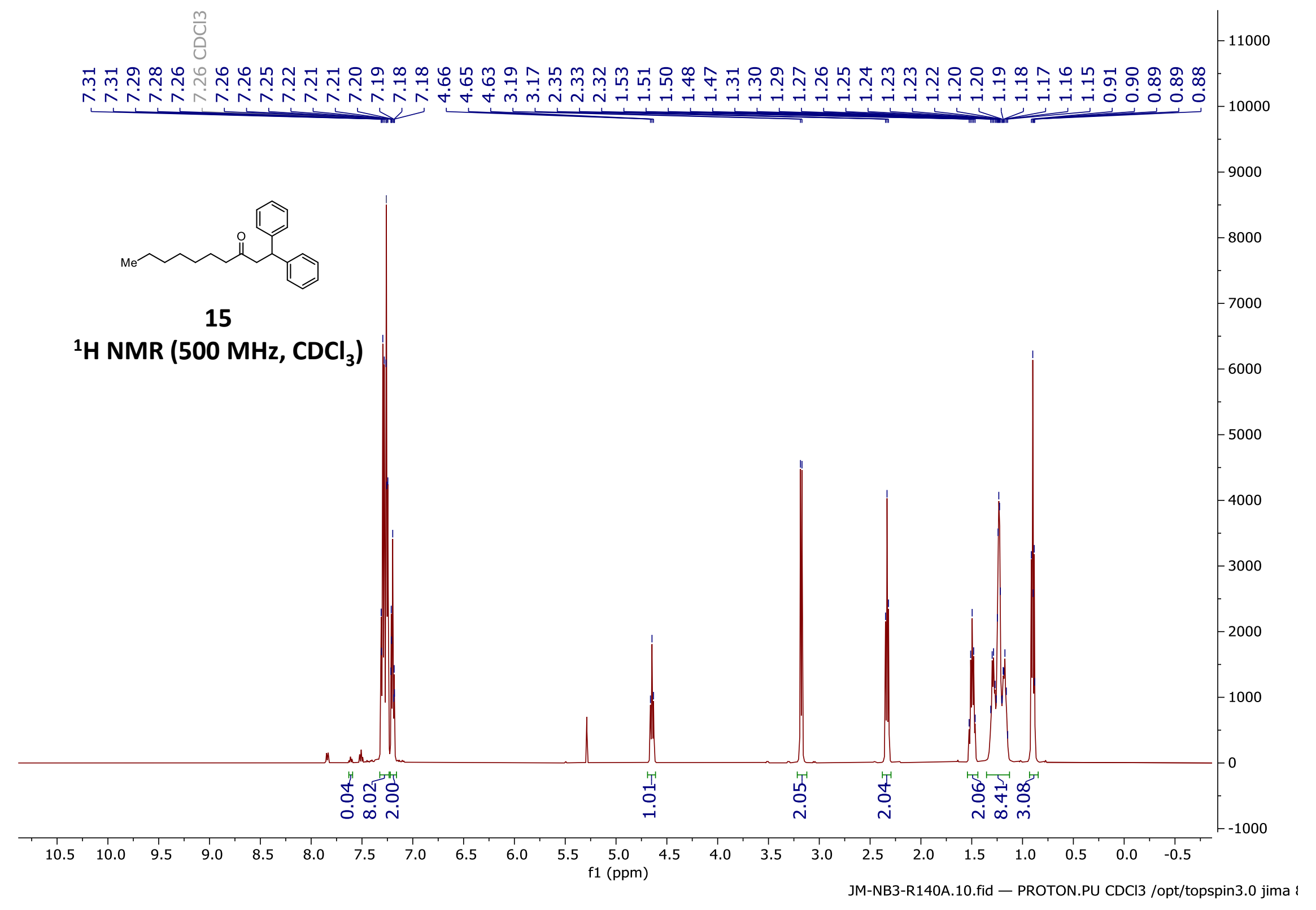




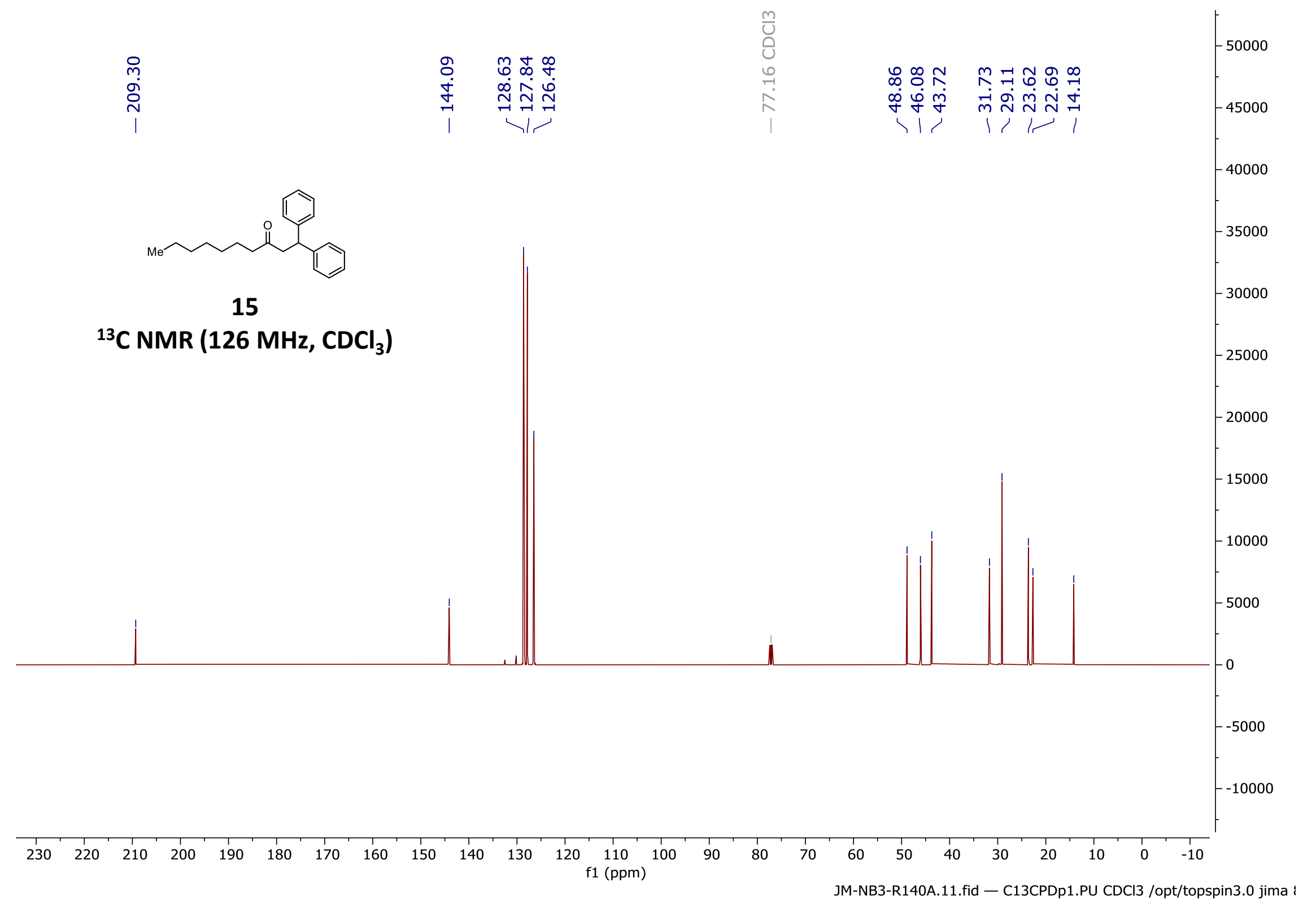




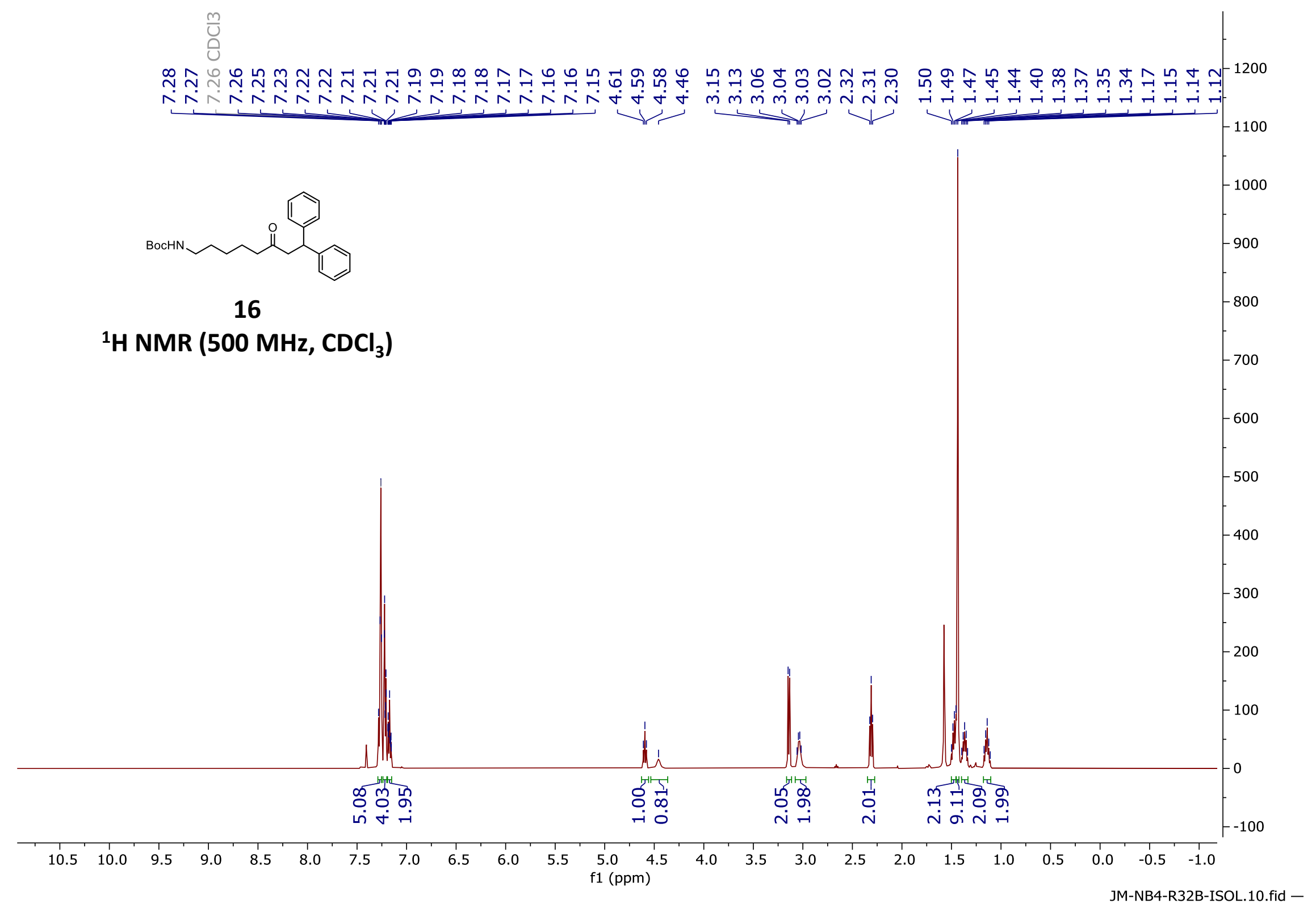




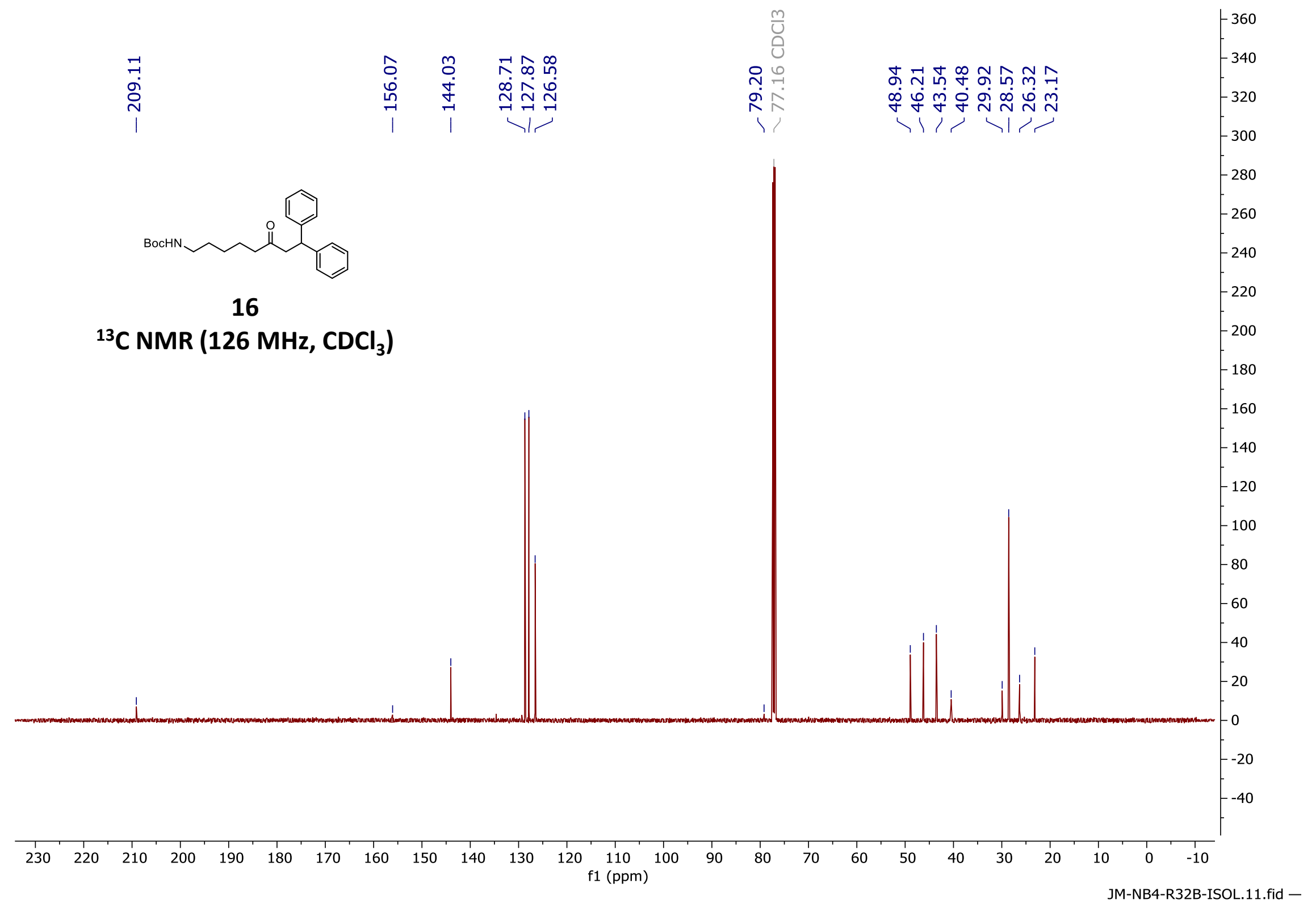




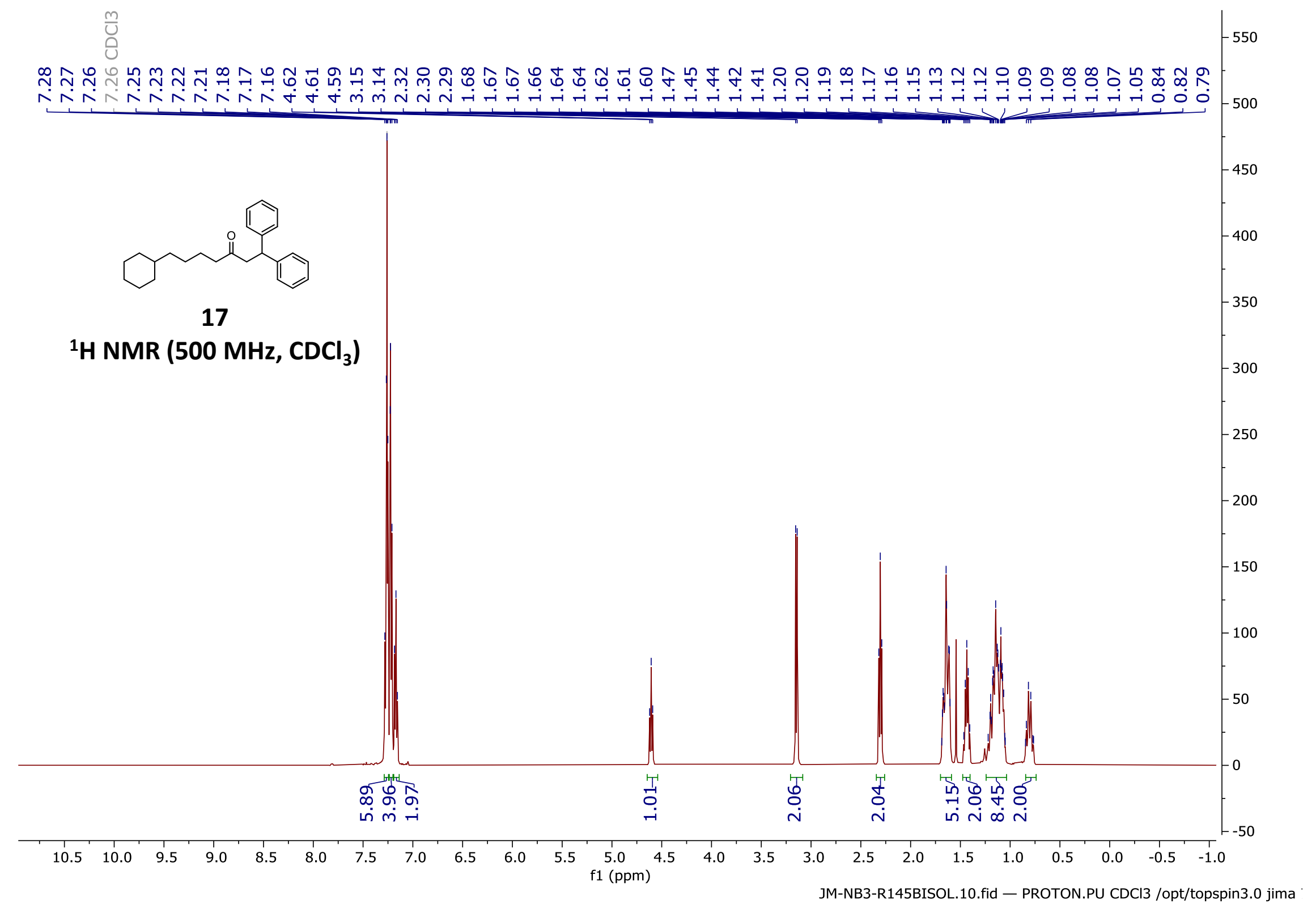




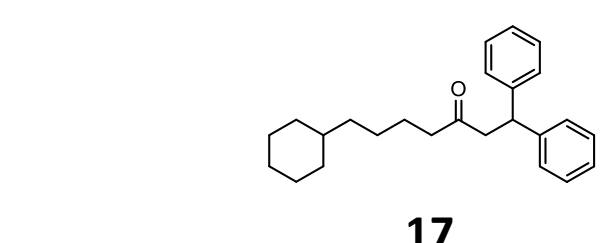

${ }^{13} \mathrm{C} \mathrm{NMR}\left(126 \mathrm{MHz}, \mathrm{CDCl}_{3}\right)$

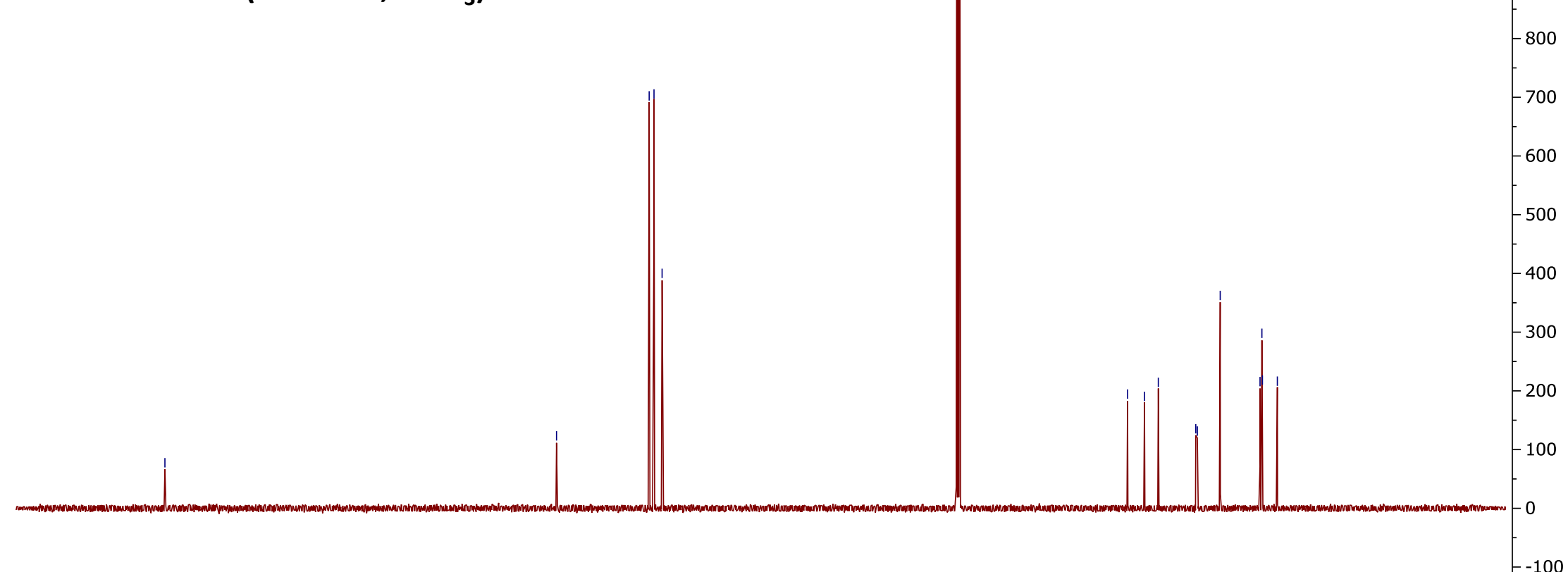




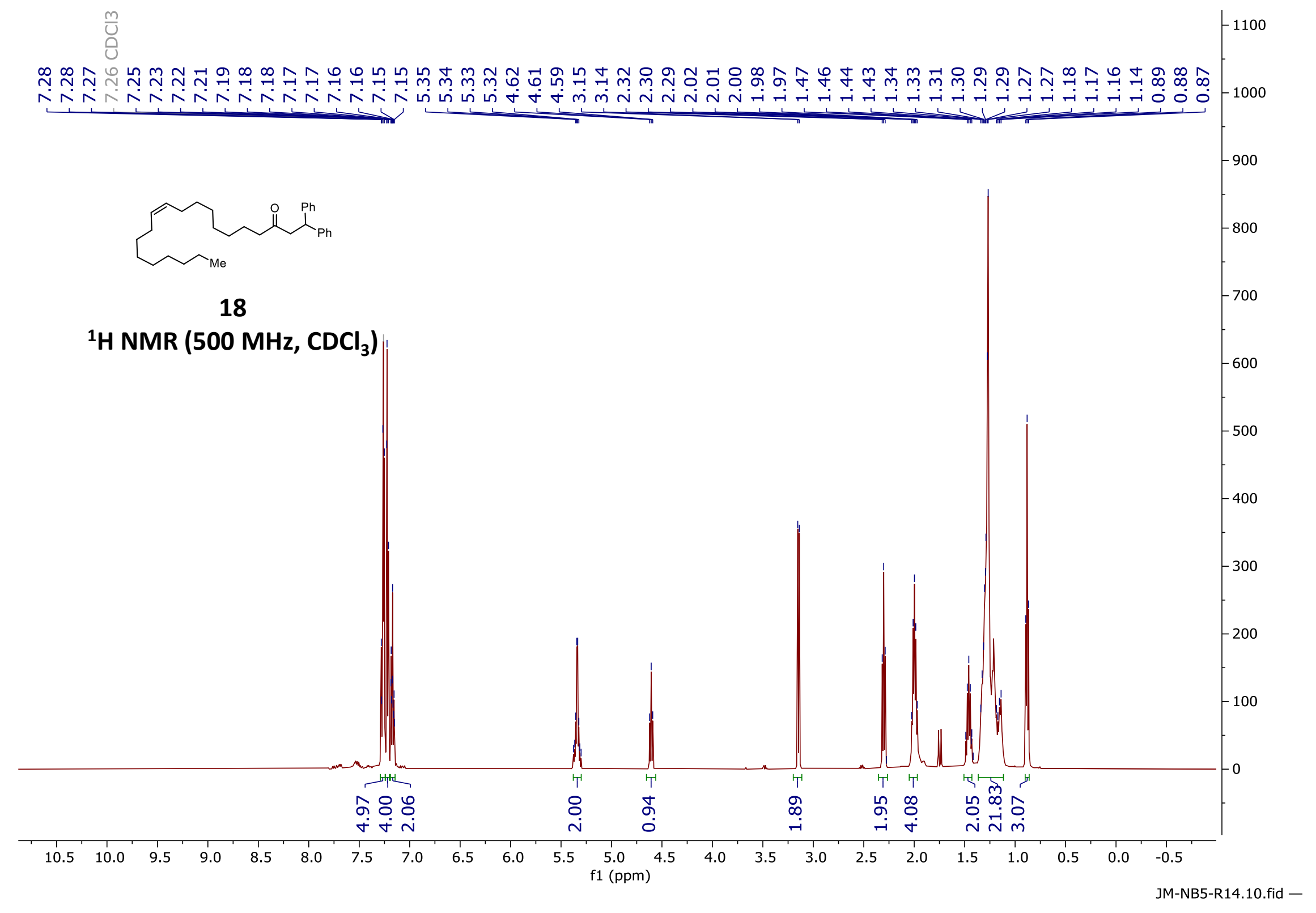




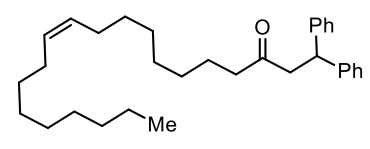

18

${ }^{13} \mathrm{C}$ NMR (126 MHz, $\mathrm{CDCl}_{3}$ )

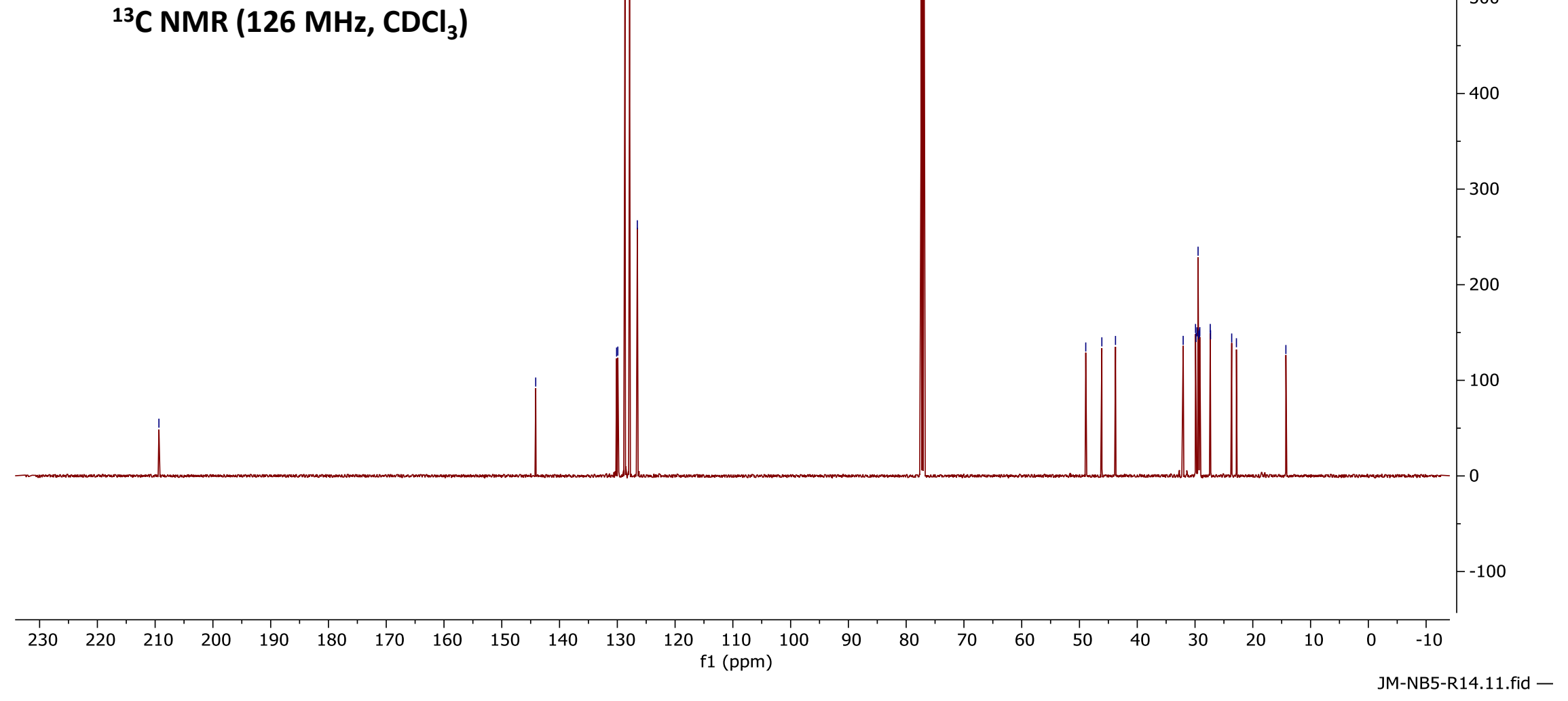




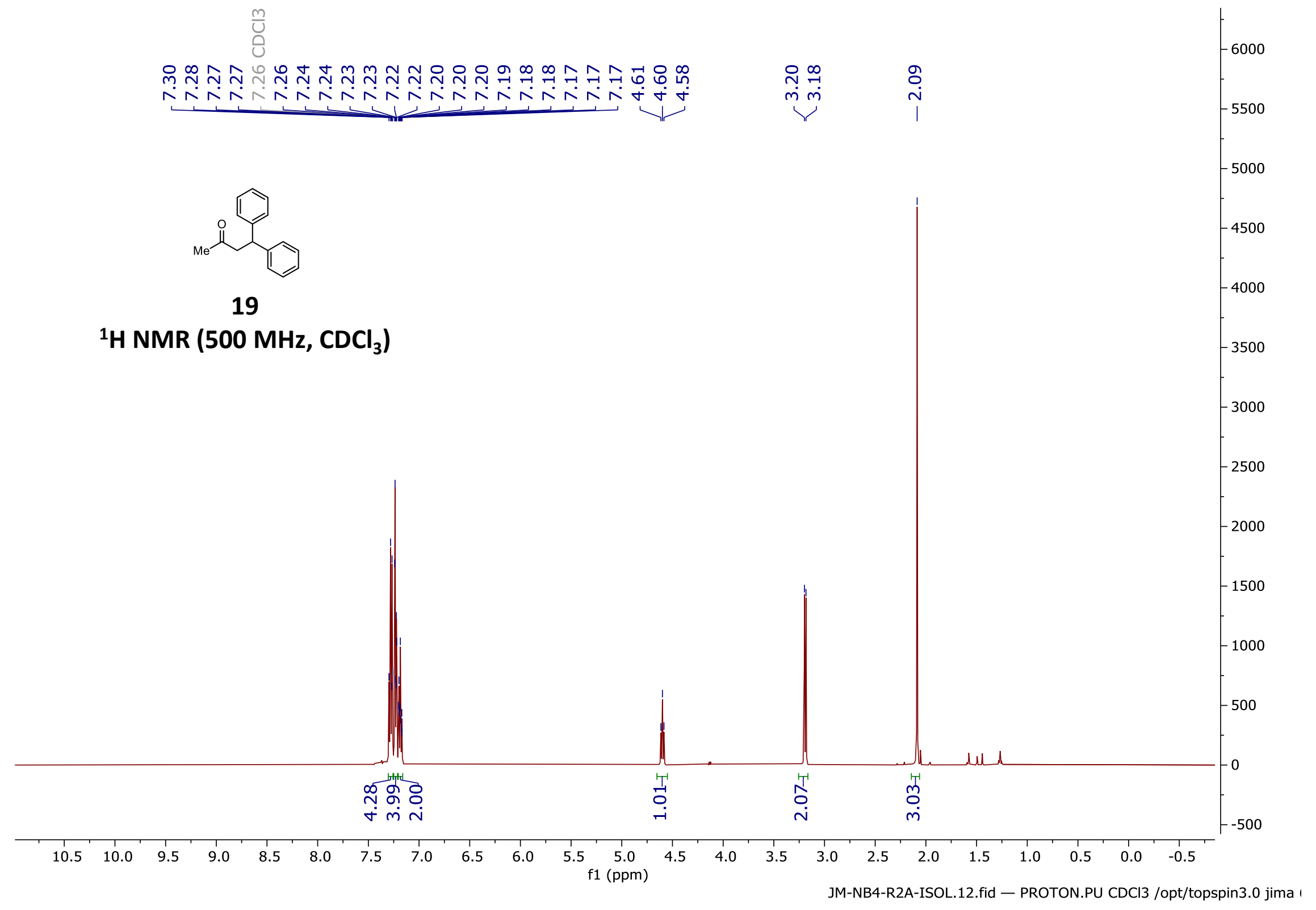




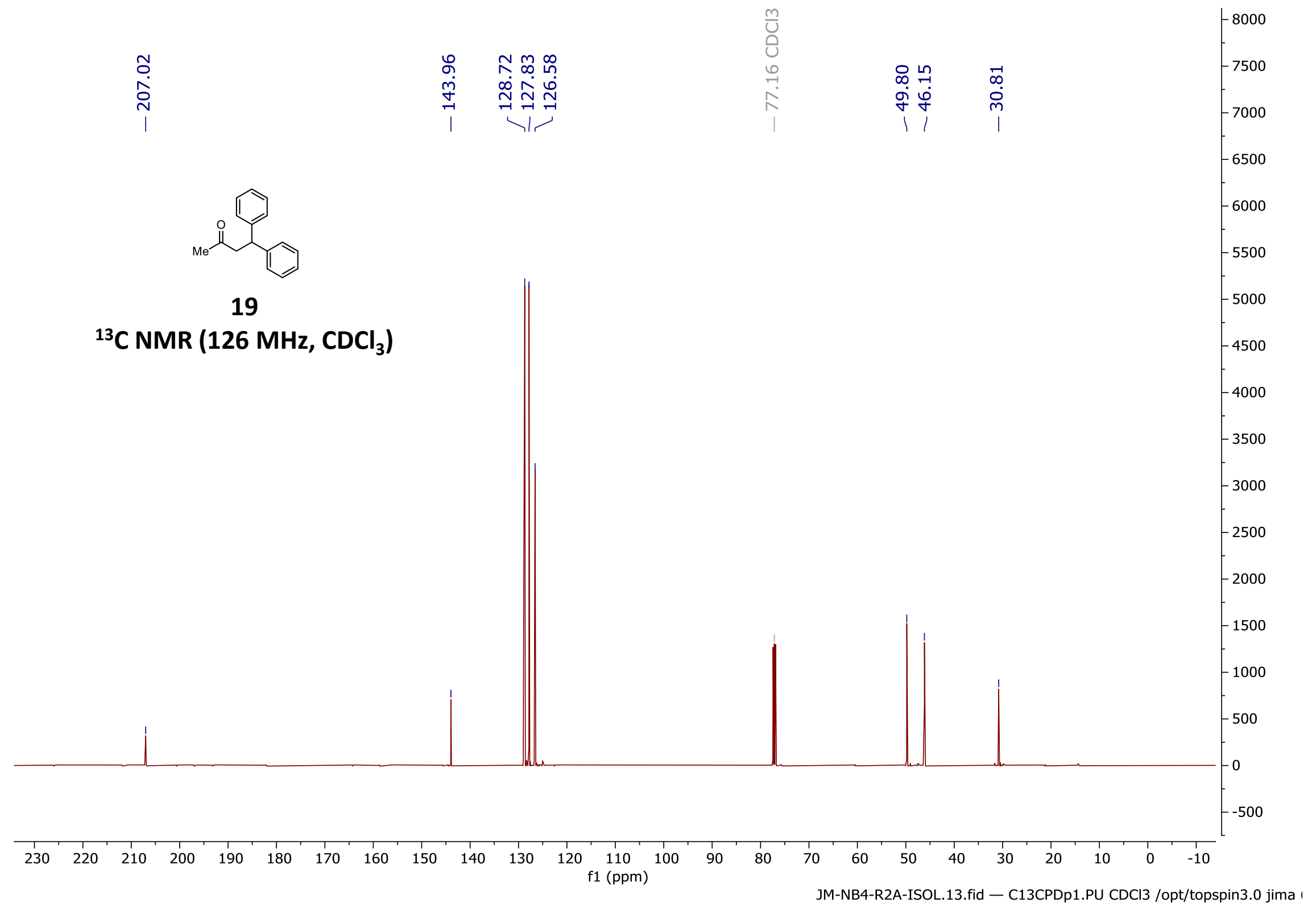


<smiles>CC(C)=CCCC(C)CC(=O)CC(c1ccccc1)c1ccccc1</smiles>

20

${ }^{1} \mathrm{H}$ NMR $\left(500 \mathrm{MHz}, \mathrm{CDCl}_{3}\right)$

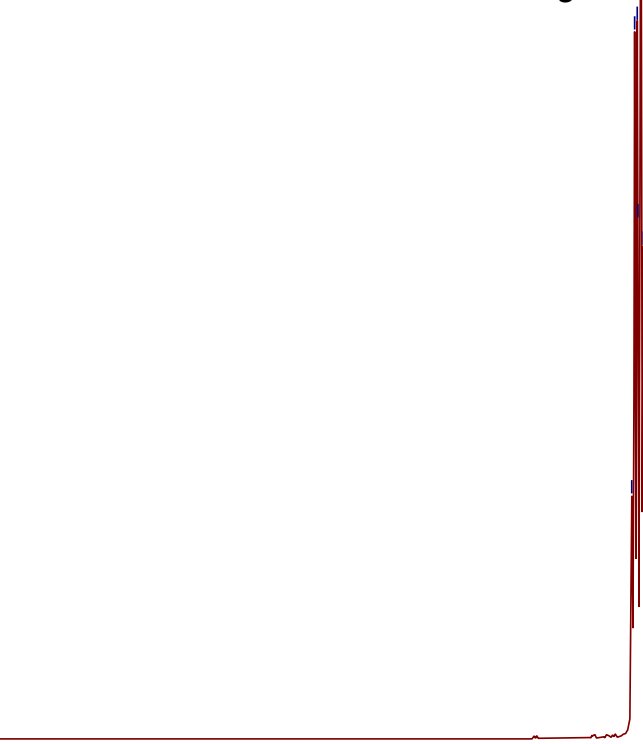

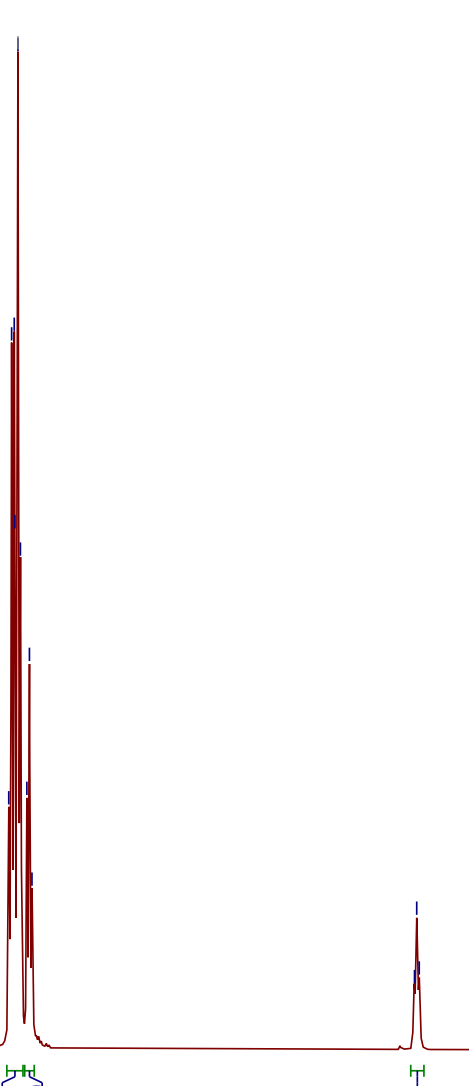

क्ष

咅尊

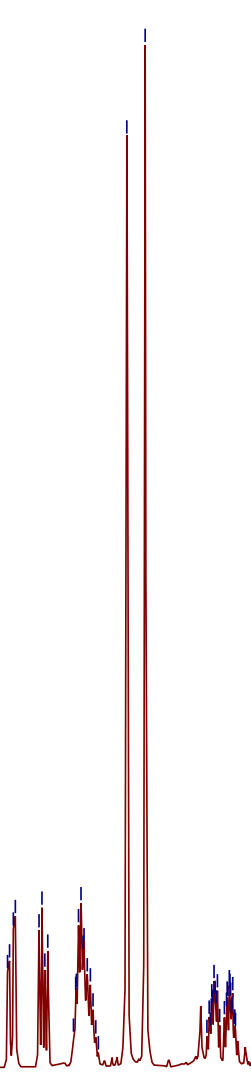

$-5000$

4500

$-4000$

$-3500$

$-3000$

$-2500$

$-2000$

$-1500$

$-1000$ 


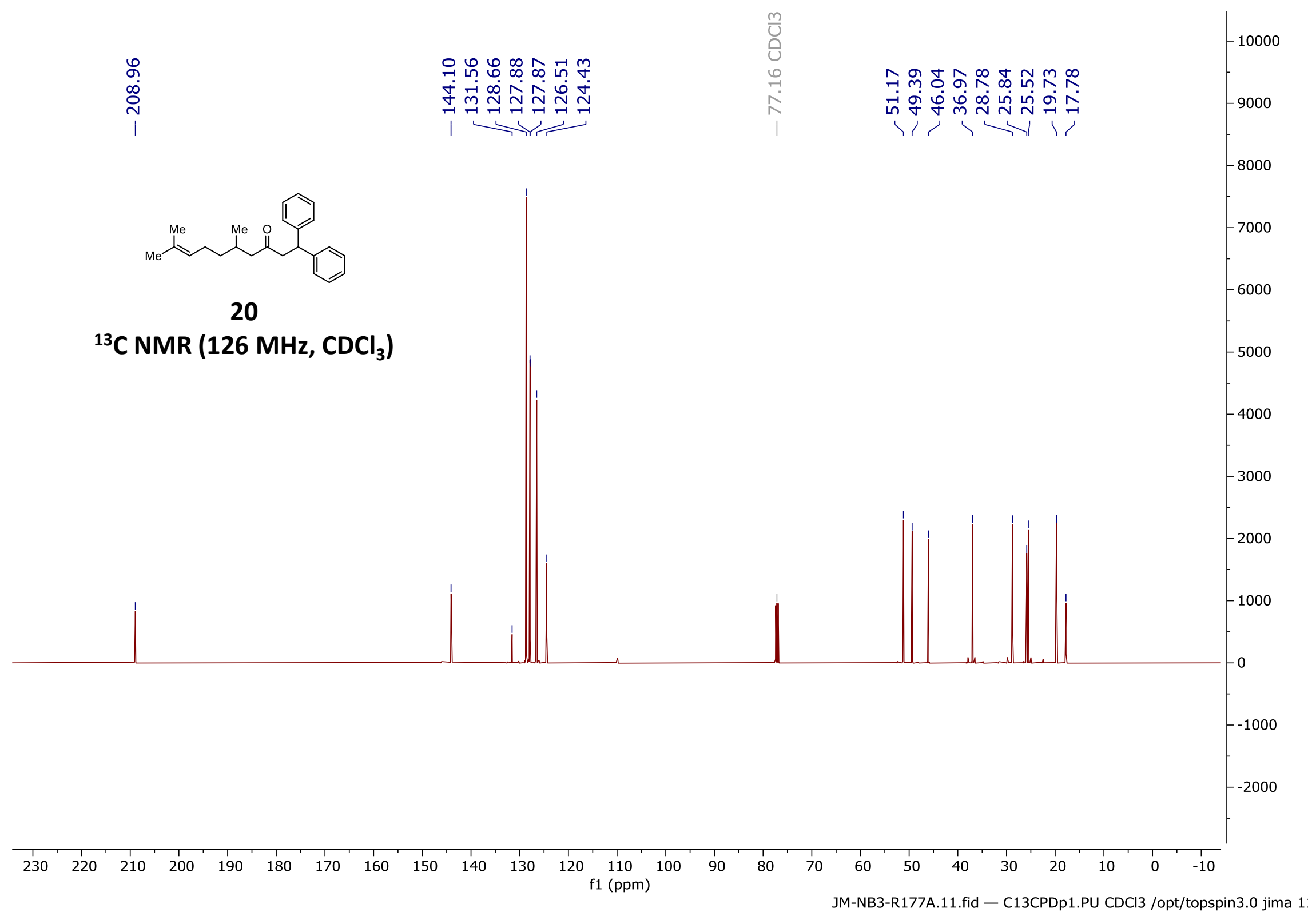




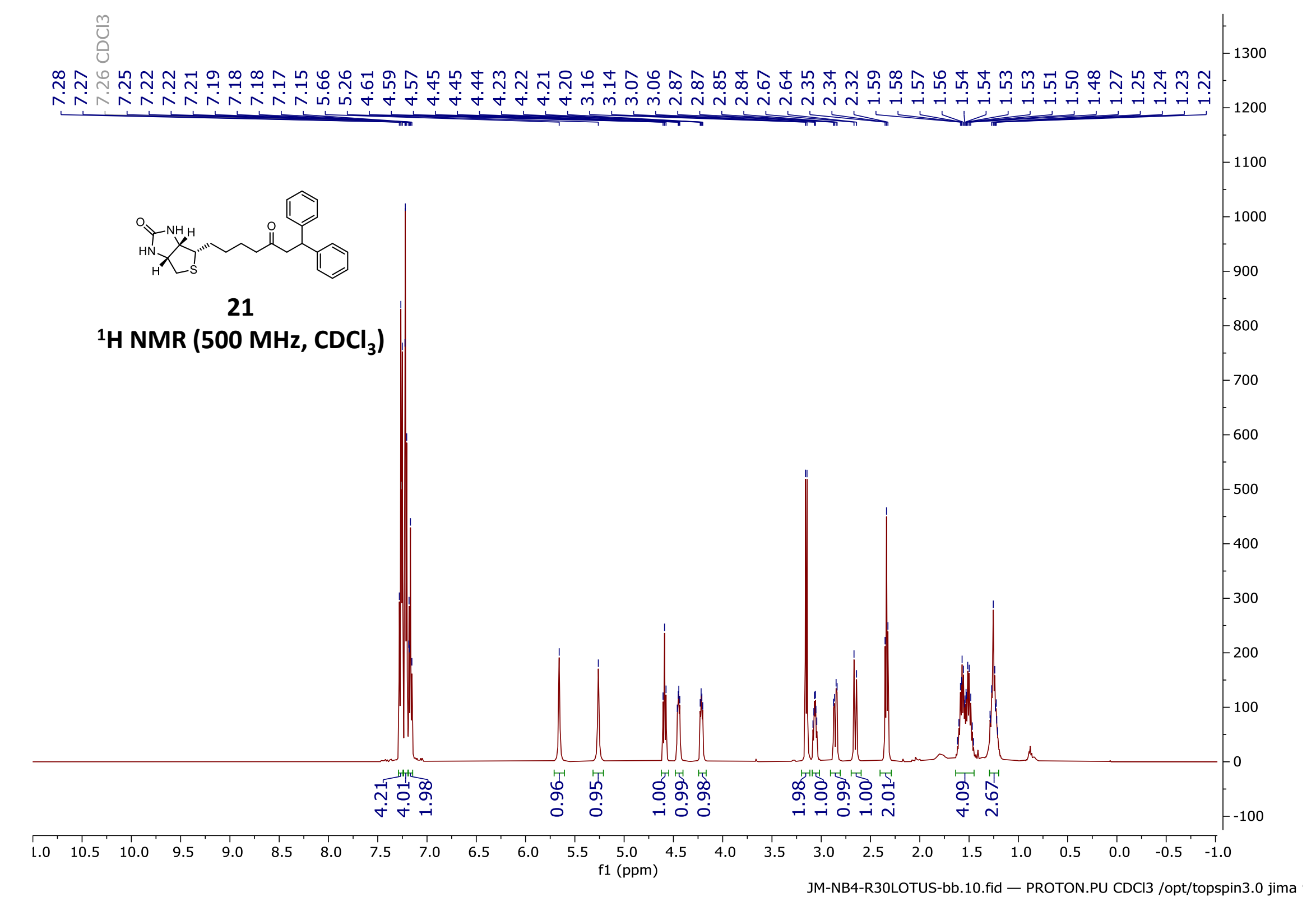




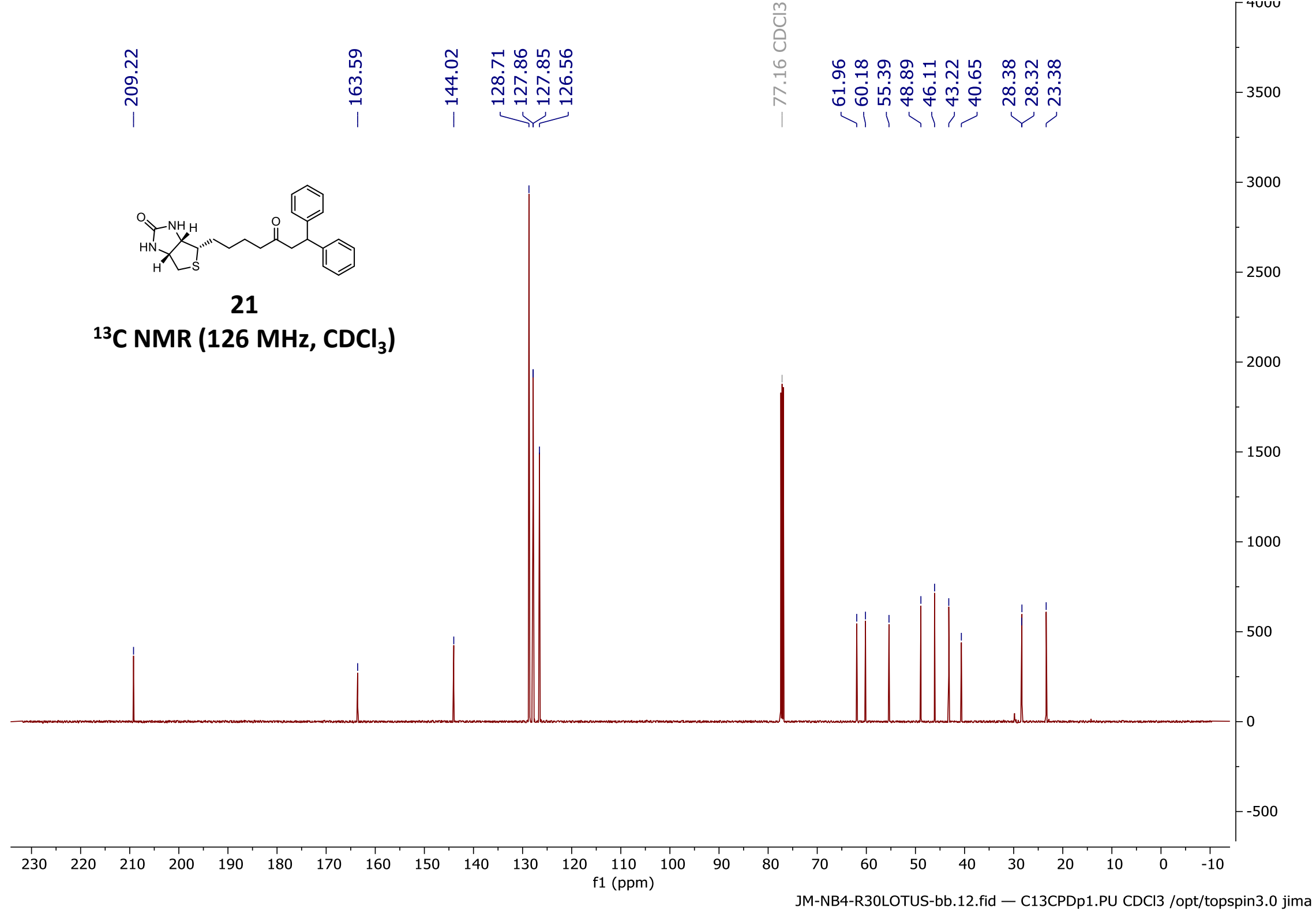




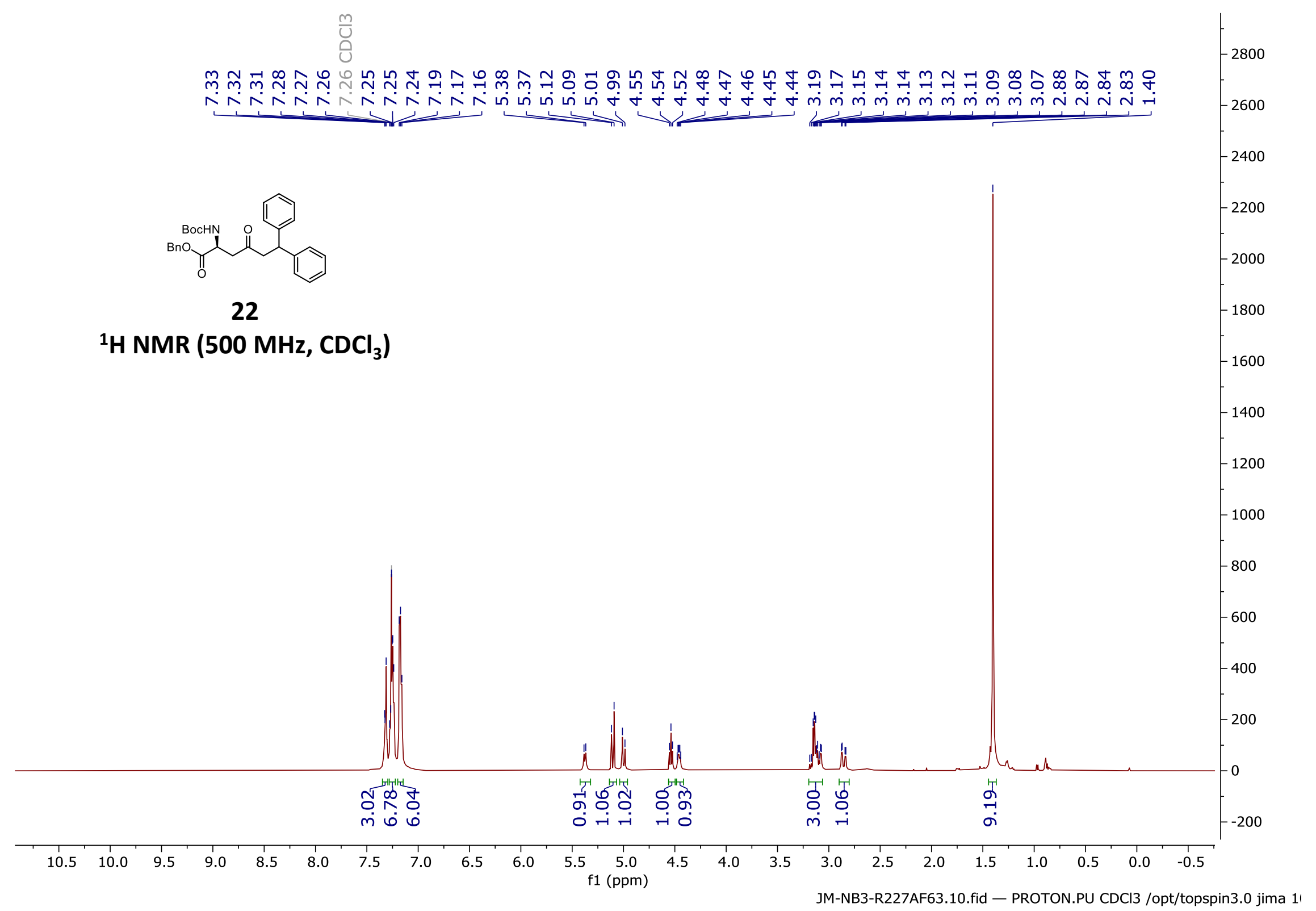




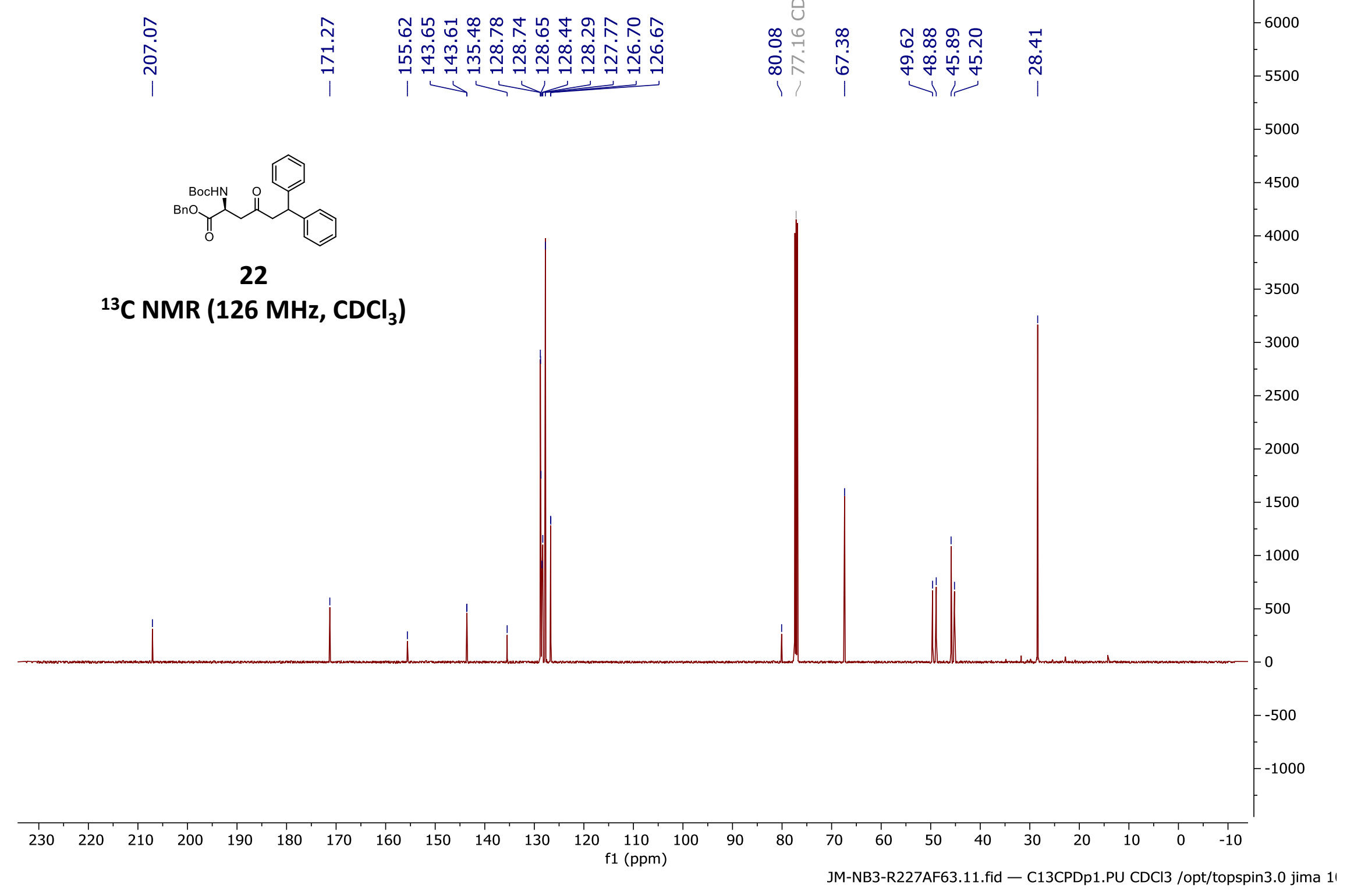




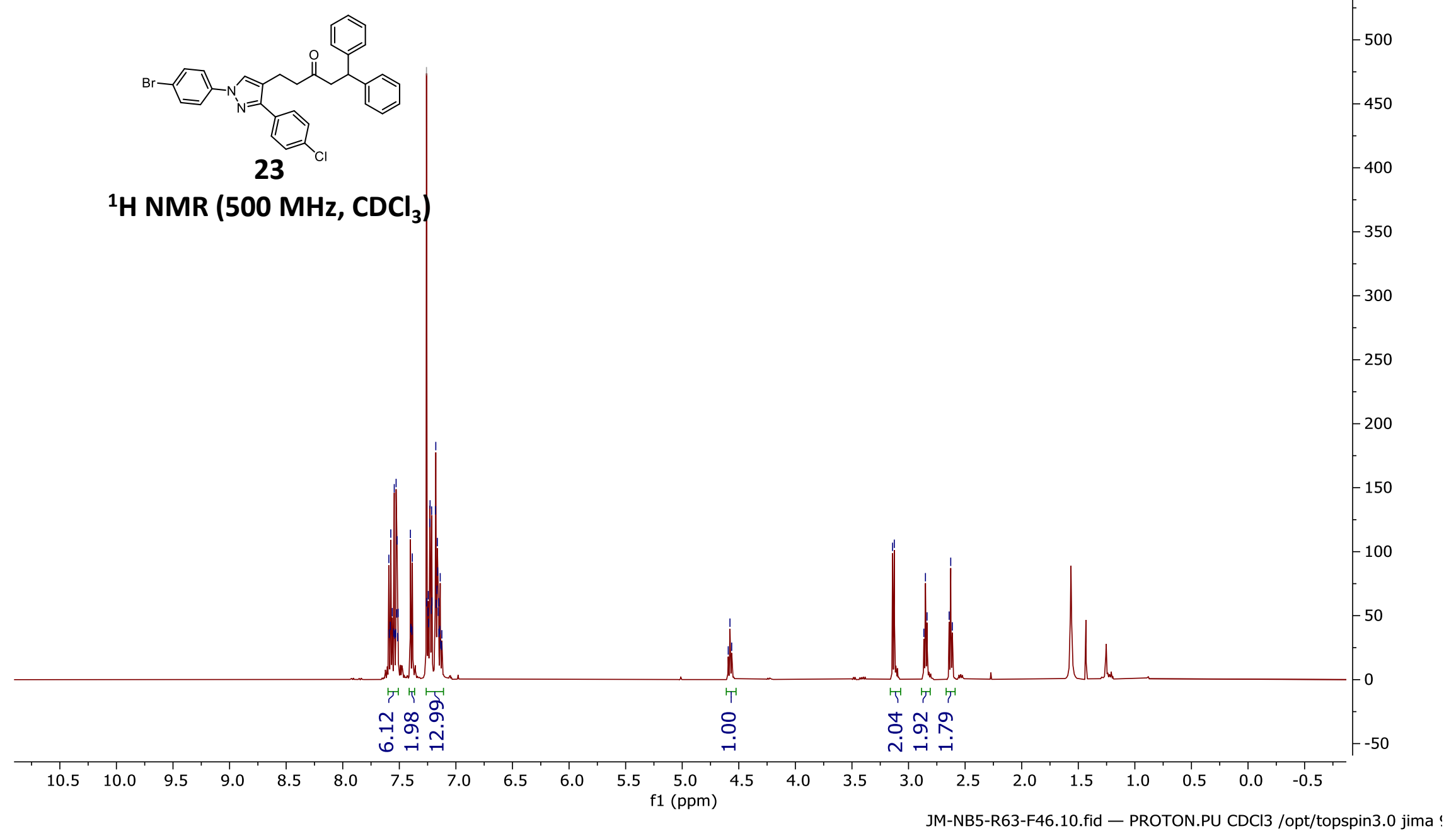




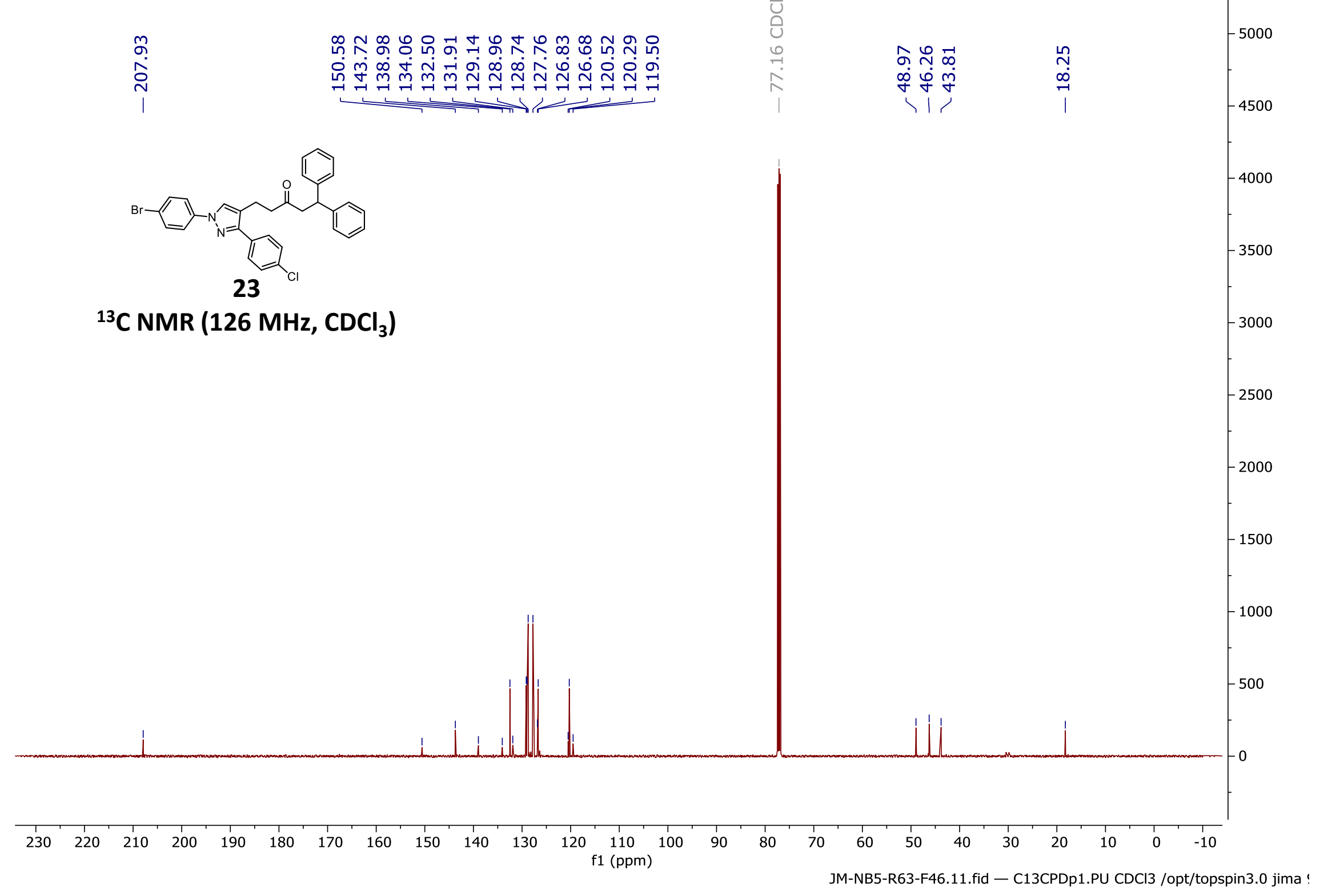


蓆

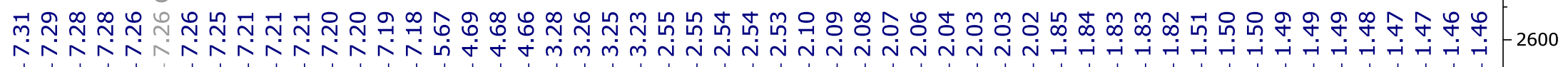

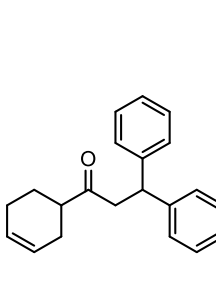

24

${ }^{1} \mathrm{H} \mathrm{NMR}\left(500 \mathrm{MHz}, \mathrm{CDCl}_{3}\right)$

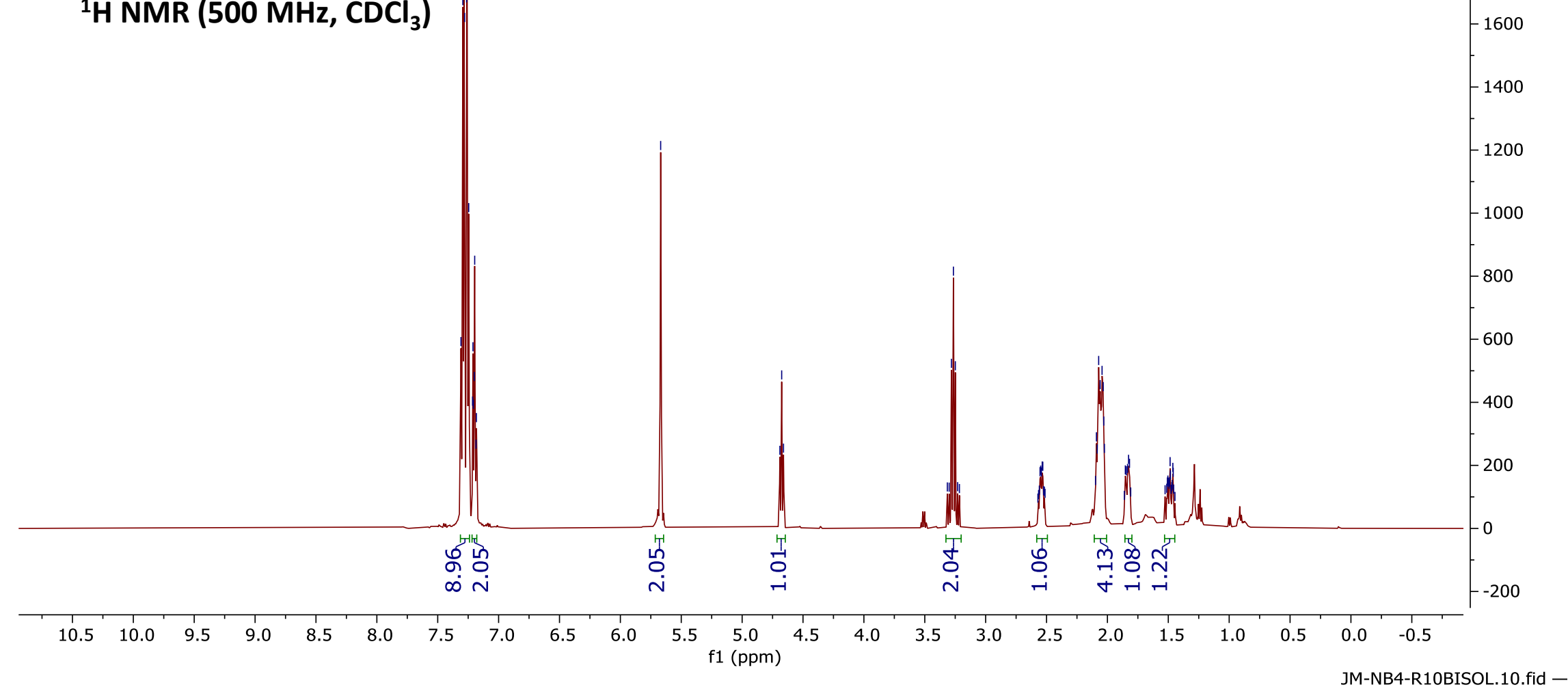




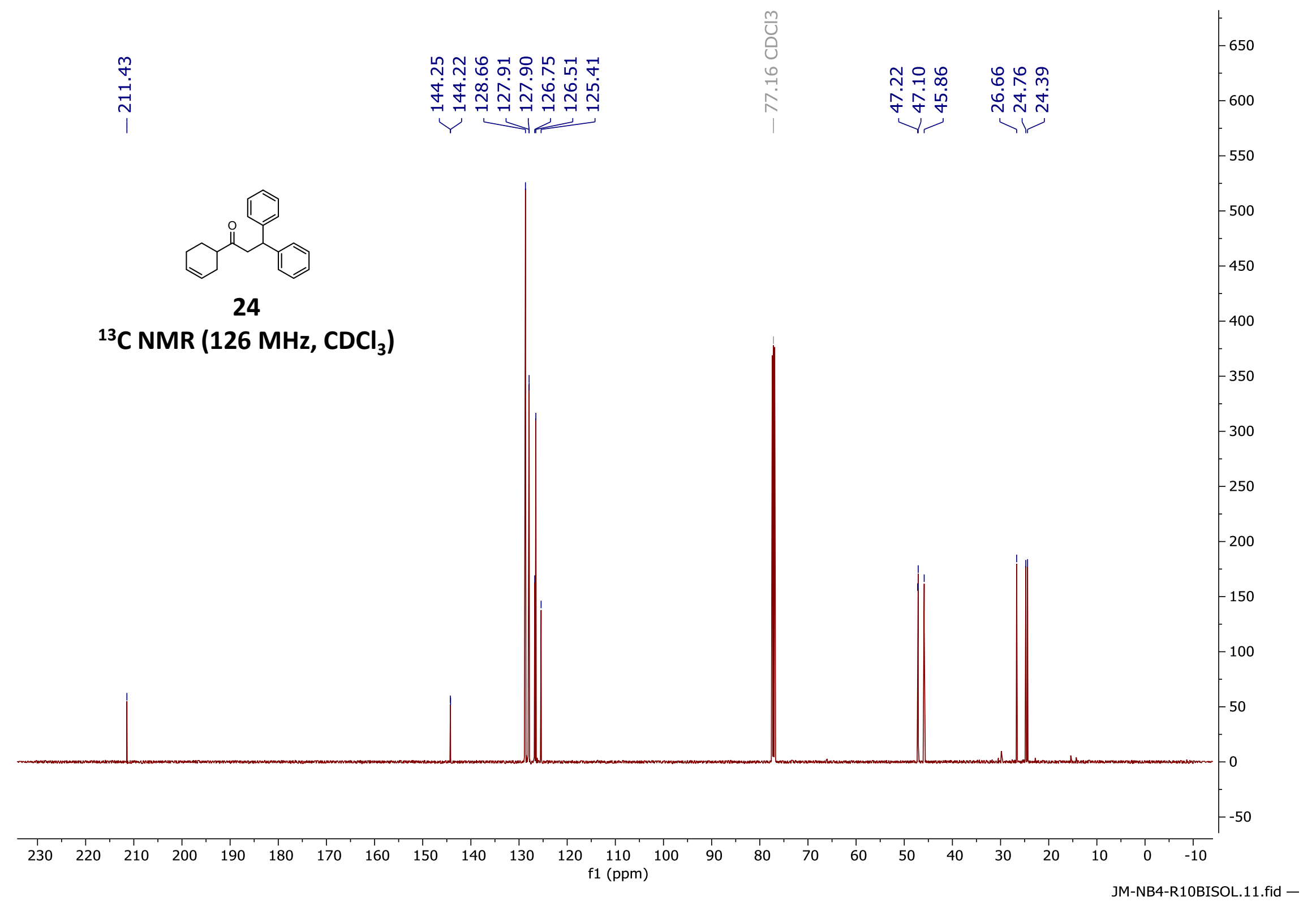




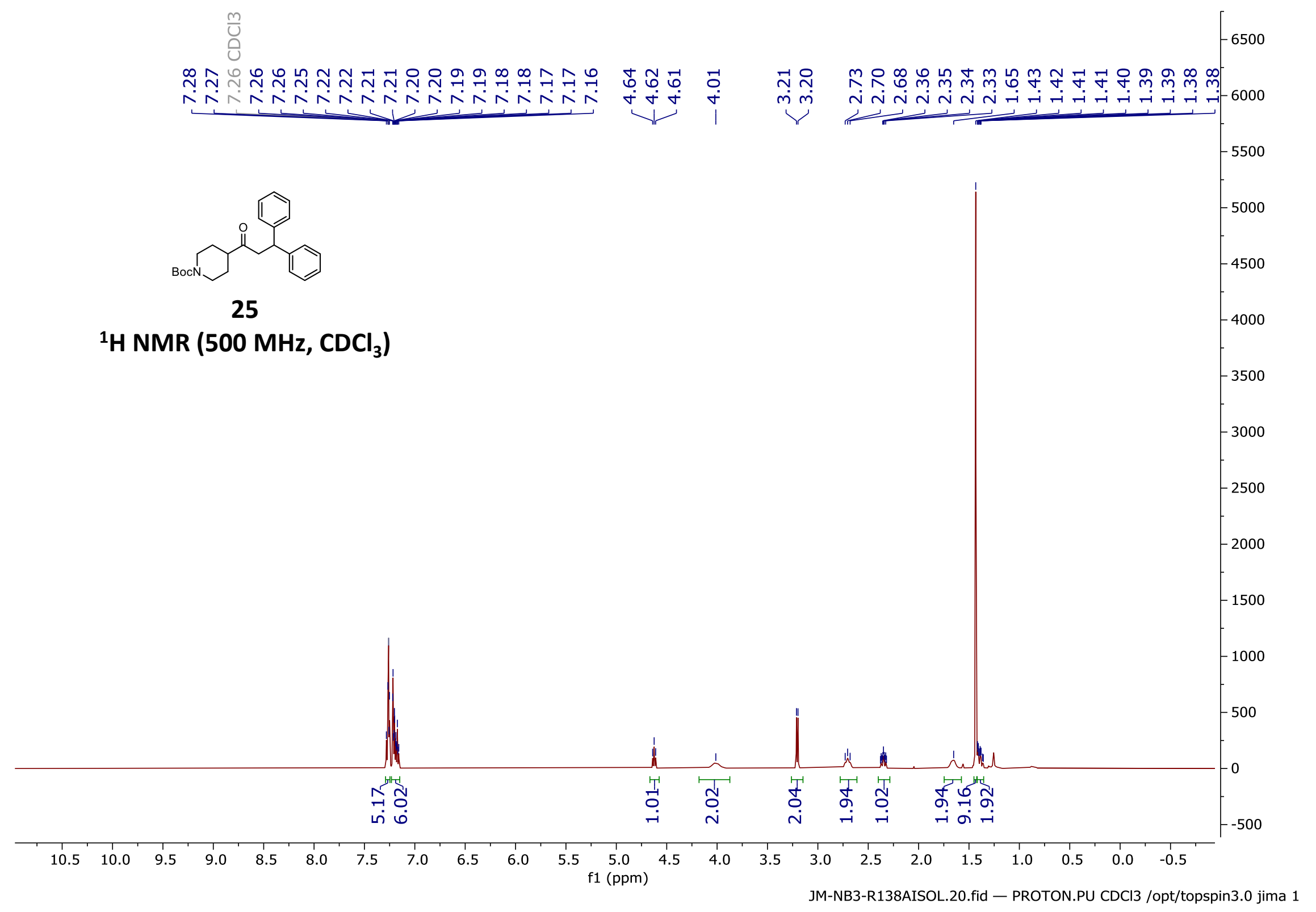




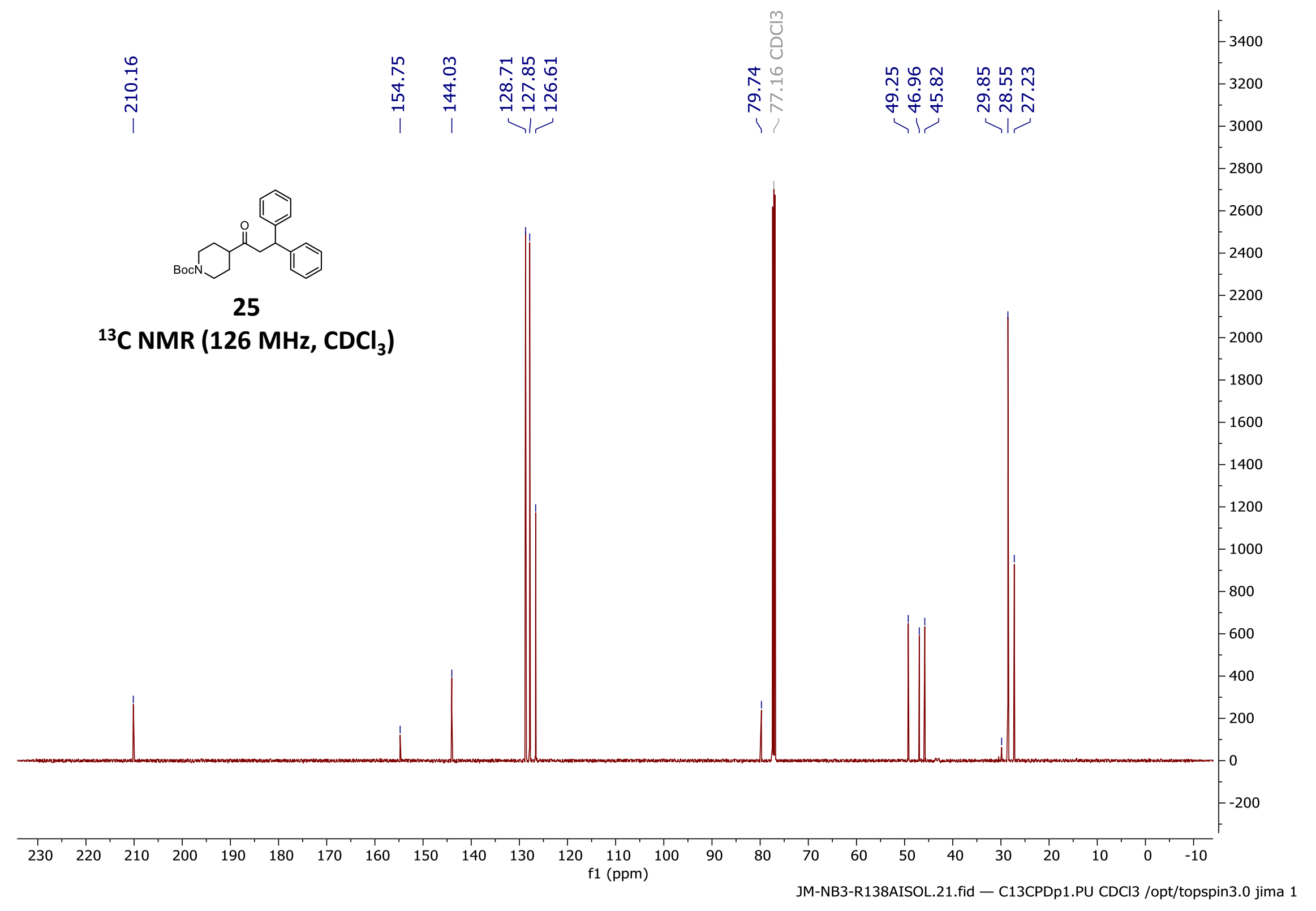




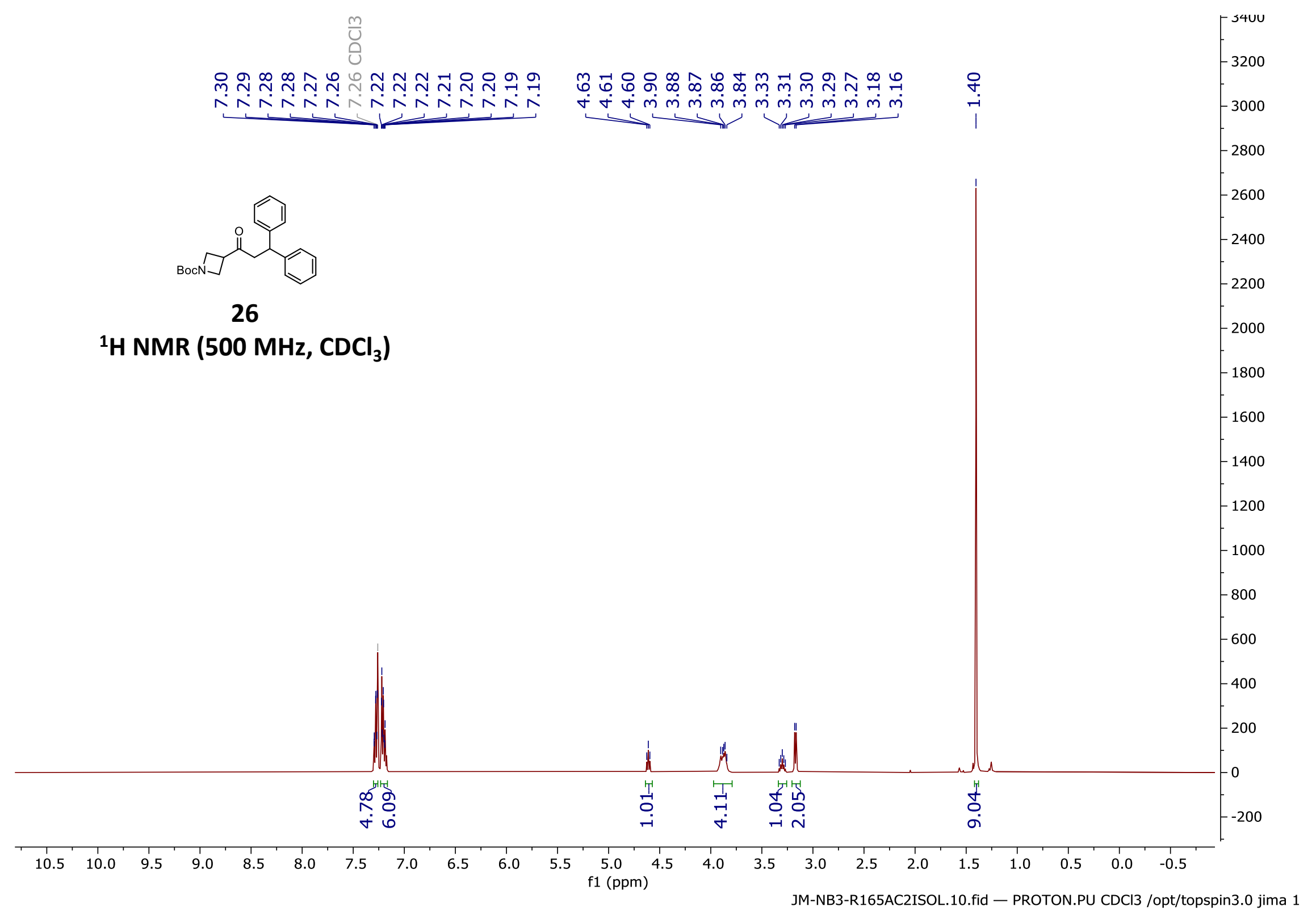




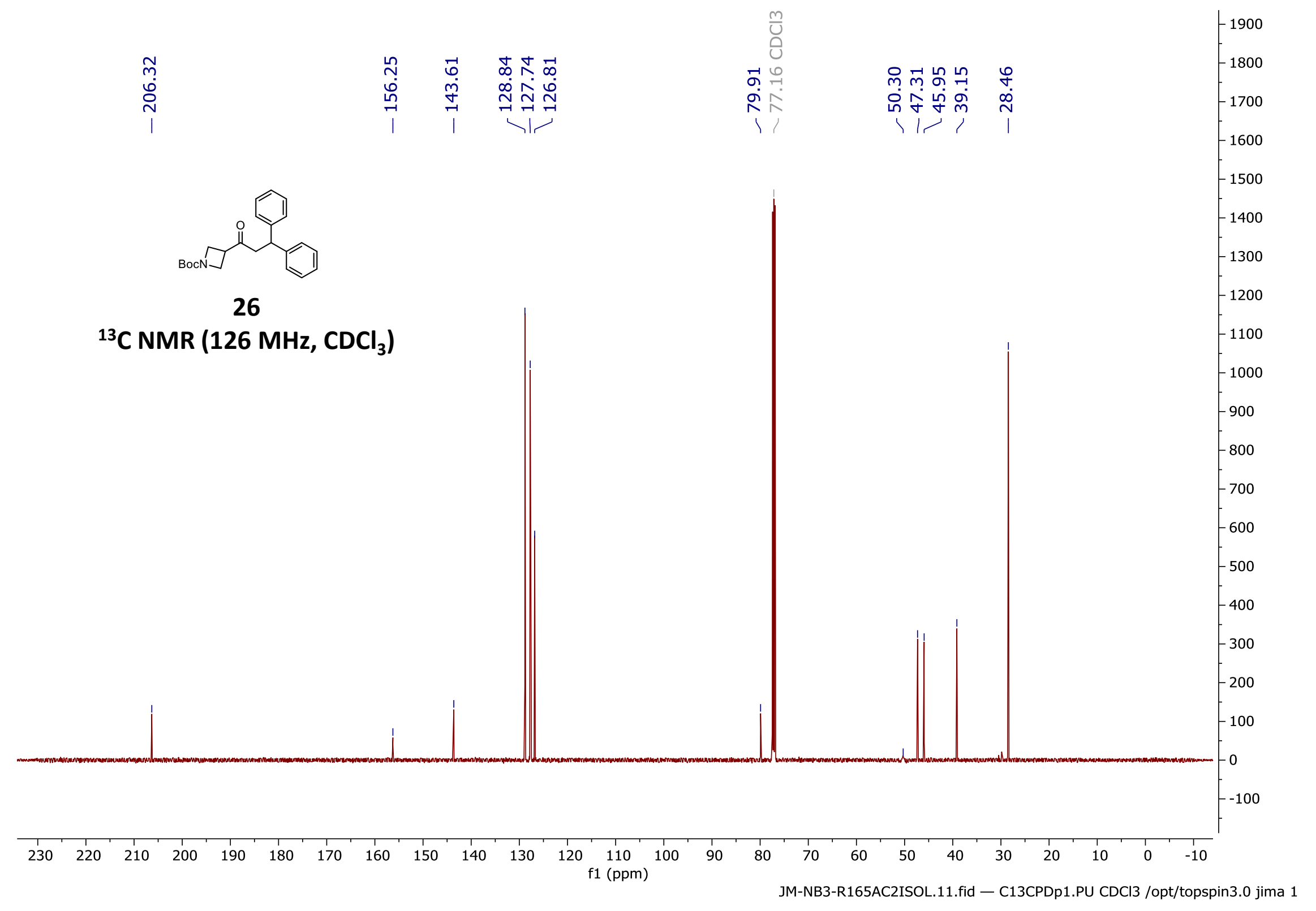




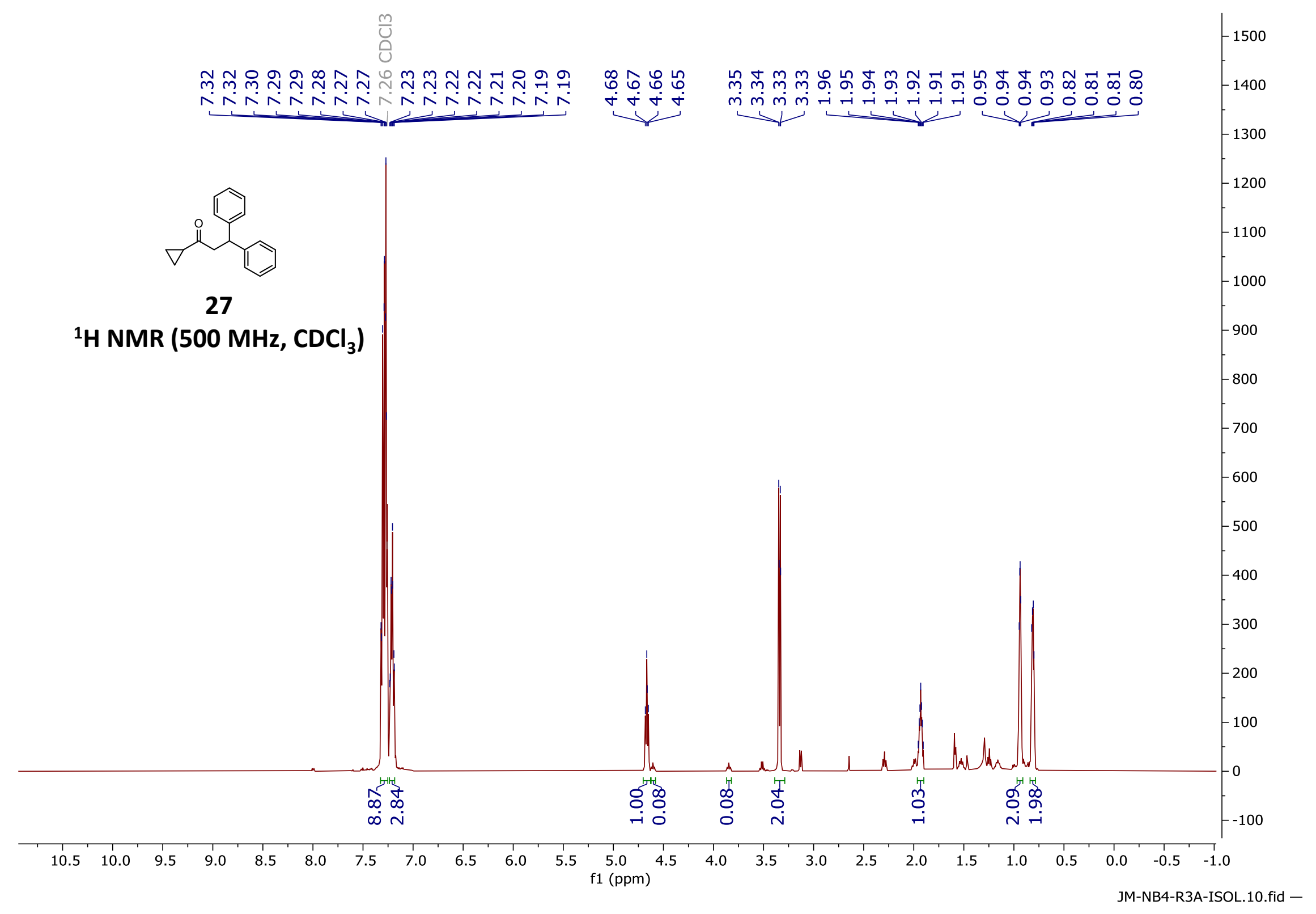


হे
ᄋ
।

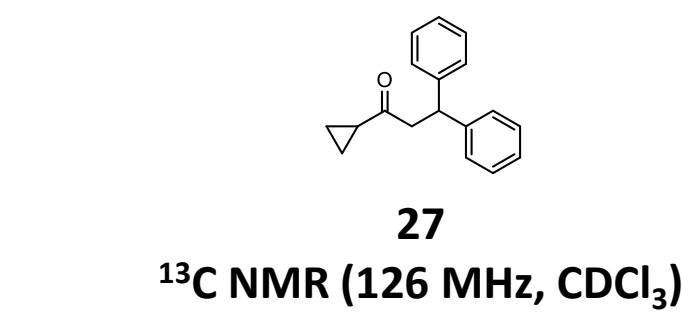

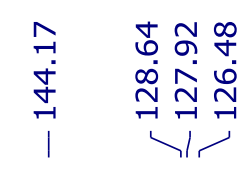

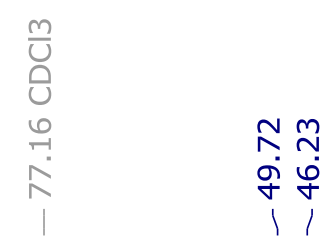

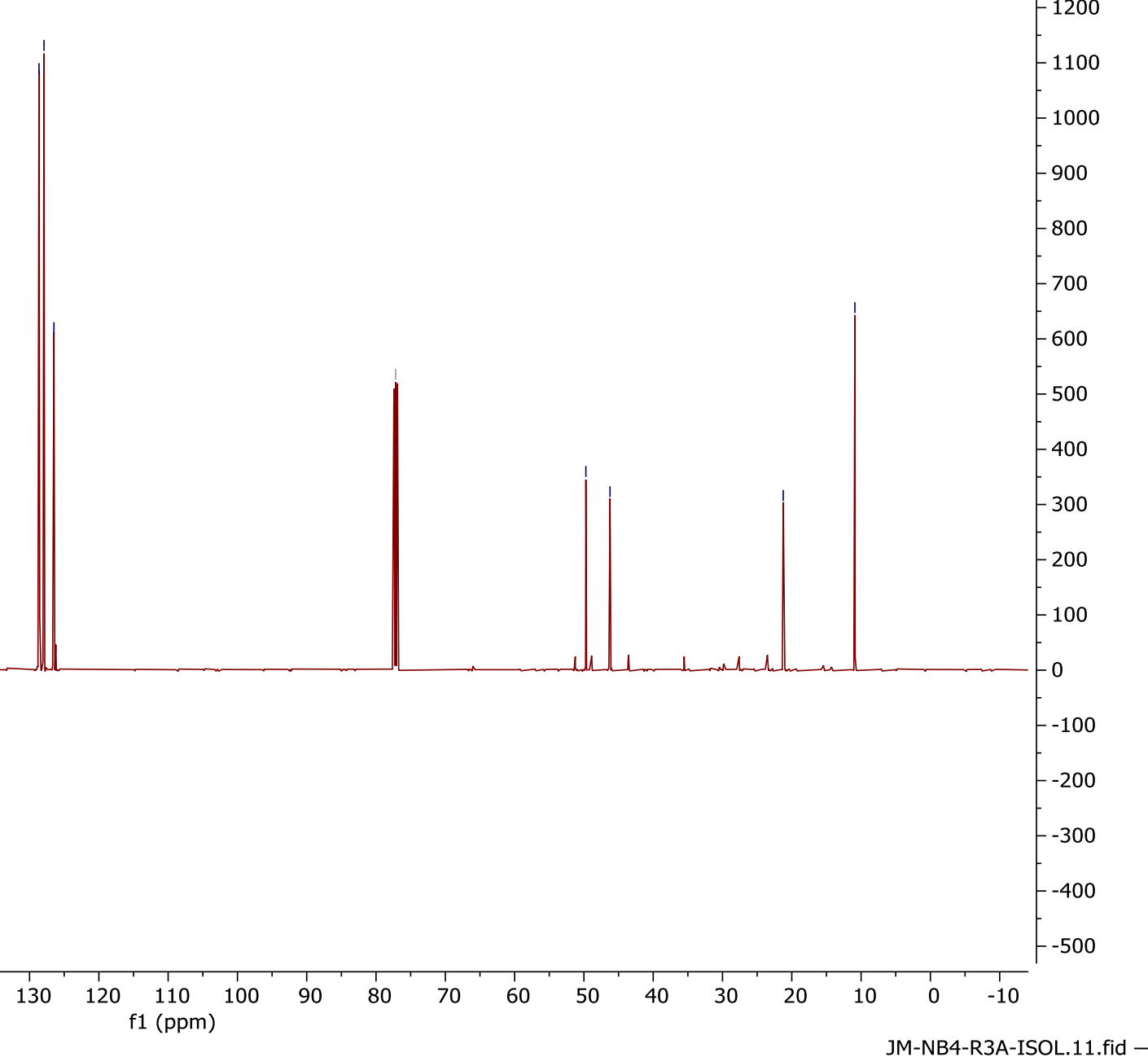

\begin{tabular}{cc} 
& \\
\multirow{2}{*}{} & 8 \\
& 0 \\
\hline
\end{tabular}

-1600
-1500
-1400
-1300
-1200
-1100
-1000
-900
-800
-700
-600
-500
-400
-300
-200
-100
-0
-300
-400
-200
-300
-500 


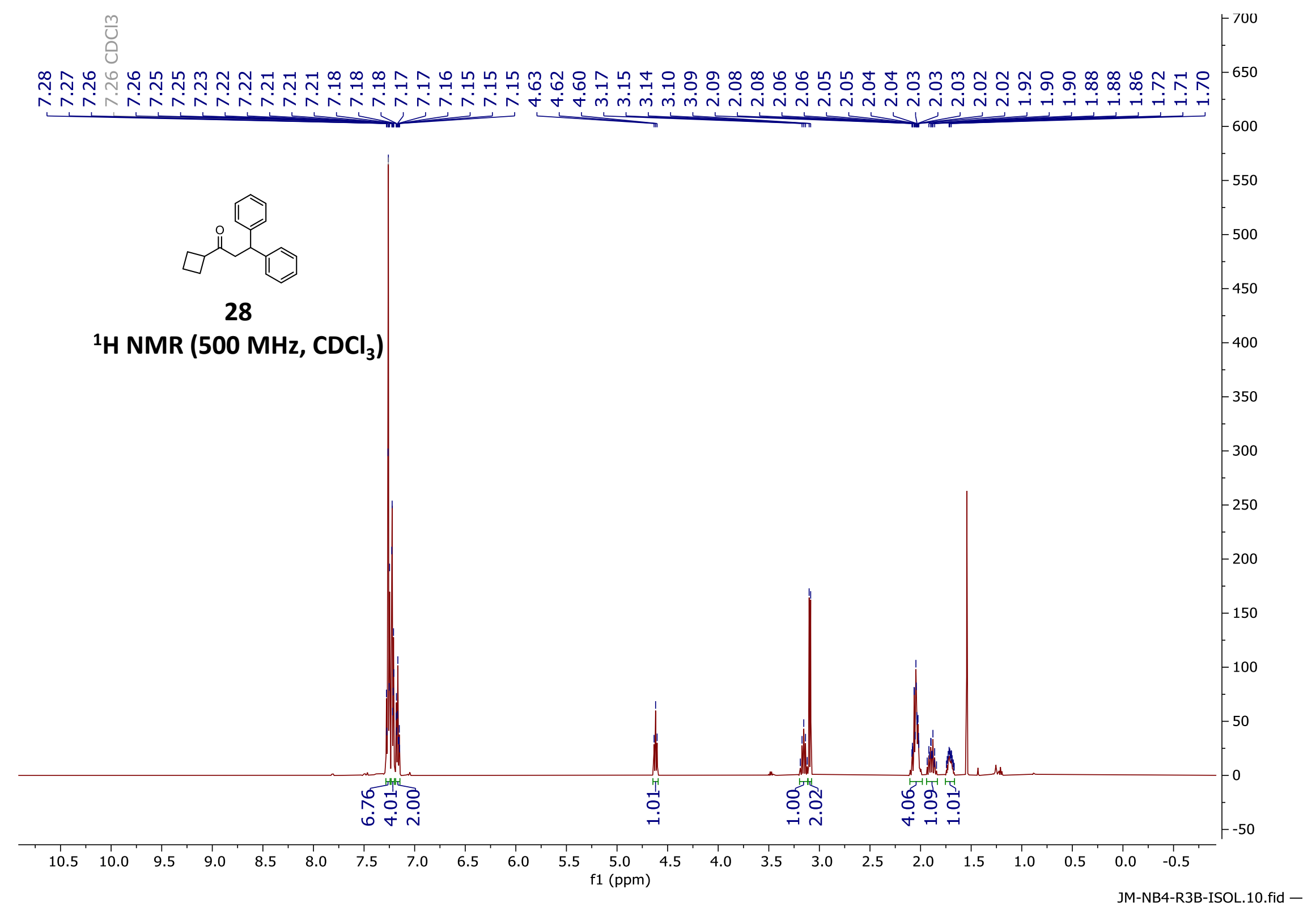




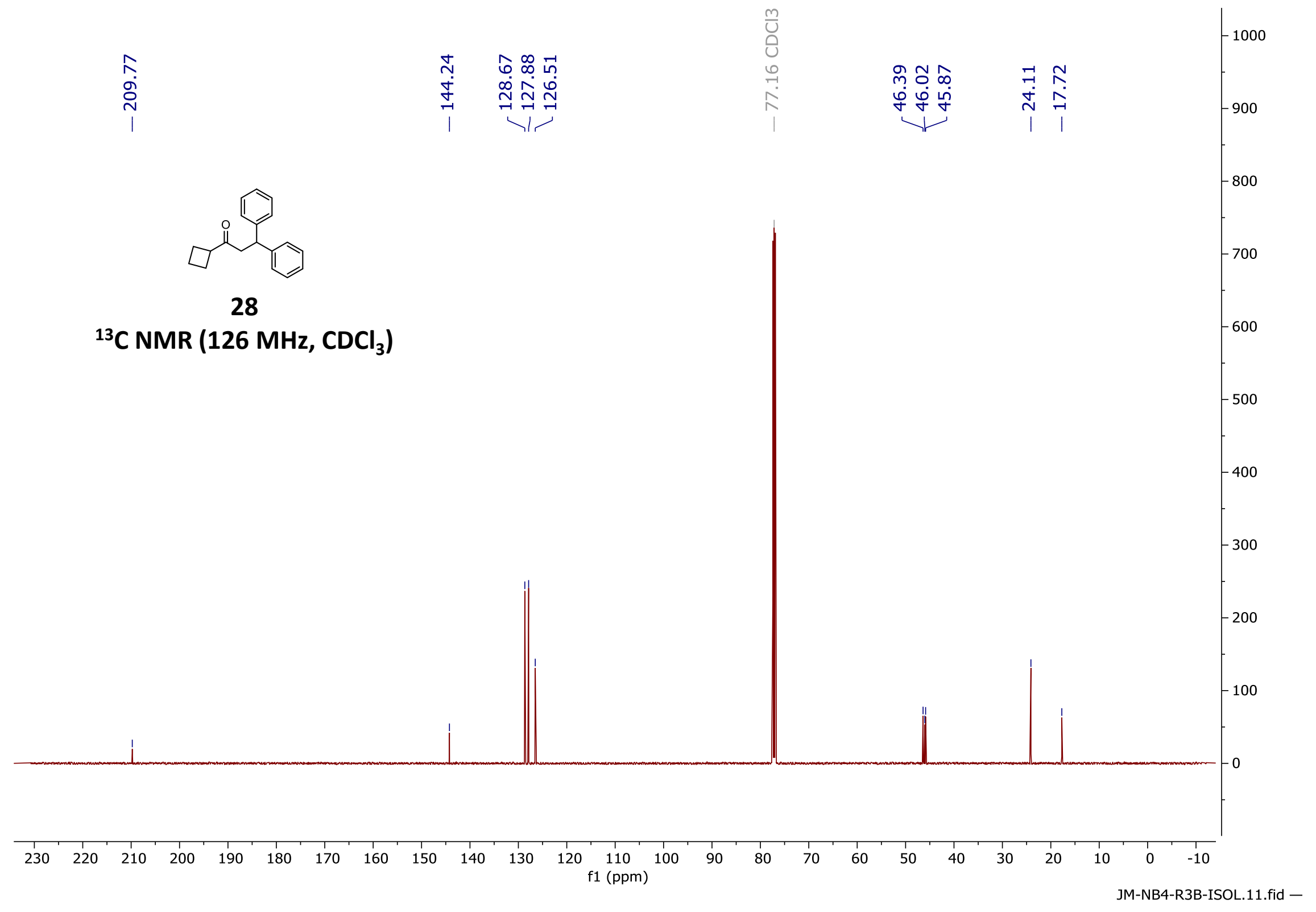




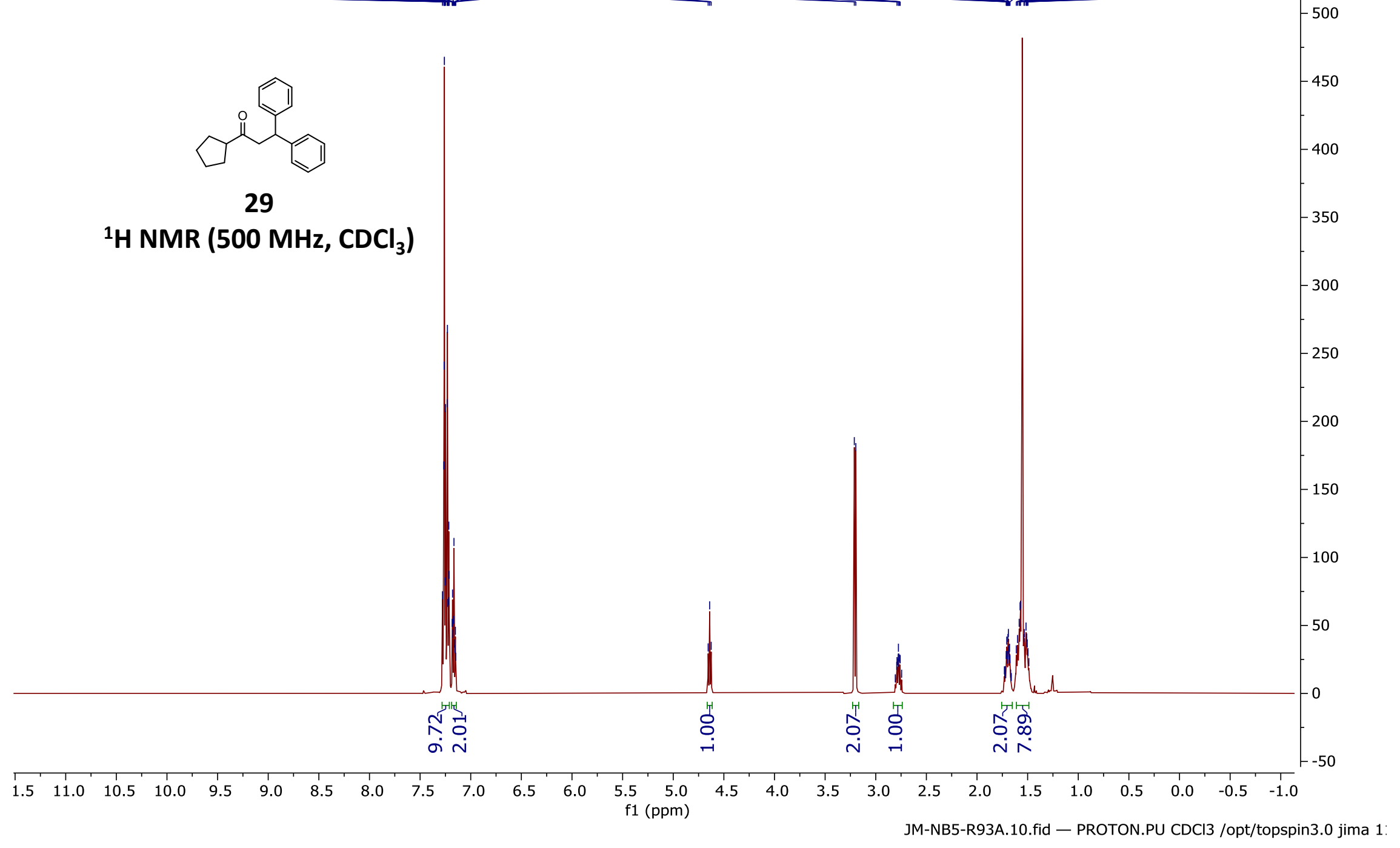




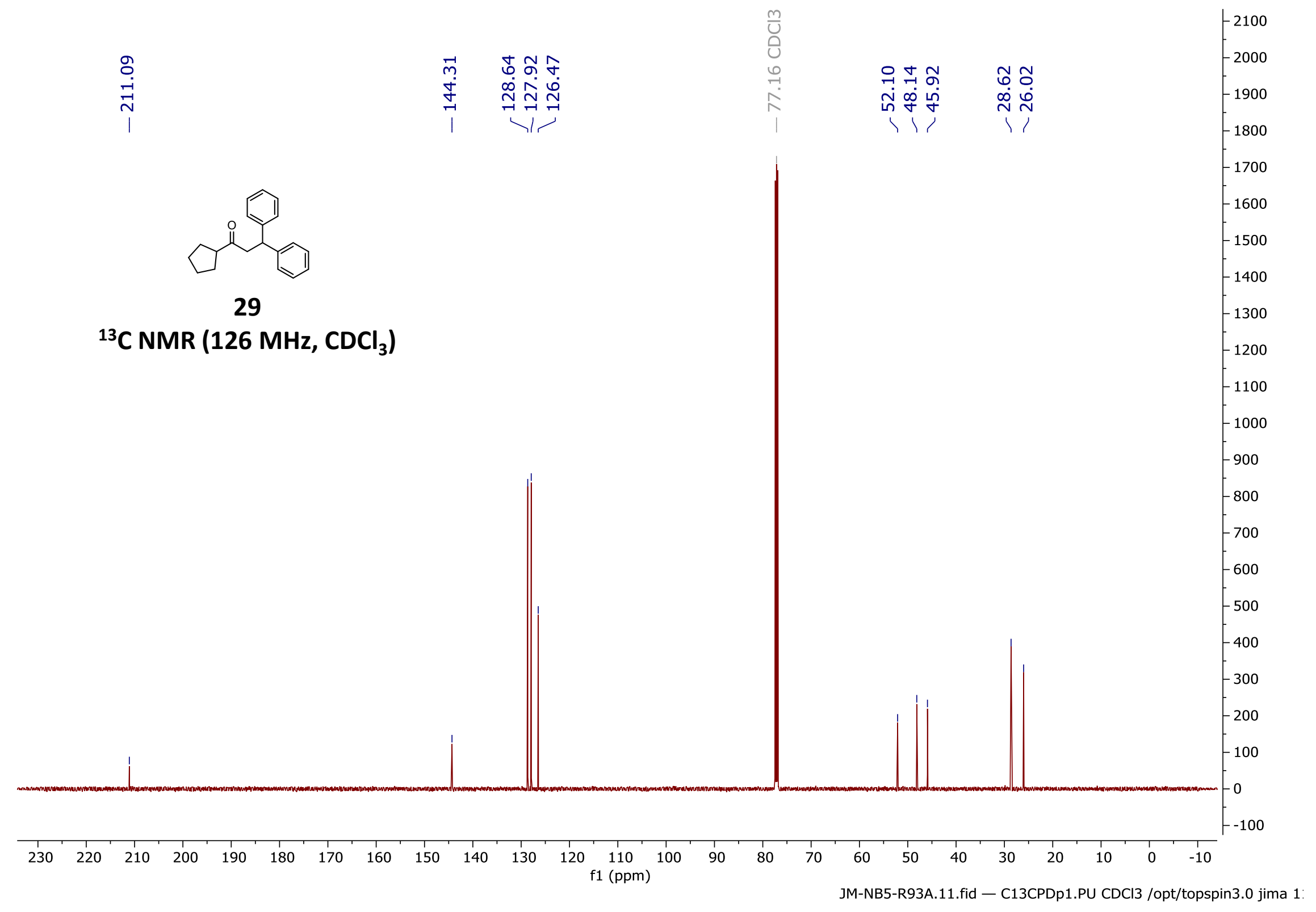




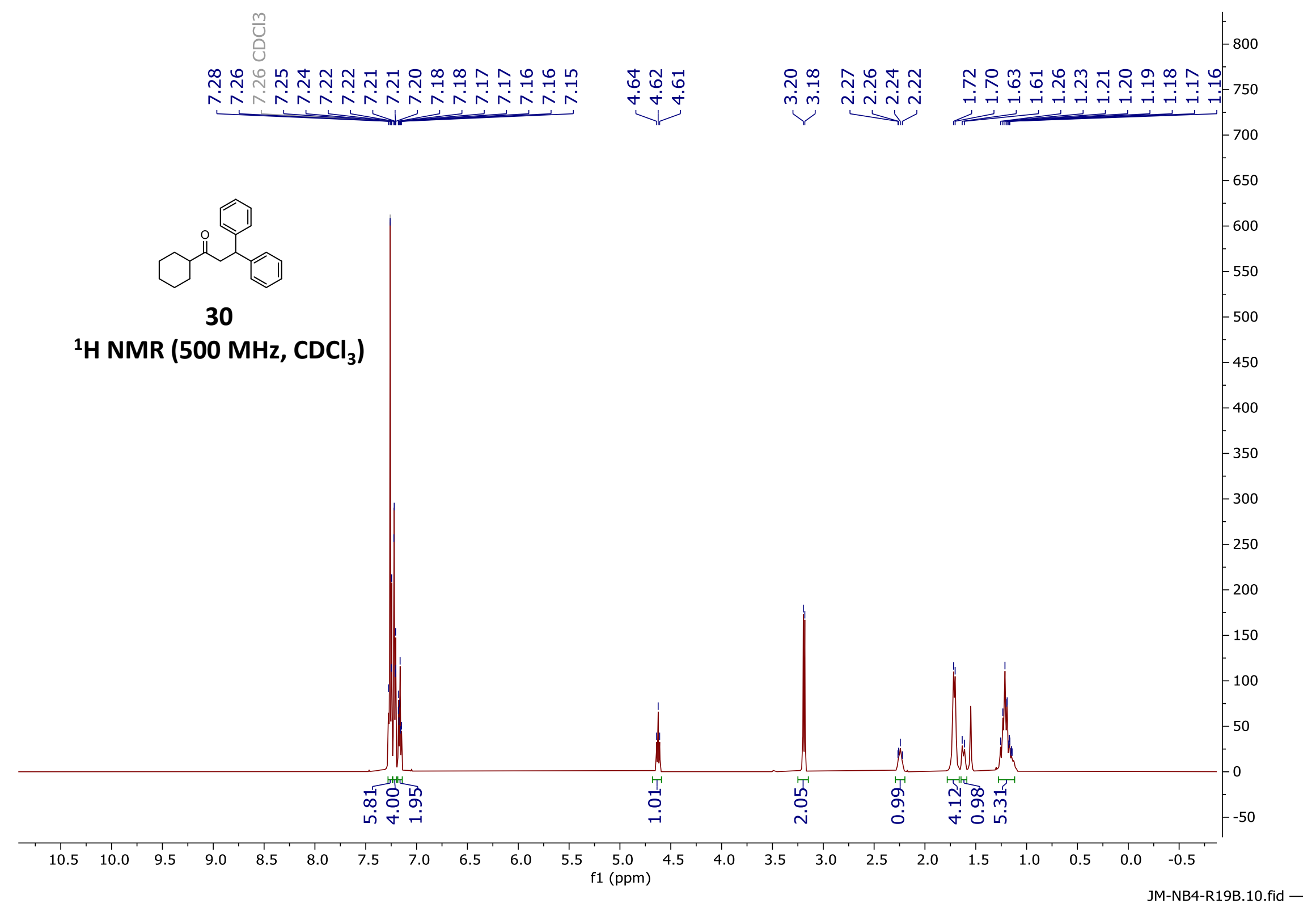




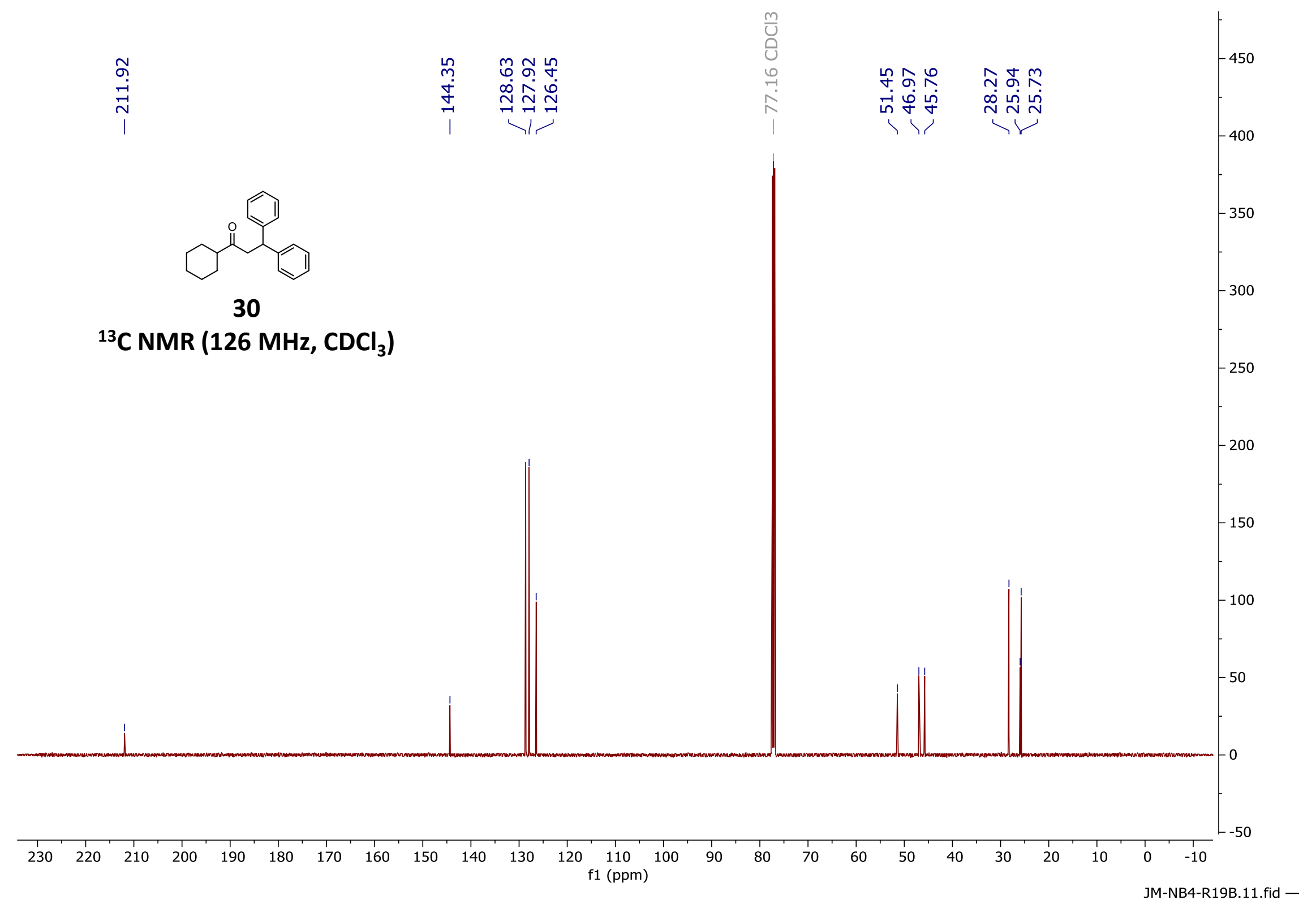




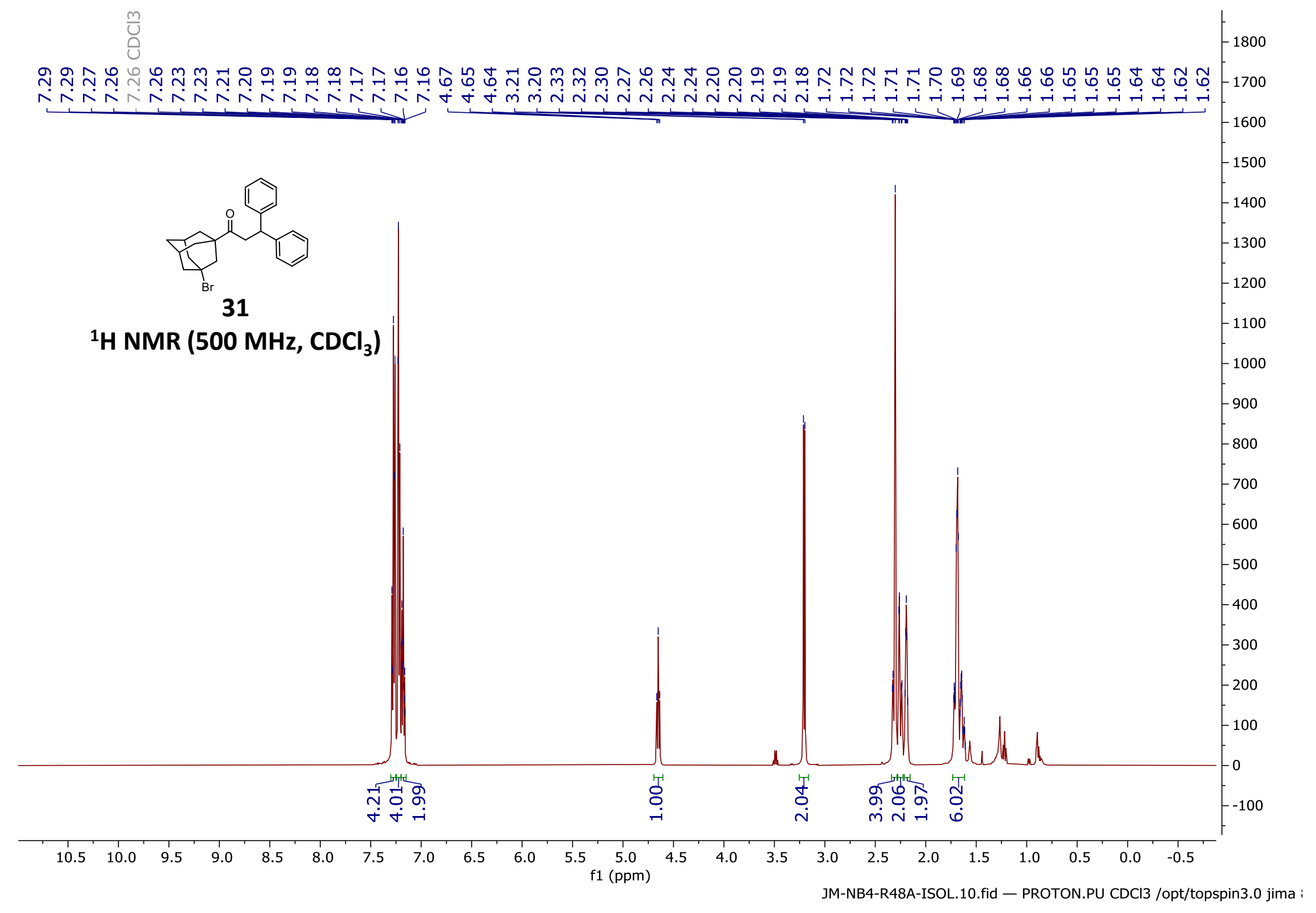




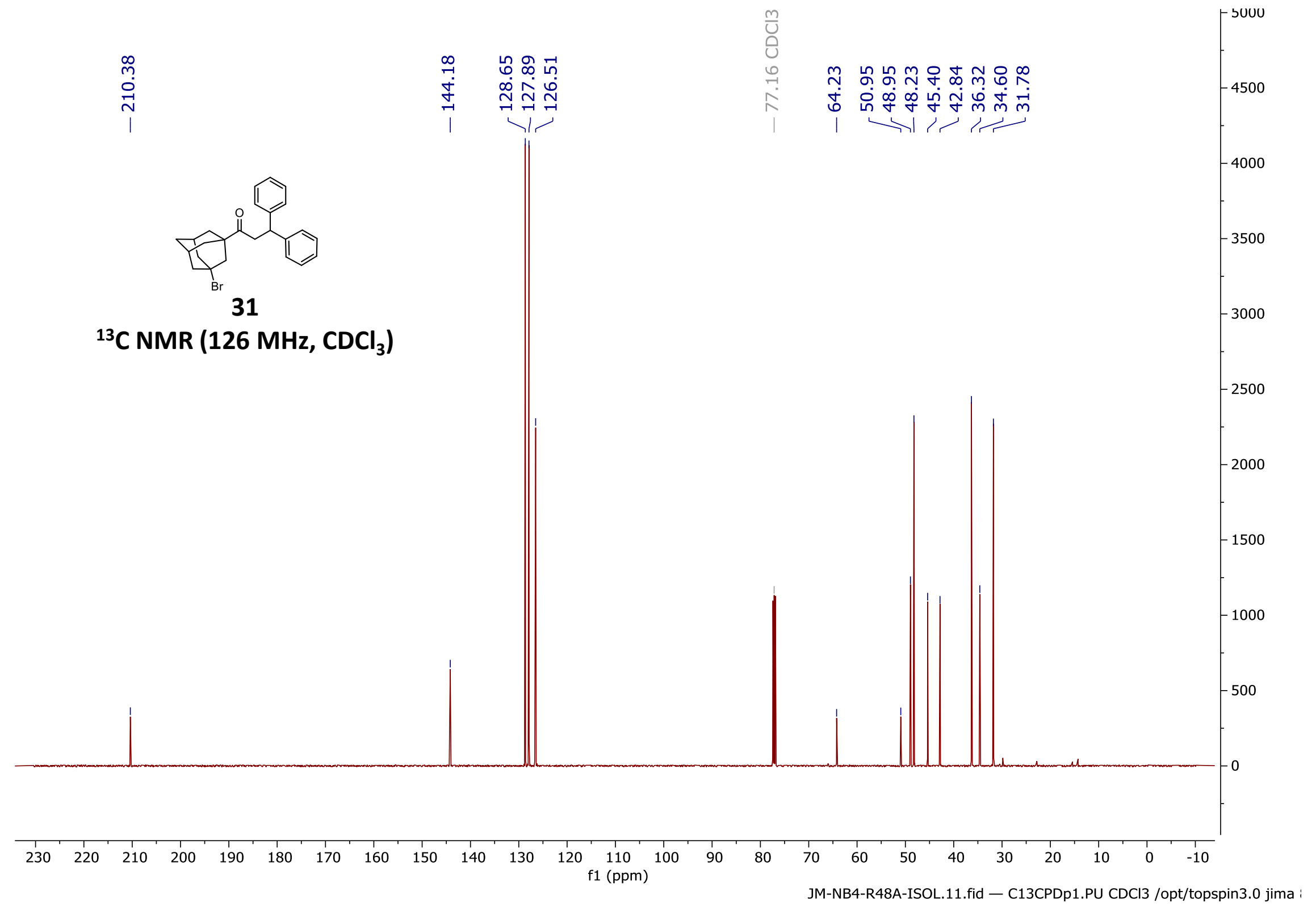




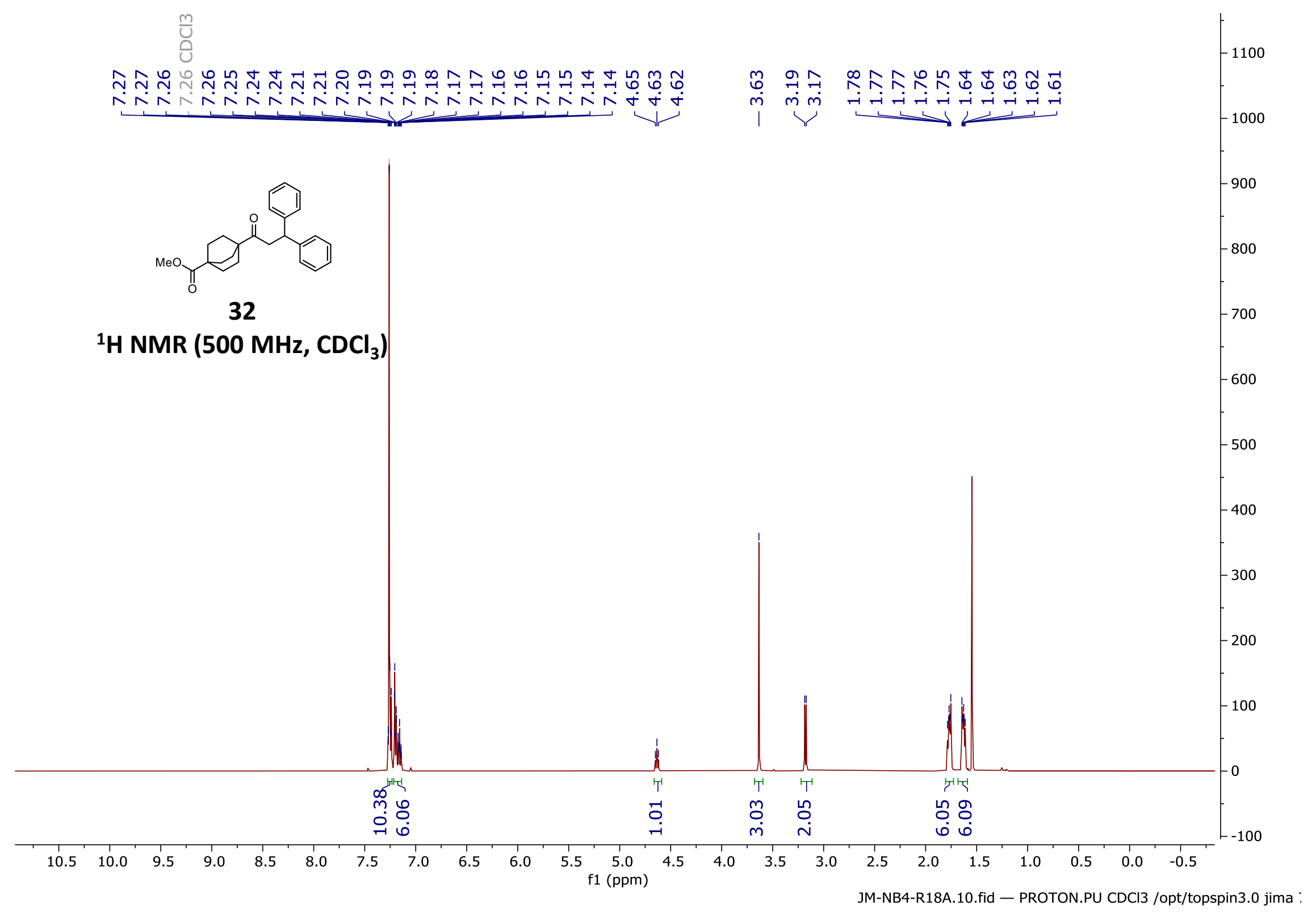




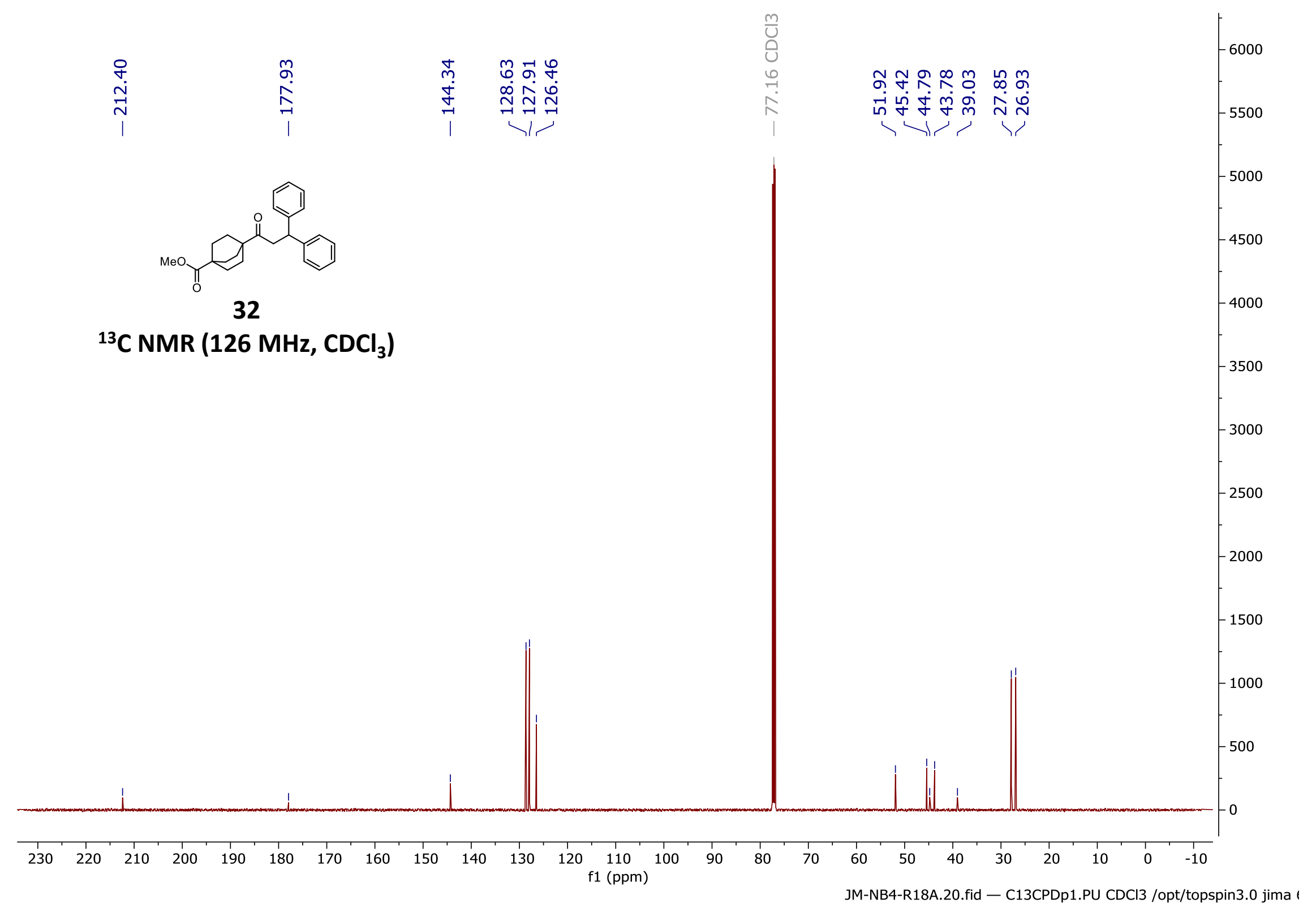




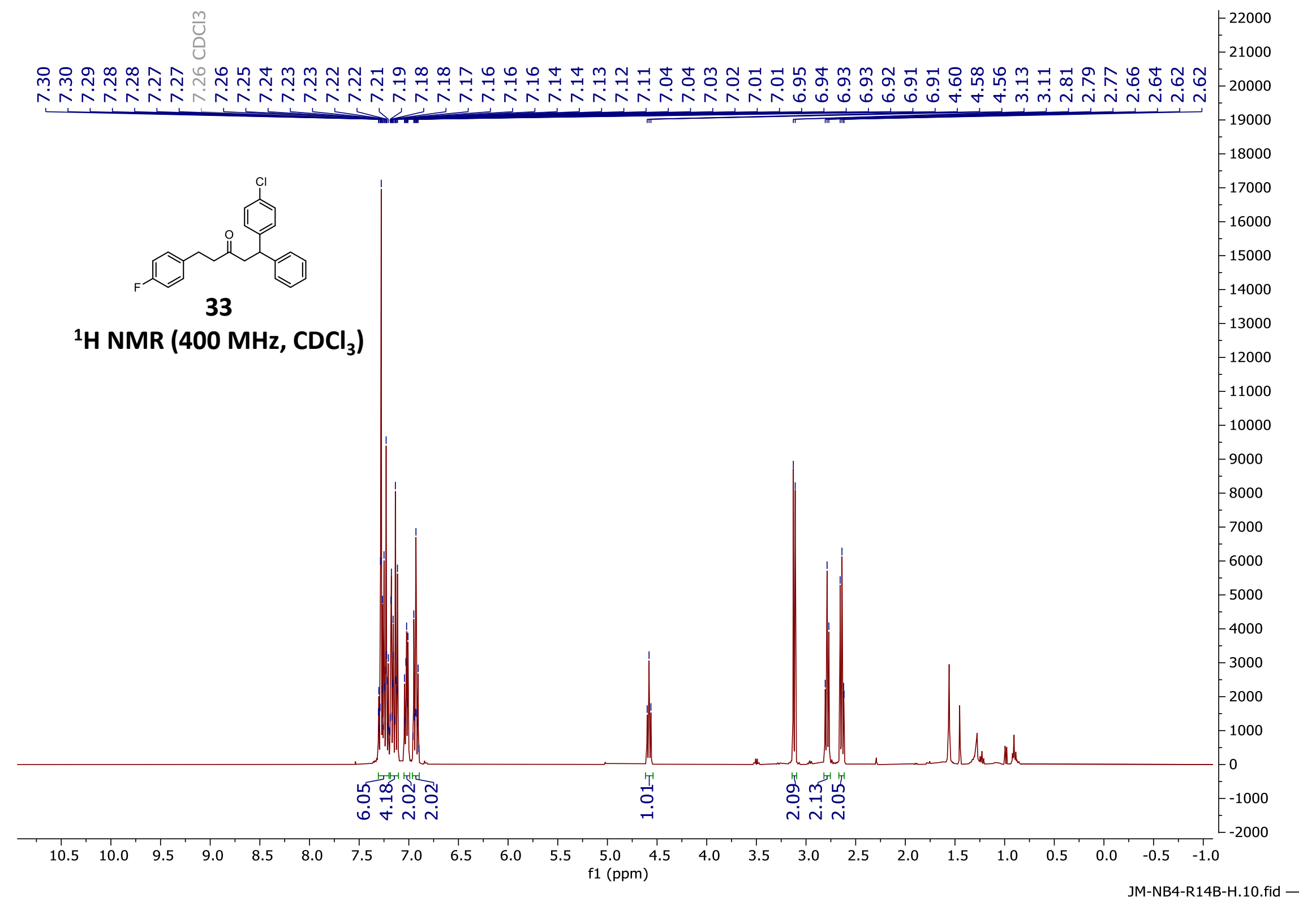




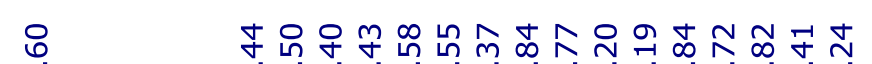

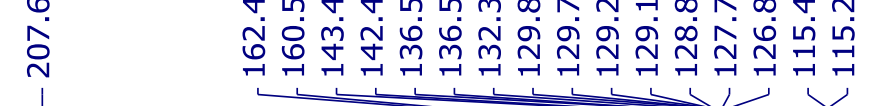

${ }^{13} \mathrm{C} \mathrm{NMR}\left(126 \mathrm{MHz}, \mathrm{CDCl}_{3}\right)$

守守守

$\stackrel{\substack{\infty \\ \infty}}{\infty}$

$-2400$

2300

2100

2000

1900

1800

$-1700$

1600

$-1500$

1300

1200

1100

1000

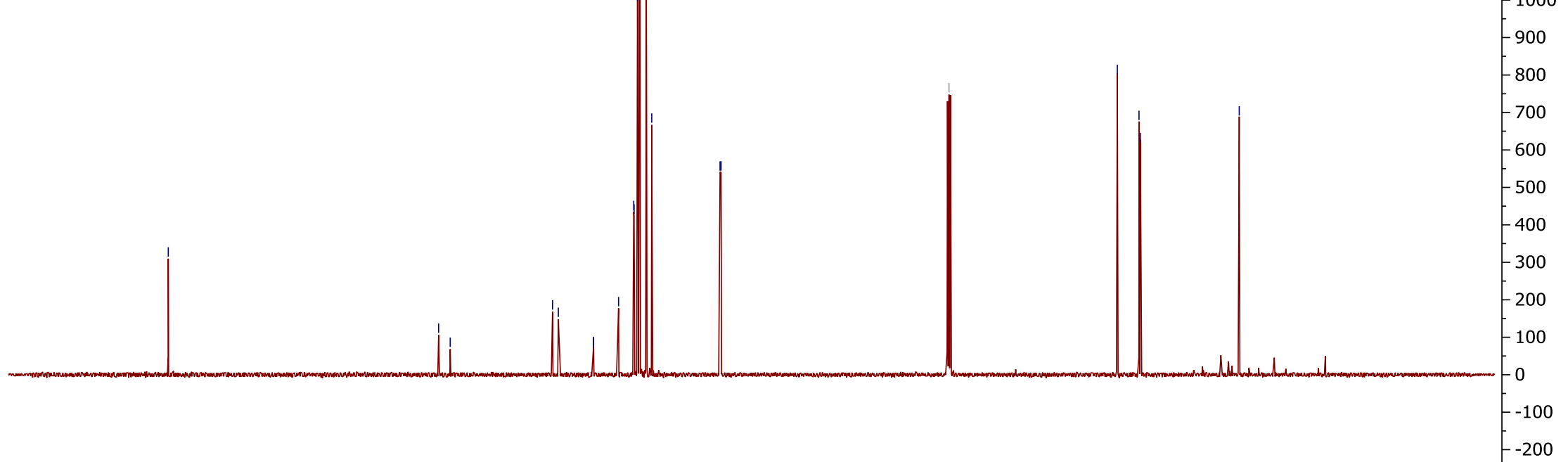

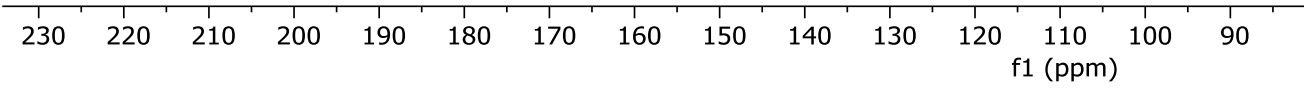

JM-NB4-R14B.11.fid - C13CPDp1.PU CDCl3 /opt/topspin3.0 jima 


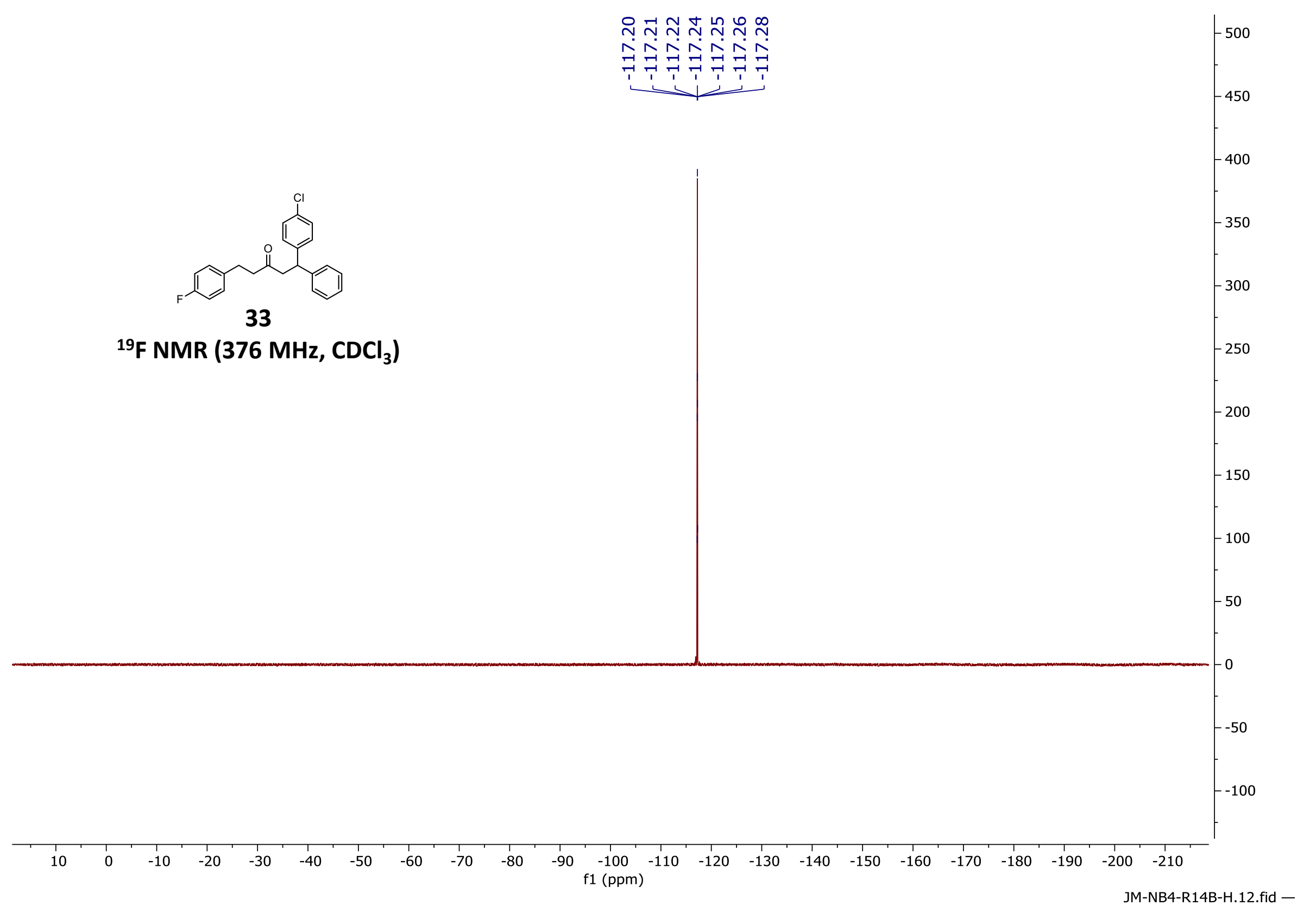




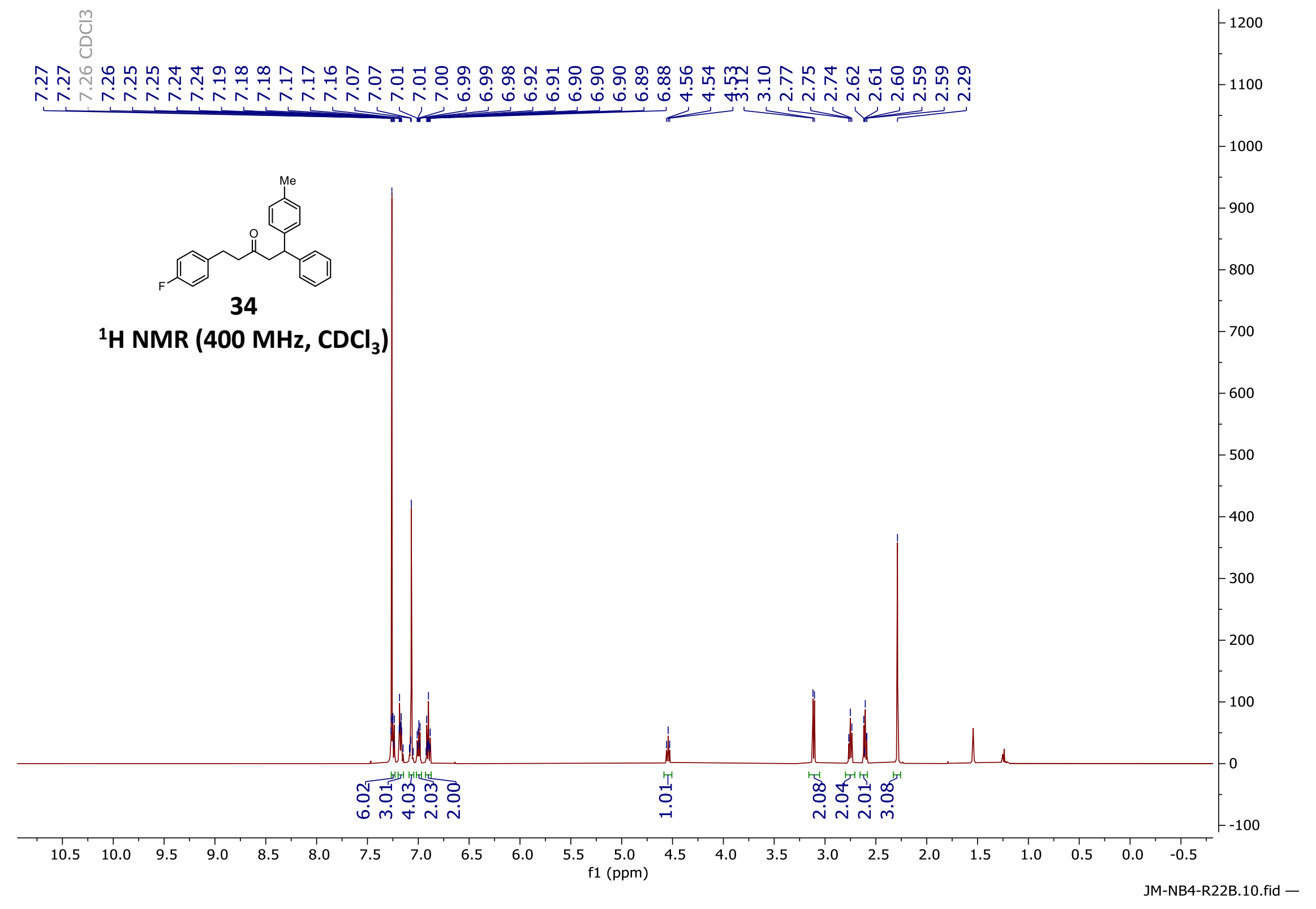


6.

$\stackrel{\infty}{\sim}$

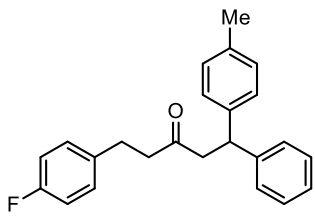

34

${ }^{13} \mathrm{C}$ NMR (126 MHz, $\left.\mathrm{CDCl}_{3}\right)$

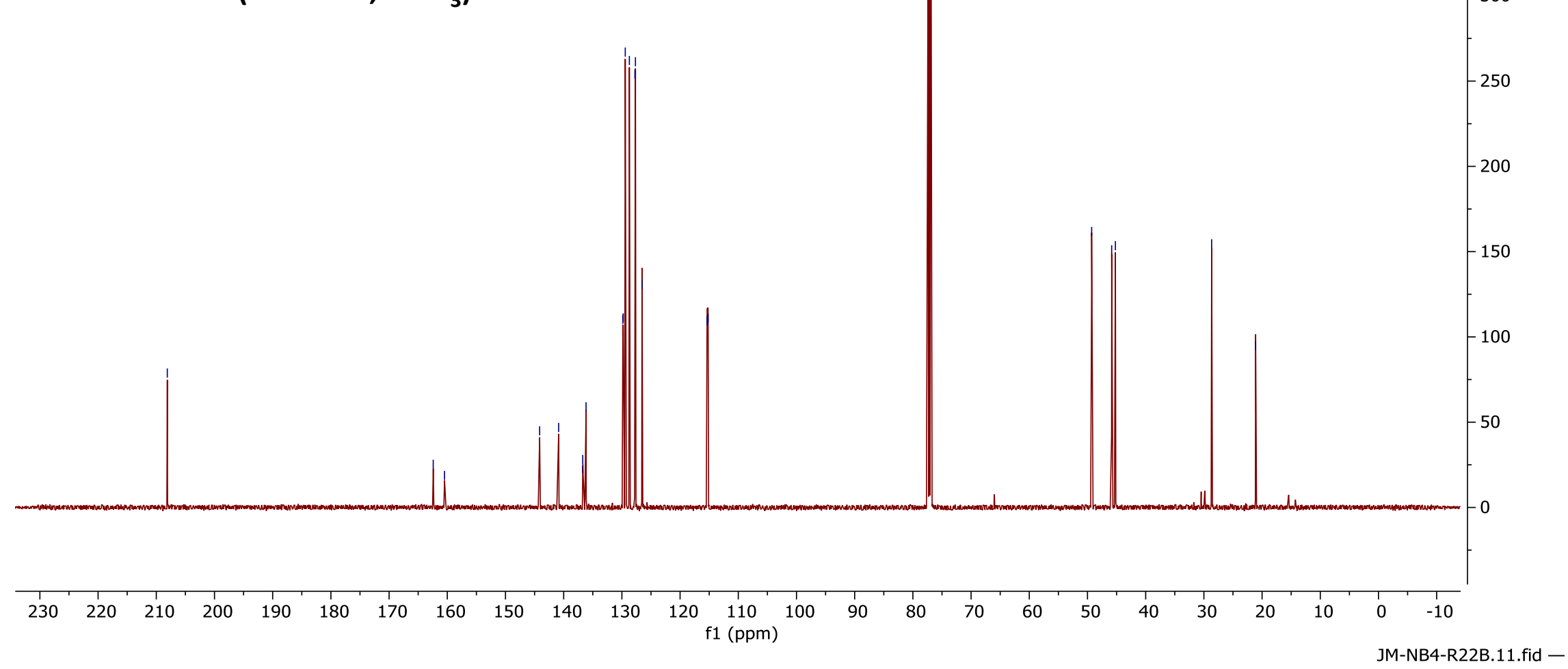




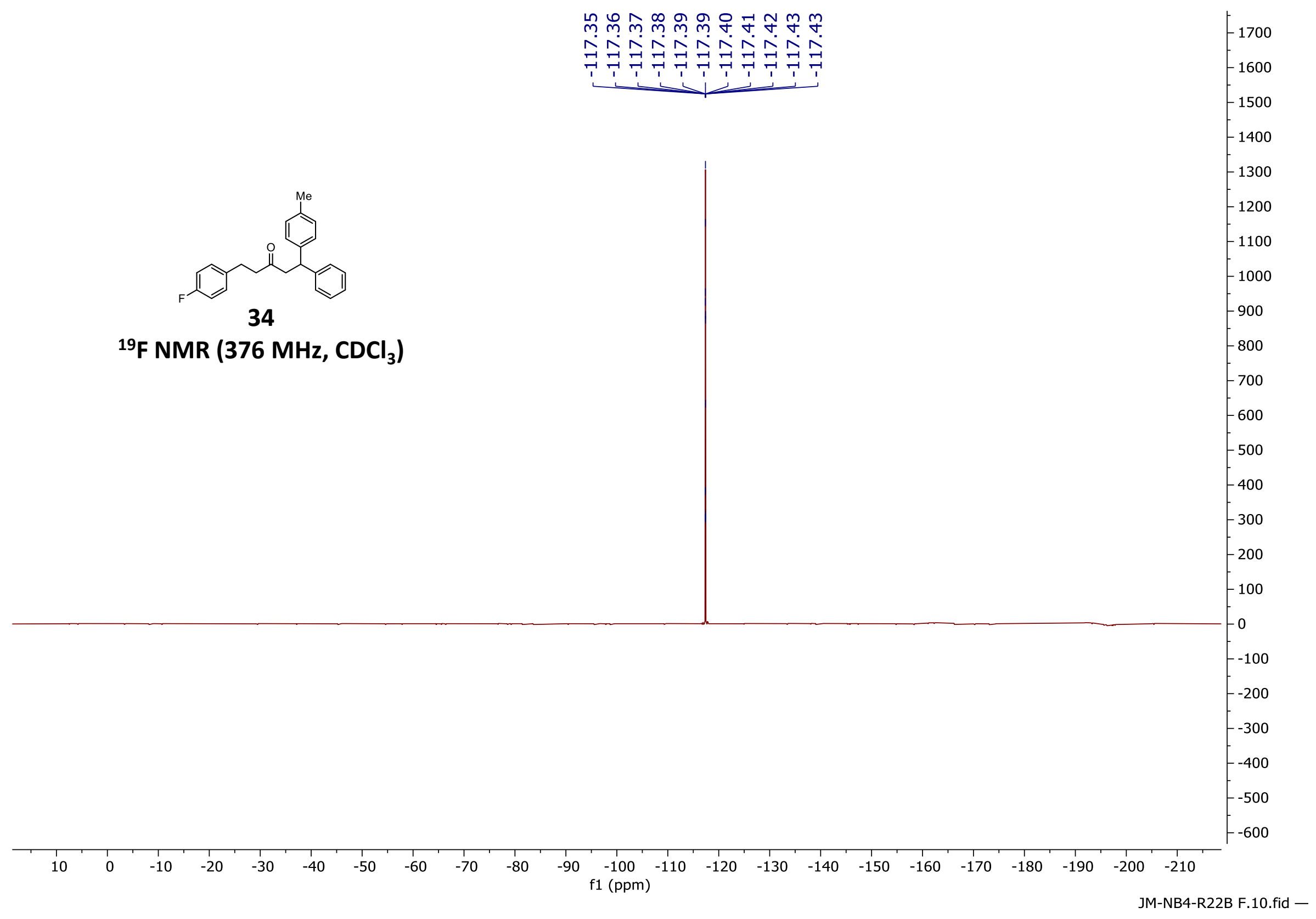




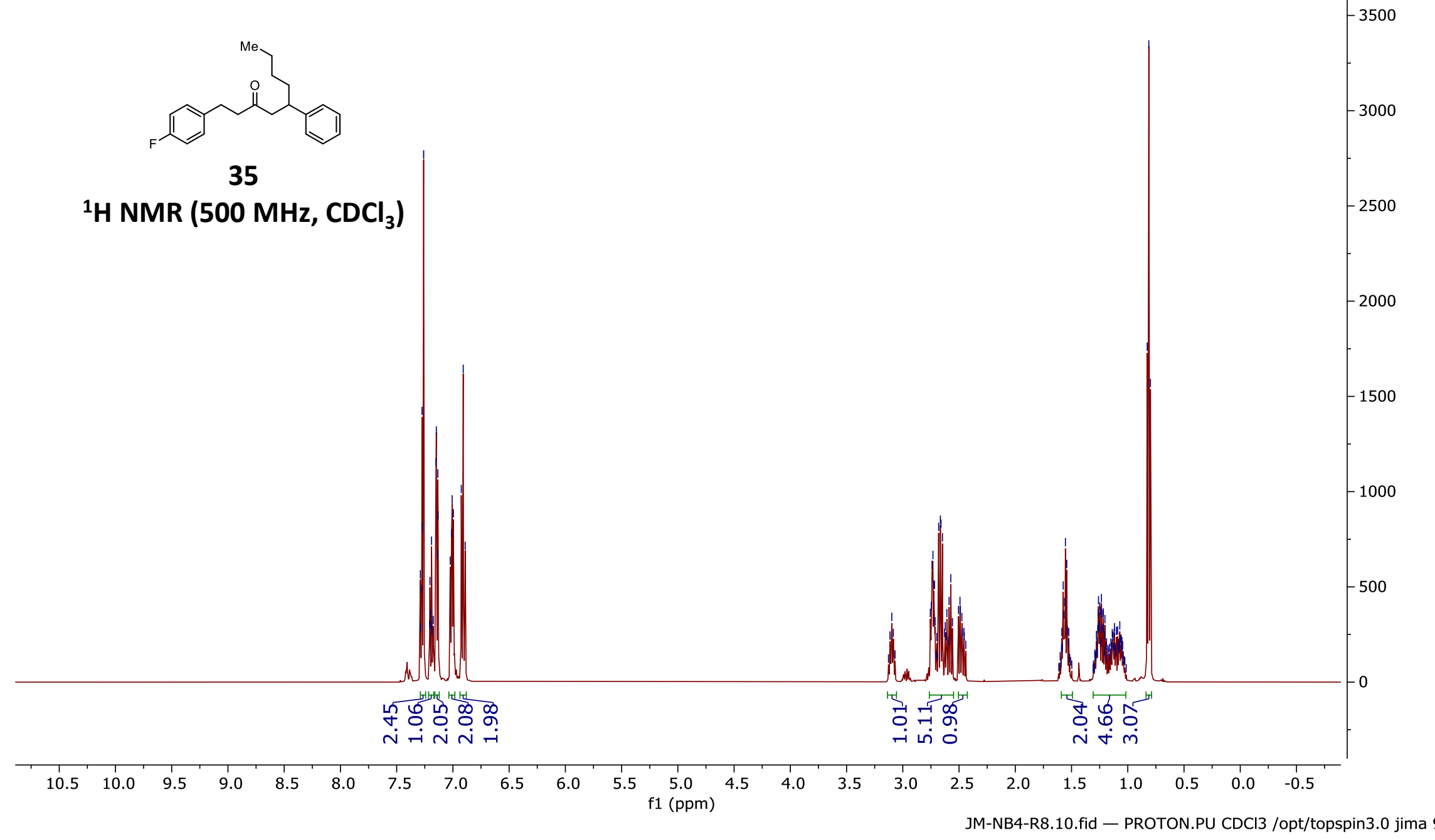




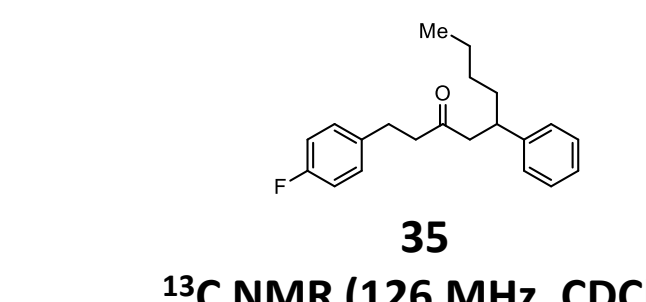

${ }^{13} \mathrm{C}$ NMR (126 MHz, $\mathrm{CDCl}_{3}$ )

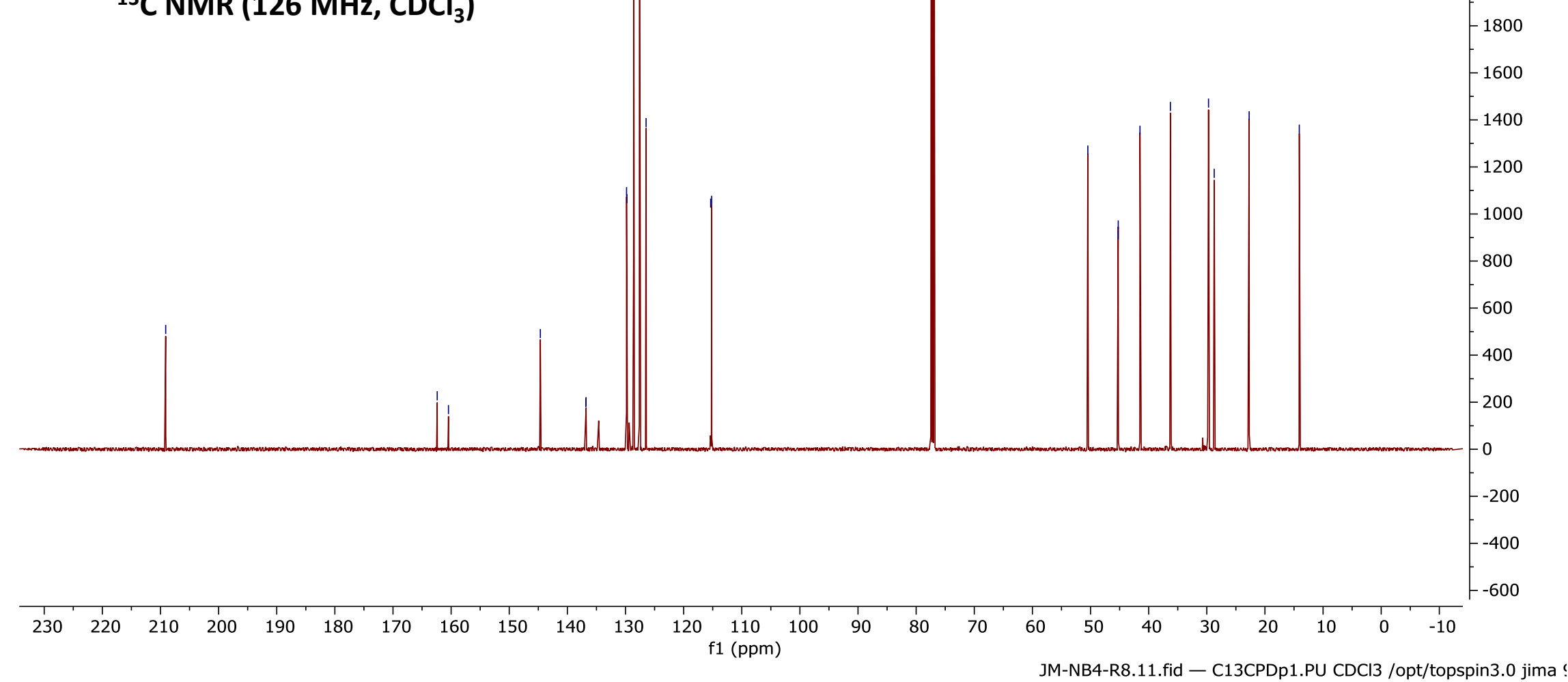




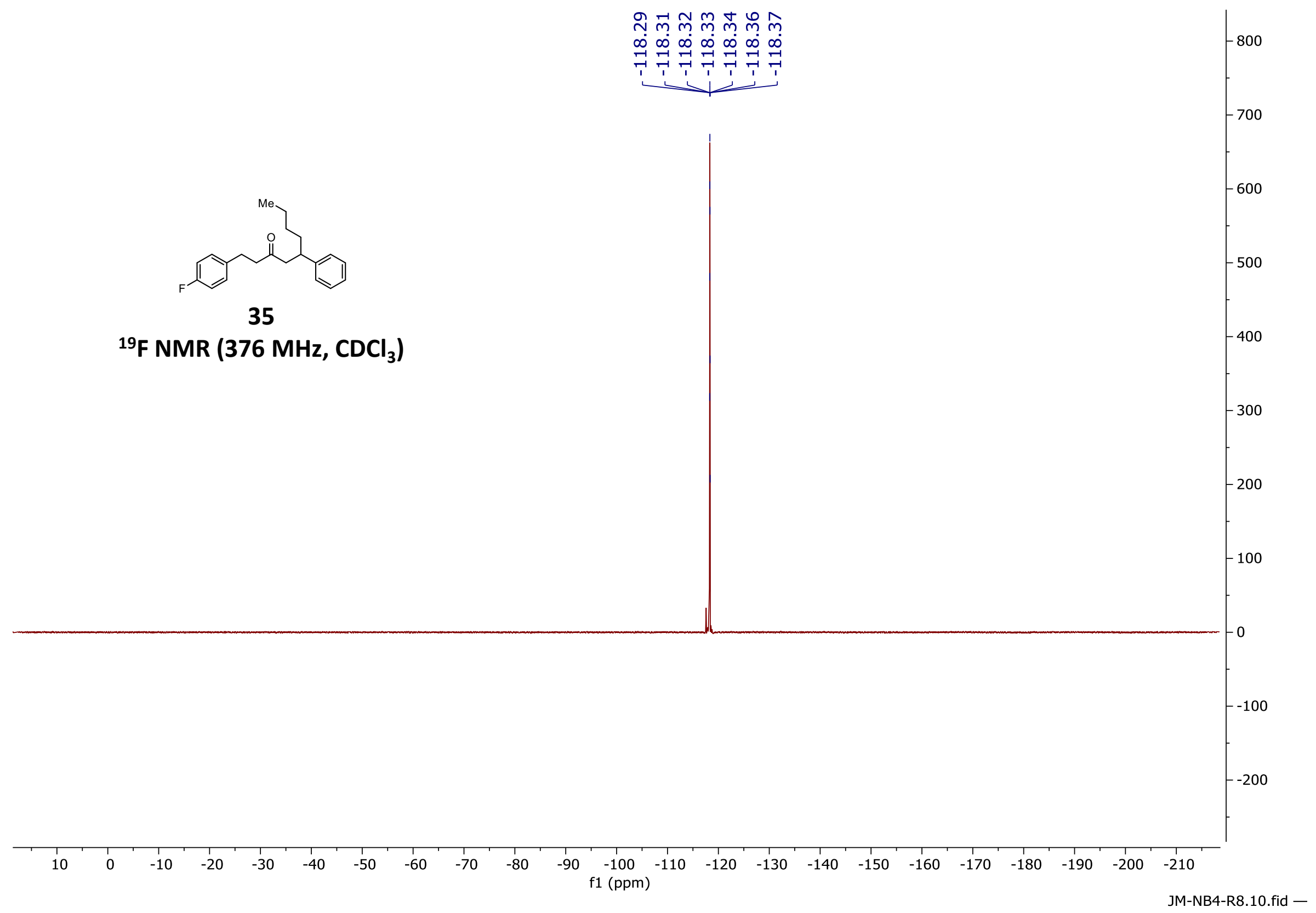


<smiles>COc1ccc2c(c1)CC(CC(=O)Cc1ccc(F)cc1)CCC2</smiles>

36

${ }^{1} \mathrm{H}$ NMR $\left(500 \mathrm{MHz}, \mathrm{CDCl}_{3}\right)$

3500

3000

2500

$-2000$

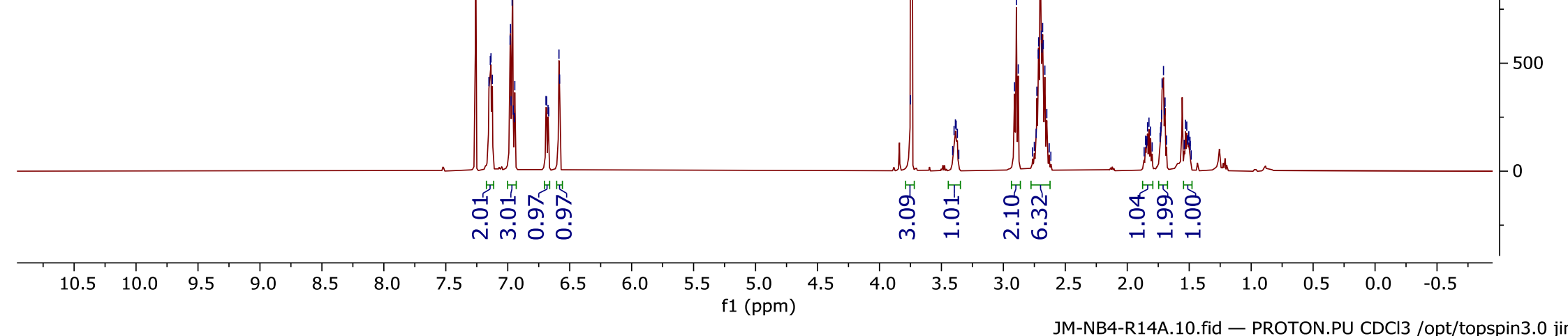

JM-NB4-R14A.10.fid - PROTON.PU CDCl3 /opt/topspin3.0 jima । 


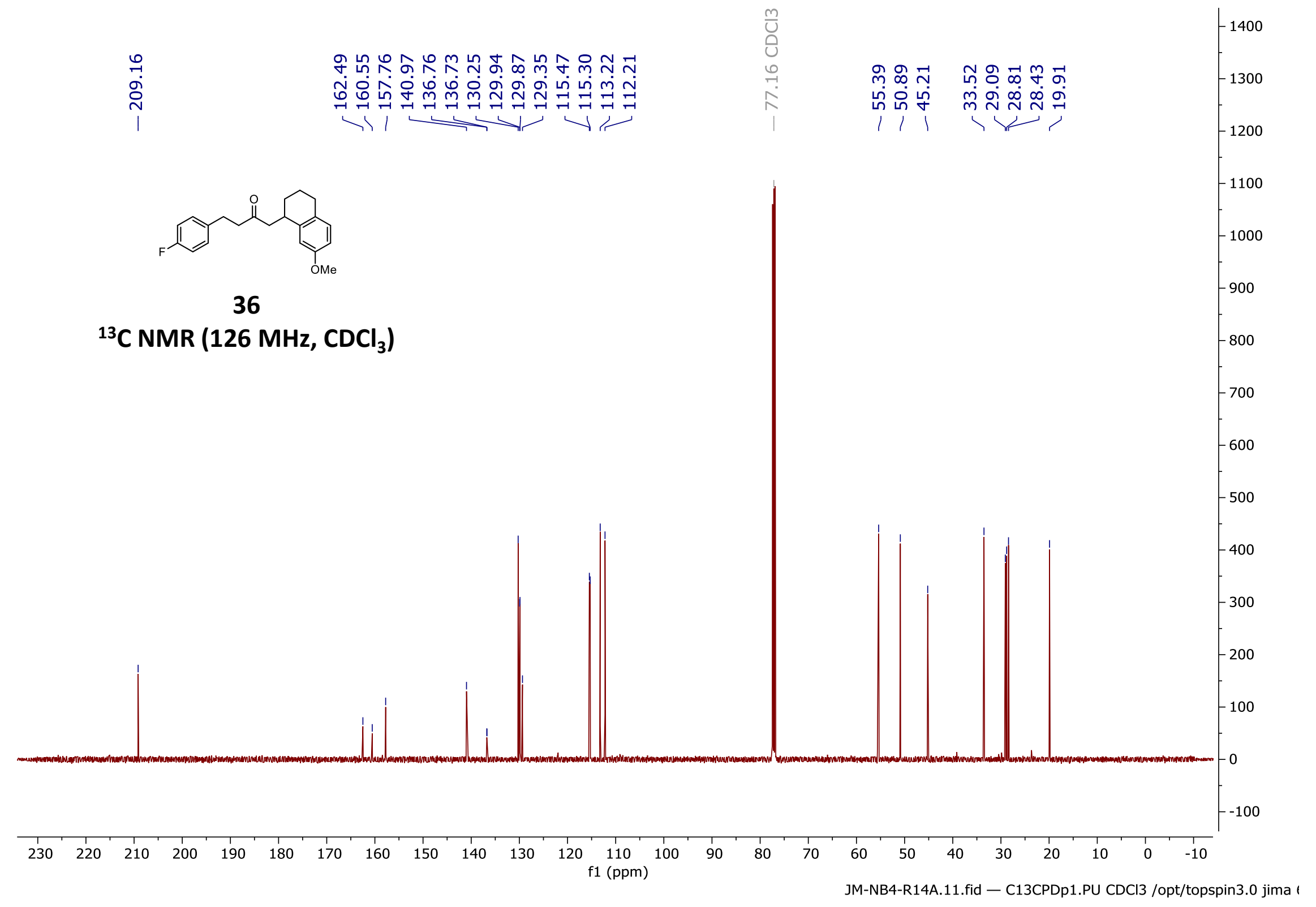




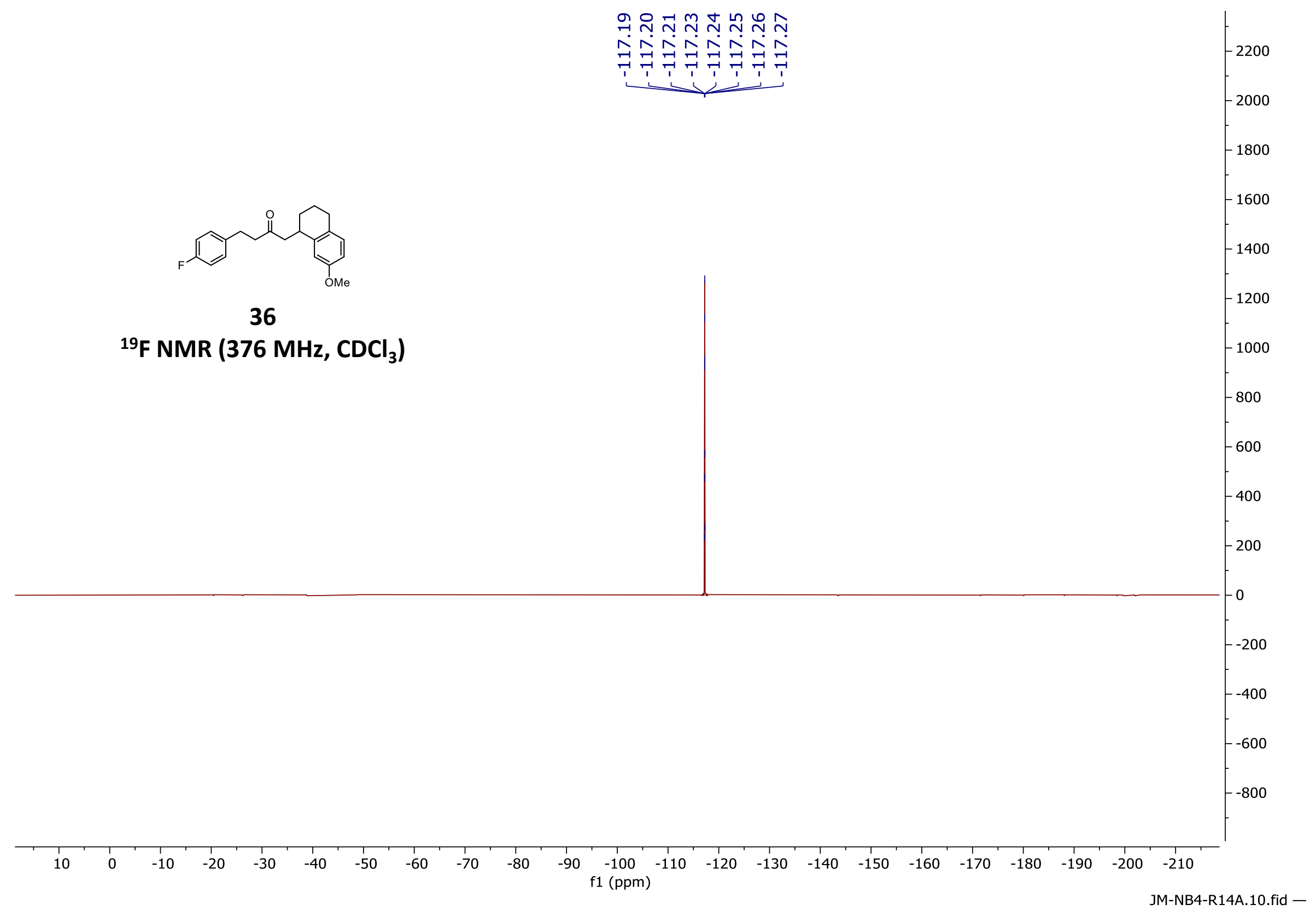




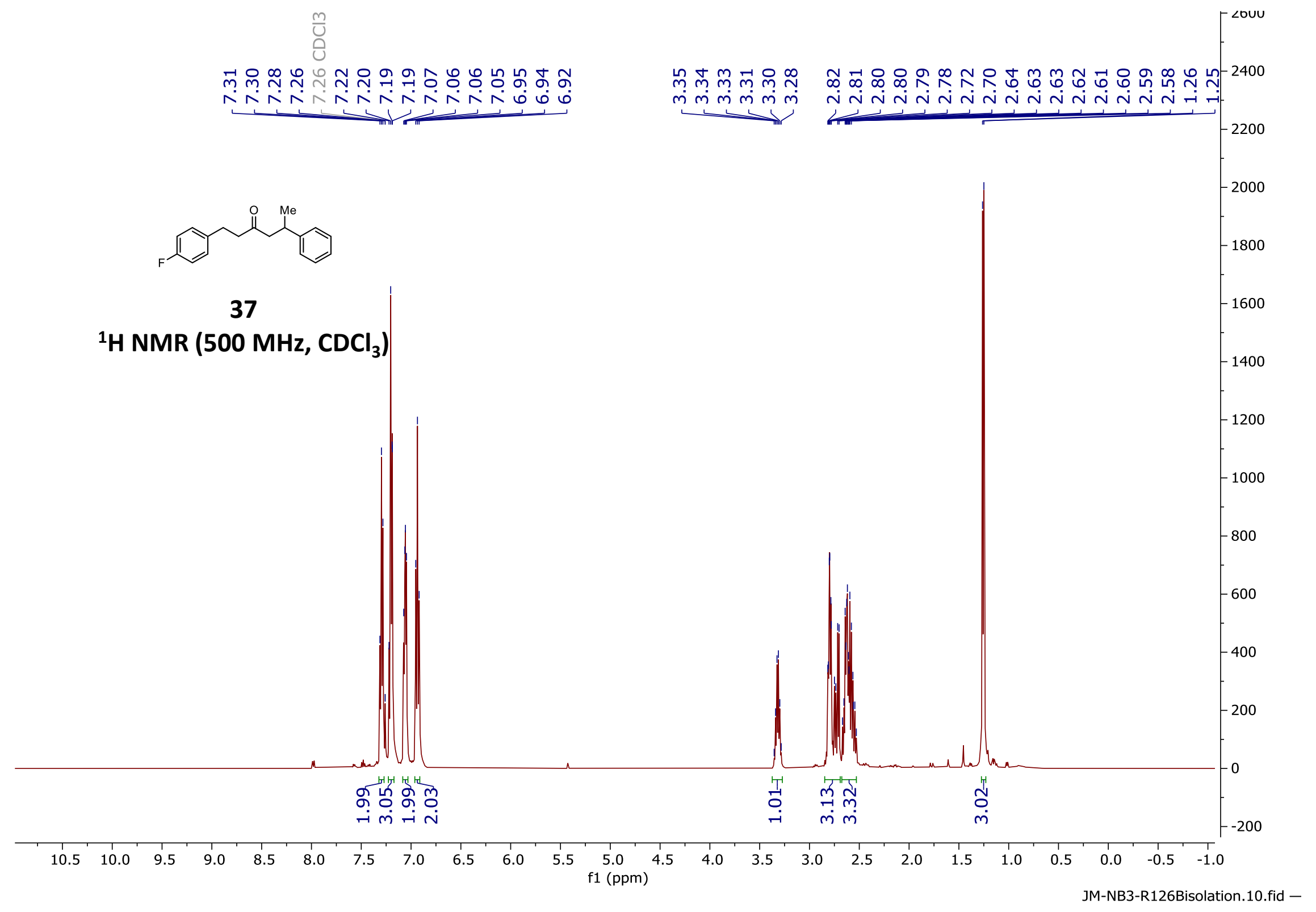




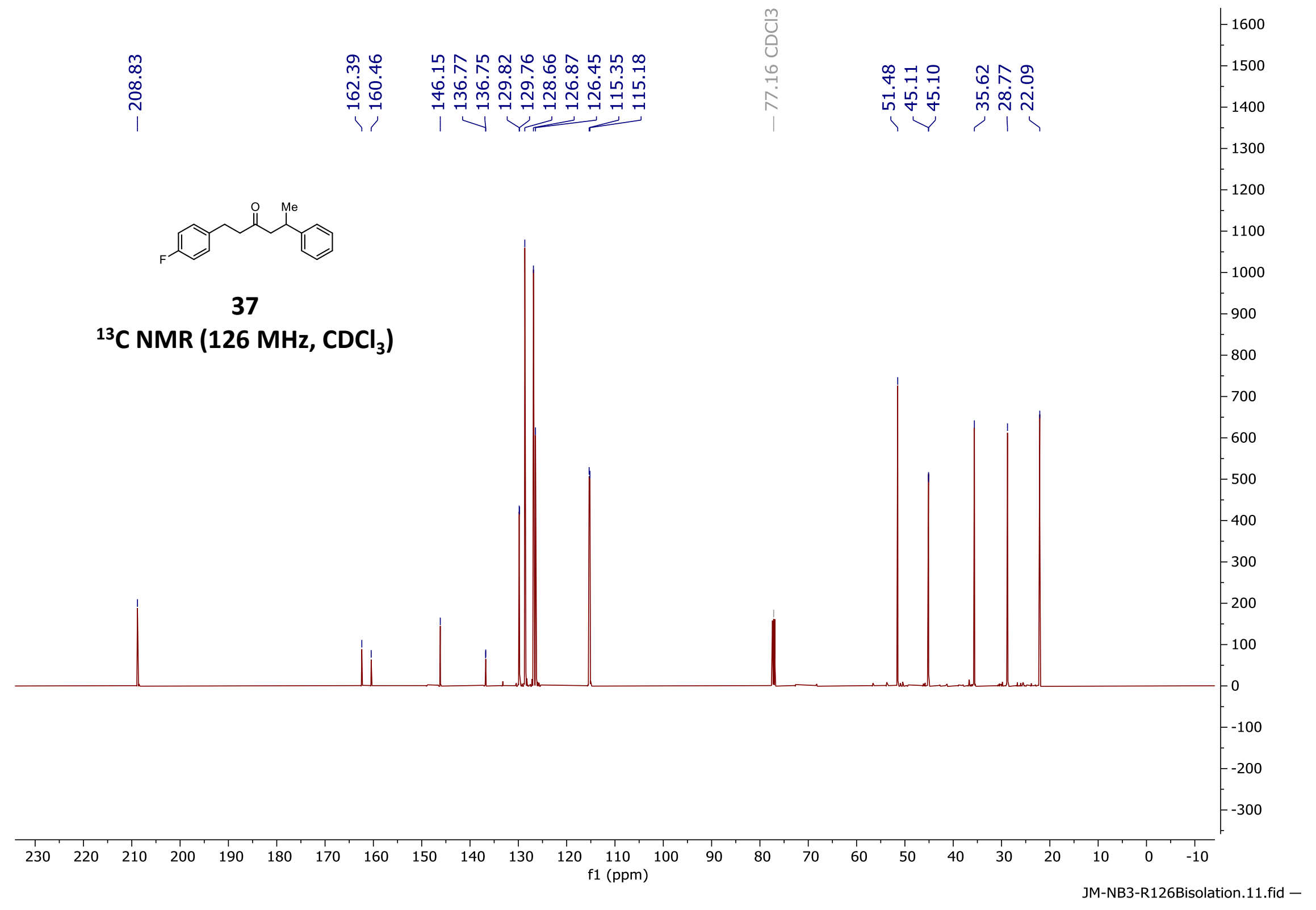




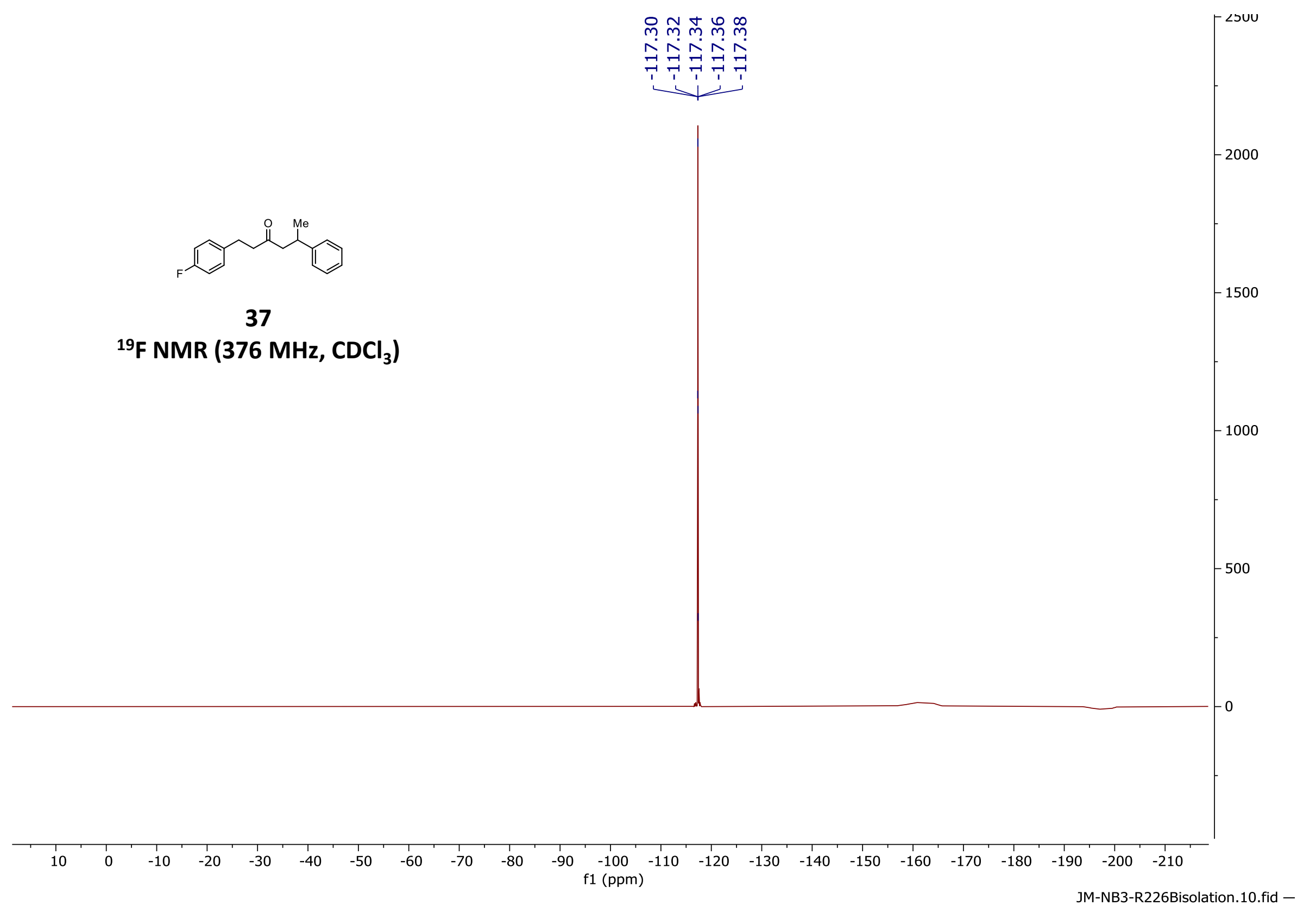


m

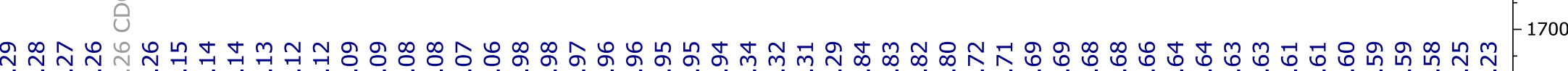

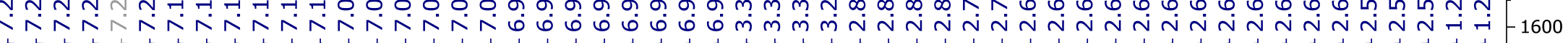

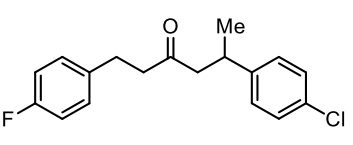

38

${ }^{1} \mathrm{H}$ NMR $\left.\left(500 \mathrm{MHz}^{\mathrm{CDCl}}\right)_{3}\right)$

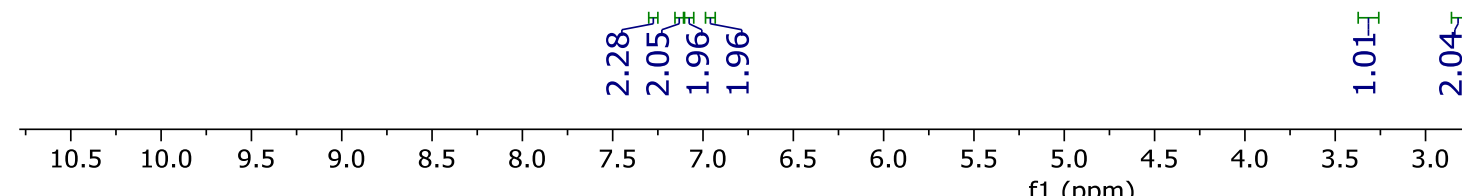




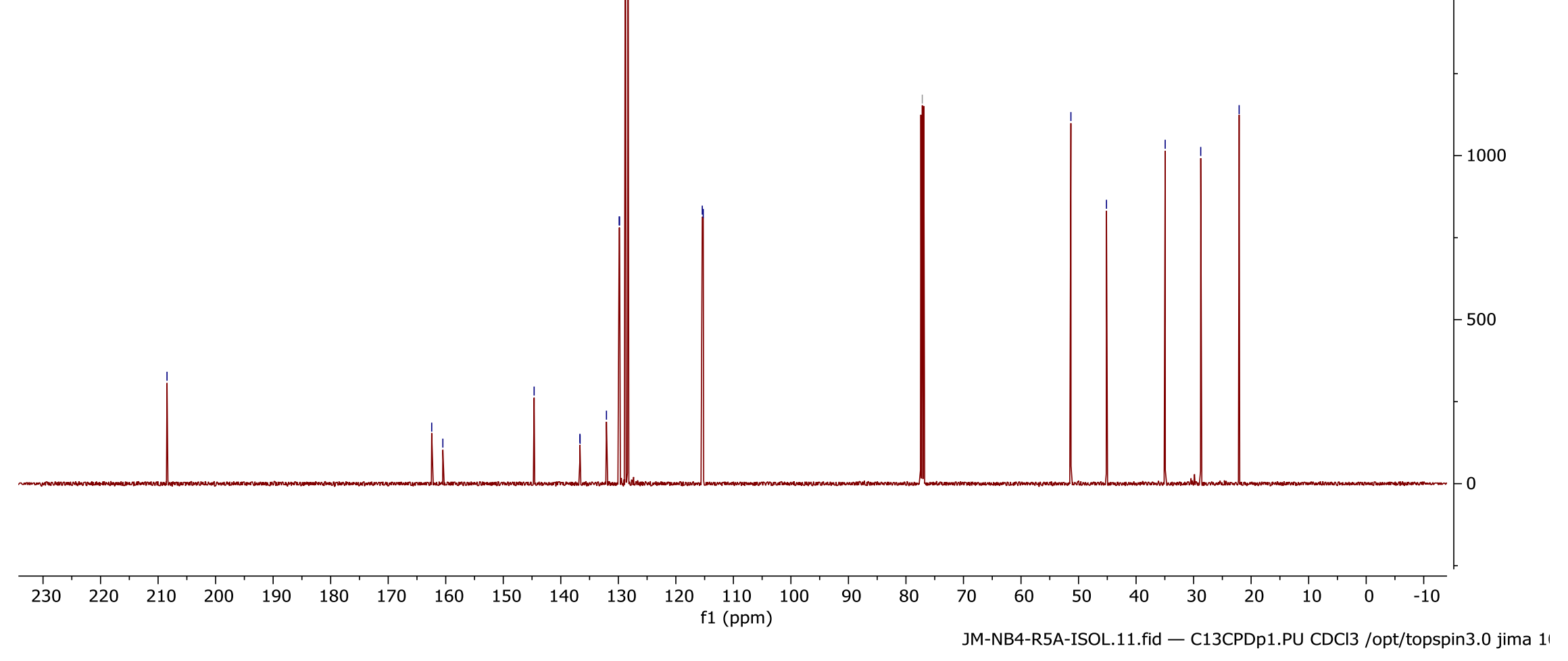




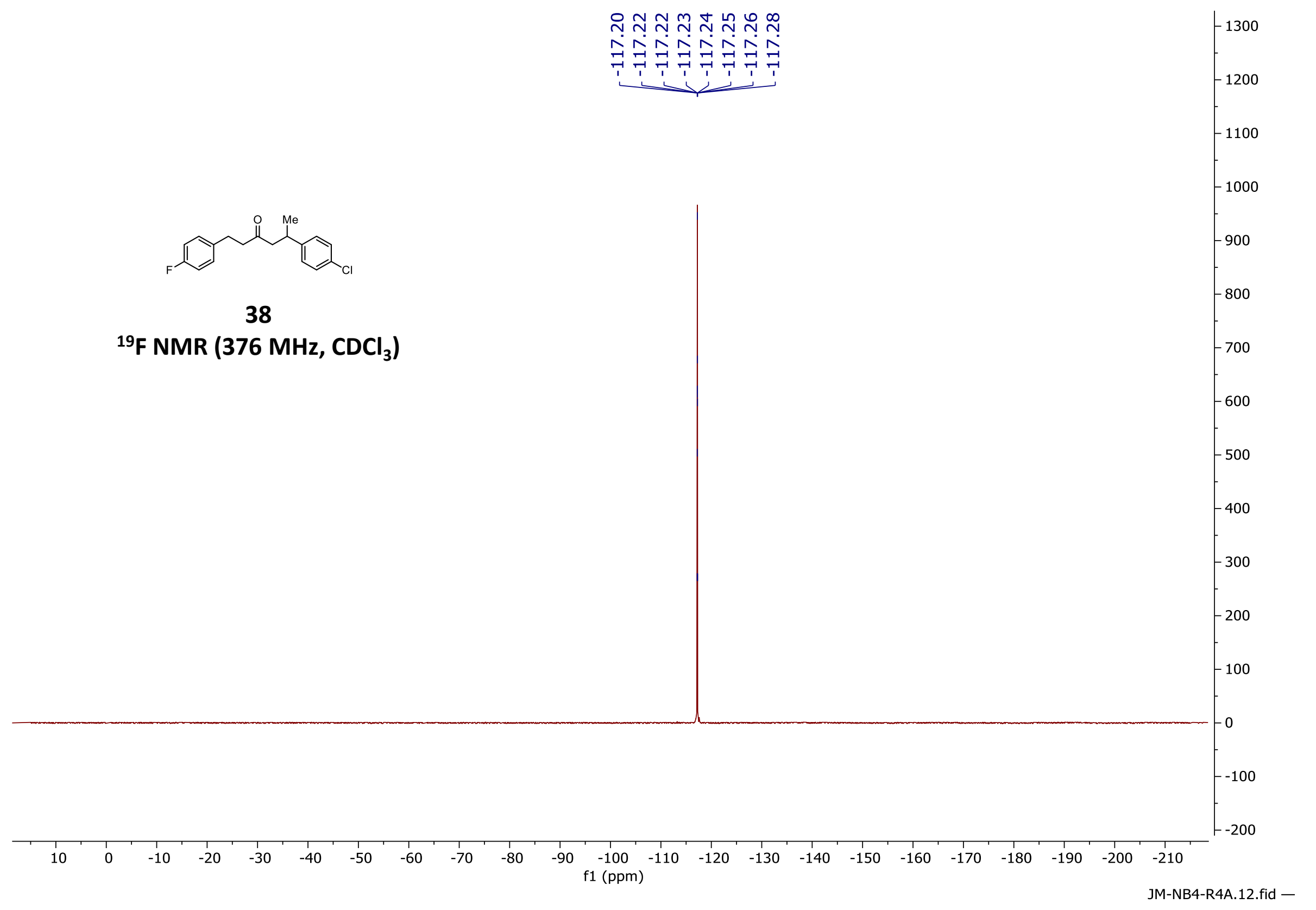




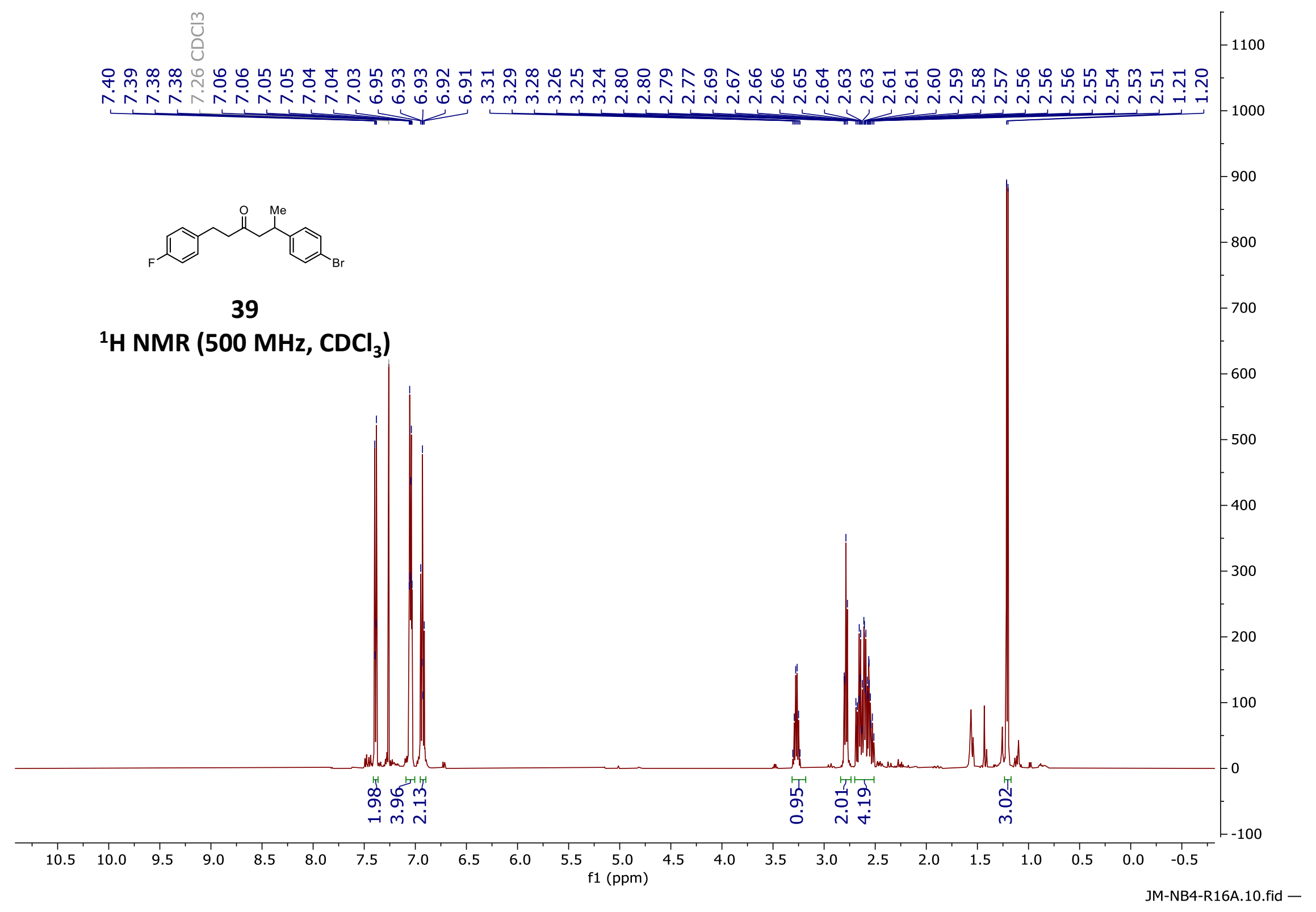




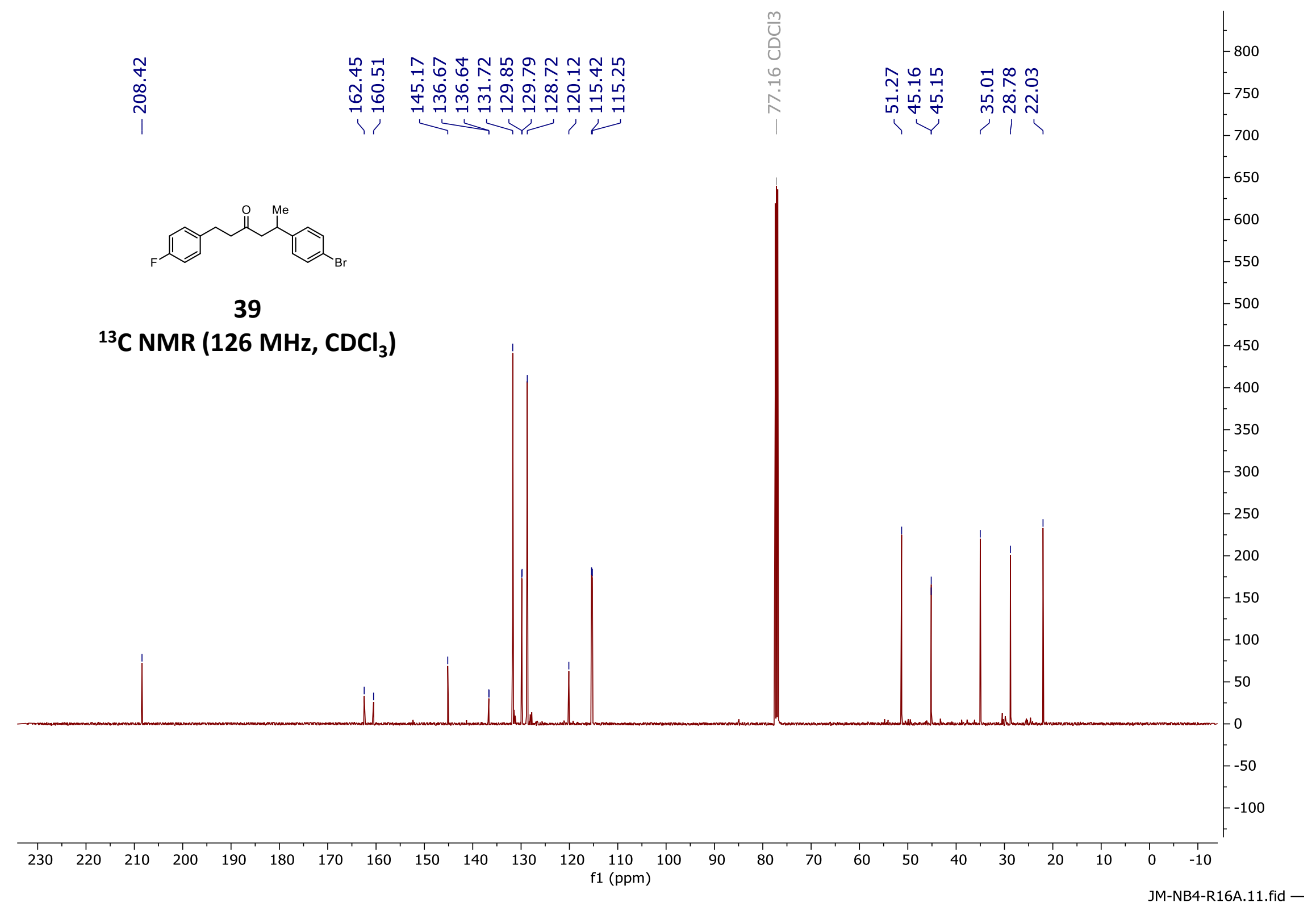




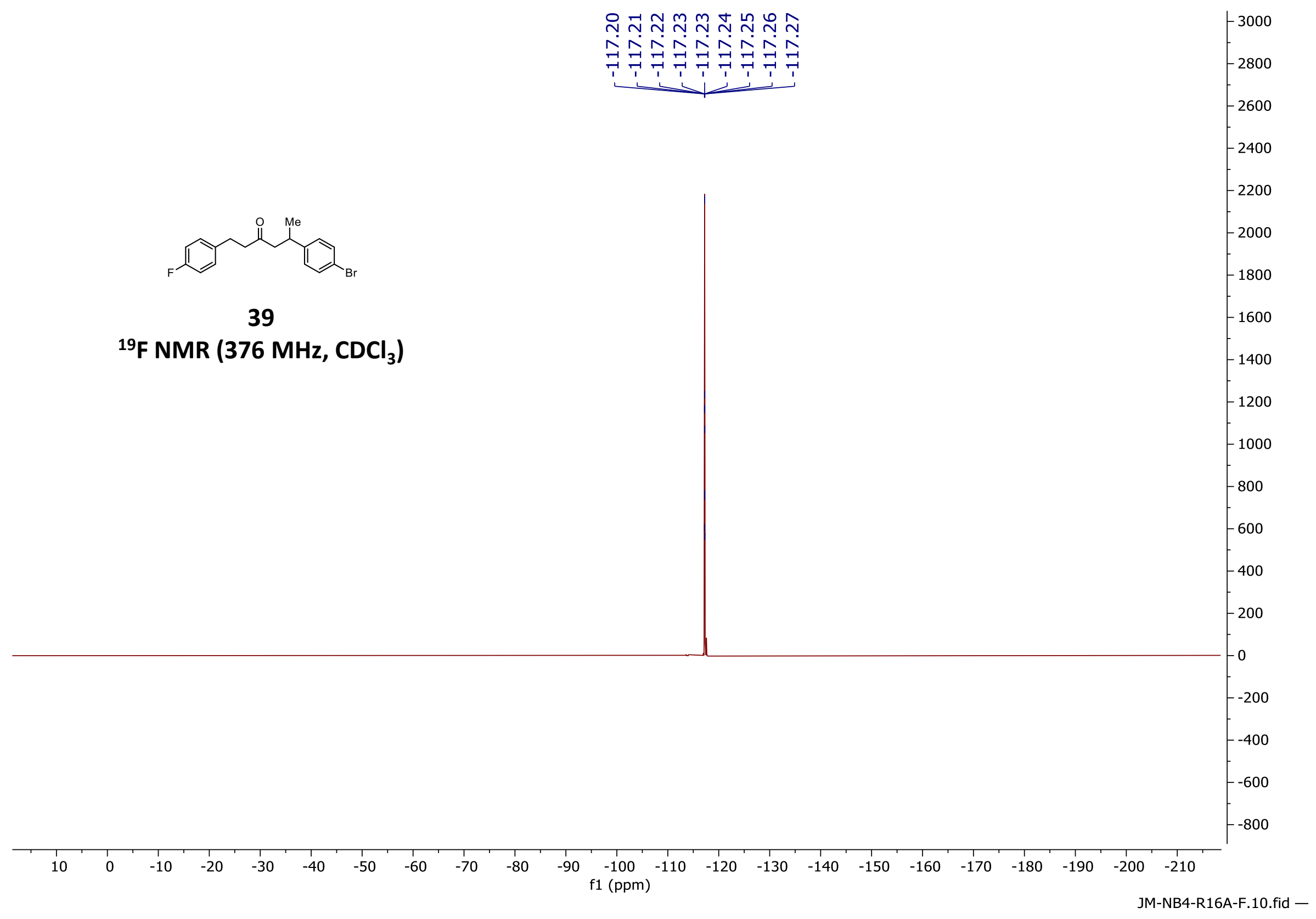




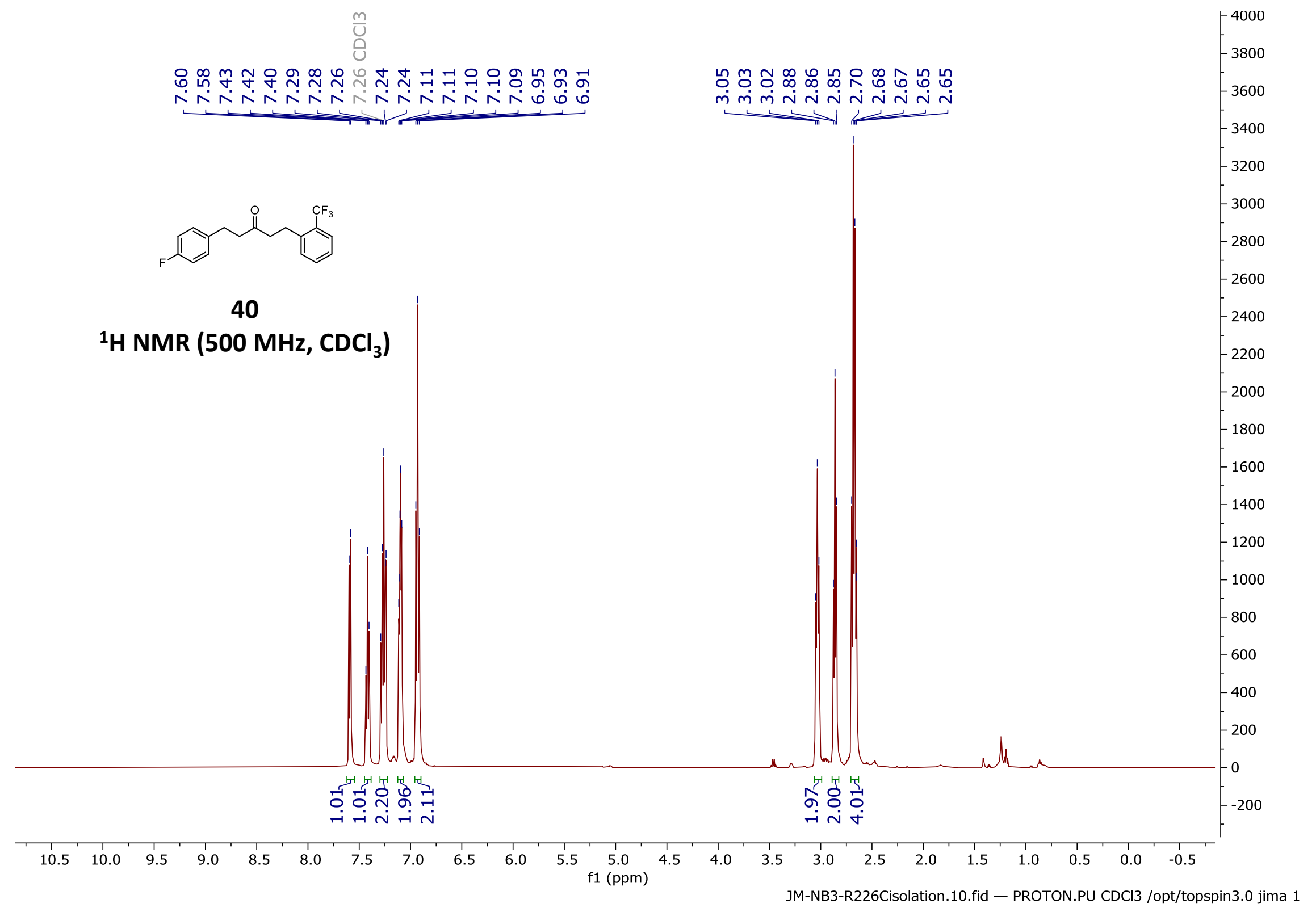




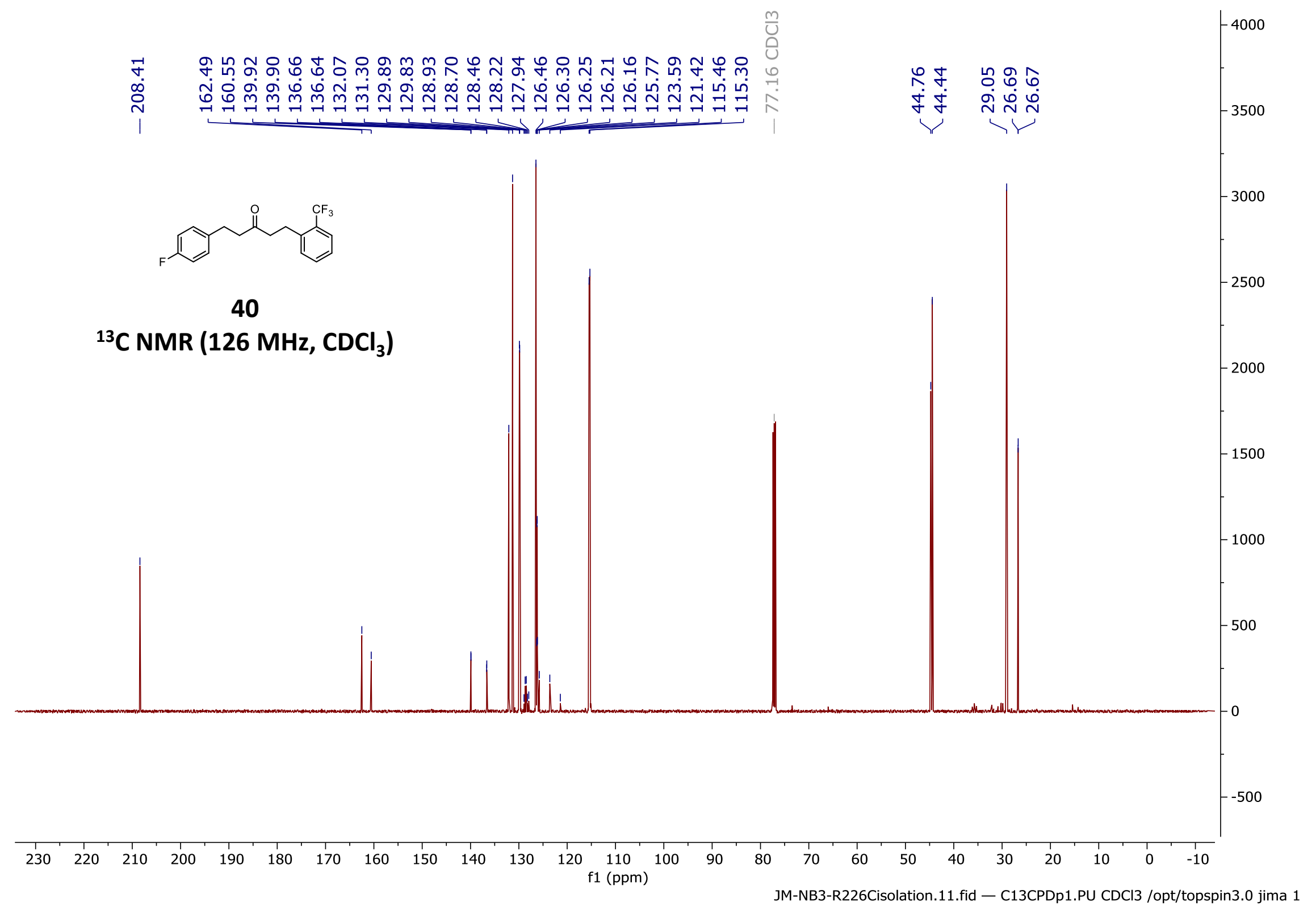




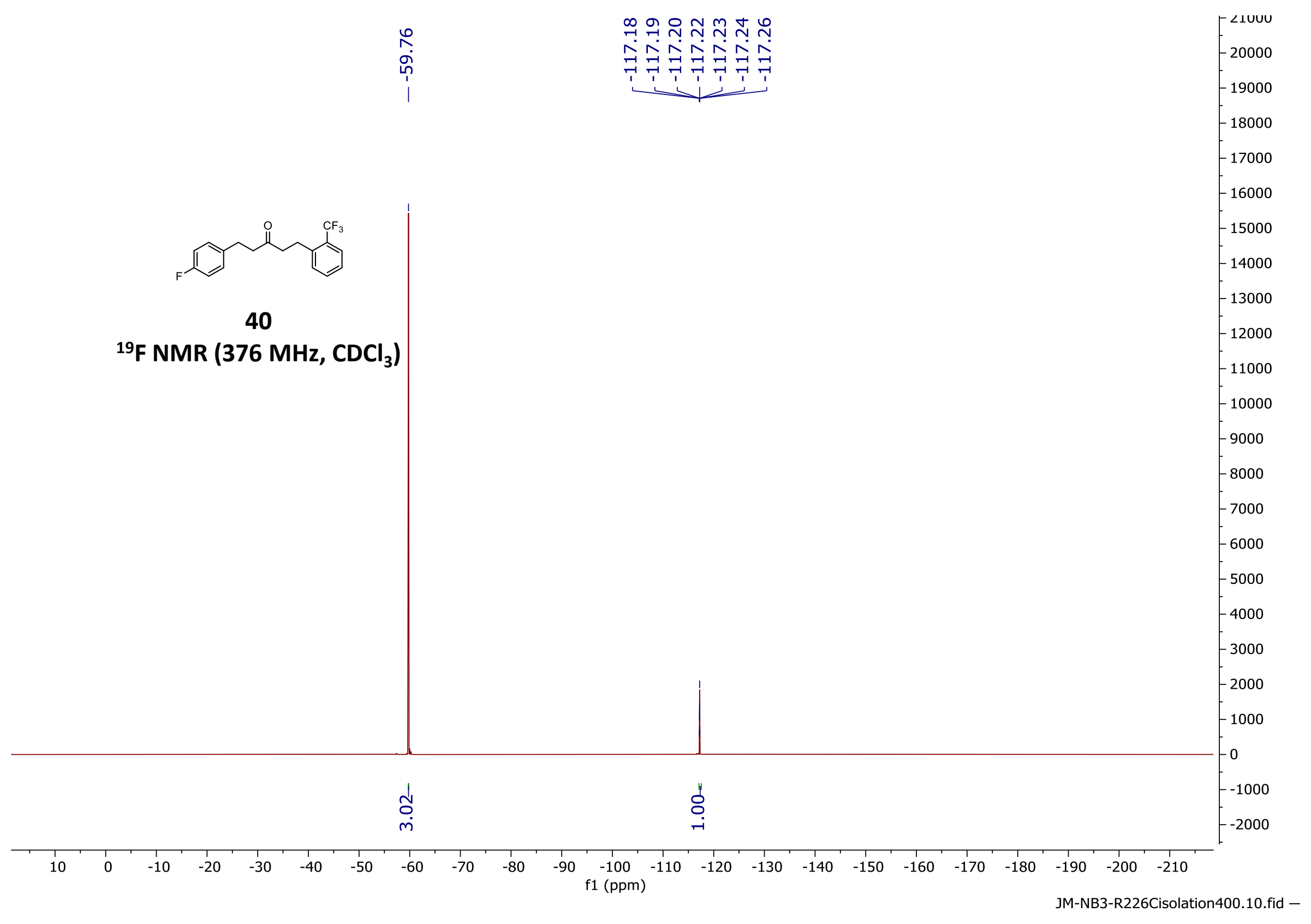




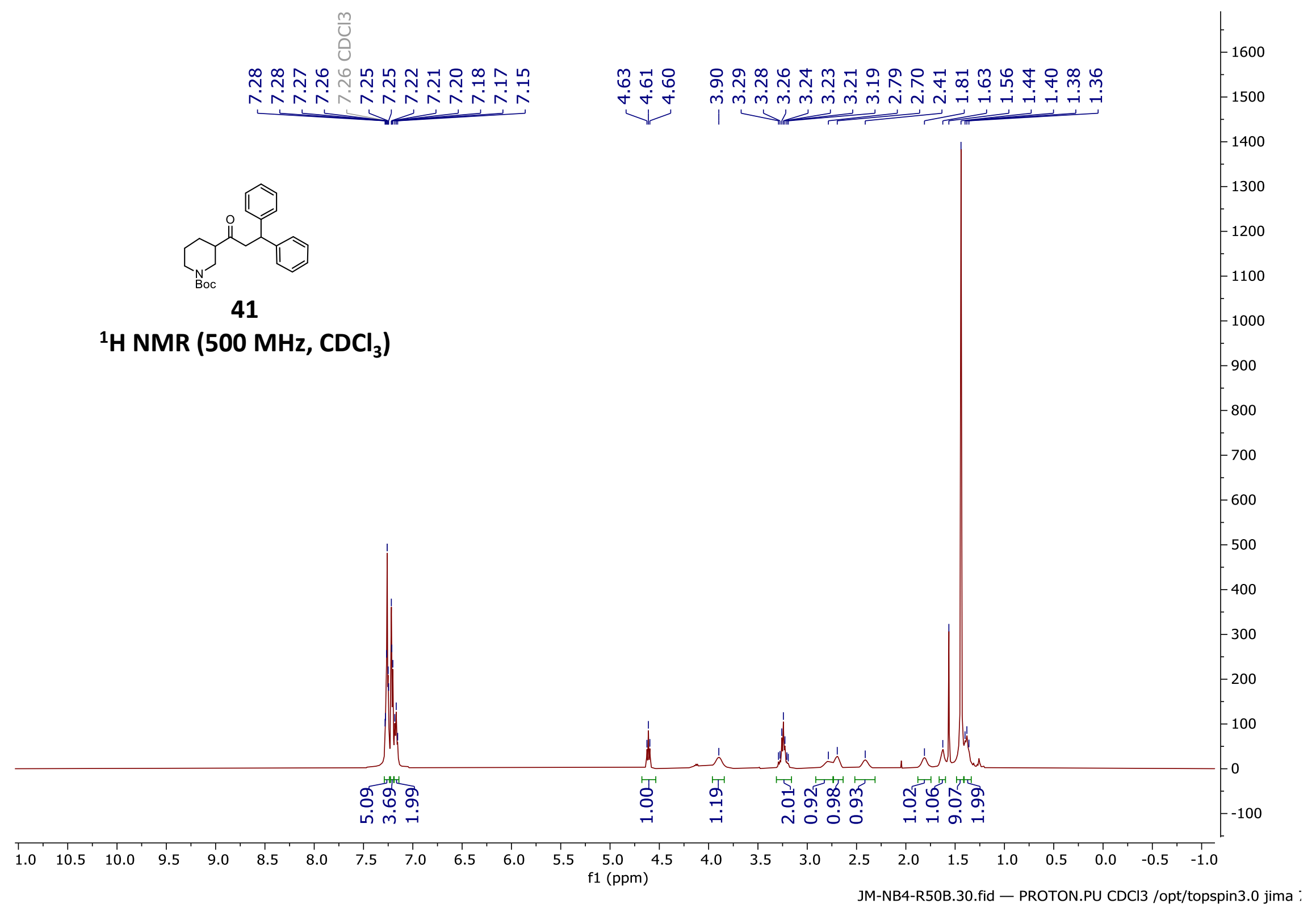




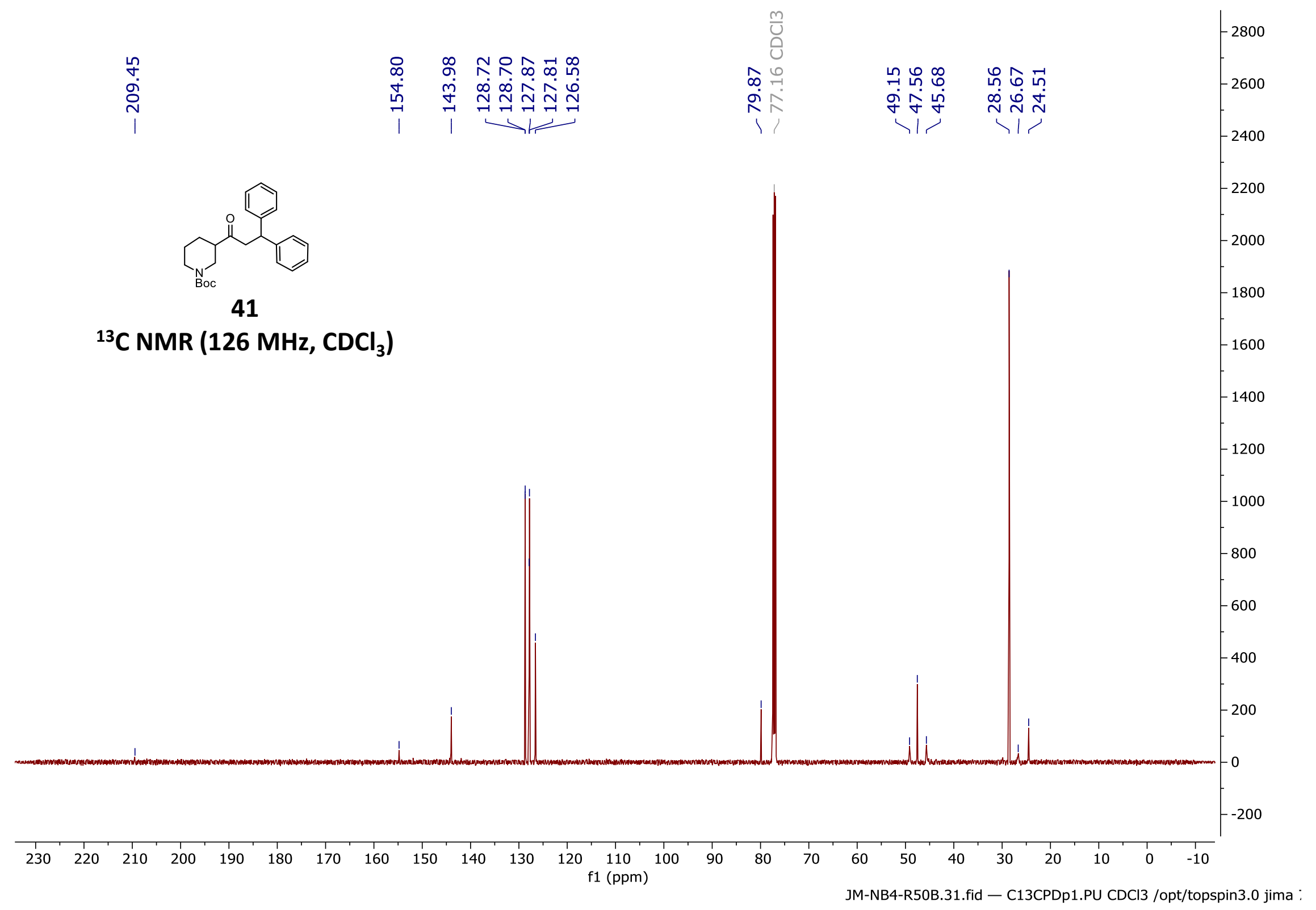

